Florida International University

FIU Digital Commons

$7-2-2020$

\title{
Optimal and Secure Electricity Market Framework for Market Operation of Multi-Microgrid Systems
}

\author{
Mohammad Mahmoudian Esfahani \\ Florida International University, mmahm010@fiu.edu
}

Follow this and additional works at: https://digitalcommons.fiu.edu/etd

Part of the Power and Energy Commons

\section{Recommended Citation}

Mahmoudian Esfahani, Mohammad, "Optimal and Secure Electricity Market Framework for Market Operation of Multi-Microgrid Systems" (2020). FIU Electronic Theses and Dissertations. 4467.

https://digitalcommons.fiu.edu/etd/4467

This work is brought to you for free and open access by the University Graduate School at FIU Digital Commons. It has been accepted for inclusion in FIU Electronic Theses and Dissertations by an authorized administrator of FIU Digital Commons. For more information, please contact dcc@fiu.edu. 


\section{FLORIDA INTERNATIONAL UNIVERSITY \\ Miami, Florida}

\section{OPTIMAL AND SECURE ELECTRICITY MARKET FRAMEWORK FOR MARKET OPERATION OF MULTI-MICROGRID SYSTEMS}

A dissertation submitted in partial fulfillment of

the requirements for the degree of

DOCTOR OF PHILOSOPHY

in

ELECETRICAL AND COMPUTER ENGINEERING

by

Mohammad Mahmoudian Esfahani 
To: Dean John L. Volakis

College of Engineering and Computing

This dissertation, written by Mohammad Mahmoudian Esfahani, and entitled Optimal and Secure Electricity Market Framework for Market Operation of Multi-Microgrid Systems, having been approved in respect to style and intellectual content, is referred to you for judgment.

We have read this dissertation and recommend that it be approved.

Bogdan Carbunar

$\begin{array}{r}\hline \text { Ahmed Ibrahim } \\ \hline \text { Sakhrat Khizroev }\end{array}$

Osama A. Mohammed, Major Professor

Date of Defense: July 2, 2020

The dissertation of Mohammad Mahmoudian Esfahani is approved.

Dean John L. Volakis

College of Engineering and Computing

Andrés G. Gil

Vice President for Research and Economic Development and Dean of the University Graduate School

Florida International University, 2020 
(C) Copyright 2020 by Mohammad Mahmoudian Esfahani

All rights reserved. 


\section{DEDICATION}

To the soul of my lovely sister, Maryam, who left us very soon, but will be in my heart forever.

To my father, Naser, for his everlasting love and support, my family, my love, Mana, and those who without their support and encouragement, the completion of this work would never have been possible. 


\section{ACKNOWLEDGMENTS}

I would like to express my sincere gratitude to my principal advisor, Professor Osama A. Mohammed, for his guidance, support, encouragement, and thought-provoking discussions during my doctoral research. This dissertation would not have been possible without his help, support, resolute dedication, and patience. His passion for success has inspired me to come up with new ideas. His expert advice and unsurpassed knowledge of the various fields of electrical energy systems has always provided me an endless supply of idea to move to the next step and complete this dissertation. I am also grateful for the chance he gave me to work under his supervision at his excellent research group at the Energy Systems Research Laboratory. I highly acknowledge the support I received as a research assistant under my major professor research grants received from the Office of Naval Research and the US Department of Energy throughout my research at the energy systems research laboratory. I thank the university graduate school for awarding me the Dissertation Year Fellowship (DYF) which helped me complete this dissertation.

I would like to also thank my dissertation committee members for their insightful comments and constructive suggestions in the review of my dissertation.

Also, I am thankful to all my colleagues in the Energy Systems Research Laboratory. Many graduate and undergraduate student scholars have helped me with discussions, contributions, and assistance contributing to achieving my research goals.

Finally, I would like to give a special thanks to my father and my family for their unconditional support and love. I also extend my gratitude to for their encouragement and assistance. 


\section{ABSTRACT OF THE DISSERTATION \\ OPTIMAL AND SECURE ELECTRICITY MARKET FRAMEWORK FOR MARKET OPERATION OF MULTI-MICROGRID SYSTEMS}

by

Mohammad Mahmoudian Esfahani

Florida International University, 2020

Miami, Florida

\section{Professor Osama A. Mohammed, Major Professor}

Traditional power systems were typically based on bulk energy services by large utility companies. However, microgrids and distributed generations have changed the structure of modern power systems as well as electricity markets. Therefore, restructured electricity markets are needed to address energy transactions in modern power systems.

In this dissertation, we developed a hierarchical and decentralized electricity market framework for multi-microgrid systems, which clears energy transactions through three market levels; Day-Ahead-Market (DAM), Hour-Ahead-Market (HAM) and Real-TimeMarket (RTM). In this market, energy trades are possible between all participants within the microgrids as well as inter-microgrids transactions. In this approach, we developed a game-theoretic-based double auction mechanism for energy transactions in the DAM, while HAM and RTM are cleared by an optimization algorithm and reverse action mechanism, respectively. For data exchange among market players, we developed a secure data-centric communication approach using the Data Distribution Service. Results demonstrated that this electricity market could significantly reduce the energy price and dependency of the multi-microgrid area on the external grid. 
Furthermore, we developed and verified a hierarchical blockchain-based energy transaction framework for a multi-microgrid system. This framework has a unique structure, which makes it possible to check the feasibility of energy transactions from the power system point of view by evaluating transmission system constraints. The blockchain ledger summarization, microgrid equivalent model development, and market players' security and privacy enhancement are new approaches to this framework.

The research in this dissertation also addresses some ancillary services in power markets such as an optimal power routing in unbalanced microgrids, where we developed a multi-objective optimization model and verified its ability to minimize the power imbalance factor, active power losses and voltage deviation in an unbalanced microgrid.

Moreover, we developed an adaptive real-time congestion management algorithm to mitigate congestions in transmission systems using dynamic thermal ratings of transmission lines. Results indicated that the developed algorithm is cost-effective, fast, and reliable for real-time congestion management cases.

Finally, we completed research about the communication framework and security algorithm for IEC 61850 Routable GOOSE messages and developed an advanced protection scheme as its application in modern power systems. 
Chapter 1 Introduction and Research Background................................................. 1

$1.1 \quad$ An introduction to electricity markets ...................................................... 3

1.1.1 Electricity market types .................................................................... 3

1.1.2 Trading methods in electricity markets....................................................... 4

1.1.3 Wholesale and retail electricity markets ………………………................ 5

1.1.4 Benefits from power market deregulations ............................................... 6

$1.2 \quad$ Challenges in electricity market deregulations........................................... 7

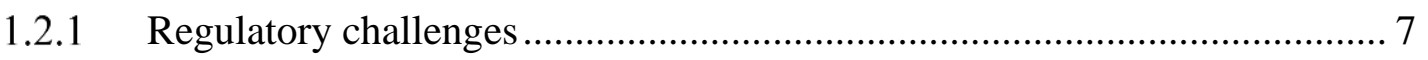

1.2.2 Modern power system challenges ........................................................ 8

1.2.3 Communication challenges .................................................................. 8

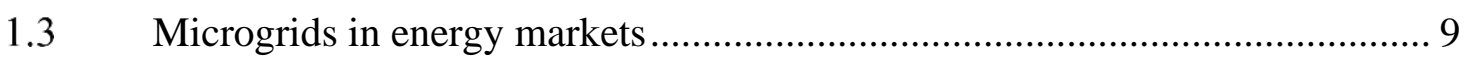

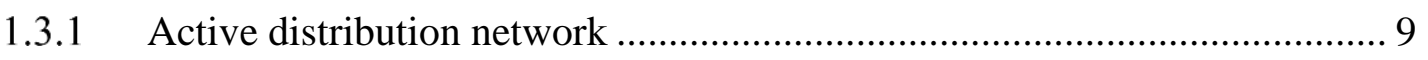

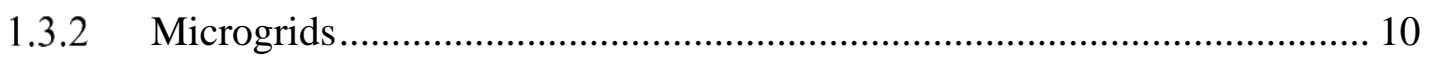

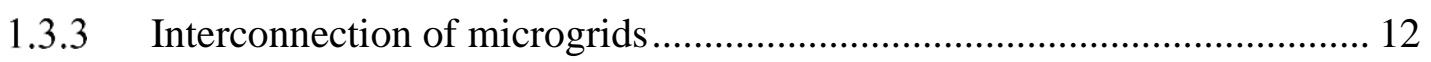

1.3.4 Microgrid power markets................................................................. 12

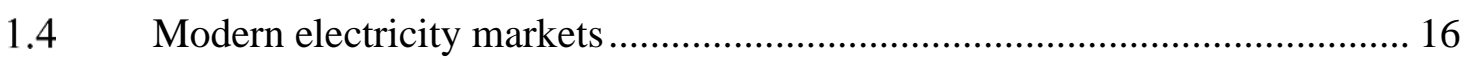

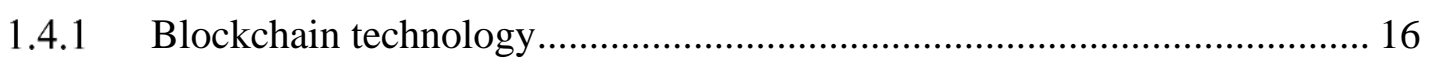

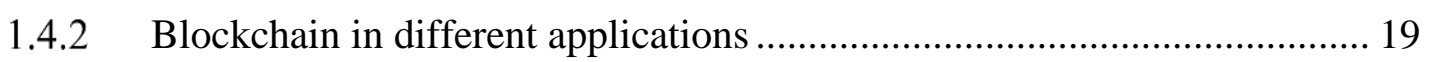

1.4.3 Blockchain in electricity markets.......................................................... 23

1.4.4 Challenges of using Blockchain in power markets..................................... 25

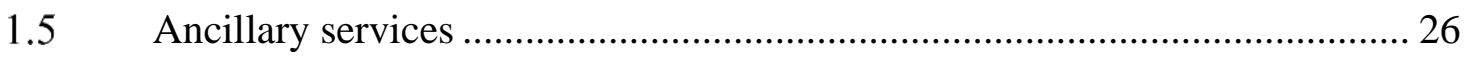

1.5.1 Real-Time Congestion Management (RTCM) …….................................. 26

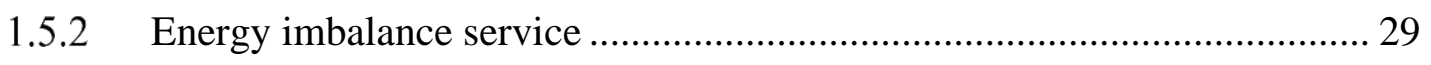

1.6 Critical communications in modern power systems ....................................... 32

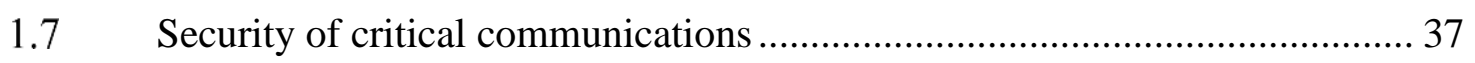

$1.8 \quad$ Advanced protection in modern power systems .......................................... 38

1.8.1 High fault currents in power systems.................................................... 38

1.8.2 Communication capabilities in the power system protection ..................... 40

1.9 Problem statements ................................................................................... 41

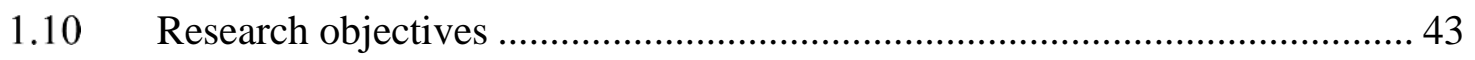

1.11 Original contributions of this dissertation .................................................... 45 


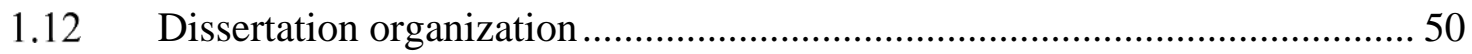

Chapter 2 Microgrids' Participation in Power Markets.................................................. 54

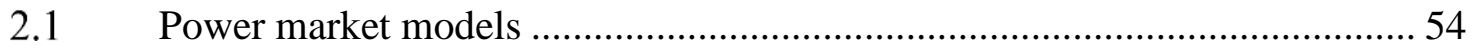

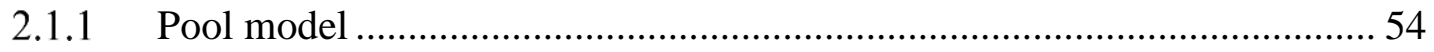

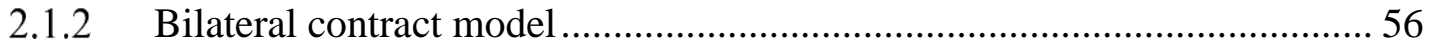

2.1.3 Hybrid model ................................................................................... 56

2.2 Microgrids in competitive power markets .................................................. 57

2.2.1 Participation in the retail market............................................................. 57

2.2.2 Participation in ancillary services ........................................................... 58

2.3 Multi-Microgrid Power Markets .............................................................. 58

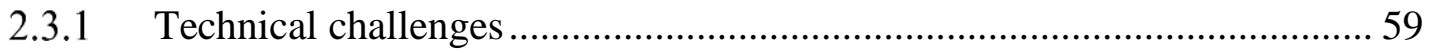

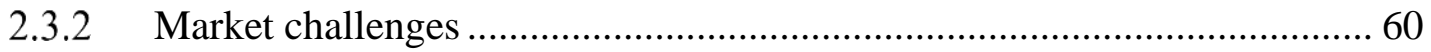

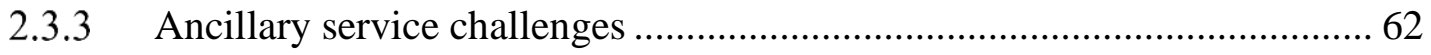

Chapter 3 Electricity Market Security Assessment ..................................................... 64

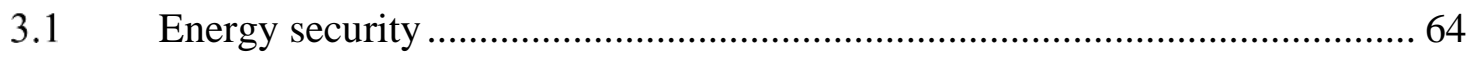

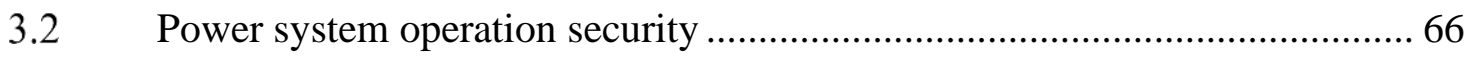

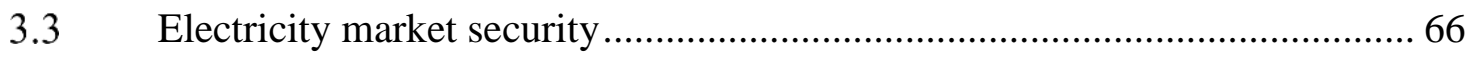

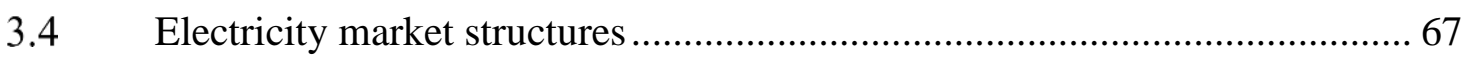

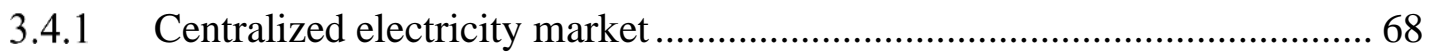

3.4.2 Decentralized electricity market ………………...................................... 69

3.4.3 Distributed electricity market ……………………………………….... 70

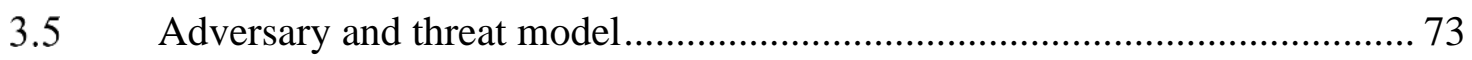

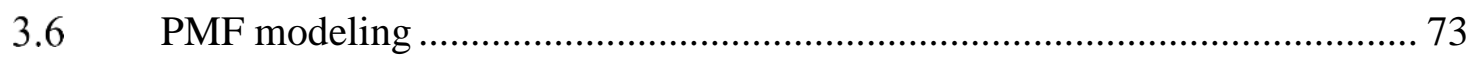

3.6.1 PMF model for centralized marketplace ………….................................. 74

3.6.2 PMF model for decentralized marketplace .............................................. 74

3.6.3 PMF model for distributed marketplace ………………………….......... 75

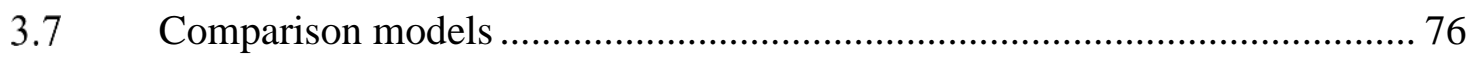

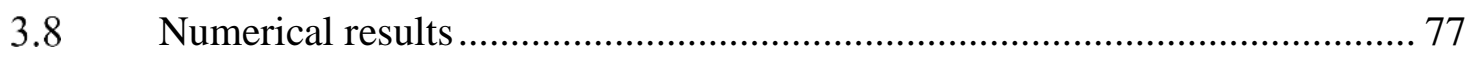

Chapter 4 Optimal Decentralized Electricity Market for Multi-Microgrid Systems... 79

4.1 Architecture of the multi-microgrid system ……........................................ 80

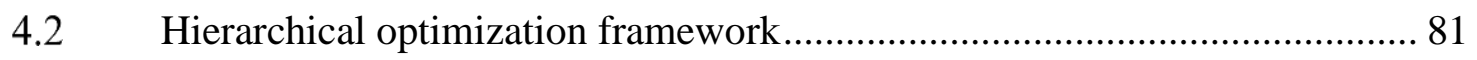


4.3 Developed energy market framework ………........................................... 83

4.3.1 Level 1 - Day-Ahead Market …………………………………………..... 84

4.3.2 Level 2 - Hour-Ahead Market................................................................ 90

4.3.3 Level 3 - Real-Time Market ............................................................. 92

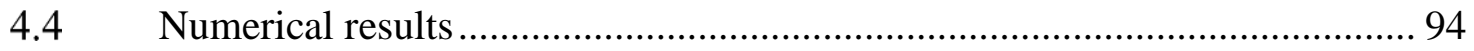

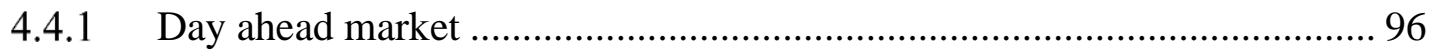

4.4.2 Hour ahead market ............................................................................. 98

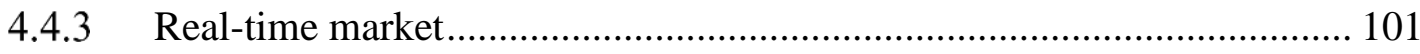

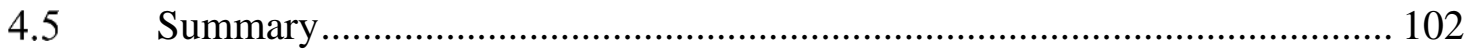

Chapter 5 Secure Communication Platform for Decentralized Electricity Market... 104

5.1 Message-Centric vs. Data-Centric communication approaches .................... 105

5.1.1 Message centric communication approach ............................................. 106

5.1.2 Data centric communication approach..................................................... 106

5.2 Data Distribution Service (DDS) ........................................................... 107

5.3 Proposed Communication Platform........................................................... 108

5.4 Development of the proposed communication platform ............................. 110

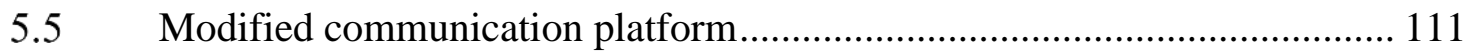

5.6 Security of DDS Framework …………............................................ 113

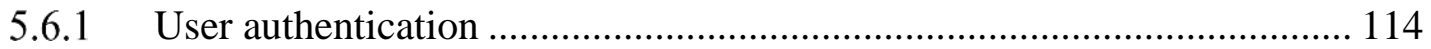

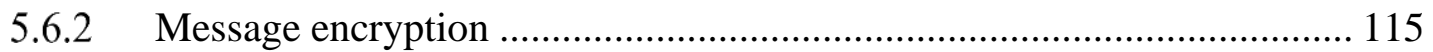

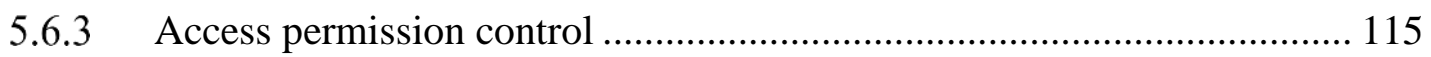

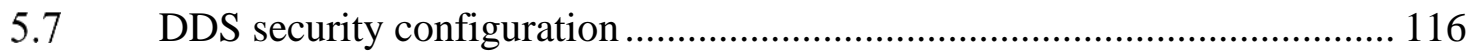

5.8 Security of the Modified Communication Platform ..................................... 117

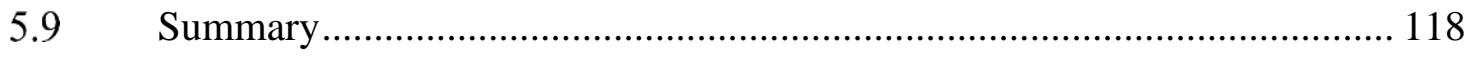

Chapter 6 Hierarchical Blockchain-Based Energy Transaction Framework............. 120

6.1 Electricity market models; ros and Cons ................................................ 120

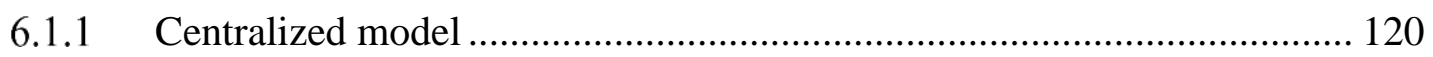

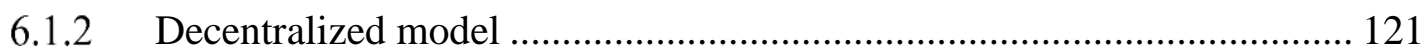

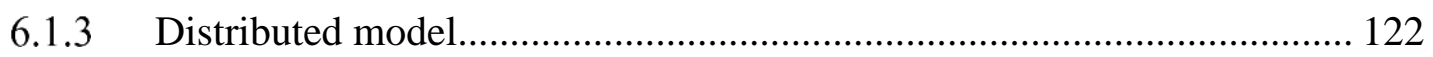

6.2 Hierarchical Blockchain-based Market Model........................................... 125

6.2.1 The proposed HBC model for multi-microgrid systems ........................... 126

6.2.2 Model description ................................................................................ 127 
6.2.3 HBC model development.................................................................. 129

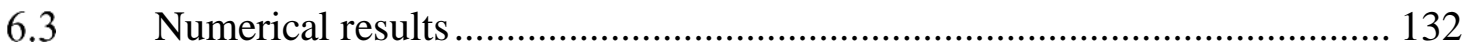

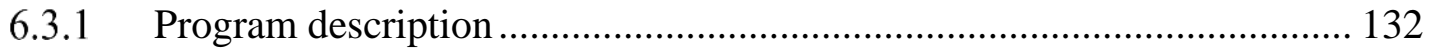

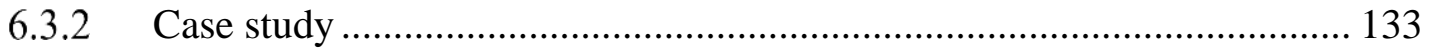

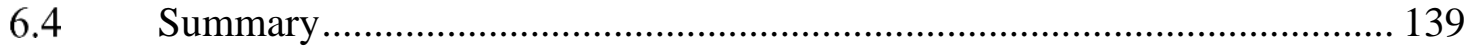

Chapter 7 Optimal Operation of Unbalanced Hybrid AC-DC Microgrids ................ 140

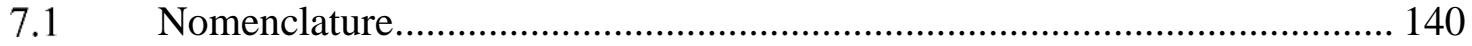

7.2 Control models of Interlinking Converters .................................................. 142

7.3 The developed OPR scheme.................................................................. 143

7.3.1 Multi-objective optimization model ....................................................... 144

7.3.2 Particle Swarm Optimization (PSO) …………….................................. 148

7.3.3 Microgrid operating index .................................................................. 149

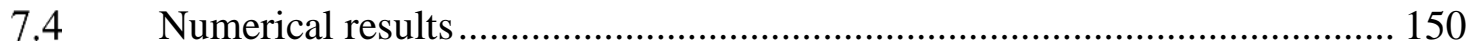

7.4.1 Modified IEEE 13 bus system …………………………………......... 151

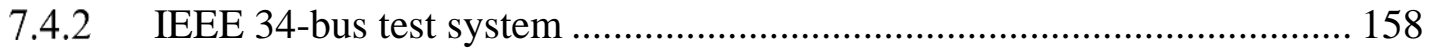

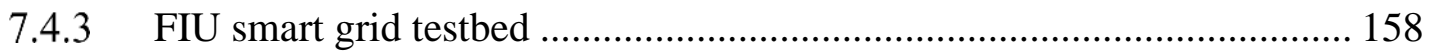

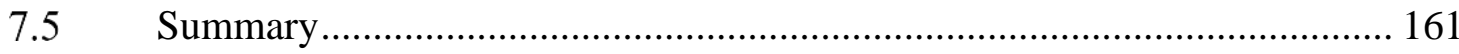

Chapter 8 Real-Time Congestion Management in Power Markets ............................ 163

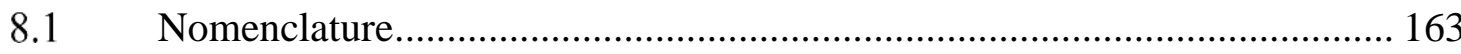

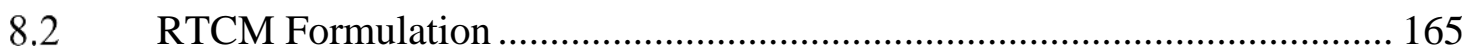

8.3 Short and Emergency Thermal Ratings.................................................... 167

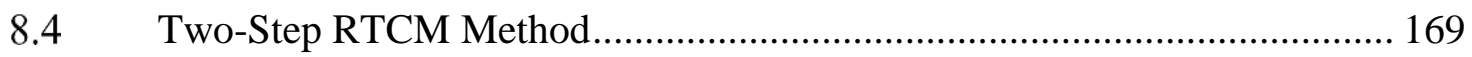

8.5 Real-Time hybrid optimization algorithm............................................. 171

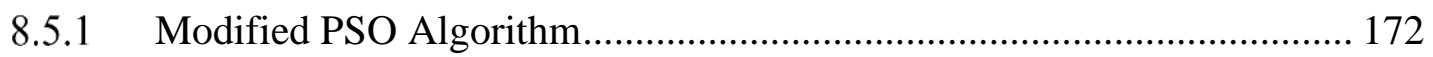

8.5.2 Adaptive Artificial Neural Network ………………............................ 174

8.6 The developed adaptive RTCM Algorithm .............................................. 177

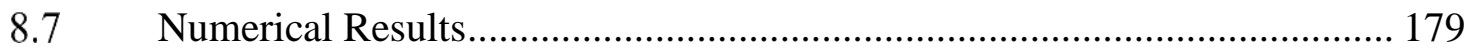

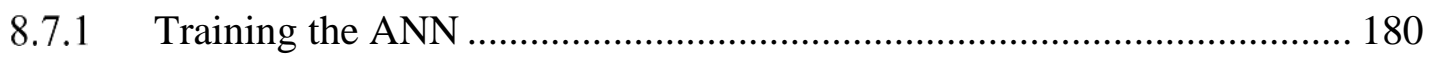

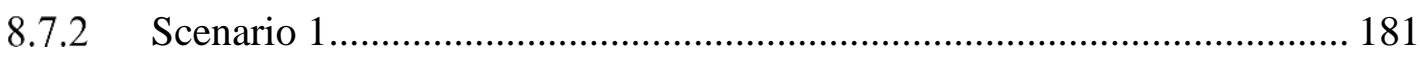

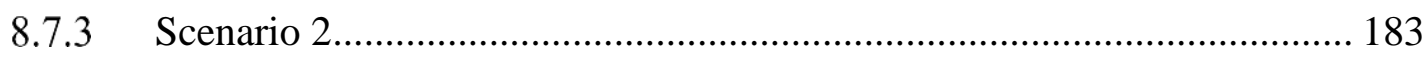

$8.8 \quad$ Summary 
Chapter 9 Critical Communications in Modern Power Systems ................................ 186

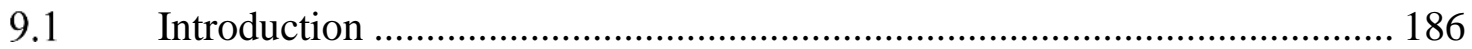

9.2 The Proposed MRGM Framework …………………………………......... 187

9.2.1 Conversion: GOOSE Data Model to DDS Data Object ............................ 189

9.2.2 Message Encapsulating and Routing Service ............................................ 190

9.2.3 Conversion: DDS Data Object to GOOSE Data Model ............................ 191

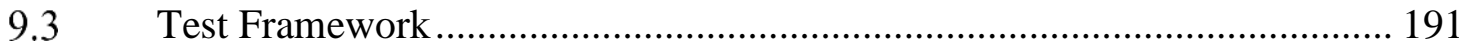

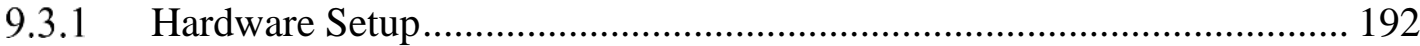

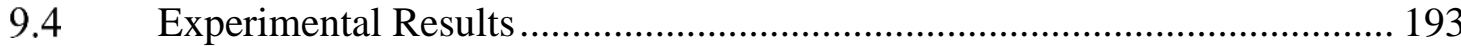

9.4.1 GOOSE Publish-Subscribe over Layer Two: Case 1 ................................. 193

9.4.2 DDS Publish-Subscribe over the Network Layer: Case2 ……………...... 195

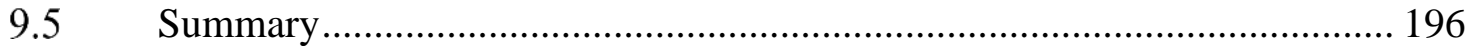

Chapter 10 Security of Critical Communications in Power Systems ............................ 197

10.1 DDS Security Mechanism for the MRGM Framework................................... 198

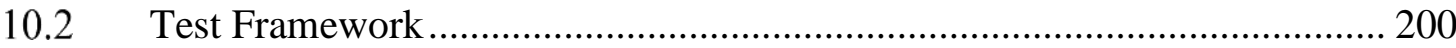

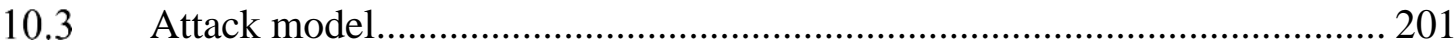

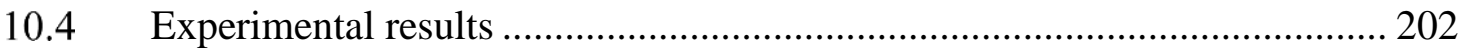

10.4.1 The latency of the security algorithm .................................................... 202

10.4.2 Cyber-attack on the DDS-Based MRGM framework................................ 203

10.5 Secure Sequence Hopping Algorithm for Routable Communications.......... 205

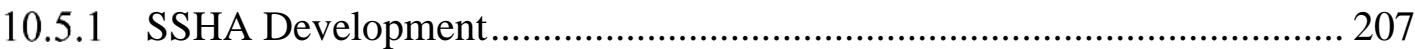

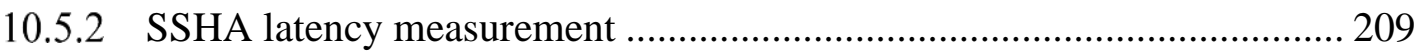

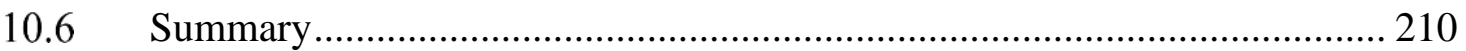

Chapter 11 Application of Critical Communications in Modern Power System ........ 211

11.1 Dealing with fault current in power systems ............................................... 211

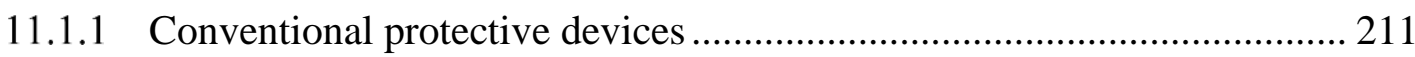

11.1.2 Limiting the high level fault current ....................................................... 213

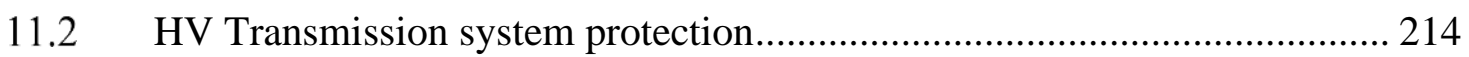

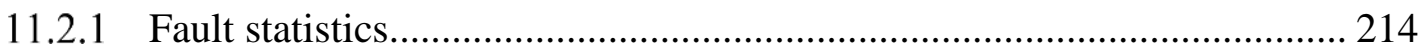

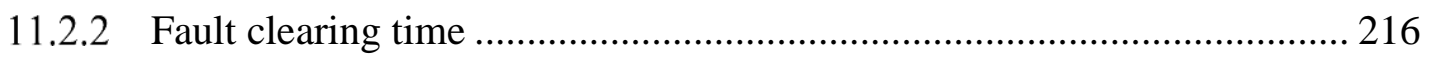

11.3 The developed High Current Protection Scheme ……………………....... 218

$11.4 \quad$ Flowchart of the developed HCPS …………........................................... 220 
11.5 Flexibility and reliability of the developed HCPS........................................ 224

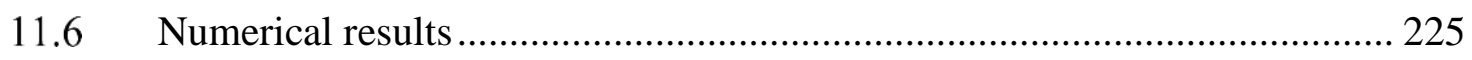

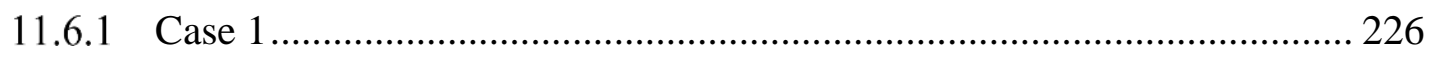

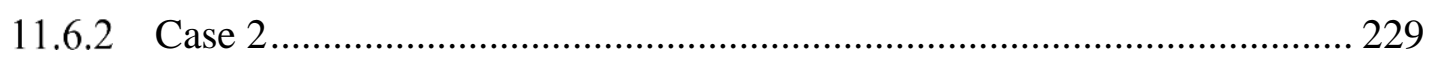

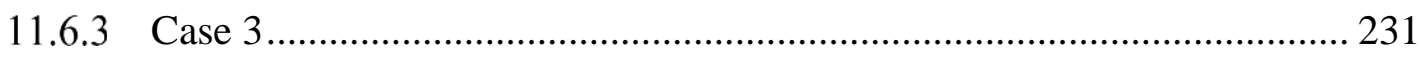

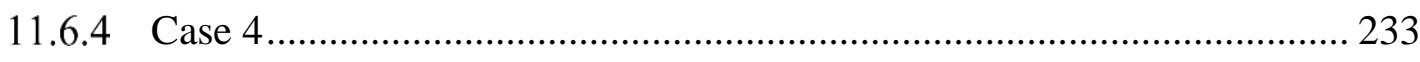

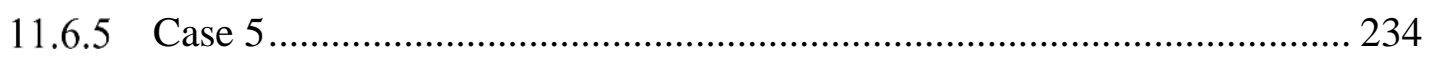

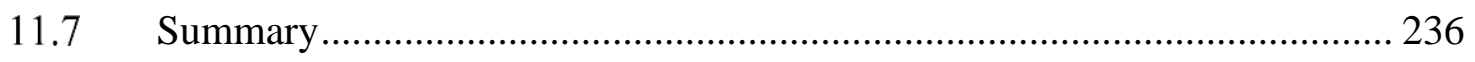

Chapter 12 Conclusions and Recommendations for Future Work ………………..... 238

$12.1 \quad$ Recommendations for Future Work ……………..................................... 243

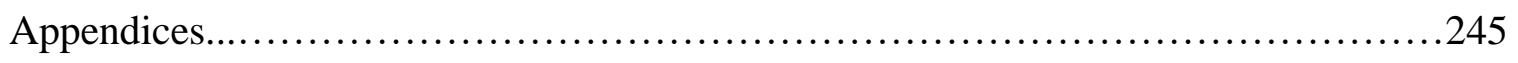

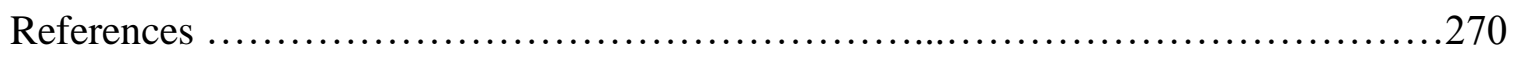

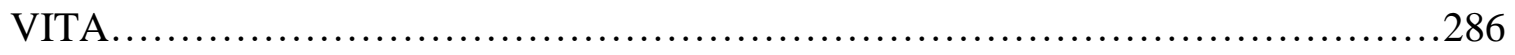




\section{LIST OF TABLES}

TABLE

PAGE

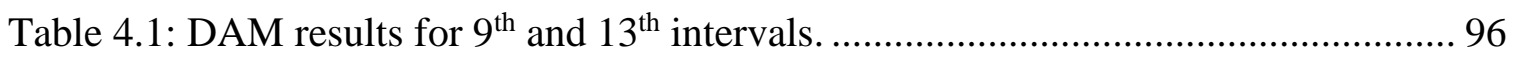

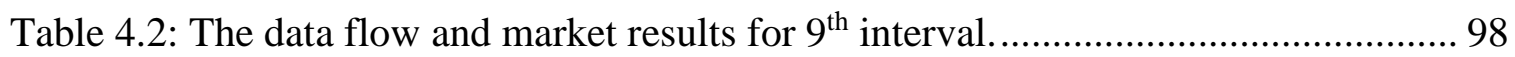

Table 6.1: Comparison of energy transaction frameworks for the centralized, dcentralized, distributed and hybrid market models............................................... 124

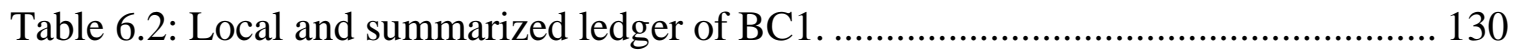

Table 6.3: Transaction requests for inter-MG market. .......................................... 136

Table 6.4: Final ledger for inter-MG blockchain................................................. 136

Table 6.5: Additional blocks to update the local BCs ledgers.................................... 138

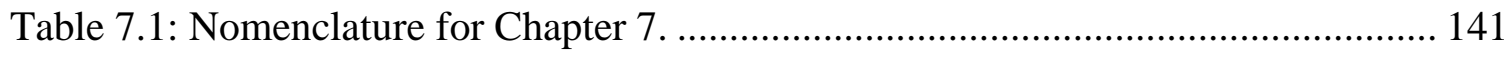

Table 7.2: Power routing mechanism and objective functions for case studies. ............ 150

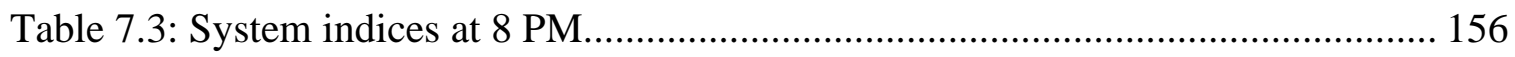

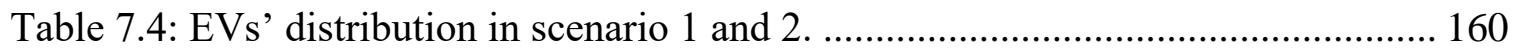

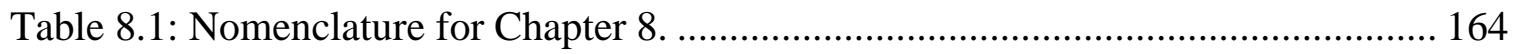

Table 8.2: Generators' data for the modified 39-bus New England system. ................. 180

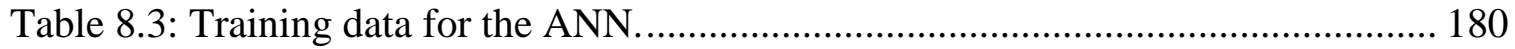

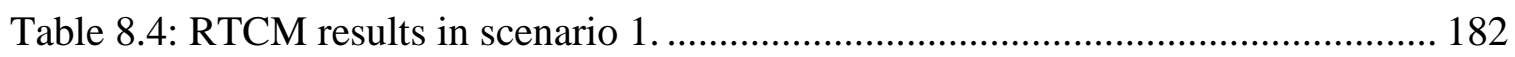

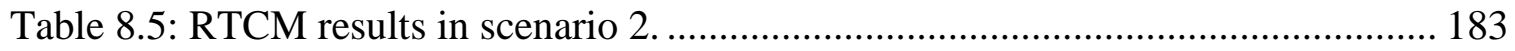

Table 9.1: Measurement results. ............................................................................... 194

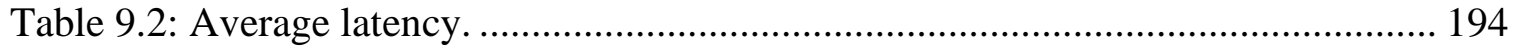

Table 11.1: Breaking capacity of CBs in different voltage levels............................. 212

Table 11.2: Time delay for each section of the protection systems............................ 218

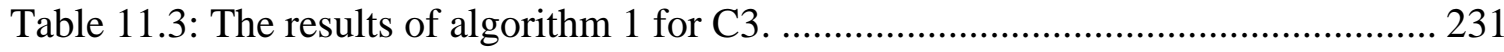




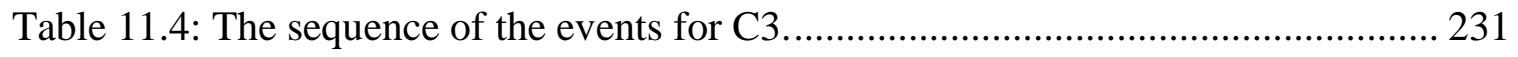

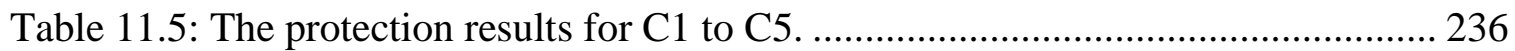




\section{LIST OF FIGURES}

FIGURE

PAGE

Figure 1.1: Monopoly Vs. Competitive Power Market ...................................................... 3

Figure 1.2: Wholesale and retail electricity markets ………………………………...... 5

Figure 1.3: Configuration of a typical MG [16] ..................................................... 11

Figure 1.4: Centralized and distributed transaction models [45] .................................... 17

Figure 1.5: Applications of the $\mathrm{BC}$ technology in different fields [62] .......................... 20

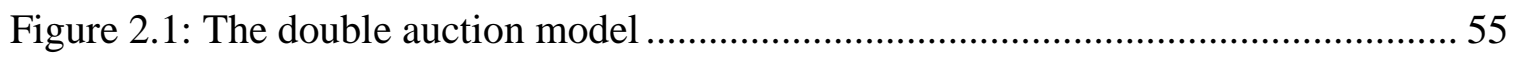

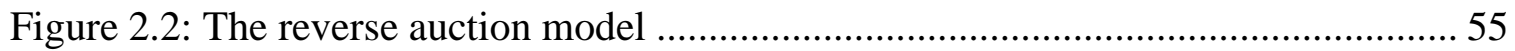

Figure 2.3: The concept of multi-microgrid [143] ................................................... 59

Figure 3.1: Electricity market structures and communication links [158]....................... 68

Figure 3.2: The $\mathrm{BC}$ algorithm to build and process a new block by processing the transactions' validity and the mining process................................................................... 72

Figure 3.3: The PSA for different sections and communication links of three market

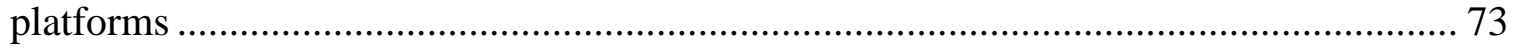

Figure 3.4: PMF of the centralized market ………………....................................... 78

Figure 3.5: PMF of the decentralized market ……………...................................... 78

Figure 3.6: PMF of the distributed market.................................................................. 78

Figure 3.7: PMF of the three market frameworks.................................................... 78

Figure 4.1: Architecture of a Multiagent-based multi-microgrid system. ...................... 80

Figure 4.2: Hierarchical optimization framework for a multi-microgrid system with leaders and follower for three MGs, LMAs, Utility and GMA. ...................................... 81

Figure 4.3: Proposed algorithm for the multi-level market operation including DAM,

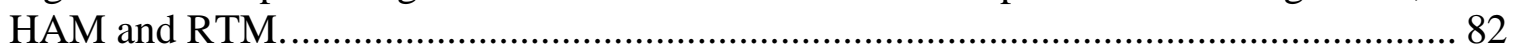

Figure 4.4: Data exchange flowchart between market agents for the developed market framework. 
Figure 4.5: Double auction mechanism

Figure 4.6: LA's bidding strategy for the DAM.

Figure 4.7: Generator's cost function and price bids calculations for participation in the double auction.

Figure 4.8: Reverse auction mechanism indicating the competition between energy providers in the RTM.

Figure 4.9: The modified IEEE 37-bus distributed network including three MGs in grid-connected mode.

Figure 4.10: Interface between agents developed in DigSilent PowerFactory and DDS through DDE.

Figure 4.11: The Game theory based DAM results for MG1 at $9^{\text {th }}$ and $13^{\text {th }}$ market intervals.

Figure 4.12: load and generation profiles 99

Figure 4.13: DAM, HAM and RTM result 99

Figure 4.14: Reverse auction result for $9^{\text {th }}$ RTM interval. 101

Figure 5.1: Developed communication platform for MAS based hierarchical market structure.

Figure 5.2: Connectivity Visualization of the DDS including Participants and DDS topics within a DDS domain.

Figure 5.3: The modified communication platform with reduced topics for each MG. . 112

Figure 5.4: DDS topics with ID management to optimize the communication framework and reduce the number of topics within a DDS domain.

Figure 5.5: Security configurations for the modified communication platform for the MAS-based decentralized electricity market for Multi-Microgrid systems.

Figure 6.1: Hierarchical blockchain model developed by S. Sahoo et al. [181]. 125

Figure 6.2: Conventional blockchain-based energy transaction framework with all agents in a single $\mathrm{BC}$ 
Figure 6.3: The proposed HBC energy transaction framework for a Multi-MG system including two layers for energy transactions.

Figure 6.4: Energy cost functions in equivalent model of MG1 in the HBC model. ..... 131

Figure 6.5: Area's equivalent grid model for inter-microgrid transactions .................... 131

Figure 6.6: Transaction structure and verification process in the BC technology.......... 132

Figure 6.7: Power grid and equivalent model for BC-based transactions. ..................... 134

Figure 6.8: Summarized ledgers for agents in the Inter-MG layer for energy transactions between MGs.

Figure 6.9: Eclipse console view for the HBC results considering the power system constraints before approving each transaction.

Figure 6.10: Eclipse console view for the HBC without considering the power system constraints in transaction approval process............................................................... 138

Figure 7.1: Symmetric (a) and asymmetric (b) control models for Interlinking converters.

Figure 7.2: The proposed optimal power routing scheme for unbalanced hybrid AC-DC MGs in grid-connected mode using PSO algorithm and unbalanced power flow calculations.

Figure 7.3: The modified IEEE 13-bus system as an unbalance hybrid AC-DC microgrid.

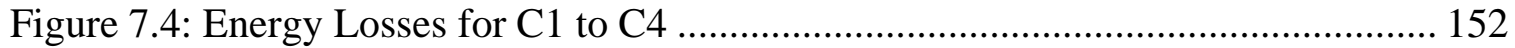

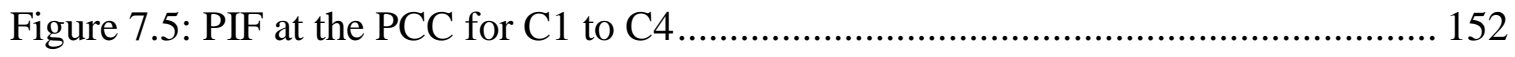

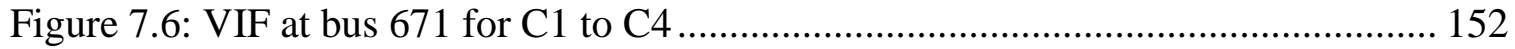

Figure 7.7: Objective function values for each iteration of PSO ................................. 153

Figure 7.8: The values of PIF, VDI and APLI for each iteration of PSO....................... 153

Figure 7.9: Microgrid active power losses and loadability for $\mathrm{C} 1$ to $\mathrm{C} 4 \ldots \ldots \ldots \ldots \ldots \ldots \ldots \ldots \ldots . . . . . . .154$

Figure 7.10: EVs distribution in base and optimum cases.......................................... 154

Figure 7.11: Active power value for each phase of IC\#1 for $\mathrm{C} 1$ to $\mathrm{C} 4$.......................... 154 
Figure 7.12: Dynamic simulation of Microgrid losses for $\mathrm{C} 1 \& \mathrm{C} 4$............................ 155

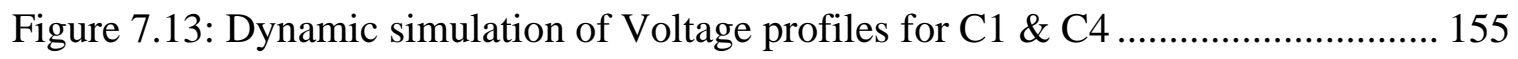

Figure 7.14: Modified IEEE 34-bus test system with six ICs.................................. 157

Figure 7.15: Objective function values for IEEE 34-bus system.............................. 157

Figure 7.16: Reference active power for ICs for base and optimal Scenarios............... 157

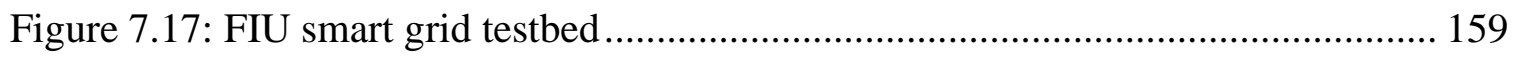

Figure 7.18: Single line diagram of the seven bus test system at FIU ......................... 159

Figure 7.19: Hardware-Software interoperability at FIU smart grid .......................... 159

Figure 7.20: Load distribution in scenario1 …................................................ 160

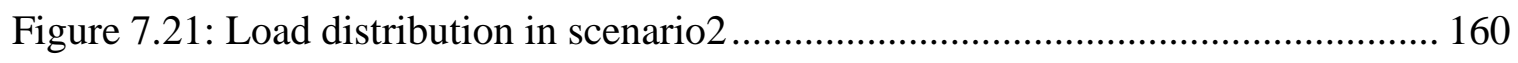

Figure 7.22: Total generation and load in both scenarios ...................................... 160

Figure 7.23: Active power losses in both scenarios............................................... 160

Figure 8.1: Determination of STR and ETR rates [101] ....................................... 168

Figure 8.2: The concept of adaptive RTCM method.......................................... 170

Figure 8.3: Nominal band limit determination in the adaptive RTCM algorithm ......... 173

Figure 8.4: Architecture of the back-propagation model for the AANN...................... 175

Figure 8.5: The developed adaptive RTCM algorithm....................................... 178

Figure 8.6: The modified 39-bus New England system with two PSTs [102].............. 180

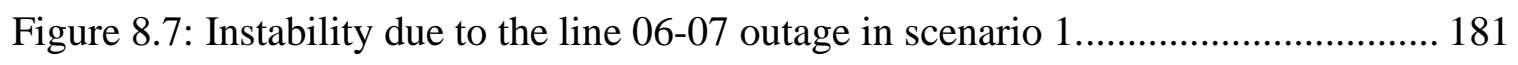

Figure 8.8: Rescheduling values for all solution in scenario 1 ................................. 182

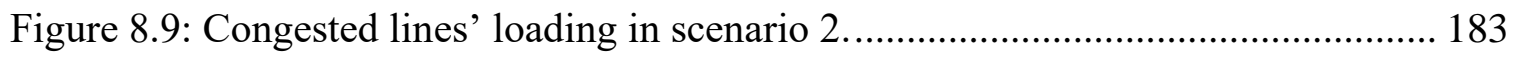

Figure 9.1: Proposed Multicast Routable GOOSE Messages Framework. ................... 187

Figure 9.2: The mechanism of the GOOSE repetition......................................... 188 
Figure 9.3: The test framework configuration.

Figure 9.4: Experimental setup at FIU smart grid testbed.

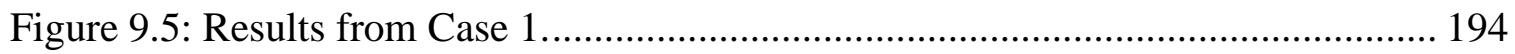

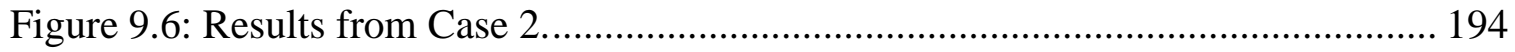

Figure 10.1: Critical communications in power systems and possible threats .............. 198

Figure 10.2: Secure DDS-Based MRGM framework............................................. 198

Figure 10.3: The test framework configuration and network connections. .................. 201

Figure 10.4: Experimental setup at FIU testbed. ................................................ 201

Figure 10.5: Latency measurement for the MRGM framework with Secure-DDS....... 203

Figure 10.6: Bad data injection attack on the MRGM framework .............................. 204

Figure 10.7: SSHA for R-GOOSE message communication .................................... 206

Figure 10.8: DDS-Based Communication Framework for SSHA.............................. 208

Figure 10.9: DDS-based MRGM with SSHA security algorithm. ............................. 209

Figure 11.1: Maximum fault current and its maximum duration time ........................ 213

Figure 11.2: Telecommunication in the power system.......................................... 217

Figure 11.3: Protection System architecture based on IEC 61850 ............................ 219

Figure 11.4: The architecture of the HCPA based on IEC 61850.............................. 220

Figure 11.5: Flowchart of the developed HCPS and the Algorithm of HCPS activation.

Figure 11.6: GOOSE message traffic created by HCPA at Bus 1 ................................ 223

Figure 11.7: GOOSE mapping between HCPA at Bus 1and IEDs............................. 223

Figure 11.8: $400 \mathrm{kV}$ system under study ........................................................ 226

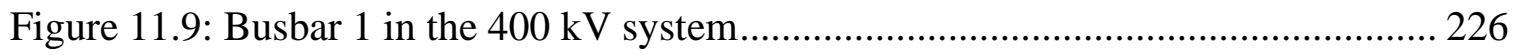




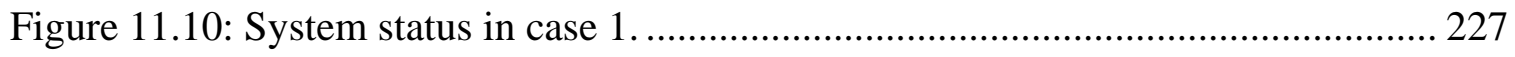

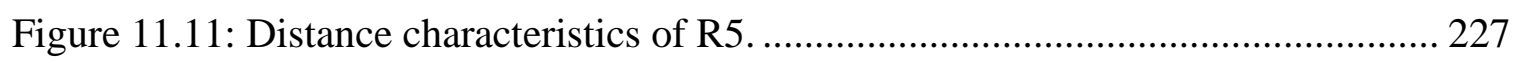

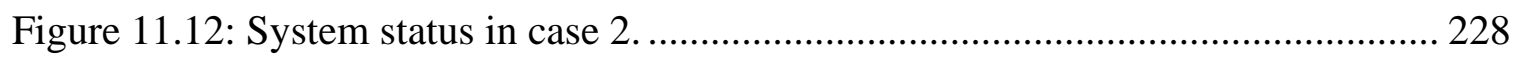

Figure 11.13: Quasi-dynamic simulation Results for Case 2. ................................. 228

Figure 11.14: Quasi-dynamic simulation for Case 3............................................ 230

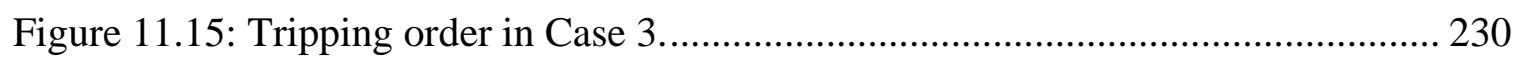

Figure 11.16: Rotor angle of four generators in the system for C2 and C3 $3 \ldots \ldots \ldots \ldots \ldots . . . . . . . .234$

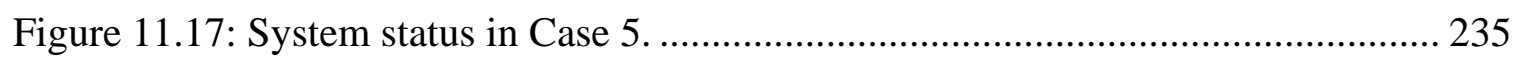

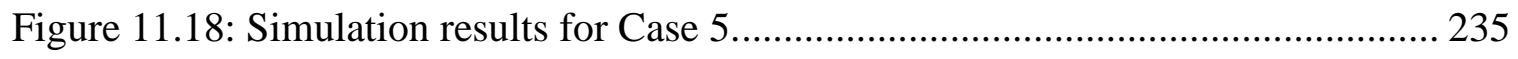




\section{LIST OF ACRONYMS}

ACRONYMS

AANN

AC

ANN

API

APLI

ARP

BC

BC

BMG

CA

CB

$\mathrm{CBF}$

CS

CVT

DA

DAM

DAQ

DC

DCB

DCPS
DETAILS

Adaptive Artificial Neural Network

Alternative Current

Artificial Neural Network

Application Programming Interface

Active Power Losses Index

Address Resolution Protocol

Blockchain

Breaking Capacity

Brooklyn Microgrid

Certificate Authority

Circuit Breaker

Circuit Breaker Failure

Charging Station

Current and Voltage Transformer

DESS Agent

Day-Ahead Market

Data Acquisition System

Direct Current

Disconnecting Circuit Breaker

Data-Centric Publish-Subscribe 


\begin{tabular}{|c|c|}
\hline DDE & Dynamic Data Exchange \\
\hline DDS & Data Distribution Service \\
\hline DER & Distributed Energy Resource \\
\hline DESS & Distributed Energy Storage System \\
\hline DG & Distributed Generation \\
\hline DNS & Domain Name System \\
\hline DP & Domain Participant \\
\hline DPL & DigSilent Programming Language \\
\hline DR & Demand Response \\
\hline EMS & Energy Management System \\
\hline EsCos & Energy Service Companies \\
\hline ET & Emergency Time \\
\hline ETR & Emergency-Term Rating \\
\hline EV & Electric Vehicles \\
\hline FACTS & Flexible AC Transmission System \\
\hline FCL & Fault Current Limiter \\
\hline FCT & Fault Clearing Time \\
\hline FERC & Federal Energy Regulatory Commission \\
\hline FIU & Florida International University \\
\hline FTR & Financial Transmission Right \\
\hline GA & Generator Agent \\
\hline GENCOs & Generation Companies \\
\hline
\end{tabular}




$\begin{array}{ll}\text { GMA } & \text { General Market Agent } \\ \text { GOOSE } & \text { Generic Object Oriented Event } \\ \text { GTDAM } & \text { Game-Theoretic Double Auction Mechanism } \\ \text { HAM } & \text { Hour-Ahead Market } \\ \text { HAM } & \text { Hour-Ahead Market } \\ \text { HBC } & \text { Hierarchical Blockchain } \\ \text { HCPS } & \text { High Current Protection Scheme } \\ \text { HMAC } & \text { Hashed Message Authentication Code } \\ \text { HV } & \text { High Voltage } \\ \text { IC } & \text { Interlinking Converter } \\ \text { IDL } & \text { Interface Definition Language } \\ \text { IED } & \text { Intelligent Electronic Device } \\ \text { IoT } & \text { Internet of Thing } \\ \text { IP } & \text { Internet Protocol } \\ \text { LA } & \text { Load Agent } \\ \text { LAN } & \text { Local Area Networks } \\ \text { LMA } & \text { Local Market Agent } \\ \text { LTR } & \text { Mong-Term Rating } \\ \text { MAS } & \text { Market Clearing Price } \\ \text { MCP } & \text { Microgrid } \\ \text { MG } & \text { Manufacturing Message Specification } \\ \text { HASt System }\end{array}$




\begin{tabular}{|c|c|}
\hline MOI & Microgrid Operating Index \\
\hline MRGM & Multicast Routable GOOSE \\
\hline MU & Measurement Unit \\
\hline OASIS & Open Access Same-Time Information System \\
\hline $\mathrm{OF}$ & Objective Function \\
\hline OMG & Object Management Group \\
\hline OPF & Optimal Power Flow \\
\hline OPR & Optimal Power Routing \\
\hline OSI & Open Systems Interconnection \\
\hline OTC & Over-The-Counter \\
\hline PBFT & Practical Byzantine Fault Tolerance \\
\hline PC & Personal Computer \\
\hline PCC & Point of Common Coupling \\
\hline $\mathrm{P} 2 \mathrm{P}$ & Peer-to-Peer \\
\hline PIF & Power Imbalance Factor \\
\hline PLC & Power Line Carrier \\
\hline PMF & Probability of the Market Failure \\
\hline PMU & Phasor Measurement Unit \\
\hline PoS & Proof of Stake \\
\hline PoW & Proof of Work \\
\hline PRNG & Pseudo-Random Number Generator \\
\hline PSA & Probability of Successful Attack \\
\hline
\end{tabular}




\begin{tabular}{|c|c|}
\hline PSO & Particle Swarm Optimization \\
\hline PST & Phase Shifter Transformers \\
\hline PX & Power Exchange \\
\hline QoS & Quality of Service \\
\hline RES & Renewable Energy Resource \\
\hline R-GOOSE & Routable GOOSE \\
\hline RMSE & Root Mean Square Error \\
\hline RTCM & Real-Time Congestion Management \\
\hline RTM & Real-Time Market \\
\hline RTPS & Real-Time Publish-Subscribe \\
\hline SA & Substation Automation \\
\hline SAS & Substation Automation System \\
\hline $\mathrm{SC}$ & Smart Contracts \\
\hline SCADA & Supervisory Control and Data Acquisition \\
\hline SGS & Seed Generator Server \\
\hline SHC & Short Circuit Capacity \\
\hline SMV & Sampled Measured Value \\
\hline SO & System Operator \\
\hline SOC & State of the Charge \\
\hline SqNum & Sequence Number \\
\hline SRT & Short-Term Rating \\
\hline $\mathrm{SS} 2 \mathrm{CC}$ & Substation to Control Center \\
\hline
\end{tabular}


SS2SS

SSHA

SSL

ST

StNUm

ToU

VD

VDI

VIF

VPN

WAMPAC

WAN
Substation-to-Substation

Secure Sequence Hopping Algorithm

Secure Socket Layer

Short Time

Status Number

Time-Of-Use

Voltage Deviation

Voltage Deviation Index

Voltage Imbalance Factor

Virtual Private Network

Wide-Area Monitoring, Protection and Control

Wide Area Network 


\section{Chapter 1 Introduction and Research Background}

The concept of the electricity market is not new. It was created once Thomas Edison built the first power plant in 1882, and the electric energy was sold to consumers at the market price [1]. Afterward, electrical energy became an original product, and the power industry developed rapidly. By increasing in the number of generation units due to high demand requests, interconnected transmission power networks were created to supply electrical energy with more reliability. For many years, power systems had monopoly structures with high regulations.

The word monopoly means one service provider is in the system. However, from the power market point of view, monopoly happens when one service provider could control at least $25 \%$ of the market [2]. In the monopoly structure, owners could guarantee and maximize their long-term profits. Although the monopoly structure has some advantages, such as avoiding some infrastructure duplication, it has several significant disadvantages, which make it inappropriate for the electricity industry. The most crucial drawback of the monopoly structure is the higher energy price comparing with competitive markets. It is because of this fact that consumers must buy the service from just one provider and do not have any other choices. Besides, quality of service, reliability, and maintenance issues are affected by the monopoly infrastructure, which will lead to low efficient electricity industry and social welfare.

To address these drawbacks, the deregulation in power markets initiated in the early 1980s and deployed by most developed countries [1]. Electricity market deregulation means changing the monopolistic structure into a competitive one, including energy sellers, 
buyers, and service providers, like power transfer over transmission lines. Up to now, many efforts have been made to deploy available analysis methods and rules to power markets [3]; however, it has always been a challenging process to transfer from the traditionally regulated energy market to a deregulated one with new rules and actions. Especially, to evaluate the performance of the competitive power market in terms of energy price, global social welfare, and quality of service, a long-term evaluation, and market rules modification are needed [4]. It is because of this fact that usually in the short-term perspective, deregulated energy markets meet the expected benefits while it should satisfy the long-term expectation. In other words, the main aim of market reform is to decrease the end-user energy cost, but it did not happen in some countries after deregulation. Several reasons have been reported for this issue, such as week competition between market players due to the market structure and the number of players, or not distributing the benefits of the market reform to the related entities such as investors, market players and utility companies. All these issues raised concerns about the effectiveness of the power markets and its expansions to the retail competitions [4]. Furthermore, by penetration of RES, energy storage systems, EVs, and autonomous microgrids, energy markets are facing new challenges in terms of market design and efficiency. Figure 1.1 shows a general view of energy markets and differences between the monopoly and competitive retail electricity markets [4]. 


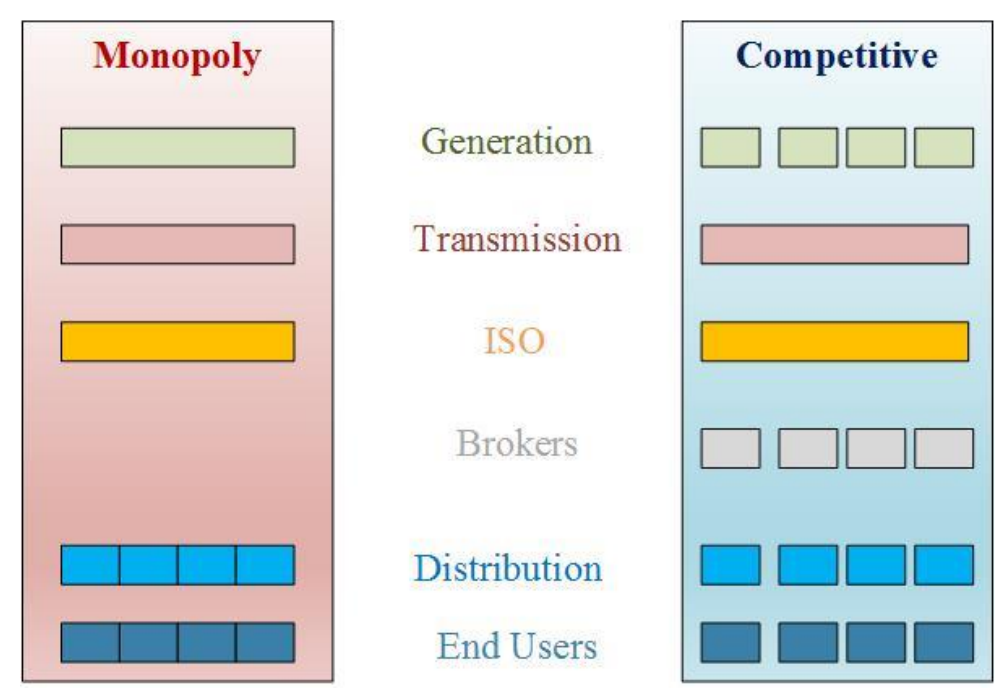

Figure 1.1: Monopoly Vs. Competitive Power Market

\subsection{An introduction to electricity markets}

Electricity markets are different from other products, services, or financial markets due to their physical features, which could not be stored and released to the market immediately. Storing the electric energy is impossible in its original format; we can transfer it to another type of energy to store a limited amount of it and regenerate electric energy once we want to use it. Thus, energy transactions between suppliers and consumers must be scheduled in advance to minimize the real-time energy mismatches. Each energy transaction in the power market contains a seller, a buyer, the amount of energy, the time period and point of energy delivery, and energy consumption in the network [4].

\subsubsection{Electricity market types}

Energy transactions could happen through different types of electricity markets, considering different periods and services. Generally, a liberalized electricity market contains spot and future markets as well as ancillary services and balancing markets. Spot markets include short-term energy transactions with settlement no longer than two days [4]. These markets are designed to fulfill immediate needs. It includes one day, one hour 
or five minutes energy transactions in advance to the real-time operation. For longer energy transactions (e.g., from a week to a year), deals and energy contracts are confirmed through future markets. This type of contract is usually to trade a significant amount of energy between market players over a pre-defined time in the wholesale market.

Besides spot and future markets, there are other types of markets called ancillary service markets. These markets cover a wide range of services in the power market to enhance the reliability and quality of the services. For example, one of the essential ancillary services in the power market is the balancing market. It is because of the risk of energy mismatches in the real-time operation of the power system between actual supply and demand due to inherent load changing and unexpected contingencies in the power system. Imbalances are compensated by spinning reserves in the power system through an ancillary service market. There are many other types of ancillary services in power markets such as reactive power support, frequency control, reserve capacity market, transmission congestion management, etc. Each of these ancillary services has its own rules defined by the regulators and market operators.

\subsubsection{Trading methods in electricity markets}

All transactions in both spot and future power markets can be done using centralized or decentralized market structures. The centralized market is usually known as the pool market, which is run by a trusted third party to clear the market and reports the MCP as well as winners and losers. The pool market is mostly used for short term contracts such as day-ahead, hour-ahead markets, and real-time markets. The decentralized market contains bilateral OTC contracts directly between two parties for long-term (e.g., several weeks, months or years) contracts and usually with the help of a broker. 


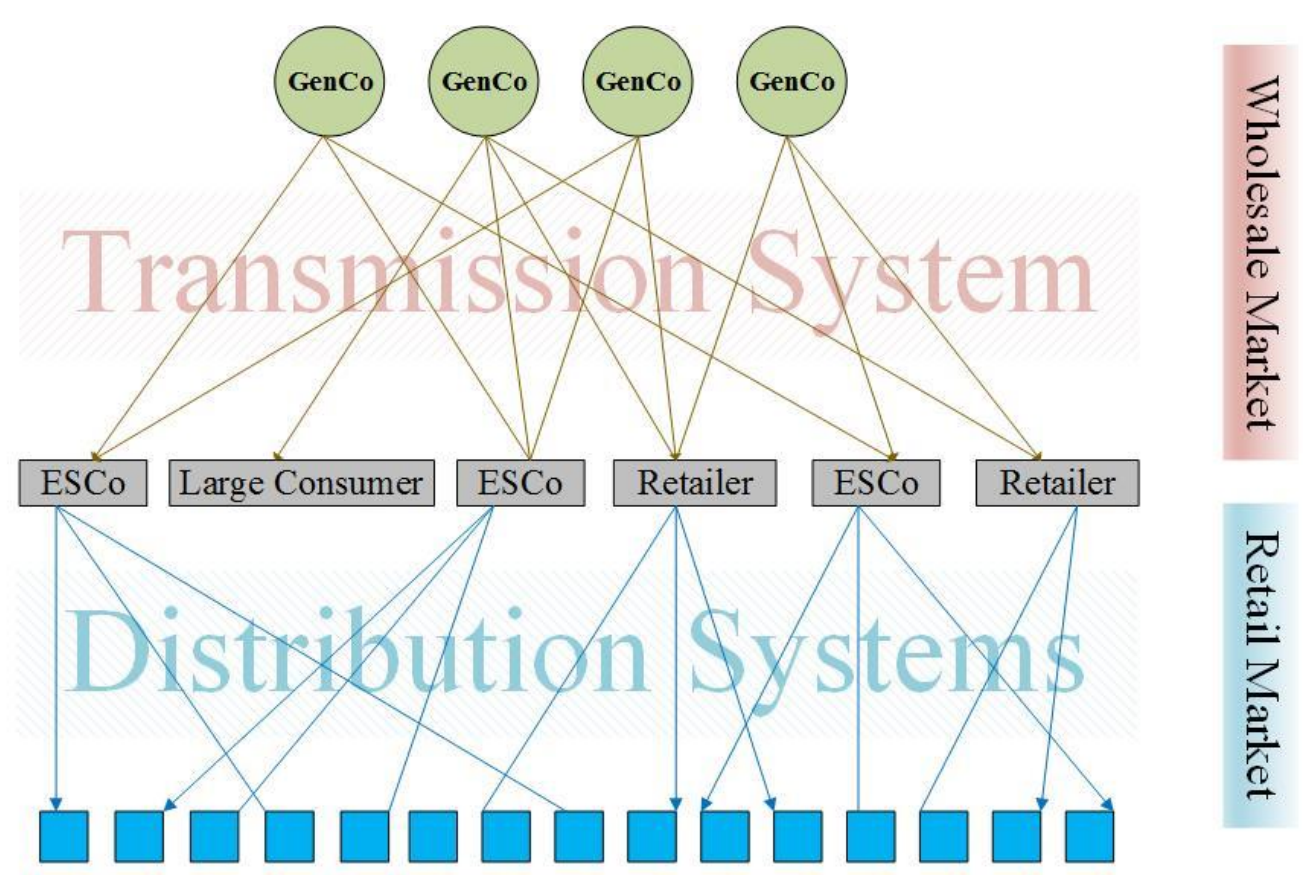

\section{Customers}

Figure 1.2: Wholesale and retail electricity markets

\subsubsection{Wholesale and retail electricity markets}

The wholesale electricity market addresses the bulk energy transactions in power systems between GENCOs and retailers and large end-users through centralized or decentralized markets [5]-[6]. The competition between GENCOs for selling energy to maximize their benefits and retailers for buying electrical energy and minimizing the energy cost might cause lower energy price comparing with a non-competitive energy market. However, retailers also participate in the retail markets to sell electricity to endusers, which could also be a highly competitive market regarding the energy tariff, policy, and services which they recommend to the end-users. Usually, EsCos do not charge the customer with a real-time energy price. They have different tariffs and pricing methods such as ToU, Fix tariff, or participate in demand response programs [7]. Figure 1.2 shows an overview of these electricity markets [5]. 


\subsubsection{Benefits from power market deregulations}

There are several expectations from a well-developed competitive electricity market, including [4]:

- Lower energy price due to competition and optimum allocation of resources: An actual competition between market players inherently decreases the energy price. Furthermore, reducing the energy transfer cost by optimal allocation of energy resources could significantly reduce the energy price.

- Minimizing the investment costs: When private companies and investors want to participate in power markets, they always try to minimize their investment cost by conducting comprehensive researches.

- Improving the system efficiency: In an open access and competitive electricity market, reducing the service cost for the service provider means more capabilities and consequently, more benefits from the market. The energy consumption in more efficient systems is lower for the same amount of output. Therefore, by the same selling price, a more efficient system gets more benefits.

- Decreasing the pollutions due to regulation: Market rules and regulations could profoundly affect the pollutions due to generating the electric energy.

- Developing and deploying new energy resources: New energy resources like solar and wind energies are free for consumers while they can sell electrical energy in the market. During recent years, market regulators have had very intensive programs to motivate investors to participate in this field, which also led to decreasing the investment cost for renewable energy resources and made an advancement in these technologies. 
Therefore, competitive and deregulated electricity markets could have significant advantages for all market participants. However, there are always many challenges that need to be addressed accordingly.

\subsection{Challenges in electricity market deregulations}

Moving from the traditional to modern electric energy regulatory causes significant challenges for both energy policymakers and system operators. It is because of the uncertainties in investments, privacies, and deregulation impacts on customers during the transition process as well as new structures of modern power systems. In this section, we describe these challenges, which can be divided into two categories; regulatory problems and modern power system challenges.

\subsubsection{Regulatory challenges}

Regulatory challenges and uncertainties for investments and active participation in the new electricity market structure with new rules could cause significant reliability problems for service provides and consequently for end-users. These challenges should be addressed correctly to minimize regulatory reform risks. One of the critical issues is called unbundling. It means that despite the monopoly operation of the power market, in deregulated power markets, competitions for energy trades must be separated from energy transfer service by transmission networks, which will cause competition to get access to transmission capacity. Many regulatory challenges are created, such as proper rules to eliminate the transmission system access discrimination and provide an open-access environment. This is a complicated challenge because not only the benefits of market players should be considered, the transmission system owners also must be satisfied with new rules. There are many methods and solution in this regard which used by different 
energy markets such as FTR, nodal pricing, etc. [8]-[9]. Furthermore, market regulators should also consider the regulatory issue due to empowering the end-users, supply security, social goals as well as environmental protection. In other words, the potential of end-users to choose different suppliers and data security and nondiscriminatory market structure are other regulatory challenges for deregulated electricity markets.

\subsubsection{Modern power system challenges}

Not only the wholesale energy market should be reformed correctly, but also the retail markets must be effectively changed to address the needs of modern power systems. These needs are due to the new structure of power systems, mainly due to the creation of active distribution networks [10]. In advanced power systems, RESs, DGs, mobile loads (EVs) and autonomous microgrids have been widely integrated into the power systems, and the trends show that they will be used more in the near future. Besides all technical issues in

power system operation and dispatching the energy resources such as uncertainty of renewable energy resources, short service period, etc. the market challenges also raised due to the intention of these resources to participate in electricity markets. Therefore, a necessity raised to effectively expand electricity markets to end-users' level to address the open-access market, market liberalization and required ancillary services.

\subsubsection{Communication challenges}

Modern power systems extensively depend on communication systems. It can be seen in all aspects of power system control, protection as well as electricity markets. The communication for each purpose has its own requirements, limitations, and tools. In a power market, the structure of the market and trading mechanism defines the communication framework. For example, in a centralized power market with a trusted 
third-party, all agents in the market need to communicate with the leading agent. This communication platform is simple and mostly used for wholesale energy markets. However, by increasing in the number of market players and the necessity of open access markets, local markets were created to trade energy within different areas without communicating with the higher-level market agents. As a result, the communication system should be changed to provide fast, reliable, and secure data exchange capabilities for all agents within an area. Regarding different communication protocols, standards and unique features of each one, in-depth research is required to choose an appropriate communication framework for modern power systems.

\subsection{Microgrids in energy markets}

In this section, we describe the MGs and their participation in deregulated electricity markets. For this aim, at first, we present the concept of MGs and the configuration of active distribution networks. Afterward, the interconnection of MGs is described following by their technical and economic advantages. Finally, a comprehensive literature review of existing electricity markets for microgrids is presented.

\subsubsection{Active distribution network}

Traditional distribution networks mostly have a unidirectional form, which is called passive networks. It means that power flow in this network is always from utility suppliers, usually located in power transmission systems. The electric energy is being released to the high voltage transmission network, passes through medium voltage sub-transmission systems, and finally goes to low voltage distribution networks to feed end-users. Nowadays, with the creation, installation, and implementation of DGs and clean energies with renewable DERs in low voltage systems, it is possible to inject electric energy from 
the low voltage system to the utility grid. A distribution network with the bidirectional power flow capability is called an active distribution network. Although it has many advantages like local energy generation and control, reduce energy losses through transmission systems, etc., there are many technical challenges and concerns with active networks. The active distribution networks need a flexible, intelligent, and highly coordinated protection and control schemes [11]. The interaction of an active distribution network with the utility grid is one of the most challenging technical and regulatory issues. In these networks, advanced measurements and sensors are needed along with a welldeveloped network management system, adaptive protection schemes, and intelligent control methods [12].

\subsubsection{Microgrids}

A microgrid is defined as a small-scale low voltage electricity network with a local group of electricity resources and loads connected in a geographical area. Microgrids usually integrate various DGs, especially RESs and converted based DERs [13]-[14]. There are two operation modes for Microgrids [15]; Islanding mode, where Microgrid is operating autonomously and is not connected to the external grid.

In this situation, the Microgrid needs to provide all required services by itself and will not be supported by the utility grid. On the other side, there is a grid-connected mode for MG's operation. In this mode, the MG is synchronized and connected to the external grid and can get essential services like frequency and voltage support from the main grid and exchange the energy with the utility grid. Therefore, MGs are in the category of active distribution networks. The islanding mode of MG is suitable for supplying areas that do not have access to the main power grid. The grid-connected mode also has some advantages 


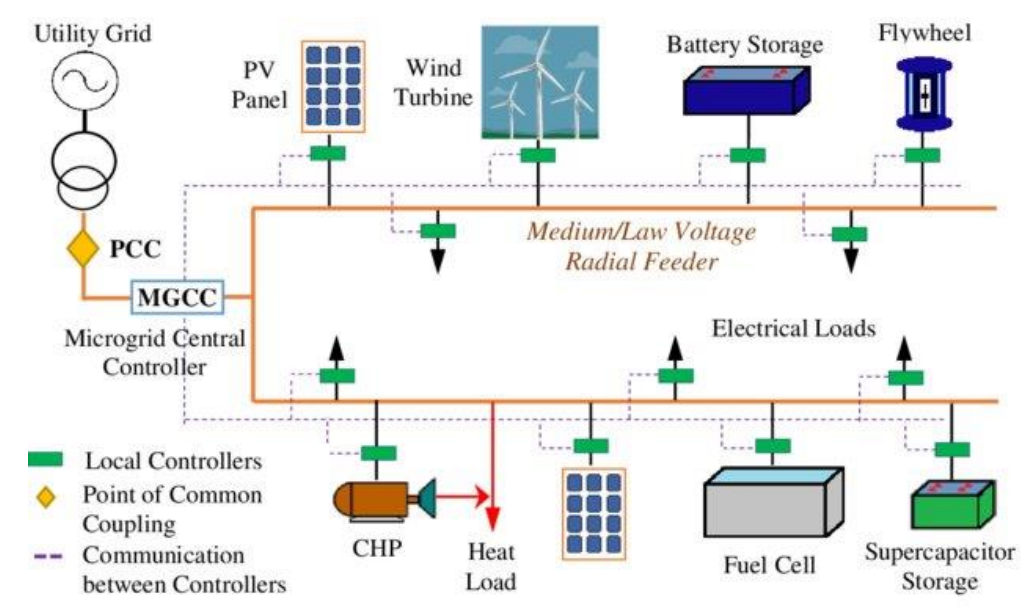

Figure 1.3: Configuration of a typical MG [16]

for the power grid. In the grid-connected mode, the MG can be considered as a controllable entity of the system; for example, a controllable load/generator in the system. This feature can improve the demand response programs and ultimately reduce power transmission losses and provide local reactive power support. From a customer point of view, it can provide an independent, uninterruptable, and locally reliable and efficient power supply for end-users. The social and environmental benefits are less pollution and global warming due to using clean energy resources.

Figure 1.3 shows a typical microgrid containing micro resources and loads in an LV distribution network. A standard MG is connected to the external grid through its PCC by a CB. The most challenging issue in MG operation is switching from one operating mode to another one. For example, if MG is in grid-connected mode and due to any disturbance in the external grid wants to switch to islanding mode, first of all, the islanding detection algorithm must be activated to detect the disturbance and the necessity of switching through and effective algorithm [17]. Afterward, the major challenge is autonomous control of the MG, because by islanding detection and switching, the MG is responsible for the stability, power balance, quality of service, and uninterruptible supply for its priority loads. All these 
issues must be addressed correctly during this transition. The control algorithm needs to switch off unnecessary loads and deploy proper control algorithm to keep the MG stable and balance the supply and demands.

Another critical challenge is the protection of MG. As mentioned before, the MG is an active distribution network with bidirectional power flow feature. It makes difficulties for the protection of this system [11]. Islanding detection and switching to islanding mode is also another protection challenging issue [17]. It is because of this fact that by losing the main power grid, fault detection in MG is very difficult due to its low SHC. The SHC detection, feeding, and protection needs an adaptive protection strategy which highly depends on the communication system [12].

\subsubsection{Interconnection of microgrids}

MGs are naturally designed to use micro-energy resources and clean energies to feed local loads. Therefore, their maximum capacity is approximately around 10 MVA [12]. However, the interconnection of MGs in an area and creating a power pool will allow us to feed larger loads by several MGs through the distribution network. For this aim, all MGs are connected to the network, and load is split among them using a control and energy management system considering the capacity and capabilities of all MGs. However, the energy transaction among MGs need a competitive and well-organized power market.

\subsubsection{Microgrid power markets}

The first electricity markets had a centralized framework with large power plants located in the transmission system and energy consumers in different locations of the system. Nowadays, due to the high penetration of DGs and RESs along with DESSs in distribution networks and creation of microgrids in power systems, decentralized energy 
systems are used to run the energy markets locally [18]. Therefore, a need arises for the development of an optimal open-access electricity energy market in the distribution network. For a market framework to be practical, it should consider the autonomous operation of individual microgrids and the inter-microgrid market, not only to minimize the dependency on the network in the grid-connected mode but also to avoid load shedding in the islanding operation mode. In both grid-connected and islanding operation modes, the interaction among microgrids can increase the system's reliability, reduce the energy cost and energy losses [19]. Running the local markets helps the local communities to be independent and use their local generation with lower energy prices [20]. It makes it possible for microgrid's costumers to get access to their neighbors' electric energy in a local market using P2P energy transactions [21].

Additionally, social factors are essential. In [22], Bertsch et al. have discussed the public acceptance of using local RESs. In [23], developing a shared vision with affected communities has been studied to show the increasing public acceptance of local microgrid energy markets. Although the optimal operation of microgrids and feasible connections of multiple microgrids have been studied extensively, there are still potential to research in the fields of microgrid energy markets. In [24], Lamparter et al. proposed a flexible market structure to coordinate prosumers, consumers, and suppliers. The detailed mechanism of the local energy markets is described by Blouin and Serrano [25], where they introduced a P2P decentralized energy market with randomized buyers and sellers. In [26], a double auction mechanism was investigated for energy pricing and local energy product allocation. The concept of the continuous double auction which takes the transmission congestion constraints in pricing algorithm was introduced in [27]. 
Market agents and their interactions must be defined before establishing an actual microgrid energy market. For this aim, the concepts of multiagent-based microgrid market structure and inter-microgrid transactions have been studied in several references. For example, in [28] and [29], applications of MASs in electricity markets were reported for the optimization of the retailer portfolio and the energy management in microgrids, respectively. In [30], a MAS which provides the DR service from residential end-users was developed. An intelligent load management solution was presented in [31] to mitigate the energy imbalances in microgrids using a virtual market that allows energy transactions between microgrids. In [32], the authors tried to decrease the energy exchange between microgrids on one side and the utility on the other. The purpose of minimizing loss and cost in distribution systems comprising several microgrids was presented in [33]. A gametheoretic algorithm to manage the energy flow and a decentralized energy exchange approach among microgrids was presented in [34] and [35], respectively. In [36], a multilayered multiagent based EMS was introduced to manage the energy inside and among microgrids using distributed generators and storage systems.

The implementation of MAS in microgrid market operation along with the concept of microgrid market design have been widely discussed in the literature. For example, in [37], a pool market for energy management of a microgrid has been introduced, in which the microgrid actively responds to change in electricity price by scheduling its controllable resources. In [38], a noncooperative and competitive game theory approach were proposed for energy-trading between microgrids. In [39], an aperiodic reward-based energy market model has been presented, in which consumers ask for energy and respond to the posted rewards regarding their requirements. Providers also respond to the posted awards utilizing 
changing their energy bids. In [40], a multiagent approach for the energy auction market design has been proposed which includes the effect of storage systems in an interconnected microgrid area and their impacts on grid losses.

In [41], a MAS based energy framework for smart microgrids was presented, where the authors proposed to divide the system into several control areas and utilize a MAS to control and monitor the system. Afterward, an overall optimization algorithm is run in an upper-level agent, where all demand and generation flexibilities, limits and constraints, and the benefits/costs of all components are considered. A similar approach using a hierarchical energy management strategy was presented in [42]. Although the developed optimization in the upper-level agent may lead to finding an optimum solution, the optimization solution time is high because a central solver requires solving this optimization problem, including all system and components' constraints, demand flexibilities, and generation availabilities. Hence, the authors of [41] did not consider the participation of DESSs and DGs in the intermicrogrid market to remove the non-linear equations from the optimization problem and, therefore, reduce the optimization solution time. However, in a real open-access environment, all components should be able to participate in the market to increase the system's flexibility and reliability and reduce the operation cost. In [43], a reverse auction model was deployed for energy providers in one MG to serve a lumped load in each market interval of the hour-ahead market. However, in this reference, the authors did not consider mismatches caused by load variations, nor did they consider DR in their auction model.

Moreover, in [41] and [43], the HAM was presented as a real-time market. This means that any forecasted mismatch after running this market will not be optimally mitigated, the thing which may cause unforeseen technical problems, load shedding, and high energy 
cost. In addition, the controlled area will be more dependent on the utility for energy mismatches. Regarding the literature, a very well-organized competitive energy market structure that optimally mitigates any power mismatch through a fast optimization method is needed. To the best of our knowledge, the literature lacks a framework that is fast enough for real-time market operation of a multi-microgrid system.

\subsection{Modern electricity markets}

Although the pool markets are well developed and used in competitive electricity markets, bilateral energy transaction frameworks are under consideration of market players in modern electricity markets. It is because of the creation of new trading technologies for financial trading and their unique features that motivate other industries to deploy them. For example, by developing and implementing the $\mathrm{BC}$ technology for secure $\mathrm{P} 2 \mathrm{P}$ financial transactions in 2008 [44], this technology has been used in many other applications afterward. Although every new technology has some highlighted advantages, they can contain several disadvantages as well. Choosing and implementing new technology in a new form need a comprehensive evaluation of all features and challenges. Furthermore, to adopt new technology for a new application, it is necessary to modify it based on the application requirements.

\subsubsection{Blockchain technology}

The BC, also called the distributed ledger, is a technology that was first developed in 2008 by Satoshi Nakamoto as a new technology for distributed peer-to-peer transactions aiming at maintaining the transactions' order and avoiding the double-spending problem [44]. In the BC technology, network nodes, which are called miners, are responsible for creating and linking a robust and auditable chain of transaction data blocks in chronological 


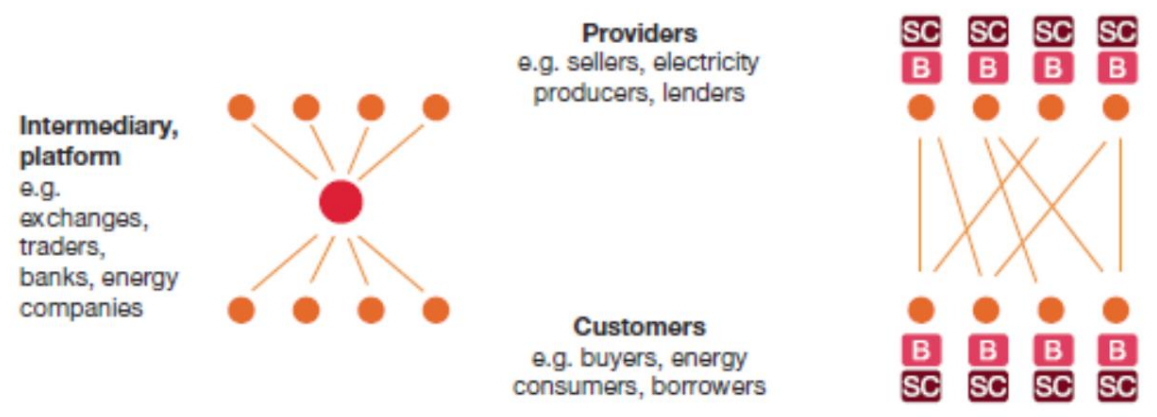

Figure 1.4: Centralized and distributed transaction models [45]

order using the hash function [46]. The BC technology introduced a new decentralized and fully distributed transaction platform without needing a trusted third, which is used for traditional centralized structures. This technology provides a high level of security, transparency, and robustness [47]. Because of all these features, more than 1900 BC-based cryptocurrencies have been developed and deployed. The increase in the number of digital currencies may cause interoperability problems in the near future [48]. In addition to implementing the $\mathrm{BC}$ for financial transactions, the $\mathrm{BC}$ technology is highly being used in other industries and fields [49].

\subsubsection{The concept of the Blockchain}

The $\mathrm{BC}$ technology is based on the distribution of databases among all participants within the $\mathrm{BC}$ network [50]. This database or ledger contains all pre-approved transactions/data in the system approved through a consensus algorithm. It is worth mentioning that once a block is built and verified, it is impossible to erase it afterward. In other words, each participant in the BC network can directly trade with other network members without the participation of trusted third-party [45]. The innovation, main feature, and advantage of the $\mathrm{BC}$ is its distributed database, which removes the need for a central 
database by sending a synchronized copy of the ledger to all network participants [51]. A blockchain benefits from a chain of unchangeable transaction data blocks within a distributed structure with highly mitigated risks of double spending in the market [52]. Each participant in the $\mathrm{BC}$ runs a consensus protocol to validate transactions and make a block of data. The new block is chained to previous blocks using a powerful cryptographic mechanism, which is called Hash function [53]. Figure 1.4 shows a traditional centralized transaction model as well as the $\mathrm{BC}$ transaction model.

Despite the traditional model with a centralized database and third-party agent, in the $\mathrm{BC}$ technology, peer-to-peer transactions are possible between participants, and each one has a copy of the blocks. Furthermore, in the BC model, all transactions are made based on SC and there is no need for a trusted third party [54]. Based on the definition in [55], the $\mathrm{SC}$ is " a computerized transaction protocol that executes the terms of a contract." An SC is an agreement between all participants within the BC network. It is an executable script stored in the $\mathrm{BC}$ for safe and correct transactions using computational protocols and without needing a trusted third party. This code is then activated and implements different possible actions once a transaction is initiated to its address.

\subsubsection{Three generations of the $\mathrm{BC}$ technology}

Generally, the BC technology could be categorized into three categories regarding its capabilities [49] and [56]:

1. Blockchain 1.0: It is the first generation of blockchain, which is used for digital cryptocurrency transactions like Bitcoin, token forms, and secure private transactions.

2. Blockchain 2.0: The second generation of the BC was developed based on SCs for 
applications beyond the cryptocurrency transactions. The Ethereum transaction platform is an example of this generation which supports SCs using automated codes in the chain.

3. Blockchain 3.0: The new generation of $\mathrm{BC}$ technology is used in areas beyond the previous two generations. It is deployed for some applications in government, health, science, energy, and IoT. This generation uses SCs concept for decentralized organizations with a high degree of autonomy and special rules.

The BC technology and its role in some applications have been reviewed in the literature. For example, The BC for data-intensive applications, IoT and big data management were presented in [57] and [58], respectively. In [59], the security of the BC was discussed while its capability for decentralization and P2P framework was presented in [60]. Many technical aspects of the BC technology such as consensus protocol, SCs' vulnerabilities, the size and usability of the $\mathrm{BC}$ along with data integrity, scalability, security, and privacy have been studied in [61].

\subsubsection{Blockchain in different applications}

The applications of the $\mathrm{BC}$ were usually classified into financial and non-financial groups [50]. It is because of this fact that the $\mathrm{BC}$ was first developed and deployed for financial transactions in the Bitcoin system and then, many other cryptocurrencies were developed. Although the applications of the $\mathrm{BC}$ in non-financial industries are still in early development stages, due to its vast areas of implementations, it can be classified into the following categories, as shown in Figure 1.5. 


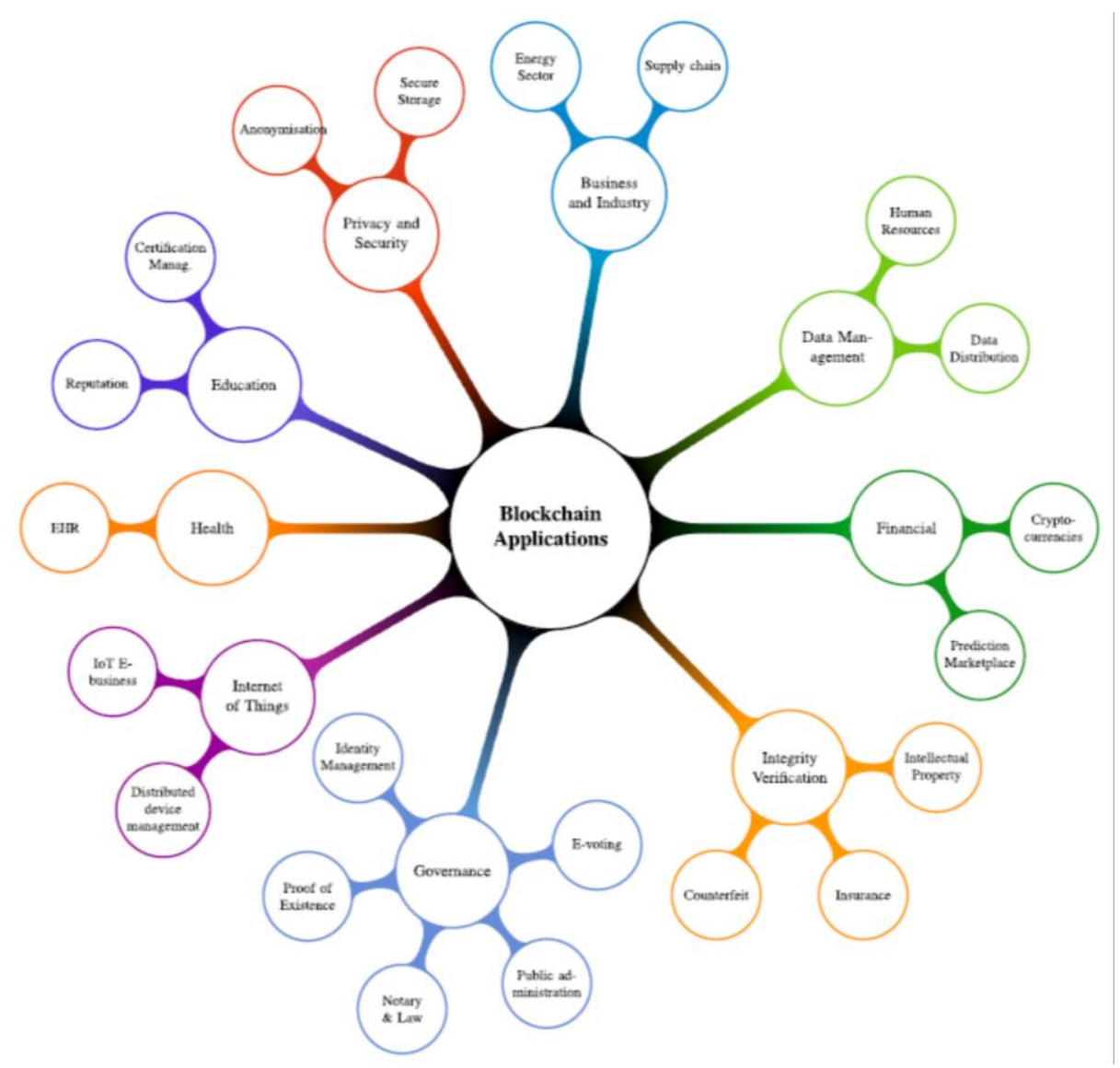

Figure 1.5: Applications of the BC technology in different fields [62]

\subsubsection{Financial applications}

As mentioned before, the $\mathrm{BC}$ technology has been widely used for financial applications such as cryptocurrencies, financial transactions, and business services, fiat money, derivative contracts and trade, digital payments, loan management, etc. [63].

\subsubsection{Governance}

Keeping official public records (e.g., identification \& registration, attestation, marriage certificates, contract, taxes, votes, and any other legal documents) in different formats form people and organizations for a long time is a challenging issue for governments. The BC technology could help to address this challenge [64]. 
The $\mathrm{BC}$ can provide a secure integrity platform for social infrastructures and devolve a distributed and efficient database. In [65], the world criticizes the project has been presented as an example for BC-based passport service, which can identify citizens all over the world. It can address the lack of identity documents, especially for refugees and immigrants, those who their governments usually refuse to issue their certificates. In these situations, the $\mathrm{BC}$ technology is a powerful technology to provide reliable identity data to worldwide citizens [66]. Beside all these services, nowadays, governments are interested in using $\mathrm{BC}$ technology in the public sector, specially to authenticate persistent documents [67]. Furthermore, the electronic voting (e-voting) mechanism has been activated during the last years to speed up, simplify, and reduce the cost of the voting process [68]. Since most of the e-voting mechanisms have a centralized structure and trusting on the third party is always a concern for everyone [69], several decentralized voting systems have been proposed to enhance the decision-making process [70]. Since the BC technology can offer an open-source, independent and highly secure network for voters and election organizations, it has been considered for new e-voting systems [71].

\subsubsection{Healthcare management}

The healthcare industry could be profoundly affected by the BC technology in several areas such as management of public healthcare, patient medical and treatment records, and any related data [72].

\subsubsection{Business and IoT}

Recent researches show that a dramatic increase happened in the data recently due to population growth and the advent of IoT [73]. On the other side, nowadays the BC technology is developing fast, and implementing the decentralized approaches for IoT is 
under study. Therefore, the relationship between these two domains is under consideration and attention because the $\mathrm{BC}$ may enhance the performance, security, scalability, interoperability, data exchange, and management of IoT [74]. Furthermore, the BC technology has a significant potential to be implemented for business applications [75]. Nowadays, many business models are emerging with IoT, where the BC is highly deployed. In the near future, it is expected to link IoT devices with cryptocurrency accounts [76]. It will result in recording all microtransactions. It could also happen in the energy sector and especially for smart grid applications and electric energy trades customers [77]. Supply chains, transportation services, inventory management, wireless sensor networks, and many other applications are using the $\mathrm{BC}$ to enhance the security, efficiency, and performances [78].

\subsubsection{Security, data management and education}

The security issue of big data, including personal and sensitive formations as well as scalability and mining processes, could be addressed by relying on the BC technology [79]. There are many examples to show the effect of $\mathrm{BC}$ on the security enhancement of big data applications. For instance, in [80], an open-source BC-based platform was developed for a decentralized DNS with significant security, efficiency, and privacy features. In addition to privacy and security, data management is one of the most critical applications of the $\mathrm{BC}$ [81]. For example, in [82], the $\mathrm{BC}$ has been implemented for cross-organizational workflow management, and results showed the potential of the $\mathrm{BC}$ for this infrastructure. For education purposes, the BC could help us in learning environments by starting educational documents and records as well as students' achievements, certificates and credit management, etc. [83]. 


\subsubsection{Industrial applications}

A significant increase in using the $\mathrm{BC}$ technology in industrial applications such as supply chain management as well as the energy sector is expected. The supply chain could benefit from more flexibility and visibility if the BC technology is applied [84]. The BC can decrease paper works, facilitate origin tracking and transactions, enhance the system security and robustness of contract management mechanisms, food safety, IP protection, advanced data analytics and better customer service across complex supply chains [85].

$\mathrm{BC}$ also has an enormous impact on the energy sector by affecting energy transaction platforms and processing [86]. Using the BC technology, the energy cost may be reduced while new marketplaces and business models are coming on the table with more capabilities to manage complex systems, enhance the security and motivate energy markets to create energy communities especially for electricity markets [87].

\subsubsection{Blockchain in electricity markets}

In addition to all advantages in using the $\mathrm{BC}$ technology in industrial applications, this technology could significantly have an impact on electricity markets through enhancing P2P trades between market players, supporting the smooth operation of power systems, better handling of demand response programs, more efficient billing process and transaction processing schemes [88]. With the development and penetration of green energies and renewable resources in power systems, some identification challenges also created which the BC technology could address them accordingly, such as issuing certificates of origin [89]. Furthermore, it could solve the high penetration of electric vehicles in electricity markets and provide $\mathrm{P} 2 \mathrm{P}$ energy transaction schemes in a decentralized platform [90]. 
Energy transactions within Microgrids are considered as community energy markets, where microgrid entities are market participants. Up to now, researchers have proposed different types of BC-based energy markets for microgrids. For example, implementing the BC technology in energy markets was defined at first by Mihaylov et al. [91], where they introduced a new virtual currency for energy transactions. This currency is the translation of energy generation/consumption in the systems, and its market value is centrally determined by the distribution system operator [92]. A local BC-based market model was developed by Al Kawasmi et al. [93] for carbon emissions trades aimed to facilitate anonymous trading between the market players. This concept was more developed by Aitzhan and Svetinovic in [94], where they introduced a token-based, decentralized, and private energy-trading platform. By running different scenarios of P2P transactions and evaluating data integrity and security, they concluded that the BC technology could be used as a decentralized platform for energy markets with a high degree of security and privacy.

A small-scale BC-based electricity market for machine-to-machine energy trades was developed by Sikorski et al. [54], where they concluded that the BC could be successfully used for power markets. The Brooklyn microgrid (BMG) is an actual case study in New York, where the BC-based energy market developed and implemented across three distribution networks by LO3 and Siemens companies [95]. The distribution network in this area is highly congested and experiences many environmental events such as severe weather events, hurricanes, etc. On the other side, a significant increase observed in renewable generations in this area as well as EVs and energy storage. Therefore, the BMG was created, and a private BC-based local energy market was developed to address these 
challenges. This market was run for a three-month trial, and many regulatory and legal challenges were investigated.

\subsubsection{Challenges of using Blockchain in power markets}

Besides all the advantages and benefits of developing and implementing the BC-based energy markets, Blockchains are still an emerging topic and technology with numerous regulatory and technical challenges as listed below:

- Scalability of the BC energy market and its limited transaction loads [96].

- The complexity of the $\mathrm{BC}$ technology for implementation in the various system as well as the energy industry [97].

- The risks of distributed ledger and public accessibility in energy markets [98].

- The energy consumption when a BC-based market is implemented [99].

- In addition to all the challenges mentioned above, there is another technical challenge in implementing the BC-based electricity market. This challenge is about the feasibility of energy transactions over the transmission, sub-transmission, and distribution networks. It is because of this fact that despite most of the financial/non-financial transactions, the electric energy transactions must be physically possible between buyers and sellers. In other words, energy is passing over the power transmission system to feed the loads.

Therefore, for a transaction to be approved between two parties in different locations

in the network, power system constraints must also be evaluated to check the feasibility of each transaction. In this dissertation, we addressed the transaction feasibility check for inter-microgrid transactions in a multi-microgrid system by developing and verifying a hierarchical BC-based energy transaction framework. 


\subsection{Ancillary services}

In the deregulated electricity market, customers have more power to control their resources. Therefore, the power system operator is losing its power in controlling all resources as required to guarantee the reliable operation of the system, which is the main ability of the utility to respond to dynamic demands. Ancillary services such as real-time transmission congestion management, demand response and reactive power control are some solution for this problem in the deregulated power markets [12].

Furthermore, ancillary services are necessary services in a power system for safe, reliable, and stable operation. There are many ancillary services in power markets such as spinning and operating reserves, frequency control, voltage, and reactive power control, load following, scheduling and dispatch, system protection, congestion management, energy imbalance, etc. It is worth mentioning that in traditional power markets, just the supply authority was responsible for the ancillary services. However, in modern power markets, energy consumers and MGs owners can provide ancillary services. Therefore, the role of the utility companies in energy markets must be defined when MGs could work autonomously. As mentioned in [100], utilities can revise their business model and provide energy balance with ancillary service to support MGs. Among all above mentioned ancillary services, in this dissertation, we are focusing on two ancillary services in smart grids: (1) Real-Time Congestion Management and (2) Energy imbalance service.

\subsubsection{Real-Time Congestion Management (RTCM)}

The RTCM is defined as real-time remedial actions to eliminate congestions from power transmission systems securely [101]. The deregulated structure of power systems also makes it essential to manage real-time congestions through an open market, where all 
market players can participate in ancillary services. Although SOs always try to manage the congestions in day-ahead and hour-ahead energy markets, real-time contingencies are still probable during the operation time. Since power systems are almost highly loaded due to daily demand increments, real-time contingencies could cause insecure situations from the operational point of view. These situations are called real-time congestions, and all remedial actions which are done to prevent the system from collapsing are called RTCM. An effective RTCM method should be able to optimally remove the congestion by using all power system capabilities and guide the system to a new operational point considering all power system constraints [102]. Since the number of independent microgrids with high generation capacity is increasing in the modern power systems, participation of these resources in the RTCM problem thorough a competitive ancillary service market is considered by the system operator.

There are some cost-free and non-cost-free tools in power systems for this purpose. Using the capability of FACTS devices and transformers' tap changers that could be considered as cost-free methods. At the same time, generation rescheduling, and demand response program are called non-cost-free methods for the RTCM problem [103]. Usually, the RTCM problem consists of an optimization problem aimed at minimizing the congestion management cost. For example, in [104], an optimal rescheduling scheme has been proposed based on the PSO algorithm. The proposed method calculates transmission lines' sensitivities for generators' active power variations and uses these values to minimize the RTCM cost regarding the generators' bid prices. 
In [101], a comprehensive RTCM algorithm has been presented. This algorithm uses the quasi-dynamic thermal rating of transmission lines to increase transmission system thermal capacity and adopts a load shedding program in critical congestion situations, where rescheduling is not able to manage the congestion independently. Although realtime constraints (ex. Generators' ramp rates and congestion clearing time) have been modeled in the RTCM problem, the cost-free methods have not been modeled in the proposed RTCM formulation. Furthermore, only classical methods have been used to solve the optimization problem. Therefore, it is highly probable that the solution algorithm finds just a local solution for the challenge. Besides that, the developed algorithm is aimed at removing the congestion from the transmission system during a pre-defined clearing time considering the amount of initial congested line current in the post-contingency condition.

Although the algorithm divides the congestion clearing time into subsequent subintervals to evaluate the power system variations during the rescheduling process, it does not consider the thermal adaption of conductors during the RTCM process. In [102], PST have been modeled in the RTCM problem as a cost-free method. Results showed that the incorporation of PSTs' operation in the RTCM problem not only could reduce the RTCM cost but also in some critical congestion cases, the feasible solution for the problem is not available unless PSTs participate in the RTCM problem. Authors have used the PSO algorithm to solve the RTCM problem in an appropriate solution time, the PSO's parameters have been determined based on the trial and error method, which is not a suitable method for real-time applications. Therefore, a fast and reliable optimization algorithm is needed for the RTCM problem in the smart power system in dealing with the congestion situation. Furthermore, the RTCM model should be well developed to optimally 
use the available thermal capacity of transmission lines for the RTCM problem and minimizes the congestion costs accordingly.

In this dissertation, an adaptive RTCM algorithm is developed considering the thermal adaption of transmission lines to manage the congestion optimally. In this method, the demand DR is modeled in the RTCM algorithm to create an actual RTCM market, where all market players would be able to participate in it. Developing a hybrid real-time search algorithm as a powerful solution tool for the RTCM optimization problem, including an AANN and a modified PSO algorithm, is another issue which is discussed and developed in this dissertation.

\subsubsection{Energy imbalance service}

Due to the high penetration of DESSs, RESs, and different types of DC loads in microgrids, there are AC and DC buses in microgrids that form hybrid AC-DC microgrids [105]. This type of microgrid has been studied in literature from different points of view, such as power flow algorithms, optimal dispatch, and control issues [106]-[107].

In hybrid AC-DC microgrids, the connection of AC and DC systems is possible through Interlinking Converters (ICs). Therefore, several control schemes have been developed for these ICs aiming at optimal DC load sharing between ICs or improving voltage quality, as described in [108] and [109], respectively. However, in most cases, due to unbalanced load conditions in the AC system, the microgrid is operated in unbalanced conditions [110]. Generally, there are two types of hybrid AC-DC microgrids; the first one includes one single DC bus connecting all DC loads and generations where this bus is linked to the AC system by one or several ICs. The second type is the situation where there are several separate DC buses in the system that each one is connected to the AC system by its own 
IC. In both types of hybrid AC-DC microgrids, unbalanced three-phase loading conditions would create significant problems for the optimal operation of the microgrid.

In the literature, several studies have focused on these issues, especially in the islanded operating mode of microgrids. For example, in [110], a dynamic power routing strategy has been proposed for islanded operating mode of a type one unbalanced hybrid AC-DC microgrids to maximize the loadability at the DGs' buses. The developed supervisory controller utilizes an OPF algorithm to minimize the microgrid load shedding in islanded operation mode. Numerical results verified the success of this method for maximizing the loadability at generation buses. However, the study case in this reference is a type two of hybrid AC-DC microgrids, which is operated in islanding mode. In other words, the class one of hybrid microgrids, with several independent DC buses and microgrids in gridconnected mode, has not been studied in this reference. The voltage profile enhancement, along with load sharing among DGs through a supervisory control scheme, has been considered in [111]-[112]. As developed and verified in [111], compensation of unbalanced voltage and harmonics in the system is possible through a hierarchical control scheme using multiple current loop damping strategies in islanded microgrids. In [112], a novel control scheme is proposed for islanded microgrids with several dispatchable DGs to improve the performance of the microgrid by adequately compensating the negative-sequence currents of the unbalanced loads.

However, none of the mentioned references considers the operation of the unbalanced microgrid in its grid-connected mode, where the droop control could not be utilized for sharing power among ICs. In [113], droop-controlled ICs are used to extend the autonomous power sharing among DGs in both $\mathrm{AC}$ and DC systems and manage the power 
flow among different $\mathrm{AC}$ and DC resources. But, the optimal operation of this system under an unbalanced load condition has not been investigated in this research. The coordination between DGs operation in both islanded and grid-connected modes of microgrid operation has been proposed in [114], where a complementary microgrid central controller is used to deploy secondary and tertiary control layers for DGs. This method is aimed to achieve seamless transitions between two operating modes using the cooperation of voltage and current and voltage-controlled voltage sources inverters.

In [115], the voltage unbalance been compensated in an islanded microgrid using a virtual output impedance method by measuring the negative-sequence voltage and current of DERs to find the voltage reference and tune the constant gain of closed-loop control. In [116], a robust control strategy for a grid-connected microgrid under an unbalanced load condition was introduced using an adaptive Lyapunov control mechanism to mitigate the negative sequence current due to unbalanced load conditions. A control scheme for unbalanced grid-connected microgrids was developed in [117], which is based on the correction strategy to guarantee the voltage balance at the PCC by compensating the negative-sequence loads' currents.

In [118], authors have proposed a supervisory control scheme for ICs aiming at increasing the microgrid's loadability at the PCC in grid-connected mode. The proposed method sets the active and reactive power bias factors for all three-phase ICs in such a way that it keeps the power balance at the PCC. However, it does not optimize the system for this power balancing and devotes the power imbalances to different phases of the ICs based on their capacities. Besides keeping the power balance at the PCC, numerical results in this reference demonstrate that the Voltage Deviation(VD) at different busses are improved, 
and grid losses are decreased due to keeping the power balance at the PCC. In [119], a twostep hierarchical power routing scheme for ICs in unbalanced hybrid AC-DC microgrids was developed; in the first step, the optimization tries to find an OPR within ICs to minimize the power losses in the system. In the second step, the proposed algorithm in [118] is used to keep the power balance at the PCC. However, the proposed hierarchical structure minimizes the objective functions sequentially, not using a multi-objective optimization algorithm.

In this dissertation, an effort was conducted to enhance the existing techniques for the optimal operation of unbalanced hybrid AC-DC microgrids in the grid-connected operating mode. This includes the development of a multi-objective optimization model to minimize the PIF at the PCC, active power losses and voltage deviation indices in a microgrid along with all operational constraints. In this model, the power routing is not only between three phases of each three-phase ICs, but also it is between different ICs in the microgrid. It means that the developed scheme considers the load shifting between ICs during the optimization process.

\subsection{Critical communications in modern power systems}

In addition to power market design and ancillary service studies in electricity markets, we conducted and completed a research project related to critical communication in modern power systems, its security and applications in modern smart grids. The Substation Automation System (SAS) plays a vital role in the optimal and reliable operation of modern power systems. The SAS is used for automating the control systems of substations and developing the remote monitoring and control mechanisms in energy industry [120]. Furthermore, it defines protocols for critical communications among IEDs, which should 
be fast, reliable, and secure enough to address the power system operation, control, optimization, and protection issues. These all bring a new level of challenges to the power system control and protection.

Generally, communication protocols and data model for data exchange among the IEDs are defined through substation automation standards. Although a variety of standards, protocols, and technologies have been developed in this regard, many of them are vendor dependent and they do not properly address the interoperability issues in complex systems, and do not support high speed communication technologies (e.g., Ethernet). Among all these standards, IEC 60870, MODBUS, and DNP3 are still being used in industry [121]. The IEC 61850 standard is the newest one which tries to address the above-mentioned drawbacks through new data models and protocols [122].

The IEC TC57 developed the IEC 60870 for basic remote-control communications between remote units and master stations and defined the profiles for different vendors to address the compatibility issues [123]. On the basis of the OSI model [124], which categorizes the communication network into seven layers, this standard mainly acts on the data link layer and uses RS-232, RS-485, or fiber optic interfaces at the physical layer for point to point network topology communications. Nowadays, the MODBUS protocol is used widely in industrial applications because it supports different network technologies such as serial communications, optical/radio networks, RS-232, RS-485, and RS-422. The MODBUS was basically developed for response/answer manner and acts at different layers of the OSI model [125]. The MODBUS is optimal protocol for serial communications and originally was developed for master-slave communication manner which means that it inherently is not suitable for Ethernet communications. Although the MODBUS plus 
covered the internetworking remote communications over the internet by adopting the TCP/IP protocol, this protocol still suffers from some disadvantages. For example, the sequence of events can be missed due to a lack of time stamping action. The distributed network protocol (DNP3) is usually used within the SCADA systems and defines the rules for interconnecting the automation systems [126]. Considering the OSI model, the DNP3 is a Layer-2 protocol with related addressing mechanism, link control, multiplexing, etc. Although the DNP3 is highly used in power industry applications, its main drawback occurs in the interconnection with data units and substation transport events, where the DNP3 protocol data packets lose their logical context.

In response to the above-mentioned standard limits, the IEC 61850 standard was developed to implement the modern networking technologies, data model, and communication protocols. This is a comprehensive standard with advanced protocols for device integration, data encapsulation, and network services, and therefore it provides a highly flexible design environment for each project and considers the communication technologies, object definition, and requirements. For example, there are three defined data models in this standard for different applications, i.e., GOOSE, SMV, and MMS. The GOOSE stands for the generic object-oriented substation event and is used for critical, time-sensitive, and multicast applications such as tripping/blocking signals within the wall of a substation. This type of communication occurs over the data layer (Layer 2) of the OSI model and must be completed in less than 4 milliseconds (ms). The SMV and MMS data models are used for sampled measured values and manufacturing message specification, respectively. In addition to communications over the data layer (horizontal communications), the routable communication protocols (vertical communications), such 
as R-GOOSE, for internetworking communications over the network layer (IP layer) has been defined by IEC 61850-90-5. This communication is also time sensitive and should be highly reliable and completed over a $10 \mathrm{~ms}$ time span including the network topology convergence. However, communication over the network layer using the IP deals with many limits because they were not built for these types of communications which are highly sensitive in terms of reliability, delivery order, and communication latency.

The R-GOOSE message, along with its applications in distribution automation, were described in detail in [127], where authors demonstrated the difference between GOOSE and R-GOOSE message and their data models and features. However, a proper communication middleware was not introduced, and network issues were not addressed. The applications of DDS in smart grids were evaluated in [128]. For example, DDS was used to improve smart grids communications by addressing the interoperability between different protocols such as DNP3 and MODBUS. However, it did not consider, and address interoperability issued related to the IEC 61850 and routable communications.

To route a GOOSE message over the WAN, adaptors are needed for configuration and interface with various equipment which use their own communication protocols such as Modbus, DNP3, IEC 61850, etc. For this purpose, by considering the formats which are described by data profiles, adaptors convert data and send them over MQTT, DDS, or AMQP communication protocols [129]. In this reference, the GOOSE message is translated to the OPC by the user agent to be transmitted over the WAN. However, this reference did not map the GOOSE data model over a DDS data object. In [130], a mechanism was introduced to deploy the IEC 61850 routable sample values and routable GOOSE messages for reliable data transfer of PMUs over the WAN to facilitate the 
communication for the WAMPAC. But the application of a broker-less communication middleware for fast and reliable data delivery was not presented. In [131], the DDS was deployed as a communication backbone for SAS. The process of mapping different communication traffics (GOOSE, SMV) into DDS data object were described properly.

However, network issues, routing service, and end-to-end delay for routable communication over the WAN were not addressed. For a successful routable communication over the WAN that meets the maximum acceptable latency, network traffic management is needed. Usually, tunneling techniques could help to manage traffics between networks. Nowadays, by creating the 5G networks, a specific slice can be allocated for delay sensitive messages and, for example, slice isolation can help with managing the traffic as mentioned in [132]. This reference categorizes the different message types used in distribution grid protection and control from the standard defined by IEC 61850 and maps onto the three major groups of $5 \mathrm{G}$ use cases.

It is worth mentioning that the $5 \mathrm{G}$ can address the end-to-end delay challenge in the substation-to-substation communication, however utilizing DDS in addition to the $5 \mathrm{G}$ can get the maximum benefit of the 5G network. DDS provides low latency scalable communication middleware, in addition to flexible and extensive QoS profiles which can be applied to individual data types instead of applying QoS to the whole stream or protocol in addition to QoS profiles, DDS binary encoding, and data filtering reduce the network bandwidth. Mapping GOOSE messages to DDS provides a solution that can be deployed using current technology and migrates it easily to the $5 \mathrm{G}$ when it becomes available. Moreover, due to possible cyber-attacks, the communication network should be isolated properly. One solution is to use the VPN for encryption and isolating data. However, to 
overcome the routable communication requirements, the network enhancement through special technologies is necessary. In other words, we are going to address this challenge for IEC 61850 routable communications by introducing a data-centric communication framework for R-GOOSE messages using the data distribution service standard. The proposed solution is a fast and reliable framework that covers all the IEC 61850 routable communication requirements along with the feature of multicasting, which could enable us to develop advanced protection schemes for modern power systems. Furthermore, we developed an experimental setup to measure the real-time end-to-end delay of R-GOOSE message communication over the network layer to verify the effectiveness of the proposed framework.

\subsection{Security of critical communications}

After developing a proper SS2SS communication framework, deploying effective security algorithm is necessary; however, using authentication techniques recommended by IEC 61850 , such as RSA, including message signing and verification, is infeasible because they are too slow (around $20 \mathrm{~ms}$ ) for this application [133]. As defined in IEC 61850 standard, the maximum allowed time delay to exchange a message over the IP layer is ten milliseconds based on the IEC 61850-90-5 technical report [134]. This report also provides communication protocols for R-GOOSE along with cyber-security protocols over WAN.

"EC TR 61850-90-5 security mechanism for R-GOOSE has three options:

1) None;

2) Signature (i.e. Authentication);

3) Signature and encryption. 
IEC TR 61850-90-5 security specifies the use of a signature using symmetric keys being applied to create a secure HMAC. The application messages are carried over an IEC TR 61850-90-5 session layer, which provides security and management via the 90-5 specific Group Domain of Interpretation (GDOI) protocol. GDOI support for 61850 protocols is described in the updated revision of IEC 62351-9, and the key exchanges use Group Domain of Interpretation (RFC 6407 - GDOI). Therefore, the objective of this research is to cover the security of the new applications of the IEC 61850 R-GOOSE messages for communication over the WAN. Since we developed a DDS-based communication framework for R-GOOSE messages, here at first, we try to deploy the DDS security algorithm on this platform and evaluate the security and latency of this algorithm. Afterward, we will propose the SSHA, which is independent of a communication middleware. In other words, the SSHA can be used by any communication middleware that is fast enough to route the message over the WAN.

\subsection{Advanced protection in modern power systems}

In this dissertation, the application of secure DDS-based communication framework for multicast R-GOOSE messages over the wide-area network for effective substation-tosubstation and substation to control center communications is represented by developing an advanced protection scheme to deal with extreme fault currents in modern power systems. In other words, we are going to show how secure routable communications can enhance the power system reliability and decrease the protection costs.

\subsubsection{High fault currents in power systems}

In modern power systems, the generation capacity is increasing significantly for responding to high electric energy demands. This generation expansion directly affects the 
level of short circuit in transmission systems and increases the fault current magnitude, which may pass the breaking capacity of circuit breakers. In another hand, the power system protection is one of the most critical and inseparable part of power system operation. An optimal protection scheme removes the fault current from the system in shortest possible time with minimum outage of power system equipment [135]. Regarding the system requirement, different protection functions may be deployed for each system as main and backup protections. For example, in transmission system usually main protection is the distance protection while in distribution system the overcurrent protection is chosen as the main protection [136]. Beside the protection scheme, short circuit level plays a vital role in selecting the protection equipment such as CBs. Generally, there are some standard CBs with different breaking capacities for each voltage level of the system, which are chosen based on the maximum SHC level in the system. For instance, in $220 \mathrm{kV}$ systems, two standard breaking capacities already exist in the market; 40 and $50 \mathrm{kA}$ [137]. For the systems with SHC level less than $40 \mathrm{kA}$ in worst fault condition, the CB with $40 \mathrm{kA}$ as BC is selected while for higher SHC levels (obviously less than $50 \mathrm{kA}$ ), the $50 \mathrm{kA}$ circuit breaker is chosen.

The problem arises when the SHC level goose beyond the standard limits for CBs. For example, in a $220 \mathrm{kV}$ system, if the SHC level is more than $50 \mathrm{kA}$, the standard CBs can't break the fault current which may cause the operation of backup protection with higher FCT and as a result, more equipment outages. One of the main reasons that $\mathrm{SHC}$ is growing up is the generation expansion planning due to high electric energy demands in power systems. Moreover, in recent years, the consumption of electric energy has been increased significantly. As an example, in USA the total electricity demand has been increased from 
0.35 billion $\mathrm{kWh} / \mathrm{year}$ in 1949 to 4 billion $\mathrm{kWh} / \mathrm{year}$ in 2017 [138]. It means that the power system has also been expanded and more generation capacities have been installed in the system. Furthermore, the increasing trend is observed, and we will expect more electricity demand in future years. From protection point of view, the more generation capacity means higher SHC level in the system. Hence, it is very important to limit the SHC level and keep it below the breaking capacity of CBs in the system for reliable protection action against fault currents. For this purpose, several solutions have been proposed and deployed such as network reconfiguration or installing different types of (FCLs in the power system including high impedance transformers, current limiting reactors or superconductor FCLs [139]. All these methods have some advantages and disadvantages, however, most of them add a new device to the system, which is usually very expensive and needs high maintenance. In this dissertation, we are going to introduce a method based on intelligent switching actions which removes the need of installing FCLs in the system.

\subsubsection{Communication capabilities in the power system protection}

In recent years, by developing and implementing the IEC 61850 standard in substation automation and advanced power system protection, there are some potentials to develop intelligent protection schemes using defined protocols in IEC 61850. This standard is known as an international standard for IEDs' communication in an electrical substation using defined protocols such as MMS, SMV and GOOSE. As defined in [140], these data models can be transmitted within a substation through a LAN or for substation-tosubstation communication by a WAN. These potentials and communications' capabilities have been partially used in distribution systems and for microgrid protection [141]. To the best of authors' knowledge, the capabilities of IEC 61850 communication protocols have 
not been used for designing an intelligent protection scheme to deal with extreme fault currents in HV transmission systems where distance protection is deployed as main and backup protections.

Therefore, in this dissertation, an intelligent protection scheme is introduced based on cascading switching actions within and between substations to manage very high fault currents in transmission systems. This protection scheme uses communication capabilities defined in IEC 61850 to design smart cascading switching actions to isolate the faulty part of the system and acts as a supervisory protection scheme which monitors the fault current in the system and makes real-time decisions to deal with extreme SHC currents when conventional protection scheme cannot clear the fault in shortest possible time. The proposed method uses the standard logical nodes of the standard to develop a new agent for each substation which can communicate with all IEDs within the substations and also IEDs in neighborhood substations. After developing this agent, simulation results are reported in detail to show the effectiveness of proposed method in presence of very high fault currents in transmission systems. In addition, the results are compared with conventional distance protection and ideal protection schemes, when FCLs are deployed, from different points of view including FCT, rotor angle stability margin, number of switching actions and equipment outages to demonstrate the advantages of this method in smart power systems.

\subsection{Problem statements}

Nowadays, the structure of the electric energy system has changed due to the high penetration of DESSs, RESs, EVs, and the creation of the operation of MGs. These changes, along with deregulation in electricity markets, have made many challenges and 
problems for energy policymakers as well as power system operators. From the regulatory point of view, in the power systems with independent MGs and significant uncertainties due to new energy resources and energy storage, a very well-organized competitive energy market structure which optimally mitigates any power mismatch through a fast optimization method is needed. To the best of our knowledge, the literature lacks a framework that is fast enough for real-time market operation of a multi-microgrid system. Moreover, proper communication infrastructure has not been studied for data exchange in a multi-microgrid power market. Therefore, in this dissertation, a competitive hierarchical electricity market framework along with sits communication infrastructure were developed and verified to address all above-mentioned issues.

Additionally, by developing the $\mathrm{BC}$ technology as a secure $\mathrm{P} 2 \mathrm{P}$ trading platform, there are many issues to implement this technology in electricity markets. However, blockchains are still an emerging topic and technology with numerous regulatory and technical challenges. For example, the scalability and complexity of the BC in the energy market, privacy of market players, and operational security of power systems are significant challenges in this filed. All the issues mentioned above must be addressed in order to implement and the $\mathrm{BC}$ technology for power market transactions. In this dissertation, we developed and verified a BC-based energy market for inter-microgrid energy transactions considering the power system constraints for energy trades over transmission lines.

In addition to energy markets in modern power systems, ancillary services should also be developed well to guarantee the reliable operation of the power system under deregulated power markets. One of the main challenges is to optimally mitigate the congestion of power transmission lines in real-time operation of power systems through a 
well-developed market based ancillary service. For this service to be optimal and costeffective, it should deploy all dynamic capacity of transmission lines, and the algorithm should be converted to a feasible solution in real-time operation. Hence, an adaptive RTCM algorithm was developed and verifies to optimally mitigate the congestion from power systems.

Furthermore, unbalancing operation of microgrids causes operational and power quality problems for optimal operation of microgrids and power systems especially in gridconnected mode. Therefore, in this dissertation unbalanced loading is addressed by developing and verifying a multi-objective optimization model within an unbalanced hybrid AC-DC microgrid. In addition to power market design and ancillary service studies in electricity markets, we conducted and completed a research project related to critical communication in modern power systems, its security and applications in modern smart grids. The problem arises from time limit defined by IEC 61850 for routable GOOSE messages for SS2SS communication and its security measure. The standard defines the end-to-end delay for R-GOOSE message less than $10 \mathrm{~ms}$, therefore, an effective communication is needed to meet this requirement. Furthermore, the security of this framework should be fast end effective enough to protect these critical messages from possible cyber-attacks. In this dissertation, a DDS-based communication framework is developed and verified for R-GOOSE messages along with proper security algorithms that makes it possible to complete the secure SS2SS communication within its time limit.

\subsection{Research objectives}

In this dissertation, the concept of the electricity market for a multi-microgrid system is presented. The idea of the multi-agent-based, hierarchical, and fully competitive 
electricity market for multi-microgrid systems is to consider the optimal operation of autonomous MGs and facilitate the inter-MG energy transactions. The main goal is to minimize the dependency of the area to utility support and avoid the high real-time energy price served by the utility network. Detailed analysis of market players should be considered during the design of the electricity market. On the other hand, the market should be fully competitive and give this opportunity to the participants to optimize the ire benefits. For an electricity market to be reliable and optimal, secure data exchange among market agents within the system is critical.

For this purpose, at first, proper communication protocols must be selected to address the communication needs regarding the structure of the market. Afterward, the security of this communication framework should be addressed appropriately. Once the market design is achieved, the developed framework will improve the operation of multi-microgrid areas and reduce external network support. The optimal structure of the market reduces the energy price for market players and consequently improves social welfare. Therefore, a game-theoretic based energy transaction framework is developed to accurately represent a fully competitive energy auction for trading between energy buyers and sellers.

Moreover, the communication platform is built for secure data exchange between different agents in the system. This communication framework was developed based on the DDS as middleware that makes it possible to run different market levels separately over different topics within a DDS domain. The second research objective is achieved by implementing the $\mathrm{BC}$ technology in the electricity markets for secure $\mathrm{P} 2 \mathrm{P}$ energy transactions. The main goal of the BC-based energy market is to use a distributed ledger for energy transactions, improve market security, and reduce system vulnerability. For this 
purpose, a hierarchical BC-based power market was developed in this dissertation for multi-microgrid systems. This structure provides a distributed energy trading framework within each MG and a BC-based inter microgrid market considering the power system security constraints and customer privacy.

Real-time energy market ancillary services such as congestion management and optimal operation of unbalanced hybrid AC-DC microgrids are discussed in this dissertation. An adaptive RTCM is developed and verified to demonstrate the capabilities of existing transmission line in reducing RTCM costs. In addition to this, t multi-objective optimization model for optimal power routing between and within ICs is developed and verified to optimally mange unbalanced system loading at the PCC while reducing active power losses and improving the voltage deviation indices.

Another objective of this dissertation is to develop and verify an effective and secure communication framework to address the needs for critical R-GOOSE communication for SS2SS data exchange for protection and control mechanisms. A lightweight security algorithm is developed to address the cybersecurity of critical communications in a smart grid along with the application of this secure communication in advanced protection schemes are developed.

\subsection{Original contributions of this dissertation}

The original contribution of this desertion is to develop electricity market frameworks for the optimal and secure operation of a system containing multi microgrid systems. This goal is achieved by a comprehensive study of electricity market requirements to cover energy transactions within and between MGs in a power system. The purpose of this study is to understand and address significant issues for microgrids' electricity markets, including 
market model, scalability, accessibility, competition feature, energy balance mechanism, the security of the electricity market data, and communication infrastructure. This study resulted in designing optimal and secure frameworks for multi-microgrid systems with intelligent market agents through the following steps:

- We developed a multi-agent-based hierarchical market framework containing three levels of the markets to optimize energy transactions in a multi-microgrid system using a distributed optimization model.

- These three levels are the Day-Ahead, Hour-Ahead and Real-time Markets, respectively.

- In the Day-Ahead Market (DAM), we developed a game-theoretic-based double-auction model which makes the market more competitive and consequently decreases the energy price.

- For the Hour-Ahead Market (HAM), we developed and an optimization model to minimize the energy mismatches with minimum cost.

- We developed a reverse auction model for the Real-Time Market (RTM), where energy resources compete to feed the remained demand with lower price.

- We developed a fast, scalable, reliable and secure communication framework for the developed market structure in a multi-microgrid system using DDS as a data-centric communications middleware based on the real-time publishsubscribe protocol.

- We verified the proposed market model by implementing this market model on the modified 37-bus IEEE distribution test feeder system, including three MGs. 
- The results indicated that the developed market framework decreases the energy cost, increases the system resiliency in a deregulated environment.

The security of the transaction framework for power market is another aspect of the research in this dissertation. The robustness of modern power systems depends on the level of cybersecurity against cyber-attacks. Since energy transactions could significantly affect the power system operation, these transactions should be evaluated through a secure network to enhance the power system reliability. Our contribution in this area is as below.

- In this dissertation, we evaluated the security level of different energy transaction frameworks by calculating the probability of the Power Market Failure (PMF) due to cyber-attacks for centralized, decentralized and distributed energy markets.

- We developed a mathematical model for each energy transaction framework in order to obtain numerical results and compare them.

- The numerical results demonstrated that the distributed energy transaction framework is the most reliable system against cyber-attacks comparing with traditional centralized and modified decentralized energy transaction frameworks.

The distributed energy transaction framework uses a distributed leger through the BC technology. However, power system constraints and feasibility of energy transactions should be evaluated before confirming it. Therefore, in this dissertation we developed a hierarchical BC-based energy transaction framework for multi microgrid systems. Our contributions in this area are listed as below. 
- We developed a hierarchical BC energy market containing several market levels.

- We developed the Microgrid layer, where local blockchain are used for energy transactions within each microgrids. The network layer is the second layer of this framework which deploys another BC for inter-microgrid transactions.

- We developed an equivalent microgrid model to summarize local ledger and makes inter-microgrid transactions possible in the network layer.

- We verified this hierarchical BC-based transaction platform in an area with three MGs and compared the results with conventional $\mathrm{BC}$ which do not consider power system constraints before approving a transaction in the system.

In addition to above mentioned contributions and research, we completed research related to optimal operation of unbalanced hybrid AC-DC microgrids with following contributions.

- We developed a multi-objective optimization model aimed to minimize the PIF at the PCC, active power losses and voltage deviation indices within a microgrid considering all operational constraints.

- In this model, we used the power routing not only between three phases of each three-phase ICs, but also between different ICs in the microgrid.

- We verified this model through simulation and experimental results at the FIU smart grid testbed.

The reliable operation of the power system with deregulated power markets highly depends on the ancillary services to guarantee a high quality of service to all power system entities. In this dissertation, we developed an adaptive RTCM algorithm considering the 
thermal adaption of transmission lines to manage the congestion optimally. Our original contributions in this study are as below.

- We developed a real-time optimization model to manage the congestion in smart power systems.

- The developed an adaptive RTCM algorithm which uses the dynamic thermal rating of transmission lines during a congestion in the system to decrease the congestion cost an minimize the load shedding and generation rescheduling.

- We developed a hybrid optimization model as a powerful tool for the RTCM optimization problem, including an AANN and a modified PSO algorithm.

- We verified the proposed RTCM algorithm by simulation results and compared it with the conventional RTCM algorithms. The comparison demonstrated that the developed algorithm is fast enough and cost-effective to be implement in real-time congestion cases.

Besides all the works mentioned above, another aspect of this dissertation is related to a research project about the cybersecurity of the IEC 61850 as a power system automation standard. It contains deep research on the security and vulnerability issues for routable communications using in this standard to detect the cybersecurity needs. Our contributions in this research are as below.

- We developed and a communication platform for R-GOOSE messages using the DDS as communication middleware.

- We verified and this platform by developing and measuring actual data from an experimental setup at the FIU smart grid testbed. 
- We deployed the DDS security algorithm to the developed platform to add a security layer to GOOSE data over the network.

- We also developed a lightweight security algorithm for fast communication over the network, like R-GOOSE messages, which could be implemented by any communication middleware.

- Finally, we developed an intelligent switching mechanism based on the IEC 61850 communication protocols to protect the power systems against extreme fault currents and remove the necessity of installing FCLs in power systems.

\subsection{Dissertation organization}

This dissertation is organized in twelve chapters, including this chapter, which contains an introduction to the dissertation research topic, a literature review of subjects under study along with problem statements, research objectives, and original contributions.

In Chapter 2, the structure of microgrids' power markets are described, different types of trading mechanisms, deregulation of power markets, and new topologies of energy trading are introduced for modern power systems.

In Chapter 3, the security of electricity markets is evaluated. At first, the concept of power system security is compared with energy market security. Therefore, the ideas of secure operation of power systems under different constraints are discussed. Afterward, general structures of power markets are introduced, and a mathematic model is developed to compare the security levels of the most well-known electricity market frameworks and categorize them regarding their robustness against possible cyber-attacks.

Chapter 4 presents a multi-agent-based decentralized electricity market for multimicrogrid systems. This structure proposes a hierarchical three-level energy trading 
platform to run a decentralized energy market in an area with several independent MGs. A game theory model is developed for a competitive double-auction based energy market along with a bidding strategy for market players. The market will propose an optimization model for the second level of clearing and uses a reveres auction model for real-time energy markets.

Chapter 5 demonstrates the communication framework for market transactions introduced in Chapter 4. It starts with introducing the data-centric communication framework and Data Distribution Service as a communication middleware and continues with presenting a DDS-based platform for data exchange between market agents. This platform is then optimized to reduce the number of required topics in each DDS domain. This chapter ends with introducing the security measure for the DDS systems and implementing it to the developed optimal communication platform for the market operation of the system.

In Chapter 6, a new model of the electric energy market is developed based on the $\mathrm{BC}$ technology. In this model, the concept of a hierarchical BC-based energy market is introduced, which is the combination of decentralized and distributed energy markets. The local BCs, network BCs, and summarized ledger concepts are presented. This model defines an equivalent model of MGs for inter-MGs transactions using the BC technology. The main advantage of this model is to consider the power system constraints before verifying transactions that need to used transmission systems.

Chapter 7 is devoted to the optimal operation of unbalanced hybrid AC-DC MGs. This could be considered as an ancillary service for MGs which need to connect to the utility grid. This service is provided mostly by Interlinking Converters within the MG, where an 
optimization algorithm to control the active and reactive power flow for each phase of these converters. Load shifting between different substations is modeled by an optimal distribution of EVs as a mobile load in the system using an effective pricing mechanism. Then a comprehensive multi-objective optimization model is presented along with experimental results obtained from FIU smart grid testbed to validate the developed algorithm.

In Chapter 8, an essential ancillary service in power markets is introduced to manage the power transmission congestion in the real-time operation of the power system. This service is called the RTCM, which calculates and uses the quasi-dynamic thermal rating of transmission line through a hybrid optimization algorithm to adaptively mitigate the congestion in a power transmission system with minim cost. Rescheduling the energy resources along with demand response are the tools for this service, which can include independent MGs in different points of the system with capabilities to participate in this ancillary service market.

Chapter 9 explains substation automation standards and introduces critical communication for modern power systems. It continues by focusing on the IEC 61850 standard and evaluating the GOOSE and R-GOOSE protocols. Subsequently, a DDS-based platform is introduced for R-GOOSE messages for substation-to-substation communication. The effectiveness of this platform is then verified by hardware-based experimental results at the FIU smart grid testbed.

Chapter 10 addresses the development and verification of security issues related to the R-GOOSE communication platform developed in Chapter 9. At first, security challenges are explained. Afterward, two security mechanisms are developed: the first one is based on 
the DDS middleware, and the second one is based on a new technology to protect critical communications over the WAN. Any proper communication middleware can implement this technology such as message centric or data-centric.

In Chapter 11, an important application of a secure R-GOOSE framework developed in Chapters 9 and 10 is developed and verified for the protection purpose of modern power systems. In this advanced protection scheme, an intelligent switching scheme is developed and deployed to protect the power systems with extreme fault currents higher than the breaking capacity of circuit breakers. The successful implementation of this algorithm, which is based on cascading switching actions, will result in enhancing the security of the power system against high fault currents, improving the stability and removing the need for installing the FCLs or replacing the circuit breakers.

Chapter 12 is the conclusion. It summarizes the dissertation outcomes, highlights the research significances, and finally recommends some research topics for future works. 


\section{Chapter 2 Microgrids' Participation in Power Markets}

In the restructured power markets, due to competition between service providers, the better quality of service at a lower price compared to the monopoly market structure is expected. From the power system point of view, Microgrids are aggregated controllable loads and generators. Based on the order 888 issued by the FERC, open access, and nondiscriminatory power market should be available for all participants. Moreover, an OASIS should be developed based on the order 889 to facilitate reforming the power market models [12].

\subsection{Power market models}

Generally, there are three major power market models; Pool model, Bilateral contract, and Hybrid model. Each model provides a different type of competition and market structure.

\subsubsection{Pool model}

The pool model is a kind of centralized market with an independent market operator clearing the market. In this model, buyers and sellers participate in the market by submitting their bids and the amount of energy they want to buy/sell. The market operator collects all requests from participants, rearranges them in increasing order for sellers, and decreasing order for buyers to find the MCP regarding the last accepted offer. The Pool model contains two different types as below.

1. Double auction model

In this model, both suppliers and demands are competing to win the auction, as shown in Figure 2.1. The intersection of both demand and supply curves indicates the Market 


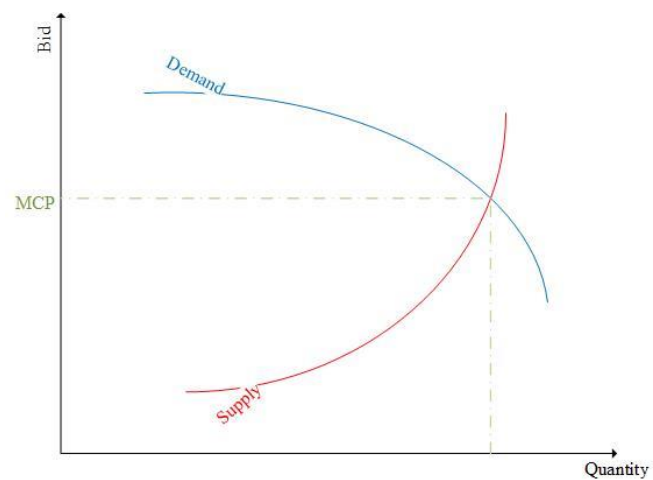

Figure 2.1: The double auction model
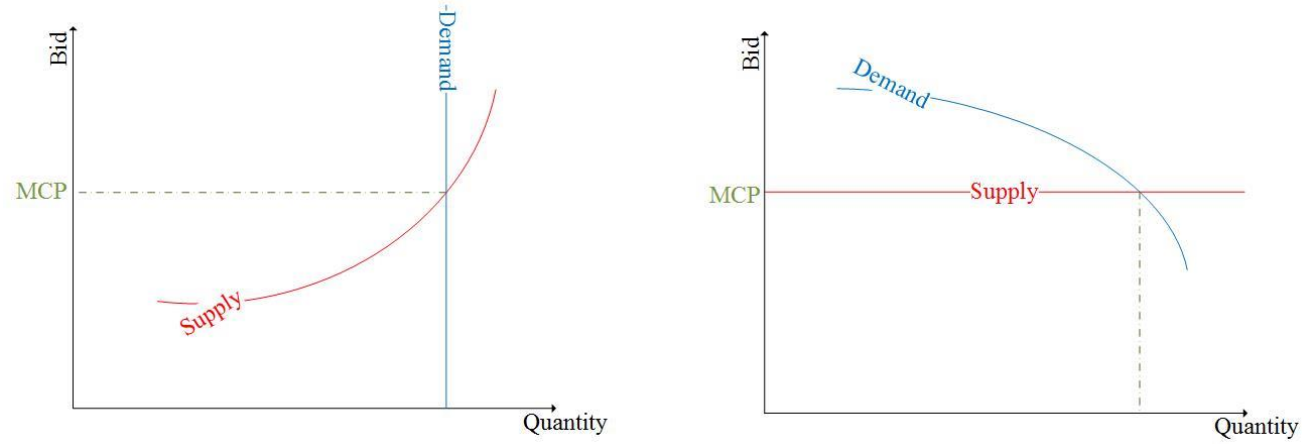

Figure 2.2: The reverse auction model

Clearing Price (MCP). Any participant on the left side of this point is a winner of this auction. Losers, who are on the right side of this point need to adjust their energy or sell/buy it in other markets.

\section{Reverse auction model}

Other types of Pool market are called reverse auction models, where for example, a load aggregator can represent the forecasted load by the system operator, and the competition could be between generators to feed this load by minimum price. It can also happen for loads to compete and send their bids to be the winner of using a certain amount of energy provided by aggregated generators. These two types of Pool model are called reverse auction model as shown in Figure 2.2.

In a Pool market, the market can be settled based on the MCP or based on the price bids, which is called pay as bid. In the first scheme, all participants are charged/paid based 
on the MCP, while in the pay as bid strategy, all winners in the pool market are charged/paid as their price bid. The competition in the pool market and its dynamic feature usually result in the marginal cost for energy price because considering any more benefit margin for market players may cause them to lose the market.

\subsubsection{Bilateral contract model}

In this model, buyers and sellers directly trade the energy and negotiate in the market with no need to use the energy pool. The bilateral contracts contain the energy price, quantity, the point of energy generation, and consumption ion the network. The ISO is then evaluating the feasibility of the transaction to ensure the reliability of the service and manage the power transmission congestions. If a transaction is not approved by the ISO, both parties should find another seller/buyer, which may cause a higher energy price for them or changing the load pattern/generation pattern. The power transmission system and distribution network play a vital role in the approval of a transaction in the system. Therefore, there should be clear rules, pricing methods, and non-discriminatory access to these systems for all market participants. Transmission and distribution companies are responsible for building and maintenance of power corridors in the system and are paid by the system user and wholesale suppliers.

\subsubsection{Hybrid model}

The Hybrid model is a combination of the Pool and Bilateral contract models. This model enhances the process of energy balancing in the system. Participants can trade the energy through the PX by bilateral contracts. Other available generations and loads are then entered into the Pool market for an economic dispatch based on energy and demand cost functions. 


\subsection{Microgrids in competitive power markets}

The competitive power market models motivate small customers (e.g., end-users) and distributed generators to not participating in the wholesale market individually. Instead of it, they can deploy aggregators to actively participate in the market on their behalf to maximize their benefits and minimize their costs. The need to use aggregators raise from this fact that for a retail market operator, it is not feasible to manage too many individual participants. On the other side, an aggregator needs an excellent communication platform to handle a massive amount of data exchange between entities and make proper decisions to participate in the market per collected supply/demand data. Since the MGs are considered as aggregated controllable loads with local generations, they can efficiently participate in power markets.

\subsubsection{Participation in the retail market}

The role of ISO in the wholesale market is clear and accepted by the market players. However, the competitive power market is extending to the distribution level and retail market, where consumers can liberally choose their service provider. MGs, as aggregated loads with local generation capability, are considered as a retailer that should address all technical and regulatory challenges for a retail market aiming at reducing the energy cost. MGs can participate in open competitive retail markets to sell/buy power and various ancillary services to/from other market participants. This participation needs advanced metering and billing mechanisms along with comprehensive information of customer profiles within the MG. Besides, to participate in the retail market and do energy transactions with other retail and service providers, MGs need to establish internal MG electricity markets for energy trades between sellers and buyers within the MG. It is 
because of this fact that nowadays, independent participants within MGs want to participate in the electricity market actively. For example, a resident with an installed PV panel might want to sell its extra energy to a neighbor locally [95]. Therefore, an open-access and wellregulated infrastructure is required within each MG mainly when MG is operating in islanding mode.

\subsubsection{Participation in ancillary services}

Besides active participation in retail markets, $\mathrm{MG}$ can contribute to the ancillary service markets such as voltage/reactive power support, frequency control, demand response, reliability, and stability services. It is based on their potentials and capabilities, the type of DGs and DERs, the capacity of their energy storage and load flexibilities in reacting to the service requirements. For example, if the MG is equipped with fast DG with high ramp rates to follow the load changes in the area, it can actively participate in the frequency control program. It can also happen if there is enough energy storage within the MG linked to fast-responding converters. MGs also can provide reactive power support for local voltage control services by injecting/absorbing reactive power when it is needed. All these services depend on the structure of the MG, the EMS as and control capabilities.

\subsection{Multi-Microgrid Power Markets}

A multi-microgrid system contains clusters of microgrids within an area of the power distribution network [142]. These microgrids are physically connected using one or several feeders in the distribution network as shown in Figure 2.3. As mentioned before, a microgrid is considered as an aggregated and controllable load from the power system point of view. In contrast, a multi-microgrid system can present the integration of many RESs. 


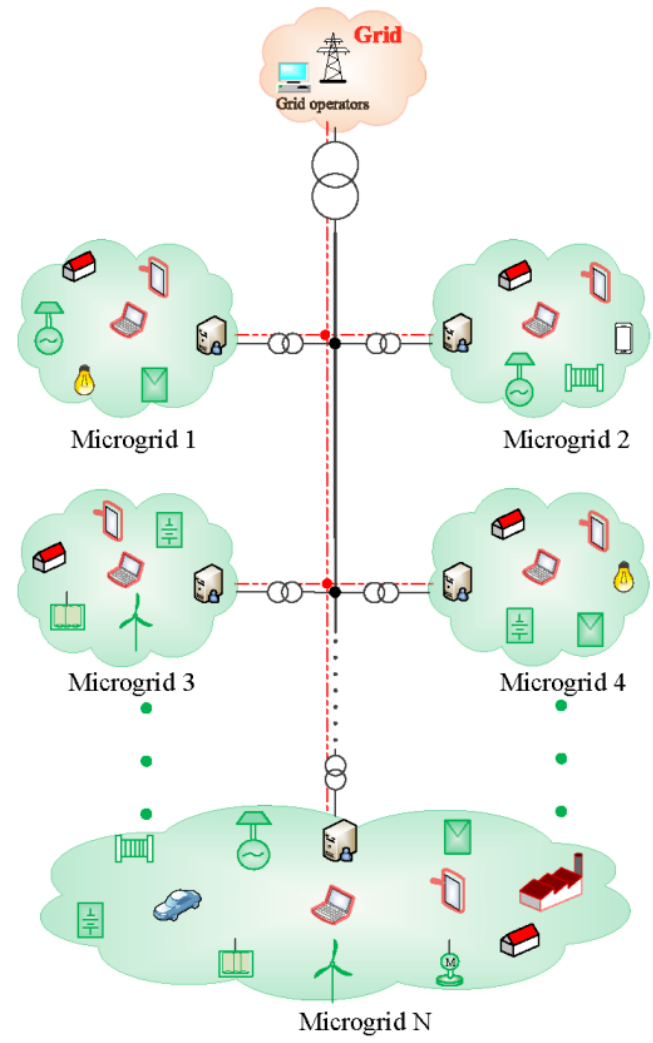

Figure 2.3: The concept of multi-microgrid [143]

Therefore, a multi-microgrid system can also operate as a reliable energy source to support the power system during any major contingency or to enhance the economic operation of the power grid. There are different technical and financial challenges which must be addressed before practically deploying this capability of a multi-microgrid system as are described in this section.

\subsubsection{Technical challenges}

In a multi-microgrid system, a significant challenge is the optimal operation of individual microgrids as well as coordinated operation of all microgrids in that area [144]. The priority of each independent microgrid is to keep its internal energy balance and feed all loads with a high quality of service. For this aim, and due to rapid changes in the energy balance of each microgrid, a multi-microgrid system needs to check the overall energy 
balance of the area and the necessity of using the utility grid support or supporting the utility grid by injecting power to it. This is all possible by developing and deploying an effective energy management system within the area by an extensive communication infrastructure as well as smart metering devices. The load forecasting and following programs, uncertainties of RESs, different types of DGs, and unbalanced operation of microgrids are the main technical challenges for a multi-microgrid system. For example, in [145], an optimum strategy for energy management of microgrids was introduced considering the DGs, DESSs, EVs and demand responses, and in [146], and energy management scheme was developed based on the contingencies to support the power network.

\subsubsection{Market challenges}

For a multi-microgrid system with one control center, all calculations, energy estimations, operation coordination, and interacting with the external grid are possible much more accessible than a multi-microgrid system with independent microgrids and control centers. It is because of this fact that each microgrid in this system is autonomous, can be disconnected from the network, and work in islanding mode or connect to the system and exchange energy with other microgrids or the utility network. Treading energy between microgrids needs a well-organized market platform to facilitate energy transactions and enhance the reliability of the whole area by reducing the dependency on the external grid. Furthermore, it should provide open access, competitive and non-discriminatory energy market to guarantee less energy cost and high quality of service. In addition, by increasing the number of microgrids within an area, the willingness for energy trade with pother microgrids might increase, and the distribution feeders might be congested. Therefore, for 
inter-microgrid transactions, power system operational constraints must be evaluated before approving any energy trade between participants. It is necessary for the secure operation of the power system, especially in peak load conditions. This means that independent operation and energy exchange must be under the supervision of a local independent system operator and local market agent. The wholesale energy market does not need the data of inter-microgrid transactions, and it just requires to be updated about energy exchange with the utility grid. Hence, local markets with private rules might be created and deployed in different areas.

There are many studies in the literature in this regard. For example, in [147], and energy trading scheme was developed based on the Nash bargaining theory aimed to encourage microgrids for energy trading. In [148], a multi-objective optimization model was introduced for energy trading between microgrids and the power grid. A distributed energy management technique was developed in [149] to minimize the operating cost of the multimicrogrid system by energy trading between microgrids. The improvement of power dispatch efficiency through decoupling the economic dispatch in the multi-microgrid system was proposed in [150].

In addition to that, a two-step optimization algorithm was presented in [151] to minimize the operating cost of energy transactions between microgrids. The application of cooperative game theory in multi-microgrid power markets was introduced in [152] considering the benefits of microgrids' owners, system operators, and generation cost reduction. In another research, a game theory approach was developed for energy balance in a multi-microgrid system [153]. 


\subsubsection{Ancillary service challenges}

In traditional monopoly power markets, most of the ancillary services were provided by big generators in the system, which can result in more energy costs and losses to deliver these services. In deregulated electricity markets, ancillary services are provided by all market players based on their capabilities and through several separate markets.

In the wholesale electricity market level, ancillary services are defined and provided through different markets besides the primary energy market. However, in the retail market level, the participation of microgrids in the ancillary service markets is highly useful for more reliable operation of the power system as well as reducing the cost of ancillary services in the system. The main challenge of this participation is the lack of wellestablished retail markets for ancillary services in multi-microgrid systems. For example, MGs need the motivation to participate in different ancillary services and get comparative benefits from the market. Therefore, once the multi-microgrid energy transaction framework is designed, efficient, and well-organized ancillary service markets also must be developed to get benefits of microgrids' participation. This contribution can decrease the energy loss over the transmission and distribution systems due to the location of a microgrid which is usually in the distribution level and very close to most consumers.

Furthermore, due to the competition for ancillary services, and lower energy cost of DERs in MGs comparing with big utility generators, the cost of ancillary services is reduced significantly. There are some efforts in the literature to establish ancillary service for multi-microgrid systems. For example, a coordinated strategy for managing the RESs in multi-microgrid systems was developed for voltage support and frequency regulation using a heuristic algorithm [154]. However, an effective pricing mechanism for 
autonomous participation of independent MGs is still needed for fully competitive and open access energy markets in multi-microgrid areas. 


\section{Chapter 3 Electricity Market Security Assessment}

The security term has a different meaning in energy security, the security of power systems operation, and power market security. The concept of energy security must be cleared at first; then, the secure operation of the power system is described following by defining the security of power markets. These concepts are highly dependent, and failure in each one directly affects others. For example, if the security of energy resources is not guaranteed, generation units cannot produce electric energy, and consequently, the power system cannot operate securely by losing energy resources. Failure in the secure operation of the power system is linked to the electricity markets. Lack of energy resources and reliable market participants could cause market collapse and energy mismatch in the system. Therefore, in this chapter, concepts of energy security and secure power system operation are briefly described. Afterward, the safety of electricity markets in terms of cyberattacks is evaluated for three significant structures of power systems, including centralized, decentralized, and distributed markets. Since the distributed electricity market points the blockchain technology, this technology is also described to show its market structure in order to evaluate and compare its security level with other market structures. Finally, mathematical models for these three market platforms are developed and numerically compared to categorize the electricity market frameworks from the security point of view.

\subsection{Energy security}

The energy security topic has several dimensions; The short- and long-term security as well as the environment and national security [155]. 
- The short-term security is for a response to the immediate need for electric energy aimed to minimize the partial or overall blackout likelihoods. For example, in peak load hours, expensive spinning reserves generation units must be available to follow the load pattern.

- The long-term security is to address the increasing load demand by expanding the generation capacity and transmission system. The power system expansion planning is not only a technical challenge; there are many political and policy factors that must be considered in this regard. For example, the location of new generation units, the possibility to install new transmission lines considering the environmental limits, country, and state limits, etc.

- Environmental security profoundly affects electricity systems. It is because of this fact that still most electric energy is generated by burning fossil fuels, coal, and natural gas, which consequently much $\mathrm{CO} 2$ production. On the other side, global climate change due to increased $\mathrm{CO} 2$ in the atmosphere is a major problem. Therefore, the power plants are an easy target for politicians and governments to control the emission. Thus, generation expansion planning for long-term energy security impose additional challenges to the investors and power market players.

- National security is related to the external enemies' threats, such as military/terrorist threats. The power transmission system is a critical component of power systems which its failure can significantly threats national security. Therefore, the operation and protection of this system remain regulated and are not affected by electricity markets to avoid the direct effect on national security. 


\subsection{Power system operation security}

The energy security could guarantee the available electric energy resources for shortand long-term as well as proper transmission system capacity. However, the secure operation of the power system is essential for the security of the power system and delivers the energy to end-users. It contains numerous technical challenges, including securityconstrained unit commitment and economic dispatch of available energy resources. Furthermore, in modern power systems with high penetration of renewable energy resources, their secure dispatch is critical. In addition to dispatching the energy resources, many control action and optimization algorithms are needed for the secure operation of power systems. For example, providing necessary tools and control scheme for reactive power support to maintain the voltage magnitude within standard limits, frequency control schemes, transmission congestion management methods, load shedding, protection coordination, etc.

\subsection{Electricity market security}

Generally, electricity markets enhance energy security and power system operation security [156]. It is because of competition among market players to gent more benefits from the market by more investment for energy generation and providing different ancillary services for the energy systems. It could remove the market power and enhance the security of power systems. It is worth mentioning that private companies will conduct researches to improve the efficiency of their generation units to minimize their cost to be able to compete in the electricity market by their marginal price. It will also result in less pollution by better filtrating due to the penalty for $\mathrm{CO} 2$ production. As mentioned above, the electricity market inherently boosts electric system security. However, the electricity 
market must be secure to provide a reliable transaction platform for market players and omits any threats to the power market. Otherwise, the security of the power system is seriously threatened, and blackouts are probable. For example, in 2015 Ukraine blackout was due to data vulnerability and malicious events or California blackout in 2000 and 2001 was due to market data manipulation $[155,157]$ and $[157]$.

The security of the power market depends on the structure of the energy transaction framework and communication system. User authentication and its activities in the power market must be evaluated before approving any energy transaction in the power market. Furthermore, the feasibility of each transaction within the system should be verified by the ISO, considering the operational limits and availability of the energy resources. Additionally, the power market must be secure enough against possible cyber-attacks, which is highly dependent on the market structure and the security of communication infrastructure.

In the rest of this Chapter, the main structures of power markets are presented and compared together to categorize the security level of each market framework, and we will discuss the security of communication infrastructure in Chapter 5.

\subsection{Electricity market structures}

We described the three primary electricity market structures in [158]. These are Centralized, Decentralized, and Distribute markets, as shown in Figure 3.1. In each case, market players trade energy through a different framework with some advantages and disadvantages. It should be noted that in addition to these main platforms, there are some other market structures, which are combinations of these leading platforms. 


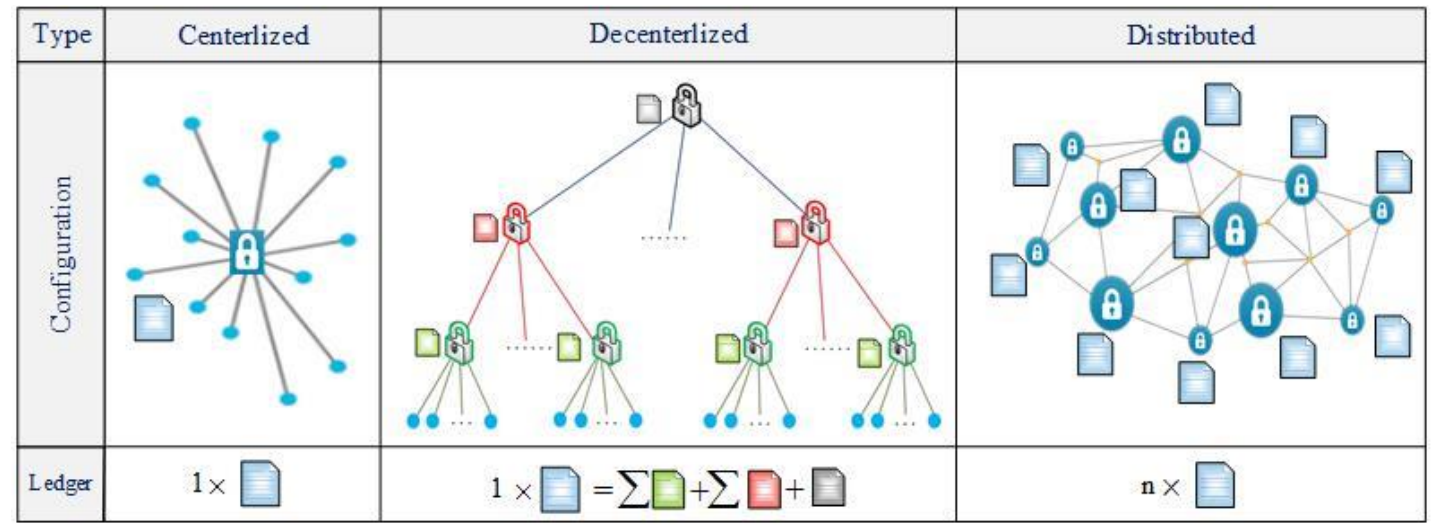

Figure 3.1: Electricity market structures and communication links [158]

\subsubsection{Centralized electricity market}

The centralized market is a well-known market structure that has been traditionally used for almost all financial transactions and non-financial trades. In this platform, there is a trusted third-party that collects all data, analyses them, and run the market, and any transaction between network entities is done through this agent.

This type of market is easy to use because all members should be registered by the trusted third-party and afterward, by receiving their certificates and security keys, can participate in the market based on the market rules. In this structure, all kinds of energy transactions are possible; for example, bilateral trades could happen between participants or market players could actively participate in auction-based energy markets. Moreover, by developing and deploying advanced data analytic methods and parallel computing techniques, the market agent can evaluate many transactions, simultaneously and speed up the market-clearing process.

However, the main weakness and drawback of this market are that the centralized market suffers from the single point of failure feature. The reason is that data are just saved in the central market agent over one ledger. This ledger consists of all transaction data as well as the system information. Therefore, by a successful attack to the central point of the 
market, the attacker can get access to all players' data in detail, which could make market failure and consequently, energy system collapse.

Besides, by a notable increase in the number of market participants, not only the length of the ledger is increasing significantly, but also the market-clearing process and transaction feasibility assessment might be slow down. It could happen in modern power systems with too many individual participants. Nowadays, investors of renewable energy resources would like to participate in the power market actively and sell their energy in open access markets. Furthermore, distributed generators, autonomous microgrids, and even electric vehicles are interested in having access to a secure, reliable, and open access energy Market. As a result, the number of market players is increasing significantly. On the other side, real-time energy markets with high market-clearing speed are needed to mitigate the uncertainties of modern power systems due to the high penetration of RESs. Therefore, in addition to off-line, the day ahead and hour ahead energy markets, there is a need for real-time markets which can run very close to operation time.

\subsubsection{Decentralized electricity market}

A centralized market can address the drawbacks mentioned above for the centralized mar. This market divides the system into several sub-systems and tries to clear the market, locally. The hierarchical structure of this market makes it possible to handle many transactions in proper processing time, increase the data security by breaking the ledger among different agents and address the weakness of the single point of failure effectively. However, in this market, individual participation of market players is affected by higherlevel agents, which act as aggregators and market agents. In other words, peer-to-peer transactions are just possible in a specific part of the system if the higher-level agents 
provide the platform for this kind of trades. A comprehensive decentralized electricity market along with its communication platform will be introduced in Chapters 4 and 5, respectively.

\subsubsection{Distributed electricity market}

The fully distributed market framework uses the BC technology and synchronized ledgers. This technology is widely used for virtual currency trades such as Bitcoins and similar cryptocurrencies. In this technology, there is no trusted third party to evaluate transactions, running the auction, or act as an aggregator for individual participants. In other words, each market player could actively trade with other participants. All agents provide the validity of transactions in the system, which have access to all previous transactions in the system through their highly secure and synchronized ledgers.

Generally, if the BC technology is used, all participants have a copy of all previous transaction records. For any set of new transactions between participants, all the transaction data are simultaneously broadcasted to all participants using a specific encrypting method. Receivers would verify the originality of data, check the possibility of a transaction through a voting mechanism, and pack data as a block. This block is then added to the existing blocks using a highly secure encrypting mechanism.

\subsubsection{Sending, receiving and verifying transaction data}

As described before, each market agent has a copy of all previous transactions in a blockchain format. Furthermore, it should participate in validating new transactions in the system. For this purpose, each agent needs to have a public and a private key. The private key is utilized to create a specific encrypted signature to submit data to other agents. All

receivers need the sender's public key to decrypt its signature and confirm the accuracy of 
the received data. Therefore, each agent needs the public key of all other agents in the network. This process has been described in detail in [159].

After confirming the accuracy of received data, agents start validating the feasibility of received transaction considering the pervious chain of transaction records in the ledger. A voting mechanism is used to confirm the validity of each transaction as follow.

$$
N_{a}>N_{r}
$$

Where $\mathrm{Na}$ and $\mathrm{Nr}$ are the number of agents who approved and rejected the transaction, respectively, for a transaction to be accepted, at least $51 \%$ of active agent must approve that transaction as shown by (3-1). By evaluating and validating a set of transactions using protocols mentioned above, a data block is built and signed to add to the blockchain ledger.

\subsubsection{Generating transaction block and ledger synchronization}

A blockchain includes time-based serial blocks which are cryptographically linked together. Each block contains a pack of approved transactions. Hash-based cryptography is deployed to add a new package of approved transactions to the existing blockchain. Hash algorithms are used to create a message digest from input data [98]. The output message digest from a typical hash function (e.g., SHA-256) has a 32-bit word including $0,1 \ldots 9$, and $A, B \ldots F[160]$. It should be noted that the hash function has specific characteristics. For example, it is practically impossible to invert this message digest. It means that we can't find the input data from output data when the hash function is used to encrypt data. Furthermore, the hash function is unique for input data. It means that it is impossible to find two different input data that create the same output from the hash function. The input data not only includes the transaction data but also contains block number, timestamp, date, the hash of the previous block, and a random number. 


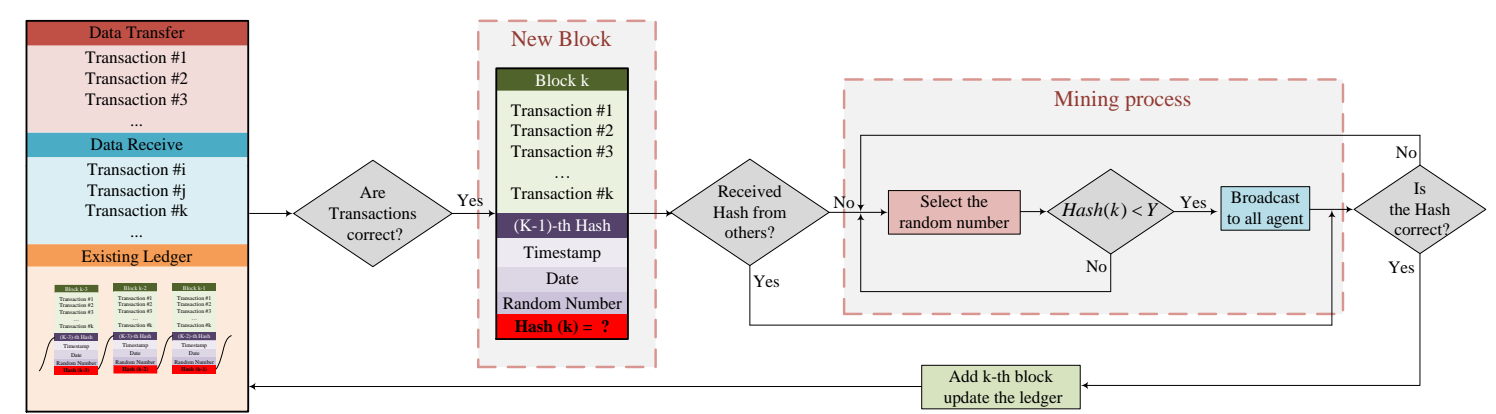

Figure 3.2: The BC algorithm to build and process a new block by processing the transactions' validity and the mining process.

This random number should be determined in such a way that the output data the from hash function meet some constraints as defined for the BC and shown by (3-2).

$$
\operatorname{Hash}\left(X_{i}\right)<Y
$$

Where $\mathrm{Xi}$ includes $\mathrm{i}^{\text {th }}$ input data to the hash function, and $\mathrm{Y}$ is a specific format for the hash output that we define for the blockchain. The process of finding an appropriate random number to meet the constraint (2) is called the mining process, and those agents who try to solve this problem are called miners. It should be noted that some or all participants can compete together to find the solution for this problem, but the mining process requires a high computational power to find the solution, especially when the constraint is more limited. In this mechanism, the first miner who solves the problem is awarded, and the solution is sent to other agents for confirmation. When the solution is approved by a minimum number of agents, a new block can be added to the existing data chain. At this point, it is impossible to change the data on the blockchain as they are linked through a highly secure cryptography mechanism.

The blockchain flowchart for a miner who solves the puzzle is described in Figure 3.2. It includes data verification, creating a new block, the mining process and adding a new block to the existing blockchain ledger. 


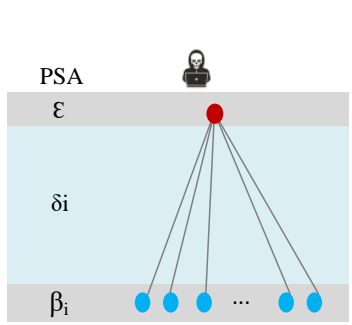

(a)

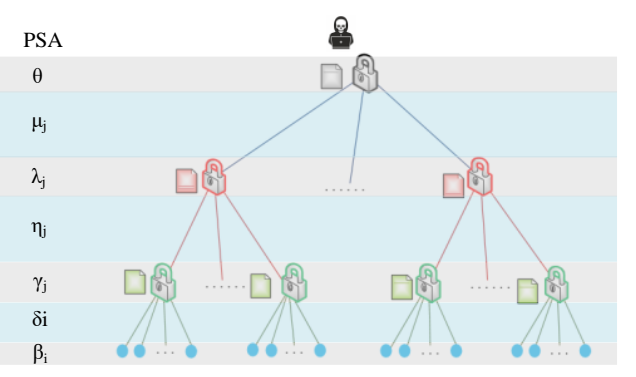

(b)

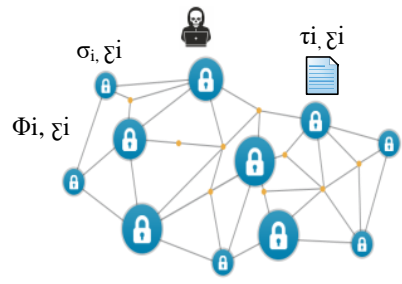

( c )

Figure 3.3: The PSA for different sections and communication links of three market platforms

\subsection{Adversary and threat model}

In order to compare the security level of the three market structures, we consider an adversary model by assuming that all market entities are fully trusted. However, external enemies attack the system by spoofing the market agents and communication network to obtain sensitive data. Afterward, they inject bad data to the system for available energy quantity and price bids, which can launch collusion attacks aiming at falsely accusing legitimate market players of double spending in the energy market.

Therefore, to evaluate the robustness of each market structure against external cyberattacks, we consider a PSA for each market entirety as well as each communication channel and evaluate the PMF due to successful attacks to a different number of agents in the system.

\subsection{PMF modeling}

In this section, the PMF is calculated for three market structures, which are attacked by the same eternal enemy aimed to collapse the market and consequently, the power system [158]. For this purpose, a mathematical model for the PMF of each market structure is developed, then three models are numerically compared under some assumption. 


\subsubsection{PMF model for centralized marketplace}

As shown in Figure 3.1, in a centralized marketplace, there is a central market operator, and only one ledger, which collects all transaction data, verifies them, and settles the market. All market agents should directly communicate with the market operator through their own communication links. Therefore, cyber-attacks may occur on the market agents before they send data, the communication system during data transfer, or the market operator after receiving and processing. Therefore, the PSA for each above-mentioned section of this market framework is shown in Figure 3.3a by $\beta_{i}, \delta_{i}$ and $\varepsilon$, respectively. The PMF of this market is shown by $\mathrm{P}_{0}$ and calculate by (3-3) based on the total probability theorem.

$$
P_{0}=\left(\frac{1}{4} \prod_{i=1}^{n} \beta_{i}\right)+\left(\frac{1}{4} \prod_{i=1}^{n} \delta_{i}\right)+\left(\frac{2}{4} \varepsilon\right)
$$

Where $\mathrm{n}$ is the minimum number of attacked market agents for a successful market failure. $P_{0}$ is consists of the PMFs for each part of this market platform.

\subsubsection{PMF model for decentralized marketplace}

The decentralized market contains four layers, including market players, aggregators, local market agents, and general market agents. It also has hierarchical communication links between different layers. Therefore, cyber-attacks are probable in all sections of this system by different PSA values, as shown in Figure 3.3b. Attackers may launch an attack on market players, communication links, or higher-level agents. For a successful market failure, we assume that bad data must be injected form at least several market players (n), aggregators $\left(\mathrm{n}_{1}\right)$ or local market agents $\left(\mathrm{n}_{2}\right)$. These numbers are denoted by $\mathrm{n}, \mathrm{n}_{1}$ and $\mathrm{n}_{2}$, respectively. 
Therefore, the PMF of this market platform is shown by $\mathrm{P}_{1}$ and calculate by (3-4).

$$
\begin{aligned}
P_{1}=\left(\frac{1}{8} \prod_{i=1}^{n} \beta_{i}\right) & +\left(\frac{1}{8} \prod_{i=1}^{n} \delta_{i}\right)+\left(\frac{1}{8} \prod_{j=1}^{n_{1}} \gamma_{j}\right)+\left(\frac{1}{8} \prod_{j=1}^{n_{1}} \eta_{j}\right)+\left(\frac{1}{8} \prod_{k=1}^{n_{2}} \lambda_{j}\right)+\left(\frac{1}{8} \prod_{k=1}^{n_{2}} \mu_{j}\right) \\
& +\left(\frac{2}{8} \theta\right)
\end{aligned}
$$

The total probability theorem is used for this modeling because attacking to each section of this platform is independent of other sections.

\subsubsection{PMF model for distributed marketplace}

In the blockchain-based market framework, all participants have a synchronized copy of the ledger the same as others. Regarding this structure and the BC protocols, launching a successful attack on this system needs more effort compared to centralized and decentralized platforms. Market agents, communication links, and the BC ledger might be attacked by the PSAs as $\sigma_{\mathrm{i}}, \varphi_{\mathrm{i}}$ and $\tau_{\mathrm{i}}$, respectively, as shown in Figure 3.3c. Furthermore, for an attack to be completed, the attacker must also obtain a pair of public and private keys for each agent. We show the PSA for this information by $\Sigma$ i. Therefore, the PMF of this platform is denoted by $\mathrm{P} 2$ and calculated as below.

$$
P_{2}=\frac{1}{3}\left(\prod_{i=1}^{n} \sigma_{i}\right) \times\left(\prod_{i=1}^{n} \xi_{i}\right)+\frac{1}{3}\left(\prod_{i=1}^{n_{3}} \varphi_{i}\right) \times\left(\prod_{i=1}^{n} \xi_{i}\right)+\frac{1}{3}\left(\prod_{i=1}^{n_{4}} \tau_{i}\right) \times\left(\prod_{i=1}^{n} \xi_{i}\right)
$$

Where $\mathrm{n}$ is the minimum number of market players which must be attacked to lunch a successful market failure. The minimum number of communication links and ledgers for probable market failure are $\mathrm{n}_{3}$ and $\mathrm{n}_{4}$ that are determined by (3-6) and (3-7), respectively.

$$
n_{3}>\left[\frac{N \times(N-1)}{4}\right]
$$




$$
n_{4}>\left[\frac{N}{2}\right]
$$

Where $\mathrm{N}$ is the total number of agents in $\mathrm{BC}, \mathrm{n}_{3}$ is calculated based on the voting mechanism, which defines at least $51 \%$ approvals from all agents to confirm a transaction. Therefore, for a successful attack, at least $51 \%$ of communication links should be spoofed and attacked successfully. The same logic is used to determine the minimum number of ledgers which must be attacked to fail the BC-based market successfully. It means that we need to alter the data in at least $51 \%$ of all market ledgers.

\subsection{Comparison models}

The three developed PMF models for electricity market structures contain many independent variables. Therefore, to compare the level of security of these frameworks against cyber-attacks, one way is to simplify these models by reducing the number of independent variables. For this aim, we can link the PSA values based on this fact that the PSA of higher-level and major agents in the market structure would be less than lowerlevel agents because more robust security algorithms are usually deployed for these agents. Consequently, for centralized, decentralized and distributed market frameworks we assume a series of variables relationships as shown by (3-8), (3-9) and (3-10), respectively.

$$
\begin{gathered}
\beta_{i}=\delta_{i}=10 \varepsilon=\alpha \\
\beta_{i}=\delta_{i}=5 \gamma_{j}=5 \eta_{j}=10 \lambda_{j}=10 \mu_{j}=20 \theta=\alpha \\
\sigma_{i}=\xi_{i}=\varphi_{i}=10 \tau_{i}=\alpha
\end{gathered}
$$

Where $\alpha$ is a probability variable that is used to simplify these models., for example, as we can see in (5), if the PSA of a market agent in the centralized platform is $\alpha$, it is assumed that the PSA the market operator is $\alpha / 10$. Hence, the models described by (3-3), (3-4) and 
(3-5) can be simplified as shown by (3-11), (3-12) and (3-13), respectively.

$$
\begin{gathered}
P_{0}=0.5 \times\left(\alpha^{n}+0.1 \alpha\right) \\
P_{1}=0.25 \times\left(\alpha^{n}+(0.2 \alpha)^{n_{1}}+(0.1 \alpha)^{n_{2}}+0.05 \alpha\right) \\
P_{2}=0.333 \times \alpha^{n} \times\left(\alpha^{n}+\alpha^{n_{3}}+(0.1 \alpha)^{n_{4}}\right)
\end{gathered}
$$

As can be seen in theses equations, the number of variables reduced, and numerical comparison is possible which, are presented in the next section.

\subsection{Numerical results}

The study case is an electricity market with 27 participants $\left(n^{\max }=N=27\right)$. If the decentralized framework is deployed, we assume nine aggregators $\left(n_{1}^{\max }=9\right)$ and three local market agents $\left(n_{2}^{\max }=3\right)$. Therefore, $n, n_{1}, n_{2}, n_{3}$ and $n_{4}$ have the following ranges:

$$
\begin{gathered}
1 \leq n \leq 27 \\
1 \leq n_{1} \leq 9 \\
1 \leq n_{2} \leq 3 \\
176 \leq n_{3} \leq 351 \\
14 \leq n_{4} \leq 27
\end{gathered}
$$

In this study, we set $n_{1}=3, n_{2}=1, n_{3}=176$ and $n_{4}=14$. By these values, the PMF models are just function of two variables; $n$ and $\alpha$. Therefore, by changing the values of these variables, we calculate and compare the PMF of three market structures. Figure 3.4 shows the values of centralized market PMF $\left(\mathrm{P}_{0}\right)$ for a different number of attacked agents (n) and continues values of $\alpha$ between 0 to 1 . The same calculations are presented for decentralized $\left(\mathrm{P}_{1}\right)$ and distributed $\left(\mathrm{P}_{2}\right)$ markets by Figures 3.5 and 3.6, respectively. The results demonstrate that in all three market frameworks, by increasing the minimum number of agents which are needed for successful market failure, the PMF is decreased. 


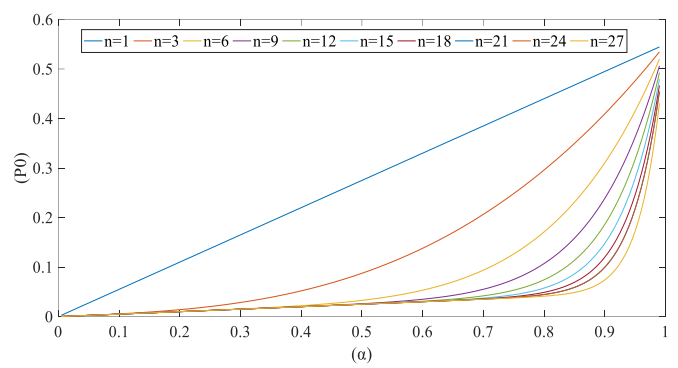

Figure 3.4: PMF of the centralized market

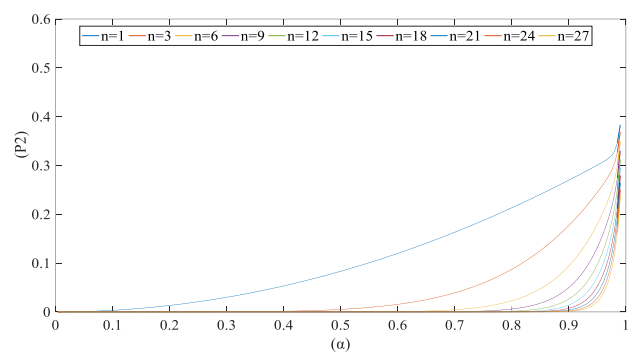

Figure 3.6: PMF of the distributed market

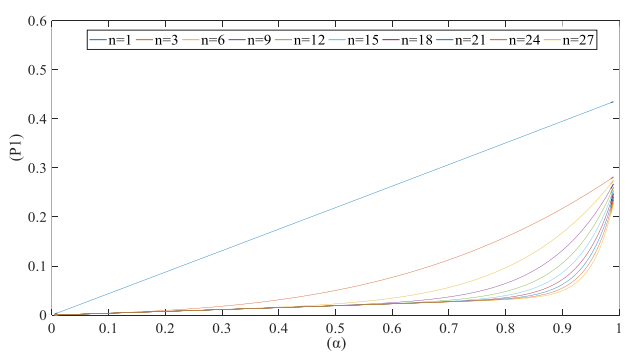

Figure 3.5: PMF of the decentralized market

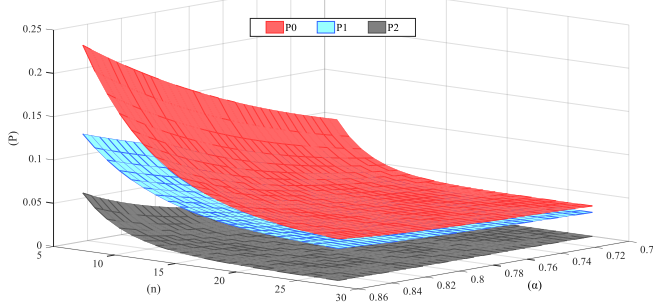

Figure 3.7: PMF of the three market frameworks

On the other hand, by increasing the value of $\alpha$, the PMF is increased for all $n$ numbers.

Figure 3.7 compares three PMF models in a 3D graph. Two vertical axes show the variables: $\alpha$ and $\mathrm{n}$, and the horizontal ax displays the PMF values for three structures. It is seen that for specific values of $\mathrm{n}$ and $\alpha$, the PMF of the distributed electricity market framework has the lowest value while the centralized framework has the biggest one. It means that from the cybersecurity point of view and robustness against cyber-attacks, we can categorize three market structure as follow:

1- Distributed structure

2- Decentralized structure

\section{3- Centralized structure}

Therefore, the structure of the electricity market can have an extreme effect on its cybersecurity. Distributed and decentralized electricity markets benefit from higher security levels because, for a successful attack on these systems, several security layers exist in the market frameworks. 


\section{Chapter 4 Optimal Decentralized Electricity Market for Multi-Microgrid}

\section{Systems}

In this Chapter, a multiagent-based energy market is developed for multi-microgrid systems using game-theoretic and hierarchical optimization approaches [161]. The developed method is tailored to achieve the optimal operation of smart microgrids in distribution systems. In fact, because of rapid load variations in distribution systems, it is necessary to develop fast optimization algorithms that minimize the power mismatch within and between microgrids. Therefore, a three-level market framework is developed for this purpose. The first level comprises a game-theoretic double auction mechanism for the day-ahead market, and the next two levels are optimal rescheduling and inter-microgrid reverse auction models for the hour-ahead and real-time markets, respectively. By using the hierarchical optimization algorithm in a multi-agent-based system, it is anticipated to minimize not only the optimization solution time but also reduce the dependency of the area on the network in grid-connected mode or load shedding in islanded mode. Furthermore, DR capabilities along with rescheduling of DESSs and DGs could be utilized in all market levels, which will lead to optimal operation of multi-microgrid systems. Agents were developed in DIgSILENT PowerFactory, and DDE is activated for communication among agents communicating through a DDS, which utilizes the RTPS communication protocol. The developed framework is applied to the modified 37-bus IEEE distribution test feeder system to validate the effectiveness of this market structure. 


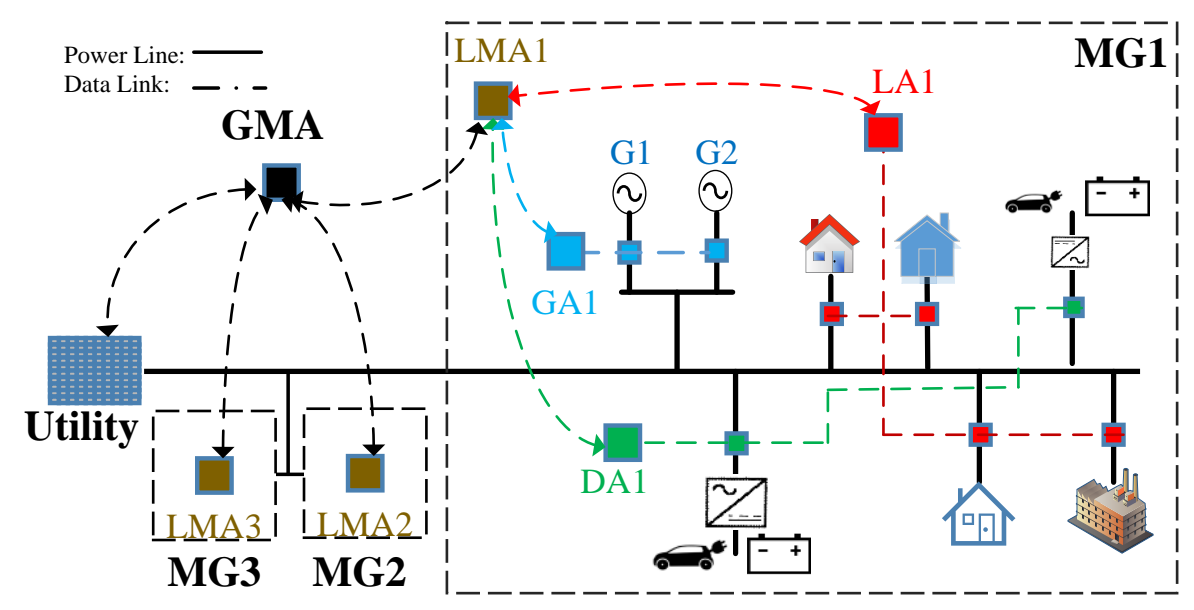

Figure 4.1: Architecture of a Multiagent-based multi-microgrid system.

\subsection{Architecture of the multi-microgrid system}

Figure 4.1 presents the architecture of the Multi agent-based system in a multimicrogrid structure. Each microgrid contains different types of loads, DGs, and DESSs, which are controlled and that actively participate in the electricity market by means of their individual EMSs considering their constraints, forecasting parameters, and optimal strategies. The LA, DA, and GA are used to gather and sort data from the individual loads, DESSs, and generators' EMSs, respectively. These agents communicate with their LMA to send and receive specific data during each market level. To run an optimal intermicrogrid market, LMAs are linked to the GMA, where all required data from all microgrids and the utility are collected and analyzed. The LA in this study is considered as a retailer which communicates with all the EMSs of all the individual microgrid loads' EMSs and participates in the market to provide the forecasted energy by minimum cost. 


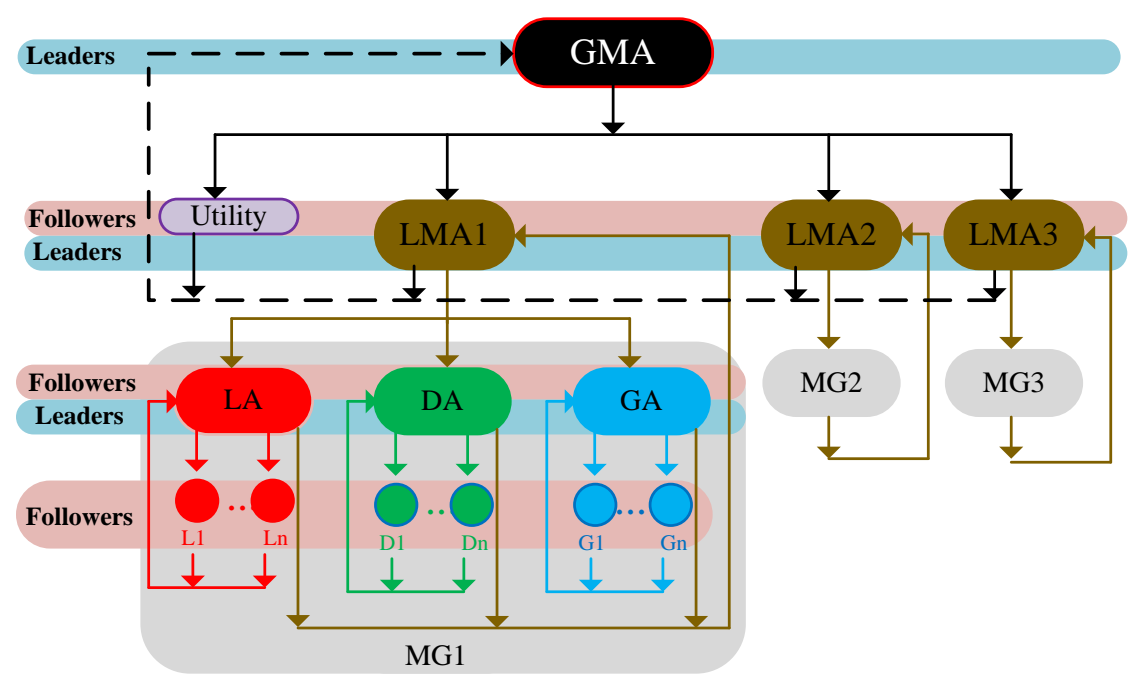

Figure 4.2: Hierarchical optimization framework for a multi-microgrid system with leaders and follower for three MGs, LMAs, Utility and GMA.

\subsection{Hierarchical optimization framework}

Hierarchical optimizations, also called multi-level programming techniques, are utilized to find the solution of decentralized scheduling problems in a hierarchical structure with several decision-makers [162]. Generally, a hierarchical organization includes interactive decision-making units where decisions are made sequentially from upper levels, called leaders, to lower levels, called followers. Each agent maximizes/minimizes its own benefits/costs individually, after processing the other agents' actions. The basic concept of the hierarchical optimization illustrates that leaders should define the objective functions and then ask followers to submit their individual optimal decisions for participation. Afterward, leaders optimize the overall system benefit and send their outputs to the followers' agents. This process is repeated until an optimal solution is reached. Therefore, this algorithm is highly appropriate for an actual multiagent-based open-access market, where each agent seeks its interest independently. 


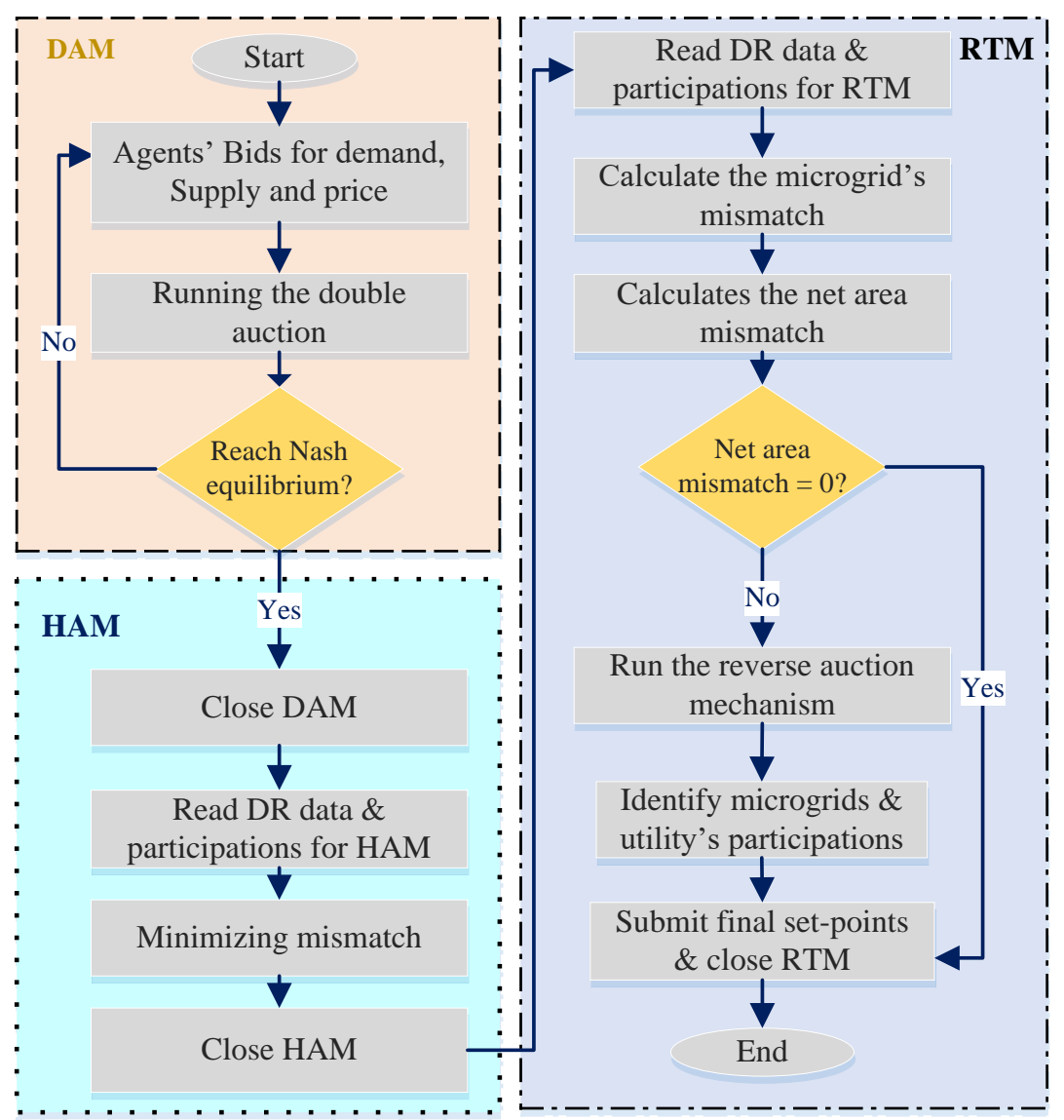

Figure 4.3: Proposed algorithm for the multi-level market operation including DAM, HAM and RTM.

Figure 4.2 shows the developed hierarchical optimization framework for this study, which transforms a massive optimization problem into smaller manageable pieces, which are easier to solve and allows for parallel implementations that will result in significantly lower solution time. In the developed structure for multi-microgrid operation, in each level of the market, EMSs (followers) send the data of the loads, DESSs, and generators to their leaders: LA, DA, and GA, respectively. These leaders, who are followers of an upper leader (LMA), send organized data to the LMA. The LMAs in all microgrids and the utility agent are all followers of a general leader (GMA). Consequently, LMAs and the utility agent send their analyzed data to the GMA when they are requested. 


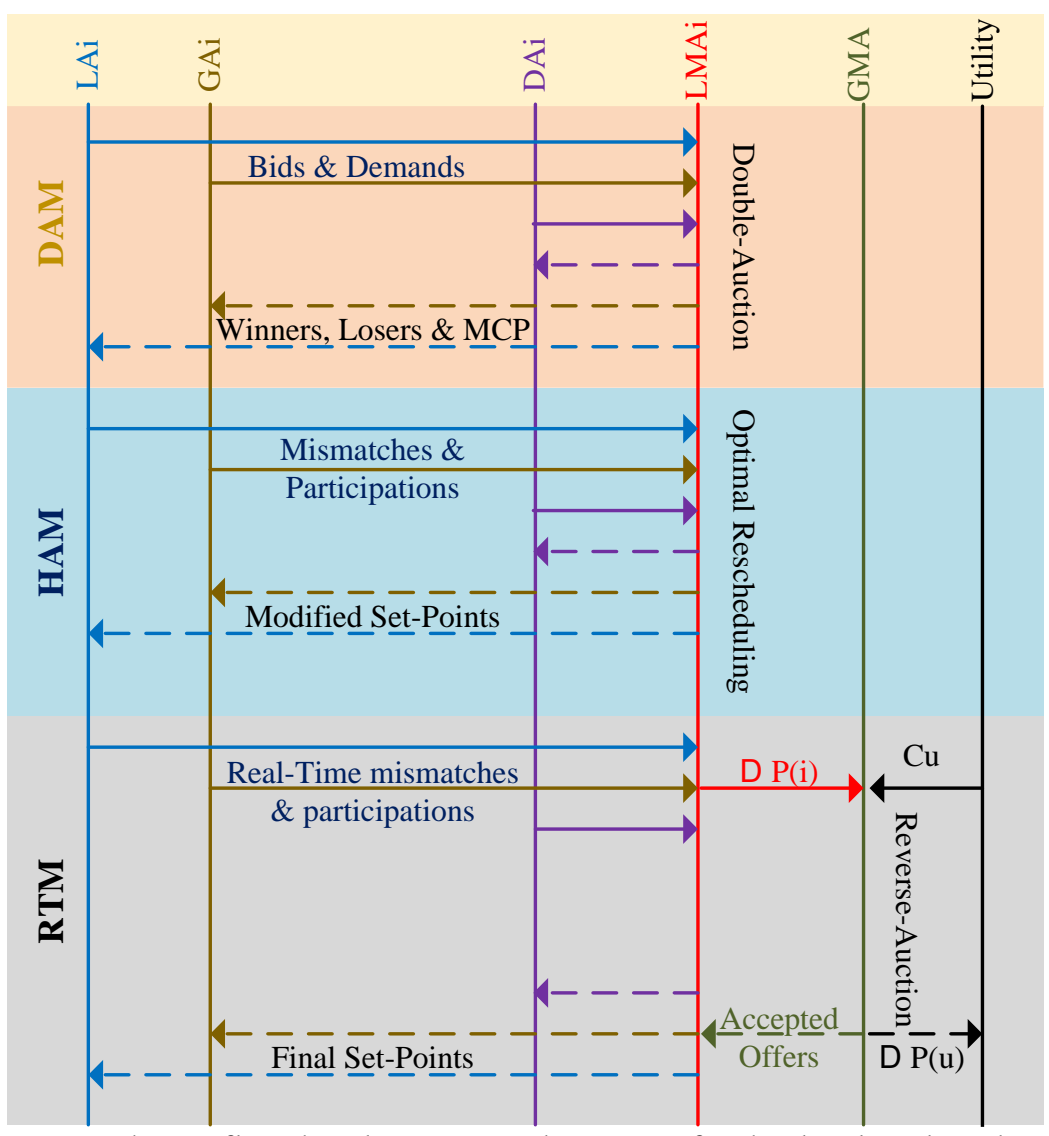

Figure 4.4: Data exchange flowchart between market agents for the developed market framework.

\subsection{Developed energy market framework}

Based on the proposed hierarchical optimization framework, a three-level market framework is developed aiming at providing an optimal open-access market where all market players participate independently. It is worth mentioning that each market level has its own hierarchical structure. In the first level, load agents (Loads and DESSs, which are in charge mode) try to buy their forecasted power by minimum price using a game-theoretic approach. In the next two levels, the market is run to mitigate the forecasted mismatches reported by the different agents, optimally. The purpose is to minimize the energy mismatch during operation to decrease the energy cost, dependency on the utility, and any mandatory load shedding. 


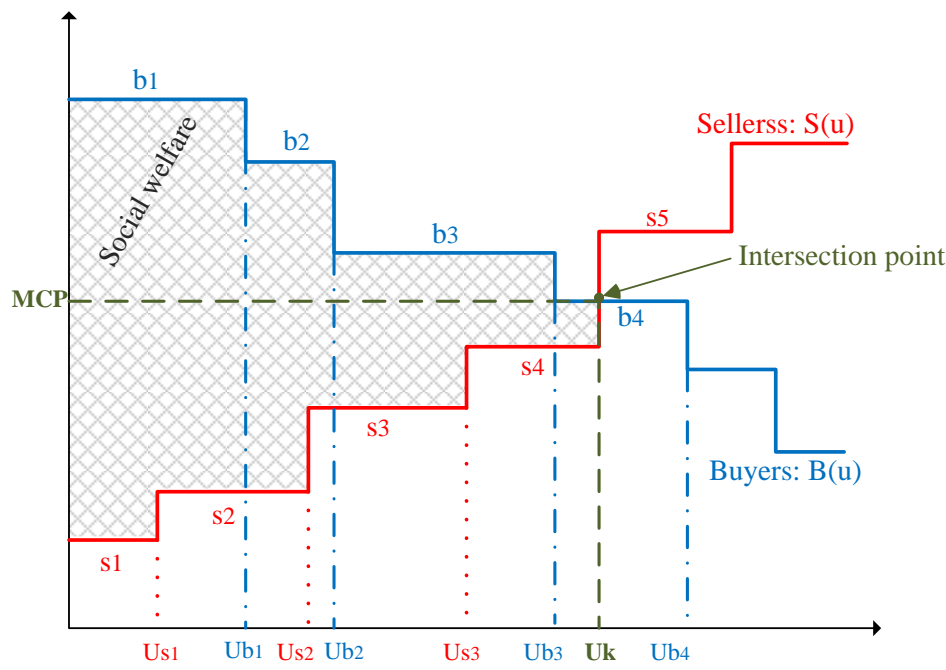

Figure 4.5: Double auction mechanism

Figure 4.3 illustrates the developed three-level market framework, and Figure 4.4 presents the data exchange flowchart for this process. A comprehensive description of each level is presented in following sub-sections.

\subsubsection{Level 1 - Day-Ahead Market}

In this level, the DAM, a double-auction mechanism which was presented in [163] is deployed. However, to create a more competitive market, a game-theoretic approach is developed for this double-auction model.

\subsubsection{Double auction mechanism}

In a double auction mechanism, buyers and sellers submit their bids and ask prices, respectively, to an auctioneer whose task is to clear the market. The auctioneer sorts the buyers' bids in decreasing order, and the sellers' asks in increasing order. In this paper, $\mathrm{B}(\mathrm{u})$ and $\mathrm{S}(\mathrm{u})$ are buyers (LAs and DESSs in charging mode) and sellers (GAs and DESSs in discharging mode) functions, respectively. By maximizing the social welfare (the difference between what buyers are bidding and what sellers are asking for, as labeled in Figure 4.5), the market-clearing process is as described below. 


$$
\max (\text { socialwelfare })=\max \int_{0}^{U_{k}}[B(u)-S(u)] d u
$$

The output of this optimization, including the MCPs and the accepted energy trades, are determined by the intersection point of the buyers' and sellers' curves, as shown in Figure 4.5. It is worth mentioning that technical issues such as control delay of DGs must be considered when the rescheduling is programmed for the next market interval. This is very important in islanding mode, when the microgrid should keep the balance between load and generation. To keep the frequency in its allowed limits, inverter-based generations and droop control of DGs are applied. If they cannot control the frequency in the transition between two operation intervals, load shedding may happen due to tripping of frequency relays. Therefore, the maximum generation variations during the transition between two operation intervals should be calculated and considered in the optimization process. The dynamic model of the system's frequency is given by (4-2), where $\mathrm{H}_{\mathrm{eq}}$ is the microgrid equivalent inertia constant in seconds, $\mathrm{f}$ is the electrical angular velocity or frequency, and $\mathrm{P}_{\mathrm{m}}$ and $\mathrm{P}_{\mathrm{e}}$ are the mechanical and electrical power, respectively. Considering the maximum allowed rate of frequency deviations $(d f / d t)$ in the microgrid and the expected electrical power variations for each interval, the maximum and minimum generation variations over the transition time are calculated by (4-3).

$$
\begin{gathered}
\Delta P_{m}-\Delta P_{e}=2 H_{e q} \frac{d f}{d t} \\
\Delta P_{g}^{\max / \min }=2 H_{e q} \frac{d f^{\min / \max }}{d t}+\Delta P_{e}^{\max / \min }
\end{gathered}
$$

These values determine the generators' variation limits in the islanding mode. However, in grid-connected mode, the frequency and voltage are supported by the utility. In that case, the only constraints for the generators' variations are the operational limits. 


\subsubsection{Game-theoretic double auction mechanism}

A double auction mechanism can be considered as a game. In a GTDAM, buyers and sellers are players. They use their bidding strategies, and the auctioneer determines the MCP. The objective is to find the Nash equilibrium for the game, the situation in which no player has any further changes to apply to its bid/ask price because they can't get more benefit by changing their offers. It is worth mentioning that in this study, buyers try to provide their base demand in this market level to avoid additional payments in the following market levels.

It is assumed that all agents forecast and categorize their demand/generation capability for the next day demand intervals considering all constraints and operational costs (these agents are called followers). These values, along with their price bids, are submitted to the LA, GA, and DA (which are called leaders). Those agents (which are now followers of LMA) sort the data and submit them to the LMA (second leader). The LMA runs the auction to find the MCP and determines the winners and losers in this round of the game. Afterward, the auction outputs are submitted to the followers (LA, GA, and DA), and they inform their followers (loads, DGs and DESSs) to make a decision for the next round of the game. This game could be considered as a static game with incomplete information because it is designed in such a way that players choose their strategies simultaneously

without knowing the exact preferences of the other players. Each market player has its bidding strategy to maximize/minimize its benefits/costs regarding the outputs of the auction. In this subsection, the strategy of different players is presented, and afterward, the Nash equilibrium of GTDAM is described. 


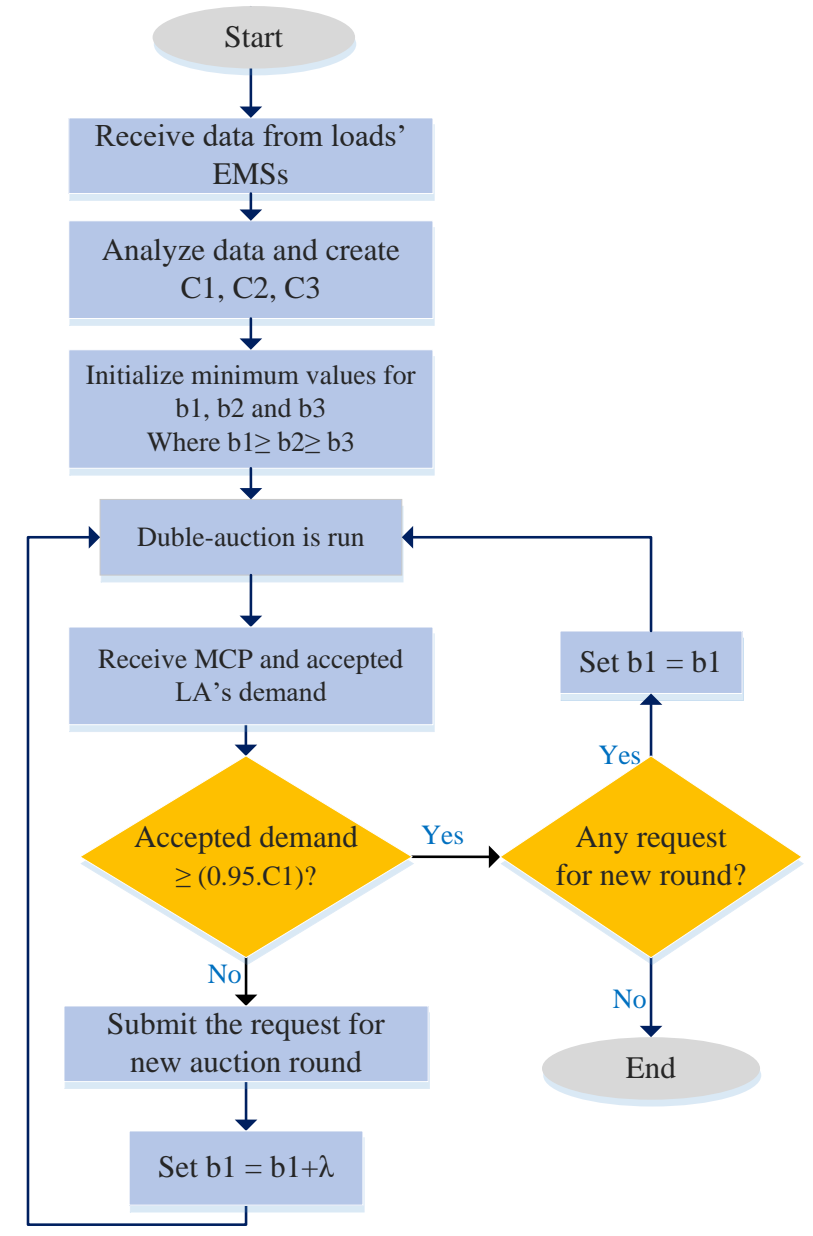

Figure 4.6: LA's bidding strategy for the DAM.

\section{Load Agent Strategy}

Each microgrid may include one or more LA. As described in Section 4.1, LAs are considered as retailers in this structure, which means that they are in charge of some aggregated loads. In other words, they can communicate with EMSs of individual loads to gather data and participate in the electricity market. For this purpose, the LA asks loads' EMSs to submit their forecasted demand in three categories, considering their priorities, which are called Category $1(\mathrm{C} 1)$ of highest priority, Category 2 (C2) of less priority, and Category 3 (C3) of minimum priority. After collecting this data, the LA manages it using the available load shifting considering the energy price and operation time. 

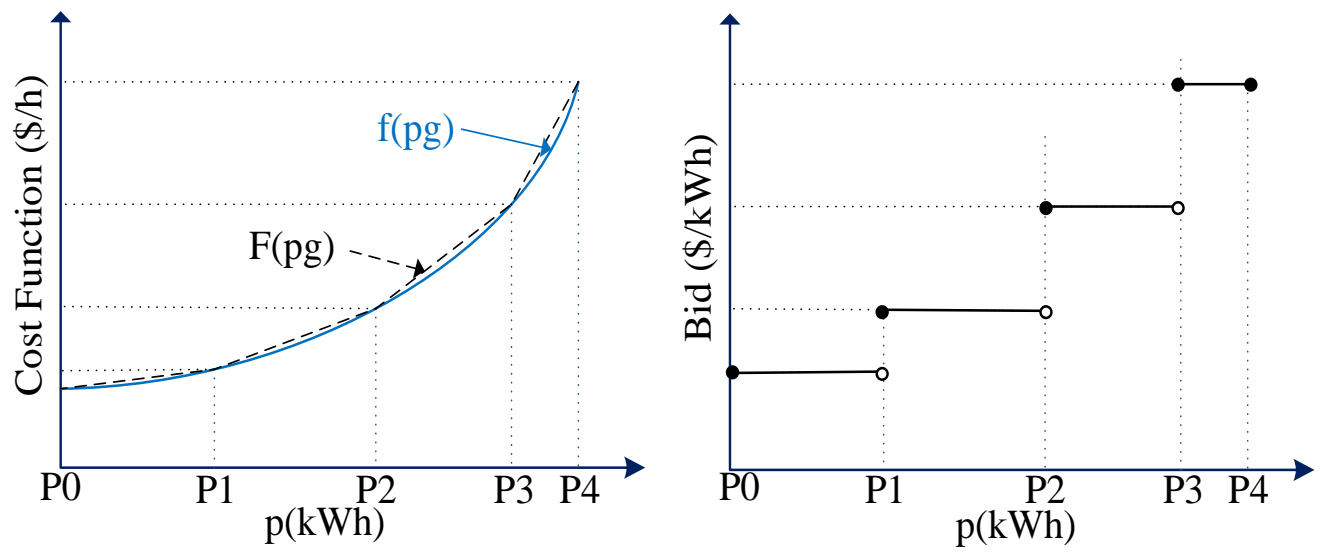

Figure 4.7: Generator's cost function and price bids calculations for participation in the double auction.

Finally, it determines three final aggregated load categories: C1, C2, and C3. The main task of the LA is to provide the base load demand (C1) from the DAM by deploying an effective bidding strategy. In this paper, a simple bidding strategy is used. This is demonstrated in Figure 4.6. In this figure, b1, b2, and b3 are bid offers for C1, C2, and $\mathrm{C} 3$, respectively. $\lambda$ is the bid increment, which is a function of the agent policy, the values of the accepted demand, the previous bid value, and the MCP. In this paper, equation (44) is used to calculate $\lambda$. Where $\varepsilon$ is a positive value determined by the agent strategy.

$$
\lambda=\left\{\begin{array}{cl}
\left(M C P-b_{1}\right) & \text { if } b 1<M C P \\
\varepsilon & \text { if } b 1=M C P
\end{array}\right.
$$

\section{$>$ Generator Agent Strategy}

Each generation unit agent has its specific cost function for each market interval, usually being a non-linear polynomial function of the active power. This function (in $\$ / \mathrm{h}$ ) is presented by (4-5).

$$
f_{t}^{i}\left(p_{g}\right)=a_{t}^{i} \cdot p_{g}{ }^{2}+b_{t}^{i} \cdot p_{g}+c_{t}^{i} \quad i=1,2,3, \ldots, N_{D G}
$$

$f_{t}^{i}\left(p_{g}\right)$ is the energy cost of the $\mathrm{i}^{\text {th }}$ unit at $\mathrm{t}^{\text {th }}$ market interval in $\$ / \mathrm{h}$. Other variables such $a_{t}^{i}, b_{t}^{i}$ and $c_{t}^{i}$ are constant coefficients for this unit in this interval. To participate in the 
DAM and RTM auctions, generation units typically need to send their offers using constant energy bids within a specific generation range [164]. It is possible to convert the non-linear cost function to a linear one as below.

$$
F_{t}^{i}\left(p_{g}\right)=\left(\frac{f_{t}^{i}\left(p_{j}\right)-f_{t}^{i}\left(p_{k}\right)}{p_{j}-p_{k}}\right) \cdot\left(p_{g}-p_{i}\right)+f_{t}^{i}\left(p_{i}\right) \quad, p_{k} \leq p_{g}<p_{j}
$$

The price bids are the first derivative of (4-6); this results in a step function, including several segments as presented in (4-7). Figure 4.7 shows the non-linear cost function, the linear cost function, and the price bids.

$$
\operatorname{Bid}_{t}^{i}(\$ / k W h)=\nabla F_{t}^{i}\left(p_{g}\right) \quad, p_{k} \leq p_{g}<p_{j}
$$

It is worth mentioning that these bid prices are the base and minimum offers. Since the market has a game-theoretic and competitive framework, the generation agents can change their offers using their preferred bidding strategy to maximize their benefits in all market levels. Since DGs can usually change their set-points quickly to follow the load curve, they may reserve some of their capacity for the HAM and RTM levels, forsaking immediate advantages for delayed more significant benefits. Lastly, the GA collects and sorts the data of all DGs in the microgrid in ascending order and submits them to the LMA.

\section{$>$ DESS Strategy}

DESSs are auxiliary resources of microgrids and offer a significant advantage to the DR program because of their flexibility to charge/discharge and their capability to be charged from an external energy resource like solar panels or a wind turbine. A typical EMS of a DESS minimizes the charging cost while maximizing the income from discharging, considering the life degradation cost of the battery and its initial and final SOC. In this paper, DESSs can offer two categories for their available capacities. If the 
DESS is in charging mode $(\Phi=1)$, it will deploy a similar bidding strategy as reported for LA, aiming at providing $100 \%$ of $\mathrm{C} 1$ from the DAM. In discharging mode $(\Phi=-1)$, it can participate in the market like a generation unit, considering its relevant costs. In other words, the submitted bid must cover the degradation cost during the discharging time [184].

\section{Nash equilibrium of GTDAM}

As described in the previous section, LAs and DAs want to provide their base load (C1) from the DAM. Since the utility is considered as an infinite bus, there is at least one Nash equilibrium for this game. It means that if the GAs ask prices are more than the utility price, buyers can buy their base demand by the utility price. Otherwise, the Nash equilibrium is a state in which the accepted bid of each LA is more than $95 \%$ of its C1, and for each DA, it should meet $100 \%$ of $\mathrm{C} 1$. Afterward, the auction outputs are reported through the MAS to the market players to prepare their bids for the HAM.

\subsubsection{Level 2 - Hour-Ahead Market}

The HAM is the second level of this market framework, where loads, DGs, and DESSs have a better estimation of their demand and generation for the next hour of operation. The LMA asks these agents to submit their forecasted mismatches, DR capabilities, cost functions, and their availability for the upcoming period of operation. Each agent, at this point, uses its strategy to minimize its costs or maximize its revenues, considering its constraints. In fact, instead of solving a big optimization problem in the LMA considering all agents' constraints, load shifting, and DESSs' flexibilities, the LMA (leader) asks the agents (followers) to submit their participation and bids. This method decreases the optimization complexity and solution time while encouraging all market players to participate in the DR program to minimize the energy mismatches in the system. The 
developed optimization model reduces the forecasted mismatch cost at $t^{\text {th }}$ HAM interval, as shown in (4-8).

$$
\operatorname{Min}\left(\begin{array}{l}
\left|\Delta p_{t}^{r}\right| \cdot C_{u, t}^{f}+\sum_{l=1}^{N_{L A}} \alpha_{l, t} \cdot C\left(\Delta p_{l, t}\right)+ \\
\sum_{d=1}^{N_{D E S S}} \alpha_{d, t} \cdot C\left(\Delta p_{d, t}\right)+\sum_{g=1}^{N_{g}} \alpha_{g, t} \cdot C\left(\Delta p_{g, t}\right)
\end{array}\right)
$$

Subject to:

$$
\begin{array}{cc}
\Delta p_{l, t}^{-} \leq \Delta p_{l, t} \leq \Delta p_{l, t}^{+} & l=1,2,3, \ldots, N_{L A} \\
\Delta p_{d, t}^{-} \leq \Delta p_{d, t} \leq \Delta p_{d, t}^{+} & d=1,2,3, \ldots N_{d} \\
\Delta p_{g, t}^{-} \leq \Delta p_{g, t} \leq \Delta p_{g, t}^{+} & g=1,2,3, \ldots, N_{g}
\end{array}
$$

Where, $\Delta p_{t}^{r}$ is the remaining mismatch in $\mathrm{kWh}$ after clearing the HAM, $\alpha_{l, t}, \alpha_{d, t}$ and $\alpha_{g, t}$ are binary inputs showing the availability of Loads, DESSs and DGs for participation in the HAM, respectively. $C\left(\Delta p_{l, t}\right), C\left(\Delta p_{d, t}\right)$, and $C\left(\Delta p_{g, t}\right)$ are the cost functions in $\varnothing$ for rescheduling reported by LA, DA and GA to participate in the HAM, respectively and $C_{u, t}^{f}$ is the utility price in $\varnothing / \mathrm{kWh}$ which is forecasted by the LMA. The (-) and (+) in (4-9), (410) and (4-11) show the minimum and maximum limits for the variables, respectively. $\Delta p_{l, t}, \Delta p_{d, t}$ and $\Delta p_{g, t}$ are in $\mathrm{kWh}$ and are defined as changes in the active power of $1^{\text {th }} \mathrm{LA}$, $\mathrm{d}^{\text {th }}$ DESS and $\mathrm{g}^{\text {th }}$ DG, respectively. $N_{L A}, N_{d}$ and $N_{g}$ are the number of LAs, DESSs and DGs in the microgrid, respectively. In (8), $\Delta p_{t}^{r}$ is calculated by (4-12).

$$
\Delta p_{t}^{r}=\Delta p_{t}^{f H}+\sum_{l=1}^{N_{L A}} \alpha_{L, t} . \Delta p_{l, t}+\sum_{d=1}^{N_{d}} \alpha_{d, t} . \Delta p_{d, t}+\sum_{g=1}^{N_{g}} \alpha_{g, t} \cdot \Delta p_{g, t}
$$

Where, $\Delta p_{t}^{f H}$ is the total forecasted mismatch in $\mathrm{kWh}$ for $\mathrm{t}^{\text {th }}$ HAM interval and is calculated by (4-13). 


$$
\Delta p_{t}^{f H}=\sum_{l=1}^{N_{L A}} \Delta p_{l, t}^{f}+\sum_{d=1}^{N_{d}} \phi_{d, t} . \Delta p_{d, t}^{f}-\sum_{g=1}^{N_{g}} \Delta p_{g, t}^{f}
$$

In (4-13), $\Delta p_{l, t}^{f}, \Delta p_{d, t}^{f}$ and $\Delta p_{g, t}^{f}$ are the forecasted mismatches in $\mathrm{kWh}$ reported by $\mathrm{l}^{\text {th }}$ LA, $\mathrm{d}^{\text {th }}$ DESS and $\mathrm{g}^{\text {th }}$ DG, respectively. $\phi_{d, t}$ is a binary input which shows the operation mode of the DESSs, which is (1) if the DESS is in charging mode and (-1) if it is in discharging mode. In this paper, cost functions for rescheduling are defined by (4-14).

$$
C\left(\Delta p_{x, t}\right)=C_{x, t} \cdot\left|\Delta p_{x, t}\right|, \quad C_{x, t}=\left\{\begin{array}{lll}
C_{x, t}^{+} & \text {if } & \Delta p_{x, t}>0 \\
C_{x, t}^{-} & \text {if } & \Delta p_{x, t} \leq 0
\end{array}, \quad x \in\right. \text { Agents }
$$

Where $C_{x, t}^{+}$and $C_{x, t}^{-}$are in $\phi / \mathrm{kWh}$ and are defined as energy prices for positive and negative changes, respectively. This optimization minimizes the cost of imbalance in the microgrid by comparing the components' bids with the forecasted utility price. The cost of this market clearing will be imposed on the components, which have reported their power mismatches regarding their contributions.

\subsubsection{Level 3 - Real-Time Market}

The last level of the developed market is the RTM. In this paper, real-time is defined as five minutes-ahead market. As the algorithm approaches the operation time, all agents may have a better estimation of their load demands/generation capacities and flexibilities (DR, generation variations) during the upcoming interval. Using updated data for each agent, running a fast inter-microgrid market aiming at minimizing the net area energy mismatch becomes possible. This interaction between microgrids is done by deploying a reverse-auction mechanism in the area comprising several microgrids connected to the PCC. The reverse auction mechanism was described in detail in [43]. In this mechanism, sellers compete to sell their goods to a buyer. 
In this dissertation, the load DR is also considered in the RTM. For this purpose, it is considered as a generation unit when DR program decides to decrease the load demand. In this market level, the GMA (leader) asks LMAs (followers) to send their forecasted mismatches, errors, and their components' participations and price bids for the RTM. Subsequently, the GMA calculates the net area mismatch as in (4-15).

$$
\Delta p_{t}^{N e t}=\sum_{i=1}^{N_{m}} \Delta p_{t}^{i}
$$

Where, $N_{m}$ is the number of microgrids in the area, $\Delta p_{t}^{N e t}$ and $\Delta p_{t}^{i}$ are in $\mathrm{kWh}$, representing the net area energy mismatch and total mismatch reported by $\mathrm{i}^{\text {th }}$ microgrid, respectively. $\Delta p_{t}^{i}$ is defined by (4-16).

$$
\Delta p_{t}^{i}=\Delta p_{t}^{f R}+\Delta p_{t}^{r}+\Delta p_{t}^{E} i=1,2,3, \ldots, N_{m}
$$

Where $\Delta p_{t}^{f R}$ is the total forecasted mismatch in $\mathrm{kWh}$ for $\mathrm{t}^{\text {th }} \mathrm{RTM}$ interval and is calculated just like $\Delta p_{t}^{f H}$ in (4-13) considering the updated values by agents for the RTM. $\Delta p_{t}^{r}$ and $\Delta p_{t}^{E}$ are in $\mathrm{kWh}$ and are defined as the remaining mismatch from the HAM market and the estimated error forecasted by LMA for this operation interval, respectively. In this reverse auction model, DGs, DESSs and LAs from all microgrids in the area compete together to meet the net power mismatch by minimum price.

Figure 4.8 shows the mechanism of this market. The GMA sorts the data of the sellers in increasing order of their bids. The intersection of the sellers' curve and the energy mismatch value $\left(U_{m}\right)$ is the output of this auction, as shown by (4-17).

$$
\min \int_{0}^{U_{m}} S(u) d u
$$




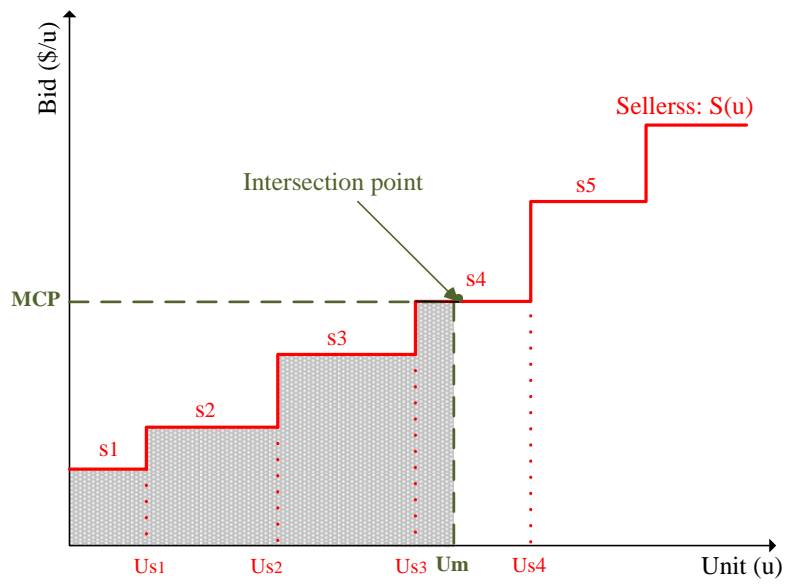

Figure 4.8: Reverse auction mechanism indicating the competition between energy providers in the RTM.

The MCP, which is determined by the intersection point of the mismatch value and the sellers' curve $\left(U_{m}, M C P\right)$, is the output of this optimization. If the reported capacities for this market level are not enough for compensating the net area mismatch or if the bid offers are more than the utility price, the surplus imbalance will be assigned to the utility. It means that this game has at least one Nash equilibrium. In islanding mode, when the PCC is disconnected from the power grid, the total imbalances will be compensated using the droop control strategy or by load shedding. Outputs of this optimization are submitted to the LMAs and they send the final set-points to all agents in that microgrid as described in Figure 4.4. The market is cleared by charging the players that caused mismatches and paying the players that contributed in the RTM according to their contribution.

\subsection{Numerical results}

In this dissertation, we selected and modified the IEEE 37-bus distributed network as a test system to represent a multi-microgrid area containing three microgrids [14]. In this study, Four DESSs are added to this system, as shown in Figure. 4.9, and DIgSILENT PowerFactory 2017 was used to develop the computational part of the agents using DPL. 


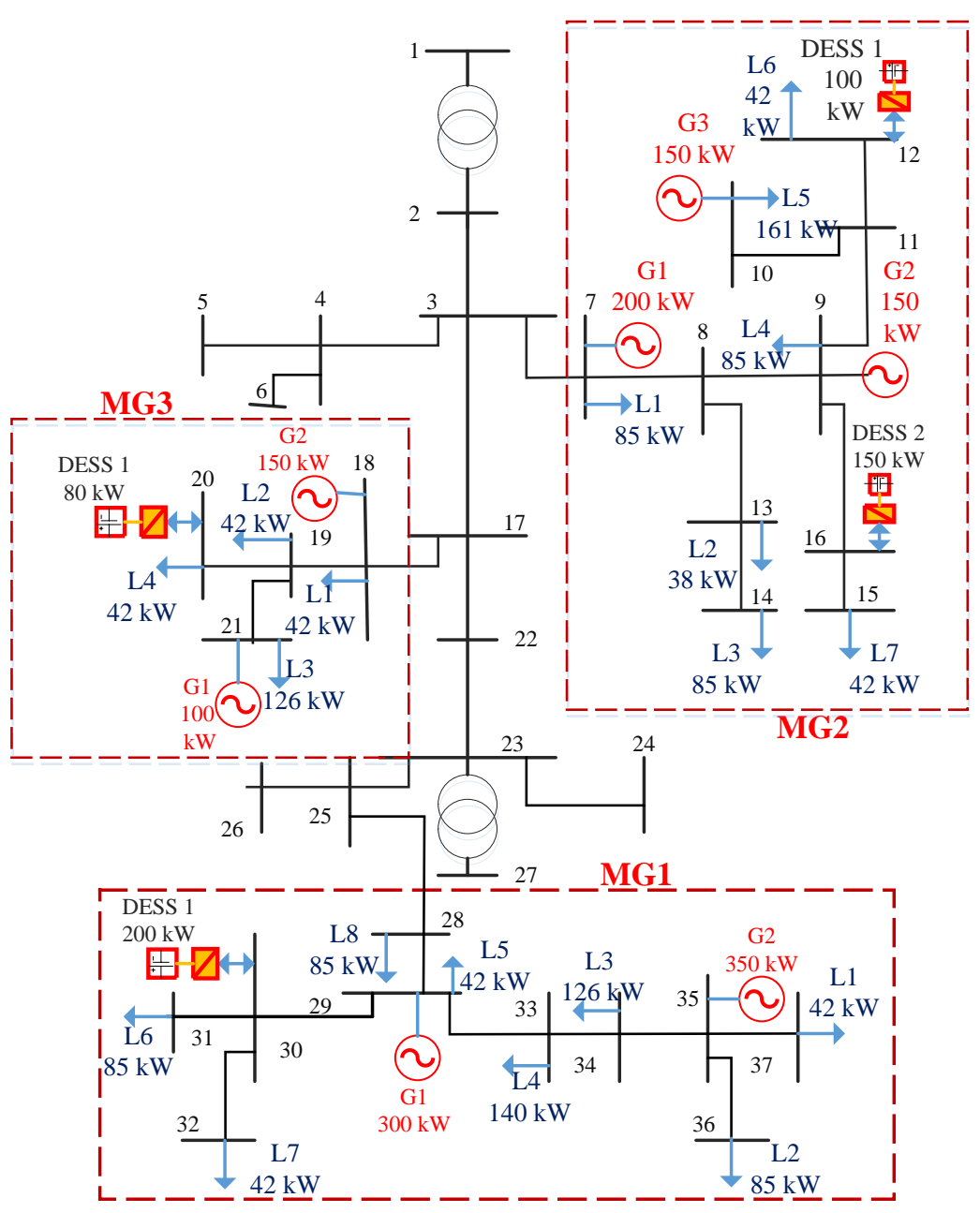

Figure 4.9: The modified IEEE 37-bus distributed network including three MGs in grid-connected mode

Communication

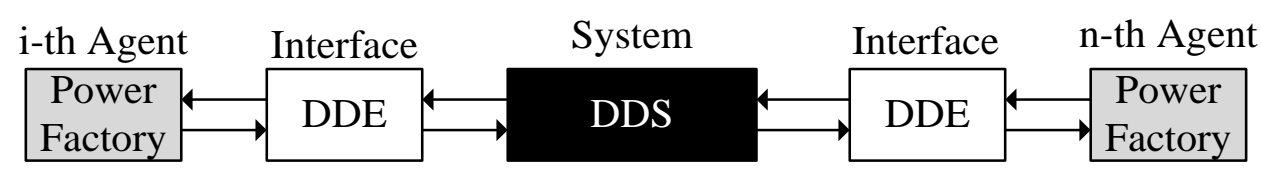

Figure 4.10: Interface between agents developed in DigSilent PowerFactory and DDS through DDE.

The reason is that by using DigSilent, it is possible to analyze and simulate the system conditions and constraints for each market interval. Therefore, on the hosting computers of all agents (LAs, DAs, GAs, LMAs, Utility, and GMA), the software is installed, and the DDE is activated as an interface between agents and the DDS, as shown in Fig. 4.10. Numerical results are presented in this section for the three market levels in detail. 


\subsubsection{Day ahead market}

In the DAM, a game-theoretic double auction mechanism is run. Thus, the offers from LAs and DESSs (with $\Phi=1$ ) are sorted in descending order while suppliers' offers are sorted in incremental order, and the intersection point of these two curves determines the MCP. This process is repeated in all three LMAs in parallel to find the MCP and returning to all market players regarding the winners, losers, and the accepted demands and supplies. By receiving the auction results, market agents may request a new auction round by offering new price bids using their bidding strategy algorithm.

In this dissertation, LAs and DESSs (with $\Phi=1$ ) actively participate in this game utilizing their bidding strategy algorithm (which was described in Section 4.3.1.2). For the sake of simplicity, we assume that suppliers (DGs and DESSs with $\Phi=-1$ ) do not change their bids for new rounds of the game in each DAM interval. The results of this market for $9^{\text {th }}$ and $13^{\text {th }}$ market intervals in MG1 are shown in Figure 4.11. The step by step numerical results for these intervals and the market clearing process to find the Nash equilibrium of the game is reported in Table 4.1.

Table 4.1: DAM results for $9^{\text {th }}$ and $13^{\text {th }}$ intervals.

\begin{tabular}{|c|c|c|c|c|c|c|}
\hline$t=9$ & \multicolumn{2}{|c|}{ Bid (\$/kWh) } & \multicolumn{2}{|c|}{ Accepted Demand (\%) } & \multicolumn{2}{|c|}{ Auction Results } \\
\hline Round & LA & DESS & LA-C1 & DA-C1 & MCP & New round? \\
\hline 1 & 9.2 & 9.5 & 71.05 & 100.00 & 9.2 & Yes \\
\hline 2 & 9.5 & 9.5 & 97.37 & 100.00 & 9.5 & No \\
\hline$t=13$ & \multicolumn{2}{|c|}{ Bid (\$/kWh) } & \multicolumn{2}{|c|}{ Accepted Demand (\%) } & \multicolumn{2}{|c|}{ Auction Results } \\
\hline Round & LA & DESS & LA-C1 & DA-C1 & MCP & New round? \\
\hline 1 & 9.5 & 11 & 64.81 & 100.00 & 9.5 & Yes \\
\hline 2 & 11 & 11 & 100.00 & 0.00 & 11 & Yes \\
\hline 3 & 11 & 13 & 88.89 & 100.00 & 11 & Yes \\
\hline 4 & 13 & 13 & 100.00 & 87.50 & 13 & Yes \\
\hline 5 & 13 & 13.5 & 98.15 & 100.00 & 13 & $\mathrm{No}$ \\
\hline
\end{tabular}


DAM, MG1, $\mathrm{t}=9$

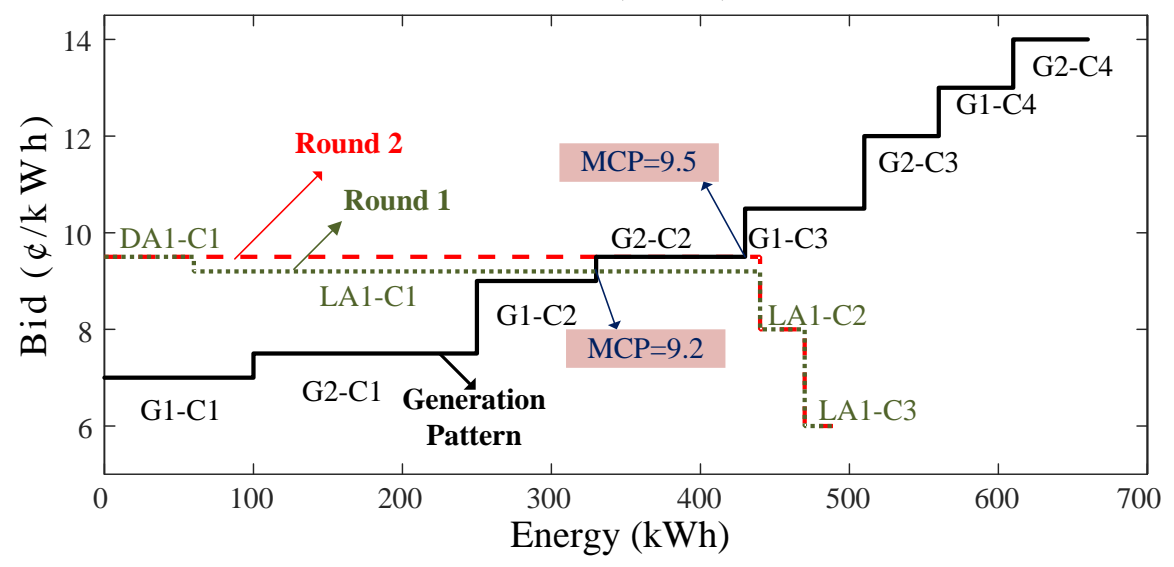

DAM, MG1, t=13

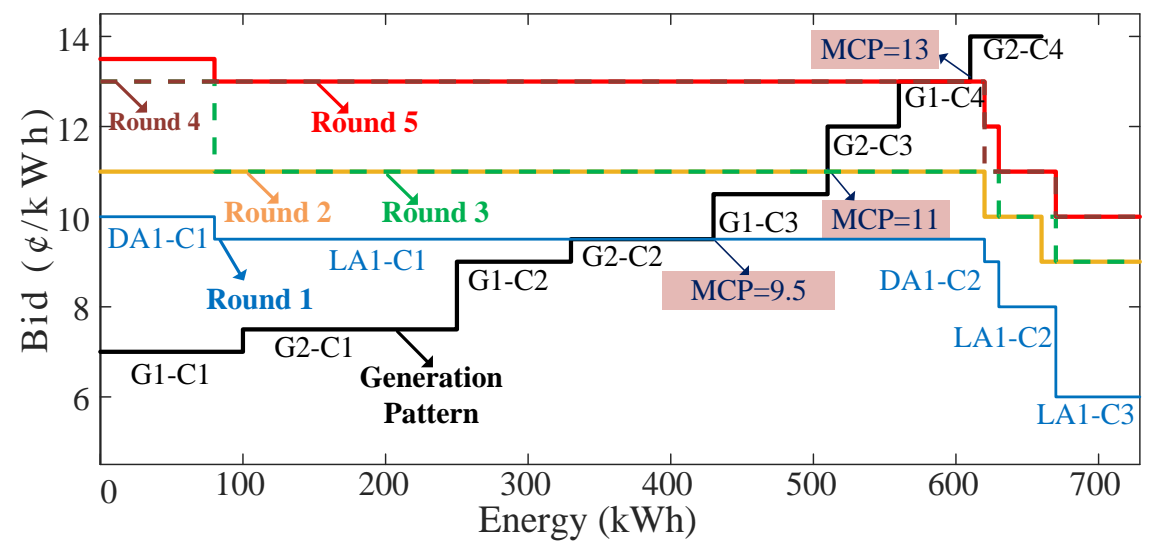

Figure 4.11: The Game theory based DAM results for MG1 at $9^{\text {th }}$ and $13^{\text {th }}$ market intervals.

The results show that the game stops after two and five rounds for $\mathrm{t}=9$ and $\mathrm{t}=13$, respectively. In these cases, it can be observed that both demand-side players (LA and DESS) stop requesting a new auction round when they meet their criteria. As mentioned before, there criteria for a LA is to provide more than $95 \%$ of its base load and for a DESS is to provide $100 \%$ of its base load from the DAM. In Table 4.2, the DAM results for all three MGs are reported for the 9th market interval. Figure 4.12 shows the load profiles and DESSs' demand/generation values in a 24-hour market interval for all three MGs. 
Table 4.2: The data flow and market results for $9^{\text {th }}$ interval.

\begin{tabular}{|c|c|c|c|c|c|c|c|c|c|c|c|c|c|c|}
\hline Microgrid & \multicolumn{4}{|c|}{ MG1 } & \multicolumn{6}{|c|}{ MG2 } & \multicolumn{4}{|c|}{ MG3 } \\
\hline Agent & LA1 & DG1 & DG2 & $\begin{array}{c}\text { DESS1 } \\
\Phi=1\end{array}$ & LA1 & DG1 & DG2 & DG3 & $\begin{array}{c}\text { DESS1 } \\
\Phi=1\end{array}$ & $\begin{array}{c}\text { DESS2 } \\
\Phi=-1\end{array}$ & LA1 & DG1 & DG2 & $\begin{array}{c}\text { DESS1 } \\
\Phi=1\end{array}$ \\
\hline Market & \multicolumn{14}{|c|}{ DAM } \\
\hline $\begin{array}{l}\text { Requested } \\
(\mathrm{kWh})\end{array}$ & 439 & 300 & 350 & 60 & 413 & 200 & 200 & 150 & 80 & 100 & 203 & 100 & 150 & 59 \\
\hline $\begin{array}{l}\text { Accepted } \\
(\mathrm{kWh})\end{array}$ & 370 & 180 & 250 & 60 & 339 & 150 & 100 & 50 & 61 & 100 & 180 & 100 & 125 & 45 \\
\hline $\operatorname{MCP}(\phi / k W h)$ & \multicolumn{4}{|c|}{9.5} & \multicolumn{6}{|c|}{9.75} & \multicolumn{4}{|c|}{11} \\
\hline Cost $(\phi)$ & 3515 & -1710 & -2375 & 570 & 3305 & -1462 & -975 & -487 & 594 & -975 & 1980 & -1100 & -1375 & 495 \\
\hline Market & \multicolumn{14}{|c|}{ HAM } \\
\hline$\Delta P_{t}^{f H}(\mathrm{~kW})$ & 20 & 0 & 0 & 0 & 14 & 0 & 0 & 0 & 0 & -20 & 0 & 0 & 0 & 15 \\
\hline$\Delta P_{t}^{-}(\mathrm{kW})$ & $*$ & -50 & -50 & -20 & $*$ & -50 & -50 & 0 & -10 & $*$ & -10 & -50 & -75 & $*$ \\
\hline$\Delta P_{t}^{+}(\mathrm{kW})$ & $*$ & 60 & 50 & 120 & $*$ & 25 & 20 & 50 & 39 & $*$ & 20 & 0 & 25 & $*$ \\
\hline$C_{t}^{-}(\phi / \mathrm{kWh})$ & $*$ & 0 & 0 & 14 & $*$ & 0 & 0 & 0 & 14 & $*$ & 14 & 0 & 0 & $*$ \\
\hline$C_{t}^{+}(\phi / \mathrm{kWh})$ & $*$ & 10.5 & 12 & 0 & $*$ & 11 & 13 & 12 & 0 & $*$ & 0 & 0 & 13 & $*$ \\
\hline$\alpha_{t}$ & 0 & 1 & 1 & 1 & 0 & 1 & 1 & 1 & 1 & 0 & 1 & 1 & 1 & 0 \\
\hline$\Delta P_{t}(\mathrm{~kW})$ & 0 & 20 & 0 & 0 & 0 & 25 & 0 & 9 & 0 & 0 & 0 & 0 & 15 & 0 \\
\hline Set-Point $(\mathrm{kW})$ & 390 & 200 & 250 & 60 & 353 & 175 & 100 & 59 & 61 & 80 & 180 & 100 & 140 & 60 \\
\hline MCP $(\notin / k W h)$ & \multicolumn{4}{|c|}{10.5} & \multicolumn{6}{|c|}{11.26} & \multicolumn{4}{|c|}{13} \\
\hline $\operatorname{Cost}(\phi)$ & 105 & -105 & 0 & 0 & 157 & -281 & 0 & -101 & 0 & 225 & 0 & 0 & -195 & 195 \\
\hline Market & \multicolumn{14}{|c|}{ RTM } \\
\hline$\Delta P_{t}^{f R}(\mathrm{~kW})$ & 15 & 0 & 0 & 0 & 15 & 0 & 0 & 0 & 12 & 0 & 0 & 0 & 0 & 0 \\
\hline$\Delta P_{t}^{E}(\mathrm{~kW})$ & \multicolumn{4}{|c|}{3} & \multicolumn{6}{|c|}{5} & \multicolumn{4}{|c|}{4} \\
\hline$\Delta P_{t}^{ \pm}(\mathrm{kW})$ & $*$ & 20 & 20 & -10 & $*$ & 5 & 15 & 20 & $*$ & 0 & -10 & 0 & 10 & 0 \\
\hline Bid $(\phi / \mathrm{kWh})$ & $*$ & 12 & 13 & 14 & $*$ & 16 & 15 & 14 & $*$ & 0 & 16 & 0 & 15 & 0 \\
\hline$\Delta P_{t}(\mathrm{~kW})$ & 0 & 20 & 20 & -10 & 0 & 0 & 0 & 4 & 0 & 0 & 0 & 0 & 0 & 0 \\
\hline MCP $(\varnothing / k W h)$ & \multicolumn{14}{|c|}{14} \\
\hline Cost $(\varnothing)$ & 210 & -280 & -280 & -140 & 210 & 0 & 0 & -56 & 168 & 0 & 0 & 0 & 0 & 0 \\
\hline Market & \multicolumn{14}{|c|}{ Market Clearing } \\
\hline Set-Point (kW) & 405 & 220 & 270 & 50 & 368 & 175 & 100 & 63 & 73 & 80 & 180 & 100 & 140 & 60 \\
\hline Cost $(\varnothing)$ & 3830 & -2095 & -2655 & 430 & 3672 & -1743 & -975 & -664 & 672 & -750 & 1980 & -1100 & 1570 & 690 \\
\hline
\end{tabular}

The DAM results, including the base load value $(\mathrm{C} 1)$ for each MG and the MCPs, are presented in Figure 4.13. It can be observed that three different load profiles in three MGs result in three different MCPs in the DAM.

\subsubsection{Hour ahead market}

The LMA of each MG runs its HAM to improve the scheduling and minimize the forecasted mismatch within its microgrid. The effectiveness of the developed hierarchical optimization process, along with a detailed data flow for the $9^{\text {th }}$ market interval, is reported for all MGs in Table 4.2. From the results, it is observed that those market players who have reported mismatches are charged by the LMA. 

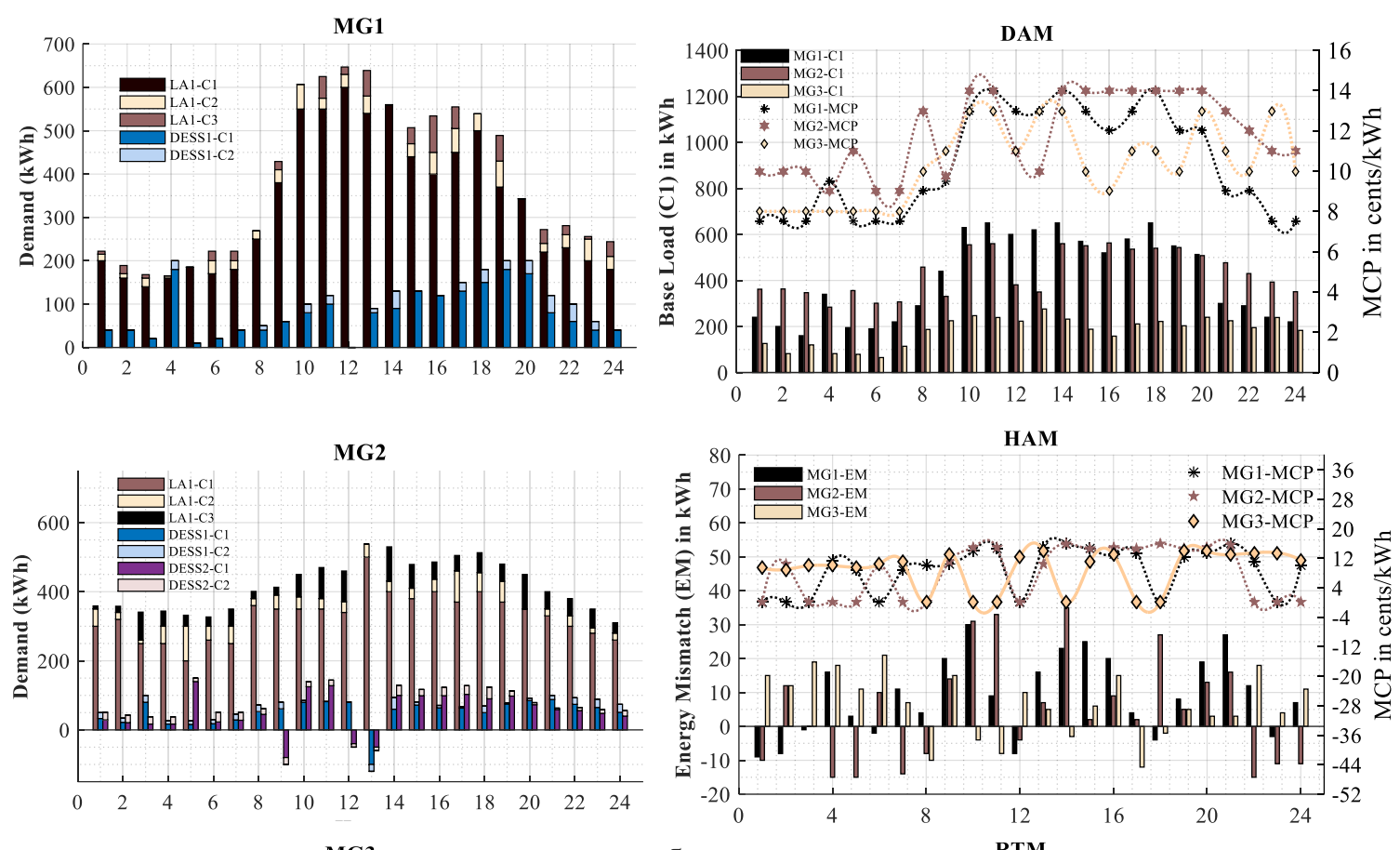

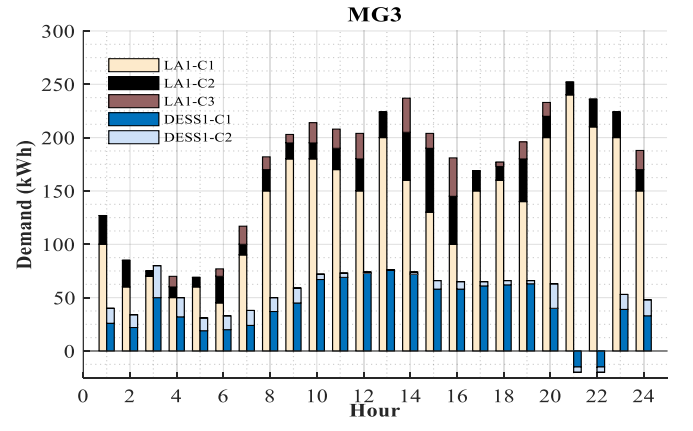

Figure 4.12: load and generation profiles

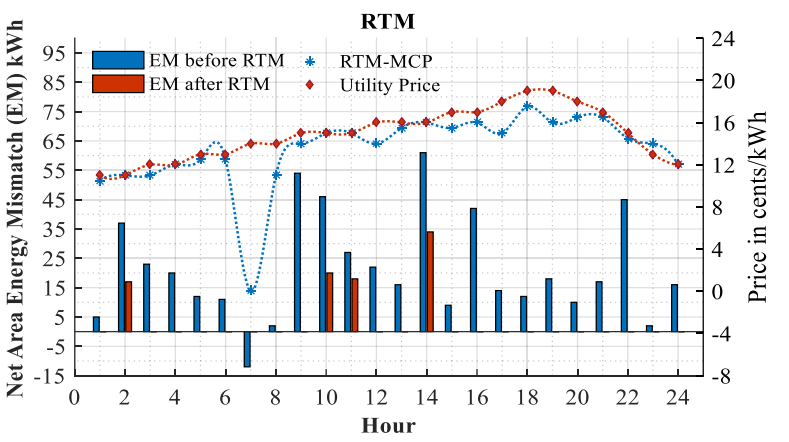

Figure 4.13: DAM, HAM and RTM result

The charging price in MG1 is $10.5 \notin / \mathrm{kWh}$ (equal to HAM clearing price), which is around $10 \%$ more than the MCP in DAM $(9.5 \phi / \mathrm{kWh})$. It can be concluded that better load forecasting can decrease the energy costs for market players. It is not only because of purchasing the energy by the lower price but also due to the participation in the DR program. The market players who have not reported mismatches can participate in the DR, and therefore, they will get paid by the LMA depending on their contributions. Figure 4.13 illustrates the forecasted mismatch in each MG and the relevant MCP in the HAM during a day. It is worth mentioning that this market level would not experience the market power 
for its players because the utility price is the limit for the price bids, and it can adjust the dependency on the network. For example, during peak load hours, when the utility price is maximum, the developed algorithm automatically minimizes the participation of the utility by purchasing the power from the microgrid's suppliers. During off-peak load hours, when big generation companies in the power system can provide electric power at a lower price, a competition starts between the microgrid resources and the utility. Moreover, the islanding operation mode of the microgrids can be modeled in this market by choosing a high price for the utility, which will result in the minimum assigned value to the utility network. In other words, load shedding is minimized in islanding mode.

It should be noted that three LMAs are running their optimization in parallel. Therefore, each LMA solves a partial optimization problem that could be solved very fast (less than 30 seconds). However, if the optimization method introduced in [50] is deployed, the HAM solution time will be around 31, 36, and 22 minutes for MG1, MG2, and MG3, respectively. Although the mentioned approach in this reference considers all system constraints and components cost/benefits in one optimization problem, it does not allow the agents to operate independently in the market. Furthermore, if the scale of the system increases, the solution time would rise significantly. Because of the long solution time obtained by the proposed method in [165], it is not possible to consider the participation of DESSs in the real-time market to find a solution for the optimization problem.

In this dissertation, the fast solution time obtained by the developed hierarchical solution method allows us to implement the participation of DESSs and DGs in the realtime inter-microgrid market before the operation time, aiming at minimizing the real-time mismatch in the area, optimally. 


\subsubsection{Real-time market}

The RTM is aimed to minimize the net area mismatch by running an inter-microgrid reverse auction model. In this study, the RTM is run 5 minutes before the operation time. After receiving all mismatches and bid offers from market players for the RTM, the GMA calculates the net area mismatch and, based on this value, runs the reverse auction model to minimize the mismatch with minimum cost. It should be noted that if we do not run the RTM, any reported mismatch for market players after the HAM market will be assigned to the utility.

In this market, like HAM and DAM, all market players can participate. Figure 4.14 indicates the reverse auction results for the $9^{\text {th }}$ RTM interval. The numerical results are correspondingly reported in Table 4.2. The results show that the net area mismatch is 54 $\mathrm{kWh}$ in the RTM. Although $18 \mathrm{kWh}$ of this mismatch is reported by MG1, the total compensation of this MG is $50 \mathrm{kWh}$, which means that this MG is selling $32 \mathrm{kWh}$ energy to MG2 and MG3. This energy demand in MG1 is compensated by DG1 and DG2 generations and DESS1 demand reduction. The remaining $4 \mathrm{kWh}$ are provided by DG3 in MG2.

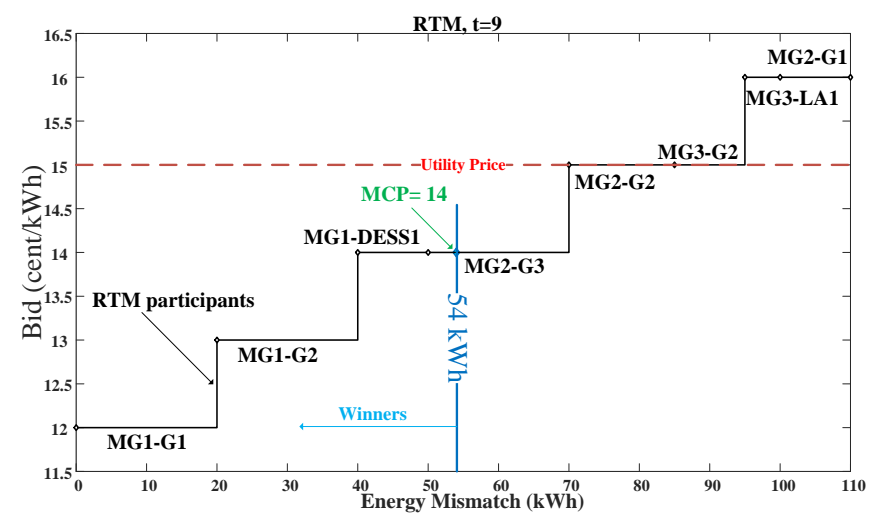

Figure 4.14: Reverse auction result for $9^{\text {th }}$ RTM interval. 
In Figure 4.14, the RTM results for the 24-hour market intervals are reported. From the results, it is concluded that the RTM could successfully remove the dependency on the utility in 20 intervals. The support of the external grid was just needed for four market intervals $(\mathrm{t}=2,10,11$, and 14$)$, where the RTM clearing price is equal to the utility price. Generally, the total reported mismatches for the RTM in this 24-hour interval are 533 and $89 \mathrm{kWh}$ before and after running the RTM, respectively. It means that the dependency on the network is reduced by $83 \%$ when the developed inter-microgrid market is deployed. From the results reported in Table 4.2 and Figure 4.13, it can be seen that at each market interval (t), the MCP increases gradually in the subsequent market levels.

$$
M C P_{i}^{R T M} \geq M C P_{i}^{H A M} \geq M C P_{i}^{D A M} \quad i=1,2,3, \ldots, N_{M G}
$$

Therefore, better load forecasting by agents will decrease the operating costs for them. Finally, it can be observed that the developed market framework is highly successful in developing an optimal open-access energy market to both provide the energy in a competitive environment and minimize the mismatches forecasted by all agents hierarchically through a MAS framework.

\subsection{Summary}

In this Chapter, an optimal multiagent-based market algorithm for smart multimicrogrid systems was developed. In this algorithm, a game-theoretic double auction mechanism was introduced for the DAM, where participants compete to be winner in the auction model and meet their internal demands or their benefits from selling the energy. This market is being repeated until the Nash equilibrium is reached, where none of participants request for new round of the auction. Afterward the HAM market is run to mitigate the energy mismatches within all MGs through an optimization algorithm aiming 
at minimum energy price. The final market is called RTM which is run very close to operating point to minimize the whole area mismatches and consequently the nned for utility support through an inter-MG market by a reverse auction model.

The results demonstrated that using the established market platforms, the autonomous microgrids could optimally use the capabilities of all agents to not only decrease the cost of energy but also to increase the system resiliency in a deregulated environment. The numerical results showed the effectiveness of the developed market framework in successfully achieving the low solution time and minimum dependency on the utility for autonomous multi-microgrid operation. More detail and data are in Appendix I. 


\section{Chapter 5 Secure Communication Platform for Decentralized Electricity Market}

In the shift from the traditional power grid to the smart grid, the design of the communication infrastructure becomes a critical task because a multi-microgrid system requires communication with geographically dispersed devices that are associated with dynamic and decoupled smart grid applications. For example, market auctions, demand response applications, and optimization problems. Therefore, the communication platform should be designed to work over wireless networks and cover various protocols to address the interoperability of different systems. In other words, the middleware must be chosen wisely, as it provides an abstract platform that rides through format-related issues and facilitates both interoperability and scalability [166].

In the widely used communication networks in power system applications, two main distinctions can be identified [167]. The first is based on the networking architecture: client-server, peer-to-peer, and RTPS communication models. The second is between message-centric and data-centric communication approaches. Client-server communication networks that are usually adopted in industry SCADA systems are centrally managed by a server, representing a single point of failure to the system. In those types of networks, communication is usually initiated by the clients, thus invoking servers to provide services. In peer-to-peer communication, on the other hand, each participant has both client and server capabilities, thus eliminating the need for the centralized decision of the server. However, applications that are built based on this communication model are inflexible, and any scalability attempts are strictly limited.

A solution to this is the publish-subscribe communication model, which eliminates the need for the centralized decision of a central broker and helps to achieve a scalable 
distributed environment in the addressed application. Publishers do not send messages to specific receivers or subscribers, as in the peer-to-peer communication model, and subscribers are unaware of the publishers. They subscribe to receive messages.

Therefore, in this Chapter, a secure, reliable, and low latency communication platform is developed for the developed decentralized electricity market presented in Chapter 4. This platform is a data-centric approach that utilizes a RTPS protocol for communication among agents through the DDS as a communication middleware. This approach also facilitates redundancy, a feature needed when building a multiagent system. If one agent fails, the redundant one will readily step into the playground. The DDS also allows the application participants or agents (publishers and subscribers) to start in any order and dynamically connect or disconnect from the network at any time. This feature helps promote the scalability of the system, without any interruption in the microgrid operation. Modifying the developed platform and deploying a proper security measure are other topics which are discussed in this chapter.

\subsection{Message-Centric vs. Data-Centric communication approaches}

The communication networks can be classified into message-centric and data-centric approaches, where the main difference between them is the degree of awareness of the infrastructure to the data space and its involvement in applying modifications to it [168]. In the message-centric approach, the infrastructure ensures it delivers the message, without being aware of its content, and leaves the other jobs such as message formatting and filtering to the end-applications. In the data-centric approach, on the other hand, the middleware is more involved, as it manages the messages and makes them available to the end-applications using standard formats. 


\subsubsection{Message centric communication approach}

The message centric communication approach is used by traditional communication middleware, where a set of messages and data formats are pre-defined in participants' nodes. In this approach, the communication middleware delivers the message through the network layer and does not know the message contents and data types. Messages are being praised within each node of the system (at the application layer) to check the correctness, integrity, data type, and filter data. It means that each node should locally track the state of data, which significantly increases the processing tasks on the application layer. Furthermore, the system expansion is very complicated because significant changes are required on the application layer due to any change in message formats or data types [166].

\subsubsection{Data centric communication approach}

Nowadays, modern communication middleware uses the data-centric approach, in which the middleware builds the message and updates the system state. Therefore, it is aware of the message content and data model [169]. Moreover, instead of processing the messages in the application layer locally, as is done in message centric approach, in a datacentric approach, messages are being processed within the middleware layer to assess the correctness of data types delivered to all nodes. It simplifies application development and system expansion. Additionally, it can enhance the system reliability, optimize the use of network bandwidth and give us more opportunities for assigning different QoS profiles, priorities and security measures to the data types comparing with message centric approach [168]. In this dissertation, the DDS is used as a data-centric communication middleware to address the communication challenges in electricity markets. In the following section, DDS is described, and its unique features are mentioned in detail. 


\subsection{Data Distribution Service (DDS)}

The DDS is a data-centric communication standard that was initially released by the OMG in 2004 [170]. The DDS deploys a DCPS protocol within a virtual global data space to facilitate communication among participants [171]. The DCPS protocol is based on defining topics with specific data types and formats through IDL files over a DDS domain. Afterward, participants with data writer could publish into these topics, and subscribers could use a data reader to receive data. It is worth mentioning that several subscribers would be able to collect data simultaneously once data is written or updated on a topic. Therefore, it is a multicast communication framework that facilitates the data exchange among participants with the same domain ID. This communication platform is fast, expandable, and reliable enough and could be utilized in critical real-time applications. The automatic discovery feature of the DDS makes it possible for dynamic participants to be added/deleted to/from the system without any interruption in the system operation [172]. The DDS has also been equipped with an extraordinary governance and management tool, called QoS, for flexible communication and controlling the system behavior in terms of data latency budget, priority order, lifespan, durability, etc. The single point of failure feature in most of the message centric communication middleware directly affects the system reliability. However, the DDS does not need a message broker for peer-to-peer communication, and therefore, it is an excellent and highly reliable tool for distributed applications.

In addition to the high reliability of the DDS, it is easily scalable through the DDS routing service for distributed applications with participants in different networks and diverse transport protocols (e.g., IPv4, IPv6, or shared memory) [173]. This service is 
achievable through the QoS profile, where the IP address of participants, along with proper network protocol (e.g., TCP or UDP), is defined. It will result in routing the published data from the first network (where the publisher is located) to the predefined domain ID and topic name at the subscriber's network.

The security of the DDS is another unique feature of this standard. It checks the authentication of a participant before initiating the communication procedure to avoid system spoofing by unauthorized agents. Furthermore, an encryption/decryption mechanism, is deployed through a public-private key management mechanism for secure data exchange among participants to avoid data spoofing and bad data injection . Additionally, the security defines a redundant security layer called permission access control, which determines the accessibility for each participant to each domain, topic, and data along with the right to write or read data form that topic. This permission accesses are evaluated through a permission certificate authority, which is initially responsible for signing the certificate for each participant.

\subsection{Proposed Communication Platform}

The proposed decentralized electricity market in Chapter 4 has a data exchange flowchart, which was shown in Figure 4.4. To make this flowchart feasible, we propose a DDS-based communication framework, as shown in Figure 5.1. In this system, each agent is a DDS participant with publisher and subscriber functions. The different publishers and subscribers exchange data through so-called "topics". 


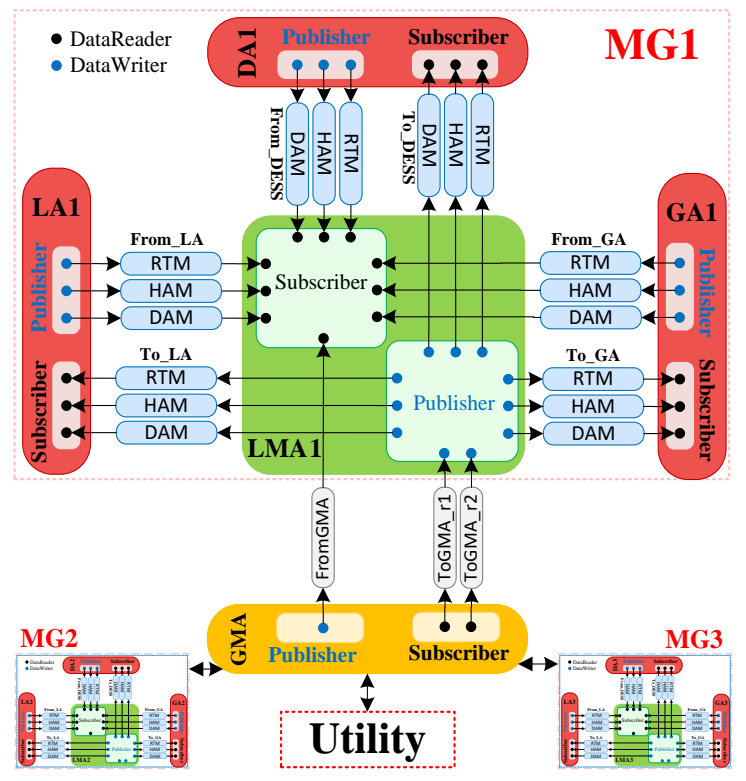

Figure 5.1: Developed communication platform for MAS based hierarchical market structure.

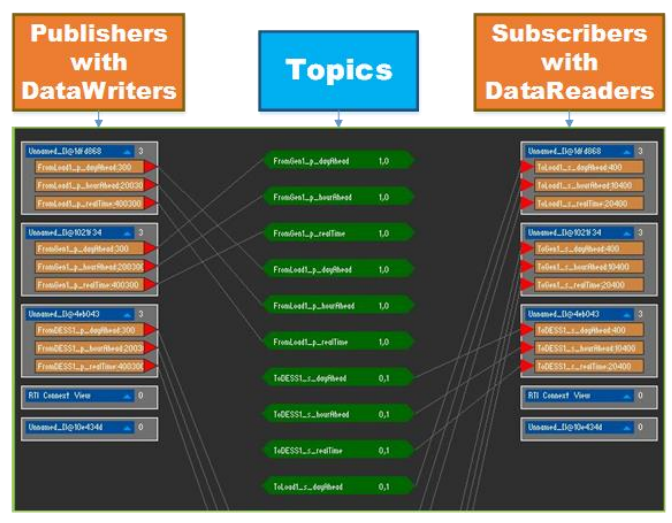

Figure 5.2: Connectivity Visualization of the DDS including Participants and DDS topics within a DDS domain.

Publishers use datawriters to publish data to topics, and subscribers use datareaders to read data from topics on the used domain. Figure 5.2 shows a live tool used to visualize the system connectivity, participants (publishers and subscribers), and topics on the DDS domain [174].

- The blocks on the left-hand side are publishers with their corresponding datawriters.

- The blocks on the right-hand side are subscribers with their corresponding datareaders.

- The green boxes are the topics on the active domain.

- The lines connecting the topics to participants represent the publishing and subscribing processes.

For developing the DDS-based communication system, the communication conditions and requirements for each agent, such as the data-updating frequency, Quality of Service 
QoS, data types, and sequence of events are defined. The data-updating frequency in a multiagent system is controlled by the QoS profile of each agent. The DDS gives too much flexibility in this term by offering a wide range of QoS profiles that could be adapted to meet the different time requirements of the data being sent and received in any application. Another feature of the used DDS is the flexibility it provides in terms of the programming languages and the platforms that can be used to build application systems. In this study, the $\mathrm{C}++$ was used for programming the agent communicating through DDS.

\subsection{Development of the proposed communication platform}

As can be seen in Fig. 5.1, the proposed communication framework for this multiagent system includes a LA, GA, DA, and LMA in each microgrid, with GMA reaching over to all microgrids. In this study, all agents inside a microgrid are simulated on a laptop connected to a different internet network to represent the system that resembles a real wireless environment of a multi-microgrid system, where each agent will be embedded at a remote location. All three LMAs, along with GMA, are simulated on two separate PCs connected to an Ethernet network. As illustrated in Figures 4.4 and 5.1, the sequences of processes are sorted into three phases: DAM, HAM and RTM.

In each step, agents collect the data from loads, DGs, and DESSs in the MG, sort and publish them to appropriate topics, and the LMA subscribes to receive this data. After analysis and running the auction and optimizations in the different levels of the market, as described in Chapter 4, the outputs are published to the corresponding topics in such a way that LA, GA, and DA receive data. In this platform, there are six topics for each market agent. Three topics are used for sending data to the LAM corresponding to the three market levels, and three topics for receiving data from the LAM. For example, the topic 
"From_Load_DAM" is utilized for data which needs to be sent from the load agent to the LMA in the DAM while the topic "To_Load_DAM" is deployed for data which needs to be submitted from the LMA to the load agent after running the auction in this market level. Similar topics are defined for GAs and DAs in each of the DAM, HAM, and RTM levels.

Furthermore, the GMA is represented by an agent to communicate with the LMAs and the system operator in the RTM to receive the utility price and assign the remained mismatch to it after closing the inter-microgrids market. Loads, generators, DESSs, and LMAs, along with their associated topics and way of communication between them, are replicated in each microgrid in the system. It should be noted that the use of the RTPS protocol has dramatically facilitated the scalability of the system, making it easy to expand the network by adding future components.

\subsection{Modified communication platform}

Figure 5.1 demonstrates the developed communication framework for the decentralized market operation of a multi-microgrid system. As can be seen in this figure, each local agent (LA, GA, and DA) writes to three topics (named DAM, HAM, and RTM) and reads from another three topics to communicate with the LMA for three steps of the market. It results in 18 topics for communication between LMA and local agents. Furthermore, the LMA uses three topics for data exchange with the GMA. Although this framework is clear

and works properly, the large number of topics within a DDS domain makes it difficult to change the communication framework in case any change happens to the market structure. For example, if we want to add another data object to the topics for any purpose, it should be almost repeated in all the topics within all microgrids. Therefore, decreasing the number of topics will result in better control and expansion capabilities. 


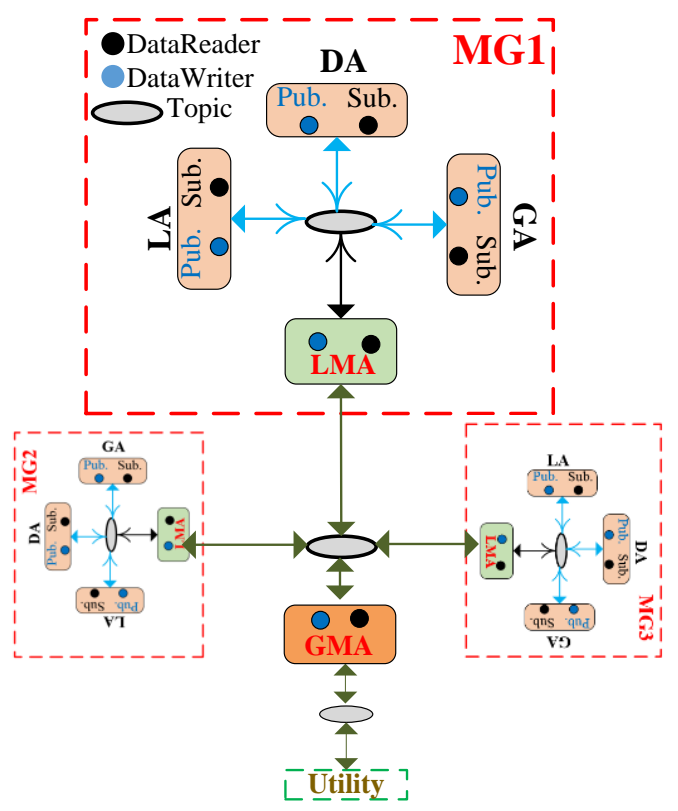

Figure 5.3: The modified communication platform with reduced topics for each MG.

To address these drawbacks, we modify the communication framework, as shown in Fig. 5.3. In this platform, instead of defining 18 topics for communication between LMA and local agents in a microgrid, we can identify just one topic. The same could happen among LMAs and GMA. This reduction is possible by defining proper data objects within each topic through an ID management method. In this method, all local agents and the LMA are simultaneously publish/subscribe to the same topic, and only the ID management scheme determines which topic should write/read or ignore a message. These topics are shown in Figure 5.4 in communicating with local loads and local agents, respectively.

As can be seen in Figure 5.4a, LA uses one topic to communicate with all local loads. In this topic, the first data object is the ID of the participant that determines which agent communicates with the LA. Furthermore, at the bottom of this topic, there are two binary data objects; L_to_A and A_to_L, which are used for a load to agent and agent to load communication orders, respectively. 


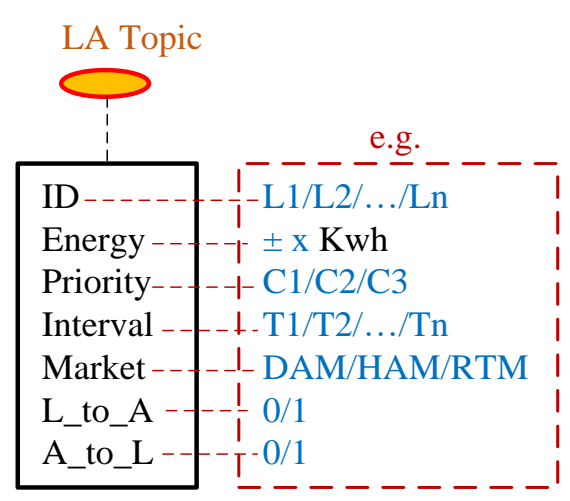

a: LA topic data objects

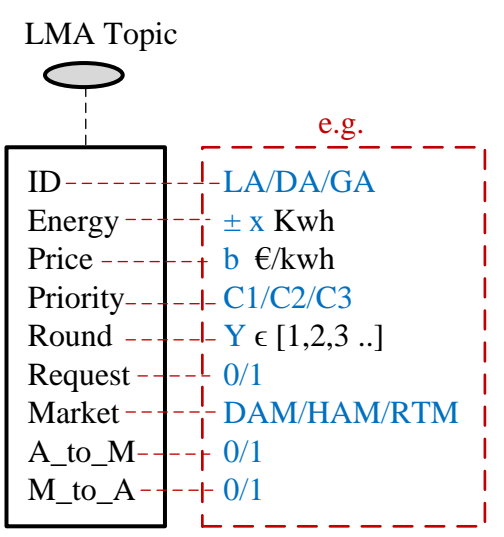

b: LMA topic data objects

Figure 5.4: DDS topics with ID management to optimize the communication framework and reduce the number of topics within a DDS domain.

It means that if a local load wants to send/update its data and inform the LA to read that data, it should write its ID along with all required data on the topic and changes the value of L_to_A from 0 to 1 and vice versa for the A_to_L data object. In this situation, the LA's subscriber, which is continuously read this topic data, finds this information related to the LA, and update related values in its database. Almost the same process happens for communicating between LMA and local agents. Fig. 5.4b shows the ID, read/write data object, the market stage ID (DAM, HAM, RTM), etc. Therefore, the number of topics could decrease significantly, and it helps us to control and protect the system more efficiently.

\subsection{Security of DDS Framework}

The developed communication framework does not include any security algorithm and is open to all possible cyber-attacks. As an example, an attacker could easily monitor the DDS domain, detect the data objects, and publish bad data to that topic. Therefore, to ensure the market players' authentication and correctness of messages, the communication system should be protected by an effective security mechanism. 


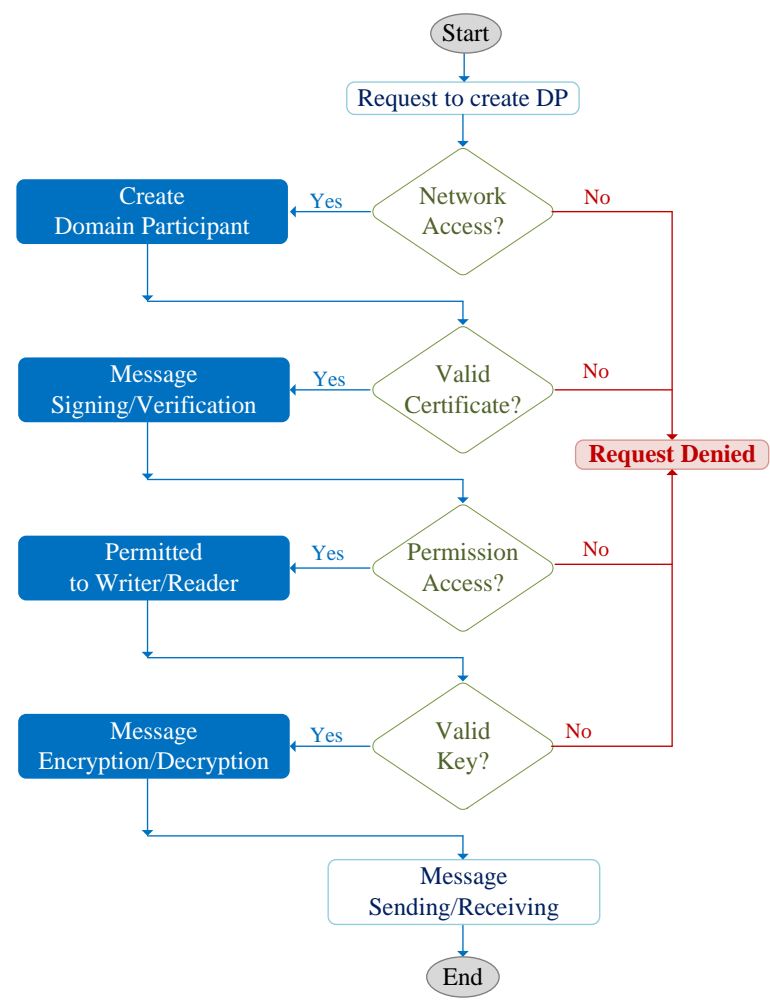

Fig 5.5: DDS security algorithm containing participant authentication, message encryption and permission access definition.

Generally, DDS has its unique security algorithm to authenticate the users, encrypt messages, and check data integrity in the network. Figure 5.5 shows security measures for the DDS including encryption, authentication, and access permission control.

\subsubsection{User authentication}

User authentication is one of the essential steps in secure communication to identify the originality of participants within a protected area. For this aim, a digital signature is used for each authorized participant based on the certified public key. A shared CA must be established within the area, where participants want to communicate. Afterward, each user creates its public key and asks the CA to sign it and register this user. This certificate is used by this participant to sign the messages and communicate with other registered users in this area. 


\subsubsection{Message encryption}

As well as the public key, each participant creates a private key, register it by the CA and use it for message decryption and secure data exchange. In this mechanism, once a user receives a message, checks the identity of the sender through the signed certificate received by message, if a user is authenticated, the receiver deploys its private key to decrypt and read the message.

\subsubsection{Access permission control}

In addition to the security steps mentioned above, there is a redundant security mechanism that explicitly controls the reading/writing actions, called access permission control. This mechanism controls the agents' access to each topic in the DDS domain. It includes joining a DDS Domain, defining a new Topic, reading or writing to/from a DDS Topic, and even reading or writing specific Topic instances. Enforcement of access control shall be supported by cryptographic techniques so that information confidentiality and integrity can be maintained, which in turn requires an infrastructure to manage and distribute the necessary cryptographic keys. The access permission control is defined while the DDS participant is created. It means that we can restrict a participant to write to a topic and another one to just read from a topic or any other profile based on the security requirement. The access permission profile needs to be certified by another CA called the permission certificate authority that signs a permission certificate for each participant. Therefore, for successful communication, users need to use both authentication and permission certificates simultaneously. As it is clear, the permission access control scheme adds a valuable level of security to this system because it makes it very difficult for attackers to get access to secure DDS topics. 


\subsection{DDS security configuration}

DDS security mechanism contains key management by a shared CA and permission access by permission CA, as shown in Figure 5.5. The keys are exchanged using a symmetric encryption algorithm, which is 20 times faster than asymmetric one (which is around $20 \mathrm{~ms})$. Based on numerical results in [175], additional latency due to the security mechanism is highly dependent on the size of the message, implemented unicast/multicast

protocol and speed of the processor. However, the security algorithm can add a latency form couple of microseconds (around 20) to hundreds of us (about 400) to the DDS framework regarding message size, network traffic, processor speed, etc. [176]. Following steps are required to make a DDS framework secure:

- Establishing a local CA.

- Participants create their public and private keys.

- Participants request CA to sign their certificates.

- CA sign the participant certificates.

- Every certificate in the system must be registered with the certificate authority. If a certificate is not found in the CA, the system denies entry.

- Participants use this asymmetric security mechanism to exchange a specific shared key.

- By exchanging the shared key, a symmetric security mechanism is deployed to exchange messages between participants in real-time, where just one agreed shared key is used for both encryption and decryption by all participants.

In fact, the authentication for participants is evaluated through their signed certificates, and they can exchange encrypted data by their public and private keys over the network 
securely. Subsequently, we can control the access of each participant to each topic by defining a permission access security mechanism for the DDS framework. In this mechanism, each participant needs to have specific permissions to get access to different topics as well as the authority to read/write in each of them. The process of establishing this security measure is through the settings of permission and governance files, where access controls are defined. Same as the previous section, and after tuning proper access for each participant, the permission and governance file should be signed by the permission certificate authority and used by the participant to read/write from/to each topic. Therefore, in addition to shared CA, there is a permission CA that signs the permission file for each participant. The domain governance file evaluates this signed permission once the participant wants to communicate with other participants through different topics.

Hence, a participant without a signed permission file cannot get access to these topics in the communication system. It makes the DDS infrastructure more reliable because if an attacker can get access to public and private keys of participants, it will also need the permission file to launch an attack on this system.

\subsection{Security of the Modified Communication Platform}

Figure 5.6 demonstrates the security configuration for the modified communication platform. As can be seen, we have defined several areas to implement the security mechanism based on the structure of the power market. Each region has its own CA and permission CA. For example, a LA is a communication with all loads within a microgrid over a specified secure domain and topic. At the same time, the LA needs to communicate with LMA over another domain. Therefore, it uses another pair of certificates and keys for that purpose. The same process happens for GA and DA. 


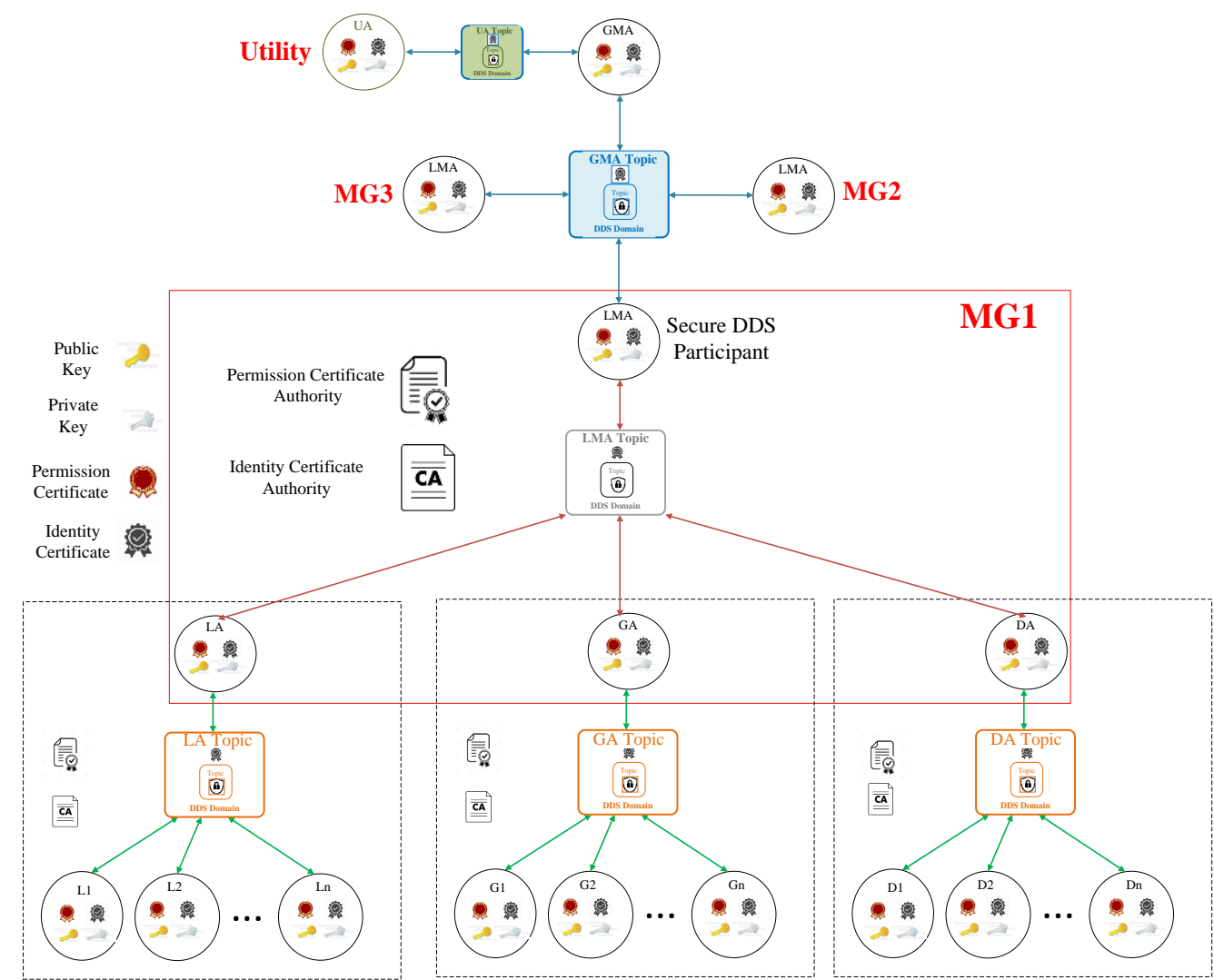

Figure 5.5: Security configurations for the modified communication platform for the MAS-based decentralized electricity market for Multi-Microgrid systems.

In the upper level also LMAs needs to communicate with the GMA over another secure DDS domain. It is worth mentioning that the number of areas with independent CA and key management scheme depends on the structure of the communication system and the level of security, which is needed for that application. Defining more areas means a higher security level. In our application, we defined four secure domains for each microgrid and another safe domain for inter-microgrid transactions

\subsection{Summary}

In this chapter, we developed a data-centric communication framework for data exchange between different market agents for the decentralized market that we developed in Chapter 4. For this purpose, we demonstrated the required data flow between agents within each MG and local market agents as well as communications between MGs and 
general market agents. We modified the developed algorithm by reducing the number of DDS topics to facilitate the flexibility of this framework for any future changes in DDS topic and data objects. We presented the possible cyber threats for the DDS communication platform and investigated about DDS security mechanism to address the user authentication, data encryption and permission access control for different market players within the developed decentralized electricity market. We deployed this communication framework for the hierarchical decentralized electricity market developed in Chapter 4. 


\section{Chapter 6 Hierarchical Blockchain-Based Energy Transaction Framework}

In this chapter, an HBC-based energy transaction framework is introduced for modern power systems, including multi-microgrids in different areas and with high penetration of distributed energy resources, renewable energies, and mobile loads, etc. This framework facilitates the peer-to-peer energy trades between participants using a hierarchical blockchain technology, which significantly enhances the security of the power market, expedites the market clearing process, and considers the power system constraints during the feasibility assessment process. This framework is the combination of two topologies in power markets; Decentralized topology and Blockchain-based topology. It gets benefits from both topologies while avoiding their drawbacks and introduces a modern secure and efficient energy market for advanced power systems.

\subsection{Electricity market models; ros and Cons}

As mentioned in Chapter 3, there is three major power market model. However, each of them has different advantages and disadvantages. In this section, these electricity markets are compared to see the existing challenges and try to address them by developing a new market platform.

\subsubsection{Centralized model}

As mentioned in Chapters 1 and 3, the centralized market is the most conventional framework which is used based on a trusted third-party agent. This platform is easy to use because all members should be registered by the third-party and afterward, by receiving their certificates and security keys, can participate in the market based on the market rules. In this market, all kinds of energy transactions are possible; for example, bilateral trades could happen between participant or market players could actively participate in auction- 
based energy markets. However, there are notable disadvantages of this traditional market model as follow:

1- Centralized control: Using a trusted third-party as control center can cause notable challenges:

- Single point of failure

- High operating costs,

- low transparency

- The potential risk of transaction data modification [177]

2- Users' privacy and security: The third party may monitor users' pattern using historical data and predict their behavior for upcoming market intervals.

3- Cybersecurity issues: This platform is not resilience enough to cyber-attacks.

4- Consumers do not trust the third party: A recent research demonstrate that about $76 \%$ of customers do not trust their utility [178].

5- Big data issue: By increasing in the number of DERs, autonomous MGs, RESs, etc. and their willingness to participate in energy markets, the number of transaction data increased significantly, which cause many problems for gathering, analyzing and clearing the power market.

\subsubsection{Decentralized model}

To overcome the drawbacks mentioned for the centralized market, the decentralized energy market was introduced, which divides the system into several sub-systems and tries to clear the market locally, as described in Chapter 4. The hierarchical structure of this market makes it possible to handle a large number of transactions in proper processing time, increase the data security by breaking the ledger among different agents and address 
the weakness of the single point of failure effectively. However, in this market, individual participation of market players is affected by higher-level agents, which act as aggregators and market agents. In other words, peer-to-peer transactions are just possible in a specific part of the system if the higher-level agents provide the platform for this kind of trades.

\subsubsection{Distributed model}

A highly secure, reliable, and scalable market model is provided by a fully distributed market framework using the BC technology, as described in Chapter 3. In this technology, there is no trusted third-party to evaluate transactions, running the auction, or act as an aggregator for individual participants. In other words, each market player could actively trade energy with other participants. All agents validate transactions in the system which have access to all previous transactions in the system through their highly secure and synchronized ledgers.

Despite the advantages of this technology, there are any challenges for the BC-based electricity markets as follow [179]:

1- Low efficiency: The transaction speed in the BC technology cannot meet the system requirements due to the structure of this framework.

2- Scalability: By increasing the number of participants in large-scale networks, a massive amount of trades and transaction data must be recorded in the $\mathrm{BC}$ databases.

3- High transaction costs: It can raise the overall system cost because participants do not ensure that social welfare is maximized.

4- Privacy issue: Since transaction data are available for all market participants, the privacy of participates is a significant challenge. An agent may analyses data to 
extract the behavior models and can predict other agents' actions in the market. It is also a reason for the lack of motivation for participants.

5- Communication issues: $\mathrm{BC}$ technology needs a considerable amount of real-time sensor and market data communication.

6- Energy price: Although $\mathrm{P} 2 \mathrm{P}$ transactions are possible, reaching an agreement for energy prices in the real-time market is an issue.

7- High energy consumption: The $\mathrm{BC}$ miners consume too much energy for the mining process.

8- Hardware limitation: Each agent in the market needs new hardware setup as well as well-developed software to participate in the market and perform smart contracts in coordination with the energy management system.

9- Cybersecurity issues: Although the BC technology structure makes it very robust against cyber-attacks, this system is not still 100\% secure. For example, five attack models were introduced for the BC-based systems in [177]. Furthermore, there is always the possibility of leaking private keys information [180].

10- Transaction feasibility assessment: For a transaction to be feasible in a power system, not only availability of energy and financial aspects must be verified, the possibility of each trade should be assessed by the system operator to prevent any violation in power system constraints. In BC technology, because of the lack of trusted-third party or local agents, this assessment is almost impossible. In fact, this is a critical challenge once the BC technology is used for electricity 
transactions, mainly when an independent and constrained transmission system is used to transfer energy between participants.

The BC technology is still new, and many research and experiments are being conducted to address the challenges mentioned earlier [179]. For example, improving the consensus mechanism, transaction processing speed, and energy consumption rate by and introducing PoS or PBFT instead of PoW. Deploying advanced communication standards for real-time applications, passing new regulatory laws for energy price, and creating incentive programs to motivate participants in using this technology.

However, the transaction feasibility assessment issue has not been studied in the literature, a topic that is essential in electricity markets to guarantee the secure operation of the power systems. Therefore, in this chapter, we introduce a HBC-based energy transaction framework which not only addresses the feasibility assessment issue but also improves the scalability of the BC networks, transaction processing time, and participants' privacy concerns. Table 6.1. Summaries all pro and con mentioned above for three energy market models as well as developed HBC model, which will be described in the next section.

Table 6.1: Comparison of energy transaction frameworks for the centralized, decentralized, distributed and hybrid market models.

\begin{tabular}{|c|c|c|c|c|}
\hline Market Framework & Centralized & Decentralized & Distributed & developed HBC \\
\hline Efficiency & $\checkmark$ & $\checkmark$ & $\bigcirc$ & $\bigcirc$ \\
\hline Scalability & O & $\checkmark$ & $x$ & $\checkmark$ \\
\hline Transparency & $x$ & O & $\checkmark$ & $\checkmark$ \\
\hline Cybersecurity level & 区 & $\bigcirc$ & $\square$ & $\square$ \\
\hline Participants' Privacy & $\nabla$ & O & $x$ & O \\
\hline Low Transaction costs & $\bigcirc$ & O & $\mathrm{x}$ & $\bigcirc$ \\
\hline Minimum Energy Cost & $\square$ & O & 区 & O \\
\hline Feasibility assessment & $\nabla$ & $\checkmark$ & $x$ & $\checkmark$ \\
\hline Development & $\checkmark$ & $\bigcirc$ & $x$ & $x$ \\
\hline \multicolumn{5}{|c|}{ Legend: $\checkmark$ : Acceptable Performance } \\
\hline
\end{tabular}




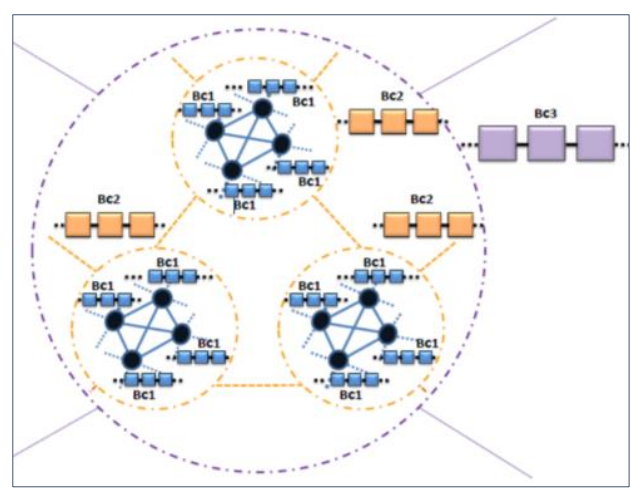

(a)

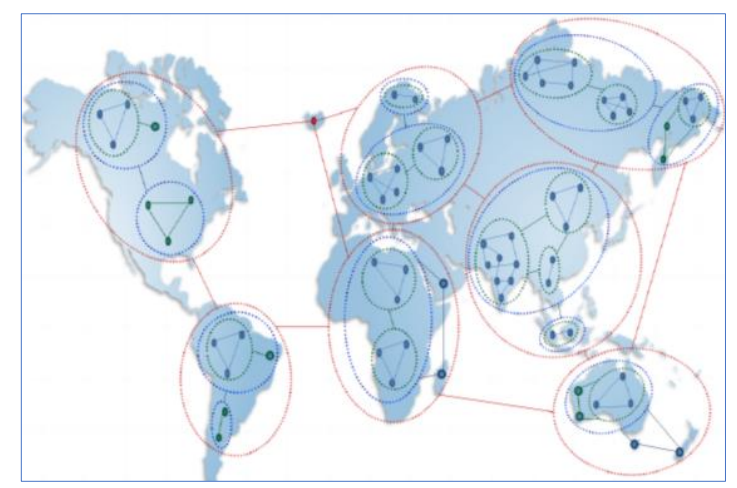

(b)

Figure 6.1: Hierarchical blockchain model developed by S. Sahoo et al. [181]

\subsection{Hierarchical Blockchain-based Market Model}

The concept of the hierarchical blockchain for large-scale IoT applications was introduced by Swagatika et al. to address the scalability of the $\mathrm{BC}$ with a notable number of transaction data [181]. Figure 6.1a demonstrates the hierarchical BC model containing local BCs to record local transactions and higher-level BC, which determines different local networks with abstracted transaction records. This model fits large size networks with a large number of participants usually spared in a wide geographical area along with too many transaction records, as shown in Figure 6.1b. Defining sub-networks and abstraction method depends on the application and requirements as were discussed in detail in [181]. An example of these systems is a multinational insurance company that is expanded over many countries around the world. Using hierarchical BC is an effective method to avoid providing extra information, which is not required for some evaluations, where each level presents various data formats. For example, a police officer may want to know the driver has insurance or not? Definitely, the insurance information is not essential at this level, and the abstraction model can be the right solution for this problem. Moreover, the results in [181] show a significant reduction in data-size (around 75\%) when the hierarchical BC with local networks is deployed comparing with a concrete $\mathrm{BC}$ including all data. 


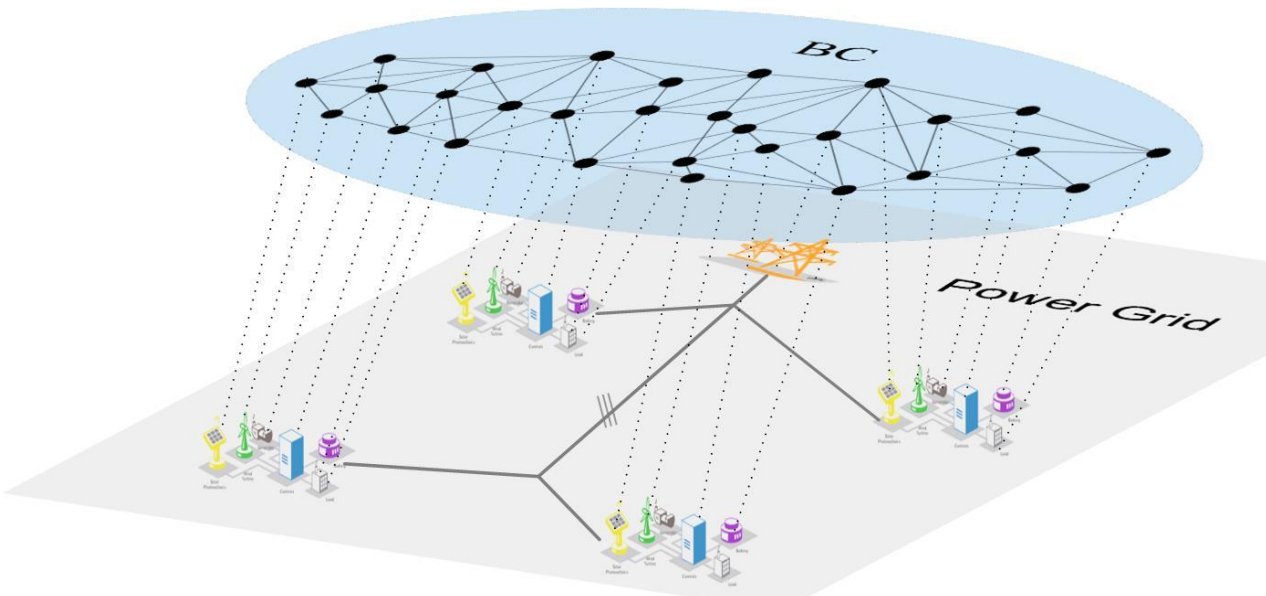

Figure 6.2: Conventional blockchain-based energy transaction framework with all agents in a single BC.

\subsubsection{The proposed HBC model for multi-microgrid systems}

Inspired from these facts, we propose an $\mathrm{HBC}$ technology for energy trades in modern power systems with high penetration of individual market players, including renewable energy resources, distributed generations, electric vehicles, and autonomous microgrids. The HBC technology is a combination of decentralized and distributed energy transaction frameworks with a unique structure that addresses security concerns while provides open access, reliable and scalable power market framework, as shown in Table 6.1. It can be seen that the HBC technology benefits from positive features of both decentralized and distributed structures while it does not have their drawbacks. Figure 6.2 shows the structure of conventional blockchain technology for energy transactions in an area with several microgrids. This structure defines an agent for each participant in the market. By joining the market, the agent immediately receives a copy of the ledger containing all previous transactions in the systems. 


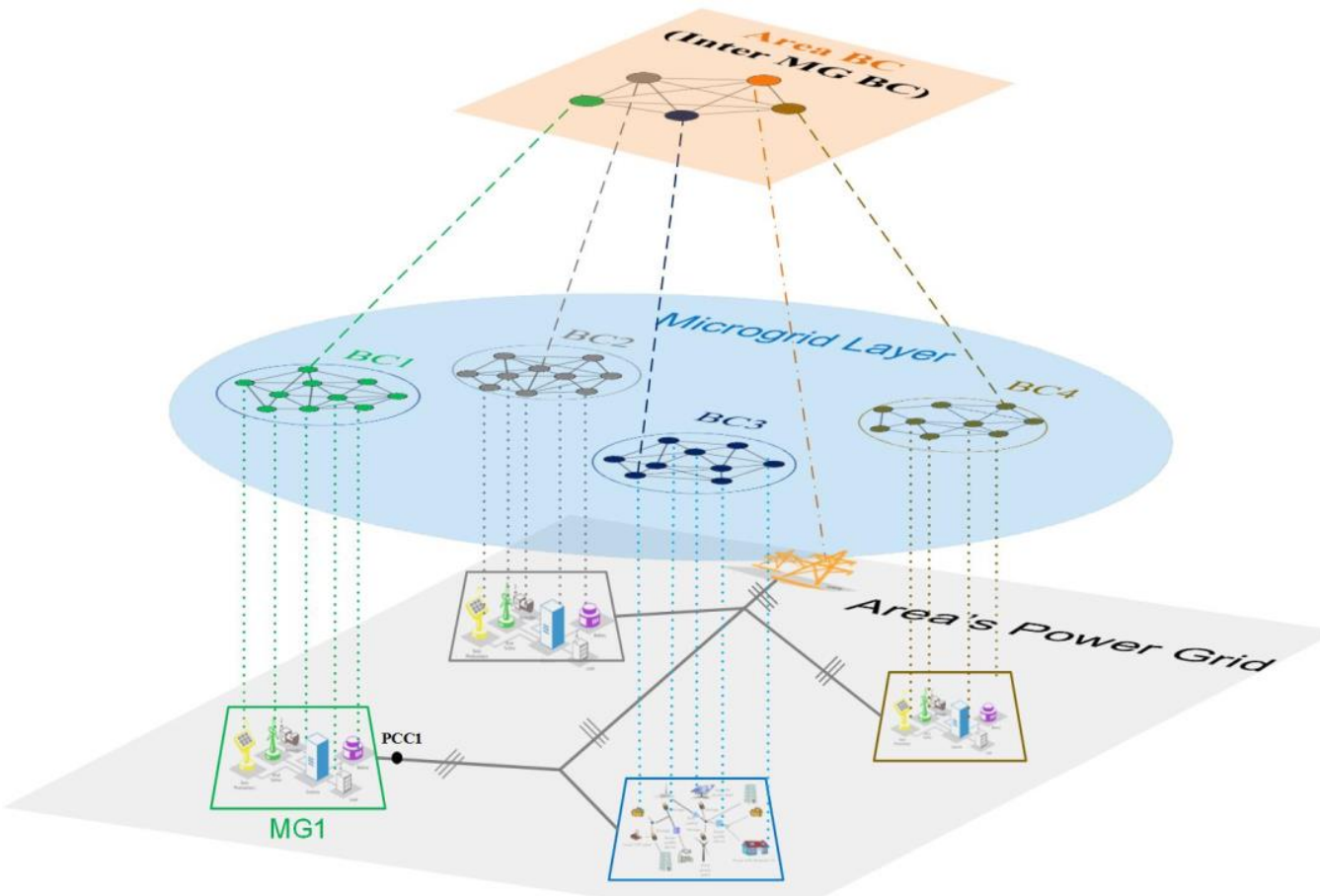

Figure 6.3: The proposed HBC energy transaction framework for a Multi-MG system including two layers for energy transactions.

As mentioned before, besides all drawbacks of BC technology for energy transactions (such as latency in data processing and mining, privacy, etc.), the main concern is about the feasibility of energy transactions from a power system point of view. It means that, for example, the system might experience a voltage drop or transmission line congestion due to an inter-microgrid transaction. To overcome all these drawbacks and consider the power system constraints to verify the transactions' feasibility, we propose the HBC technology, as shown in Figure 6.3. The HBC is a combined technology of decentralized and distributed frameworks; it includes independent $\mathrm{BC}$ for each microgrid while a higher-level $\mathrm{BC}$ is used for inter-microgrid transactions.

\subsubsection{Model description}

To clarify the HBC technology in detail, we consider a multi-microgrid area, including several microgrids connected through the distribution network and a connection point to 
the utility grid. As can be seen in Figure 6.3, there are three layers in this platform; Area's Power Grid layer, Microgrid layer, and Inter-Microgrid Layer.

\subsubsection{Area's power grid layer}

The Area's power grid layer is the physical power network layer containing several microgrids connected through the distribution network and a connection point to the utility grid. The power network configuration such as transmission lines, transformers and location and elements of each microgrid are shown in this layer.

\subsubsection{Microgrid layer}

The second layer of this platform demonstrates the Microgrid layer, including several independent BCs. Each BC in this layer is related to one of the microgrids in this area; for example, the $\mathrm{BC} 1$ is a blockchain network for the Microgrid1 (MG1), where all components within this microgrid can do the peer-to-peer transactions using a conventional $\mathrm{BC}$ technology. Since the transactions within the microgrid do not affect the distribution network, only feasibility of trades is evaluated to verify the available energy for prosumers.

\subsubsection{Inter-Microgrid layer}

The third layer of this market platform is the Inter-Microgrid Layer, which is designed to handle energy transactions among different MGs in this area. Once independent BCs in the Microgrid layer finalize the last block of transitions in each Microgrid, all the BC agents in that Microgrid would have a similar ledger, including all verified energy trades. In this condition, if there is still potential for energy trades, the inter-MG market mechanism should be activated. This could happen if the expectations of an agent within this MG is not satisfied by other local BC agents. For example, an agent has more energy to sell, but there is no more interest or need for this energy in the local MG or price is not tempting 
for local buyers. On the other side, and probably several agents within each MG are in the same condition for producing or consuming more energy by their desired price and willing to do the transaction with other agents in other MGs.

However, for P2P inter-microgrid transactions using the $\mathrm{BC}$ technology, besides, to check the energy availability for each transaction, the power system constraints must be evaluated by participants to meet the operational limits of the power system. The power system constraints for inter-MG transactions are presented by (6-1)-(6-4). It includes checking the transmission lines capacities, voltage magnitude, transformers' loading and the amount of energy exchange with the utility grid.

$$
\begin{gathered}
V_{i}^{\text {min }} \leq\left|V_{i}^{j}\right| \leq V_{i}^{\text {max }}, \mathrm{j}=\mathrm{a}, \mathrm{b}, \mathrm{c}, \mathrm{i}=1,2,3, \ldots, n_{\text {bus }} \\
I_{j}^{k} \leq I_{j}^{\text {max }}, \mathrm{j}=\mathrm{a}, \mathrm{b}, \mathrm{c}, \mathrm{k}=1,2,3, \ldots n_{\text {line }} \\
S_{j}^{T} \leq S_{j}^{\text {max }}, \mathrm{j}=\mathrm{a}, \mathrm{b}, \mathrm{c}, \mathrm{T}=1,2,3, \ldots, n_{\text {Transformer }} \\
S_{\text {Utility }}^{\text {min }} \leq S_{\text {Utility }} \leq S_{\text {Utility }}^{\max }
\end{gathered}
$$

However, for checking the above-mentioned operational constraints by all participants in the network, they need to run power flow calculations based on an updated model of the power grid and a computational tool (e.g., power system software), which is practically impossible. The proposed HBC address this problem by introducing the equivalent model of Microgrids in the Inter-MG layer.

\subsubsection{HBC model development}

The proposed HBC model is developed through the following steps:

1. Each entity in the physical layer (Power Grid layer) is linked to a BC agent in the MG layer and trade energy with other components in the same MG. 
2. Each MG in the MG layer has an agent who works on its behalf in the Inter-MG layer to trade energy with other MGs.

3. The utility grid also has its agent in the Inter-MG layer, demonstrating the available energy support from the utility.

4. Upon receiving the inter-MG transaction request, the agents in the Inter-MG layer randomly choose one of the agents in their local BC and get a copy of the ledger.

5. Since this ledger contains all transaction records of that $\mathrm{MG}$, the potential of the inter-MG transaction could be obtained by summarizing the ledger, as shown in Table 6.2 and Figure 6.4.

6. Each agent in the Inter-MG layer has an equivalent model of the power system along with its own MG, as shown in Figure 6.5.

7. For each transaction between MGs, the energy availability and transaction feasibility are checked before approving it.

8. Each agent in the Inter-MG layer updates the ledger of its local BC by sending the ledger to at least $51 \%$ of the agents in the local BC.

9. The agents evaluate the received updates and synchronize their ledgers accordingly. The two critical aspects of this platform are the ledger summarization and the power grid equivalent model, which are described in this section.

Table 6.2: Local and summarized ledger of BC1.

\begin{tabular}{|c|c|c|c|c|c|c|c|c|c|}
\hline \multicolumn{5}{|c|}{ Ledger data in $\mathrm{BC} 1$} & \multicolumn{5}{|c|}{ Summarizing the ledger of BC1 } \\
\hline Transaction \# & From & To & Energy & Price & A1 & $\mathrm{A} 2$ & A3 & A4 & A4 \\
\hline 1 & A1 & A2 & $\mathrm{E} 1$ & $\mathrm{P} 1$ & $-\mathrm{E} 1$ & $+\mathrm{E} 1$ & 0 & 0 & 0 \\
\hline 2 & A1 & A3 & E2 & $\mathrm{P} 2$ & $-\mathrm{E} 2$ & 0 & $+\mathrm{E} 2$ & 0 & 0 \\
\hline 3 & A4 & A2 & E3 & P3 & 0 & $+\mathrm{E} 3$ & 0 & -E3 & 0 \\
\hline$\ldots$ & $\ldots$ & $\ldots$ & $\ldots$ & $\ldots$ & $\ldots$ & $\ldots$ & $\ldots$ & $\ldots$ & $\ldots$ \\
\hline $\mathrm{n}$ & A5 & A3 & En & $\mathrm{Pn}$ & 0 & 0 & $+\mathrm{En}$ & 0 & -En \\
\hline \multicolumn{5}{|c|}{ Available Energy: $A_{k}^{A v a}=A_{k}^{\max }-\sum_{i=1}^{n} A_{k}^{i}$} & $A_{1}^{A v a}$ & $A_{2}^{A v a}$ & $A_{3}^{A v a}$ & $A_{4}^{A v a}$ & $A_{5}^{A v a}$ \\
\hline \multicolumn{5}{|c|}{ Bid } & b1 & $\mathrm{b} 2$ & b3 & $\mathrm{b} 4$ & b5 \\
\hline
\end{tabular}




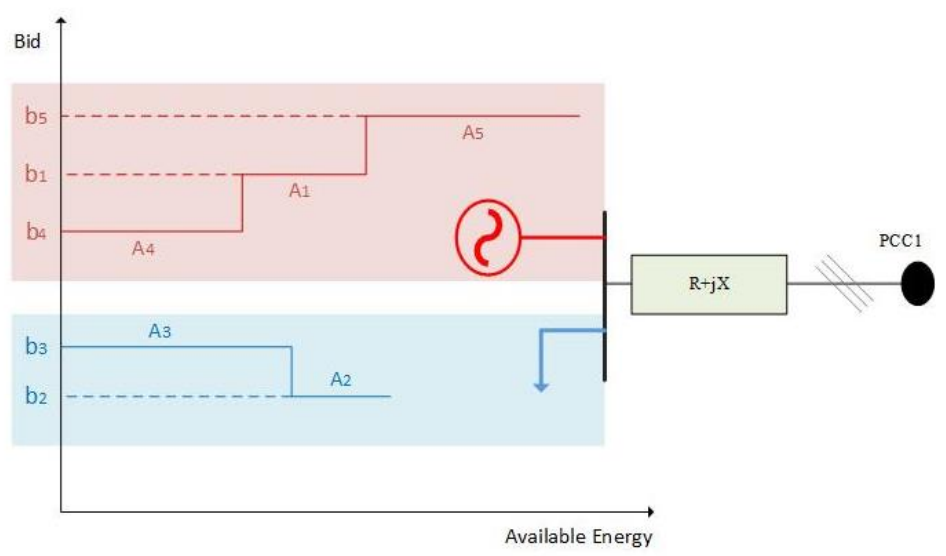

Figure 6.4: Energy cost functions in equivalent model of MG1 in the HBC model.

\subsubsection{Summarizing the local BC ledger}

Table 6.2 reports a detailed analysis of the $\mathrm{BC} 1$ with five agents as an example and shows the ledger summarization process. The available energy for the inter-MG transaction by each agent in local $\mathrm{BC}$ is calculated by the Inter-MG layer agent considering the maximum available energy of that agent and all approved transactions in the local BC. This summarization results in the energy cost functions for the generator and load within the microgrid which are used for the peer-to-peer transaction with other MGs.

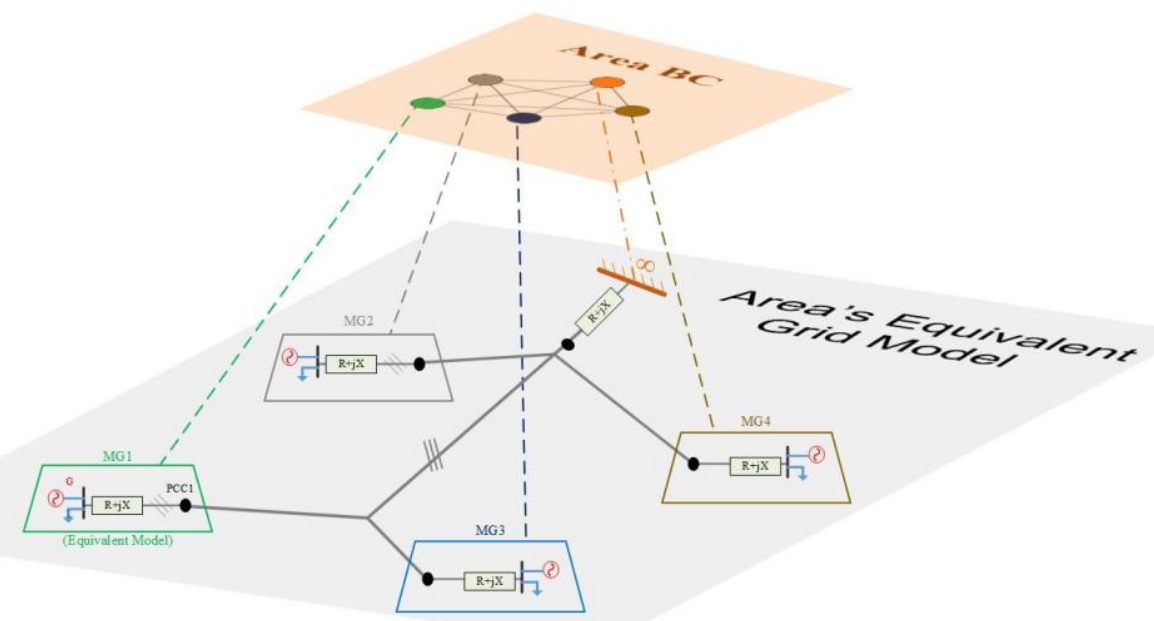

Figure 6.5: Area's equivalent grid model for inter-microgrid transactions 


\subsubsection{Power grid equivalent model}

Figure 6.5 shows the equivalent system model used by agents in the Inter-MG. Each MG is modeled by a generator, a load, and an impedance. The values for impedance are calculated based on the impedance of transmission lines or transformers, which connects the MG to the distribution network. The cost functions for MG's generation and load potentials are obtained from ledger summarization process.

\subsection{Numerical results}

We developed the proposed HBC platform using Java programing language within Eclipse text editor as an IDE. Before presenting numerical results and discussions, a brief description of this algorithm is presented in this section.

\subsubsection{Program description}

We applied the SHA256 cryptographic algorithm for digital signature and calculating the Hash during the mining process. The program is initiated by preparing a wallet for each participant containing the available energy/demand obtained from local BC ledger summarization. Each participant afterward needs its public and private key to trade energy within the BC network actively. The public key is shared with all other participants while the private key is kept secret and is used to sign transactions.

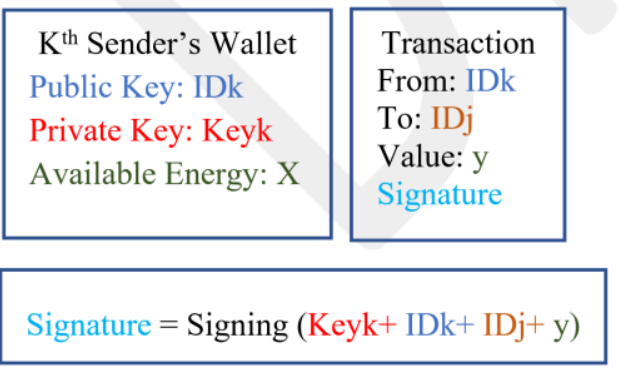

Verification
$\quad(\mathrm{IDk}+\mathrm{IDj}+\mathrm{y})=$ ? Signing (IDk + Signature)
If No, Deny the transaction
If Yes, is Transaction approved for availability
and Feasibility?
If NO, Deny the transaction
Otherwise, add the transaction to the Block

Figure 6.6: Transaction structure and verification process in the BC technology. 
Each transaction must be approved by majority of participants in the network. A transaction consists of public keys of sender and receiver and the energy value. All this data is signed by the sender using its private key. The transaction data along with the signature is then published to the network for validation. Other entities use the senders public key to verify the signature and confirm the senders authentication. Once it is approved, they evaluate the possibility of energy trade considering the available energy for the sender as well as feasibility of transactions from power system point of view by calculating power flow in equivalent system model. All these processes are shown in Figure 6.6. By evaluating all received transactions regarding their receiving time, they are added to a new block. This block must be mined before it can be linked to blockchain. For mining process, the miner needs the Hash of previous block, package of approved transactions and a timestamp to begin the mining process to find a nonce which can meet the pre-defined Hash difficulty. Once the block is mined (As shown in Figure 3.2), it is added to the existing chain of block.

\subsubsection{Case study}

The case study is the multi-microgrid system presented in Chapter 4 containing three MGs, as shown in Figure 6.7a. Three independent MGs have their BC for transactions within the MGs. For Inter-MG energy trades, an equivalent model is needed regarding the summarized ledger of each local BC which is shown in Figure 6.7b.

\subsubsection{Power system constraints}

In Chapter 4, section 4.4.3, the inter-MG market was cleared by a reverse auction model. In that scenario, the MG1 sold $32 \mathrm{KWh}$ energy to the MG2 and MG3 to keep the balance of the generation and load within the whole area at the 9th RTM market interval. 


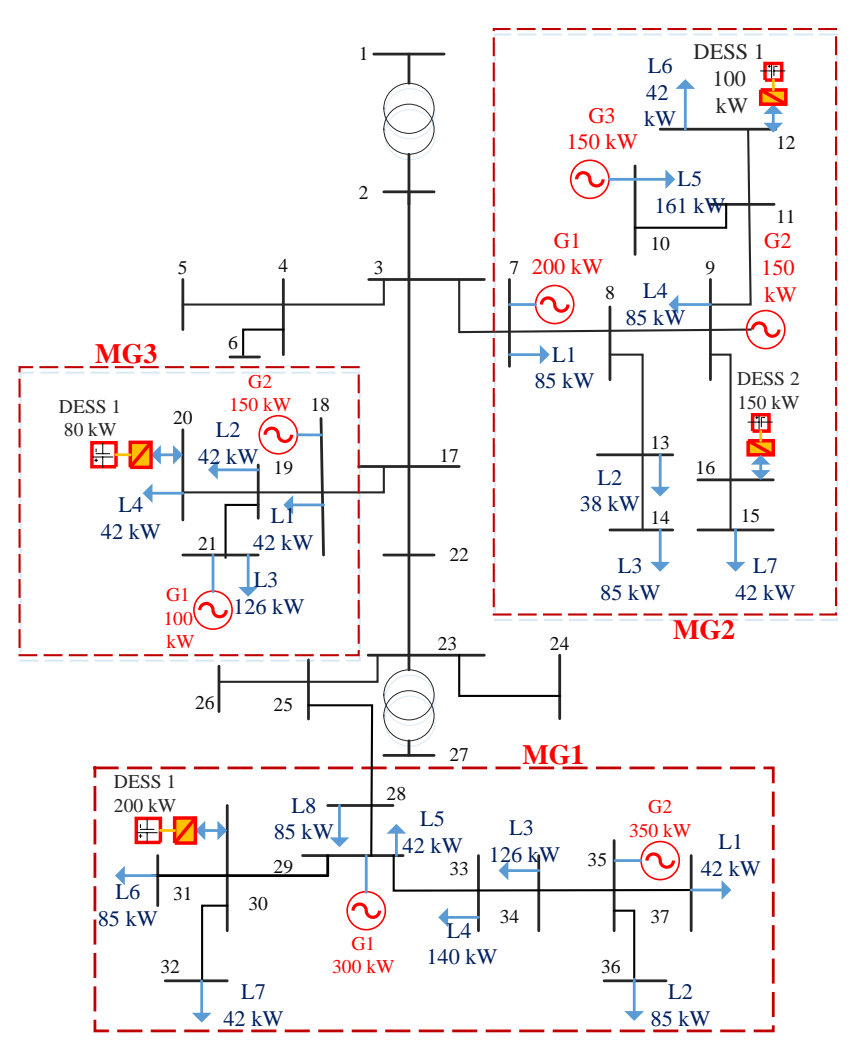

a) Microgrid layer model

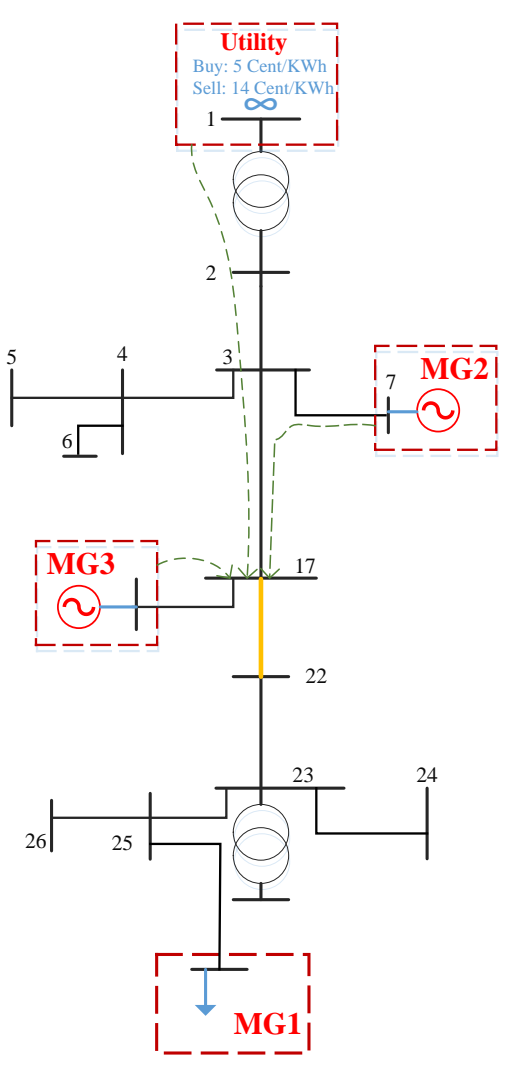

b) Inter-MG Layer model

Figure 6.7: Power grid and equivalent model for BC-based transactions.

The actual active power flow for this transaction should pass over the Lines 17-22, 2223, 23-25, and 25-28, which connects the MG1 to the local distribution network. In that case study, the system did not experience and congestion over the distribution network for inter-Microgrid energy trades. In order to show the effect of power transmission constraints on power market transactions, we assume that the MG1 loses a significant portion of its generation capacity due to maintenance of the DESS1 and fault on G1 (Losing $500 \mathrm{KW}$ of its generation capacity).

Therefore, by considering $647 \mathrm{KW}$ of load demand and $350 \mathrm{KW}$ of G2's generation, the MG1 needs to buy $397 \mathrm{KWh}$ to feed its loads. In fact, it is the value of the summarized ledger of MG1. The specific demand cost function is shown in Figure 6.8a. The generation 
capacity and cost fiction for MG2 and MG3 are also shown by Figure $6.8 \mathrm{~b}$ and c, respectively. The configuration of the network shows that regardless of the energy sellers' location for this transaction, the active power flow should pass the Line 17-22, which is a three-phase 2/0 AA, CN cable with a thermal capacity of 175 AMP [182]. Since the 37bus system is a highly unbalanced system, power flow studies show that before MG1 lose its generation capacity, the three-phase loading was 147.60, 99.37, and 106.19 AMP, respectively. It means that the loading of phases $\mathrm{A}, \mathrm{B}$, and $\mathrm{C}$ is $84.3,56.7$, and $60.68 \%$, respectively. Considering the 0.85 as the power factor, the free capacity of phase $\mathrm{A}$ is around $65.67 \mathrm{KW}$. This value is around 181.5 for phase B and $165.14 \mathrm{KW}$ for phase C. It can be concluded that the total free capacity of this corridor is $412.314 \mathrm{KW}$, which is more than the total demand for this transaction $(397 \mathrm{KW})$. However, the phase A can carry up to 65.67 KW for this transaction. Thus, the possibility of this transaction depends on the demand and generation scheme. For example, if it is a balanced three-phase demand, each

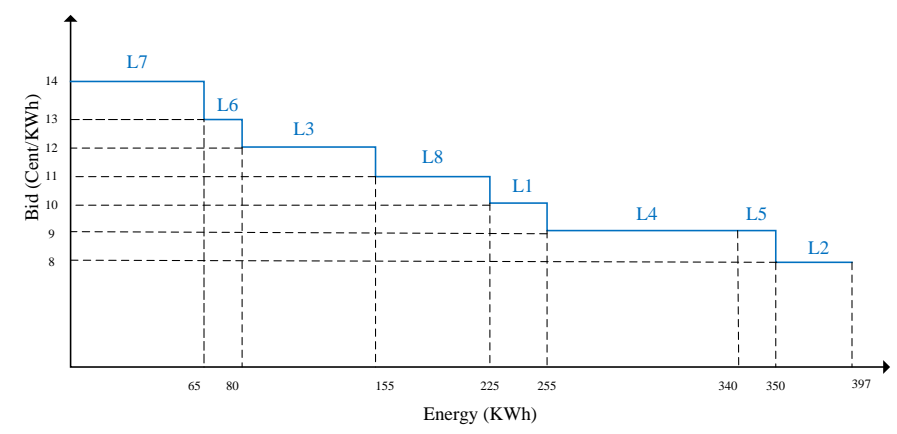

a) Energy demand function for MG1

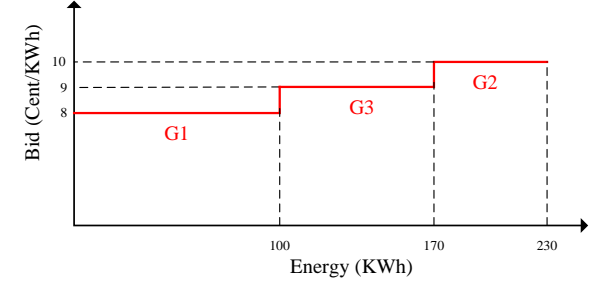

b) Generation cost function for MG2

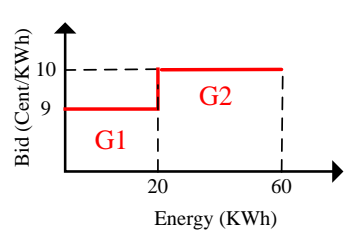

C) Generation cost function for MG3 Figure 6.8: Summarized ledgers for agents in the Inter-MG layer for energy transactions between MGs. 
phase needs around $132 \mathrm{KW}$. The phase A maximum free capacity is 65.67. Therefore, only $197.1 \mathrm{KW}(49.62 \%)$ of the demand can be supplied without violating the power system constraints. It is worth mentioning that the three-phase unbalancing problem is a control issue that can be addressed by optimal power routing schemes. This topic will be discussed in the next chapter of this dissertation.

\subsubsection{Inter-MG transactions}

The summarized ledgers, energy cost functions, and equivalent network models were developed in the previous sub-section. Now the HBC is run to clear the inter-MG transactions. Table 6.3 reports the transaction requests by market agents within the interMG layer of the HBC. It shows six trade requests. Although the BC approves all six transactions for energy availability, only the first three of them meet power system constraints. By rejecting transactions number 4,5 and 6, the MG2 evaluates the network capabilities and reduce its transaction request from 60 to $7 \mathrm{KW}$. Other agents also retry to do more transactions, as shown in block 3 of Table 6.3 .

\begin{tabular}{ccccccccc}
\multicolumn{8}{c}{ Table 6.3: Transaction requests for inter-MG market. } \\
Block & Order & From & To & Energy & Price & Energy availability & Feasibility & Add to block \\
& 1 & MG2 & MG1 & 100 & 8 & $\square$ & $\square$ & $\square$ \\
& 2 & MG3 & MG1 & 20 & 9 & $\square$ & $\square$ & $\square$ \\
2 & 3 & MG2 & MG1 & 70 & 9 & $\square$ & $\square$ & $\square$ \\
& 4 & MG2 & MG1 & 60 & 10 & $\square$ & $\square$ & $\square$ \\
& 5 & MG3 & MG1 & 40 & 10 & $\square$ & $\square$ & $\square$ \\
& 6 & Utility & MG1 & 107 & 14 & $\square$ & $\square$ & $\square$ \\
3 & 1 & MG2 & MG1 & 07 & 10 & $\square$ & $\square$ & $\square$ \\
& 2 & MG3 & MG1 & 10 & 10 & $\square$ & $\square$ & $\square$ \\
& 3 & Utility & MG1 & 20 & 14 & $\square$ & $\square$ & $\square$
\end{tabular}

Table 6.4: Final ledger for inter-MG blockchain.

$\begin{array}{ccccc}\text { Order } & \text { From } & \text { To } & \text { Energy } & \text { Price } \\ 1 & \text { MG2 } & \text { MG1 } & 100 & 8 \\ 2 & \text { MG3 } & \text { MG1 } & 20 & 9 \\ 3 & \text { MG2 } & \text { MG1 } & 70 & 9 \\ 4 & \text { MG2 } & \text { MG1 } & 07 & 10\end{array}$


This transaction is then sent to the network and gets approved by the other agents in the system. The final ledger for the inter-MG market is shown in Table 6.4. Figure 6.9 shows the results in the Eclipse console and all steps to finalize transactions within the inter-MG layer. In Figure 6.10, the results are shown for a $\mathrm{BC}$ without considering the power system constraint during the transaction verification process, which approves all transactions in the second block. However, it is not feasible in real power system operation, and the line 17-22 will be tripped due to overloading. Once the inter-MG market is cleared, agents should update the ledger of their local BCs, and send the updated ledger randomly to at least $51 \%$ of the agents within the MG layer. The additional blocks for MG1, 2, and 3 are shown in Table 6.5. For example, in MG1, the total demand was $397 \mathrm{KWh}$ for this market interval.

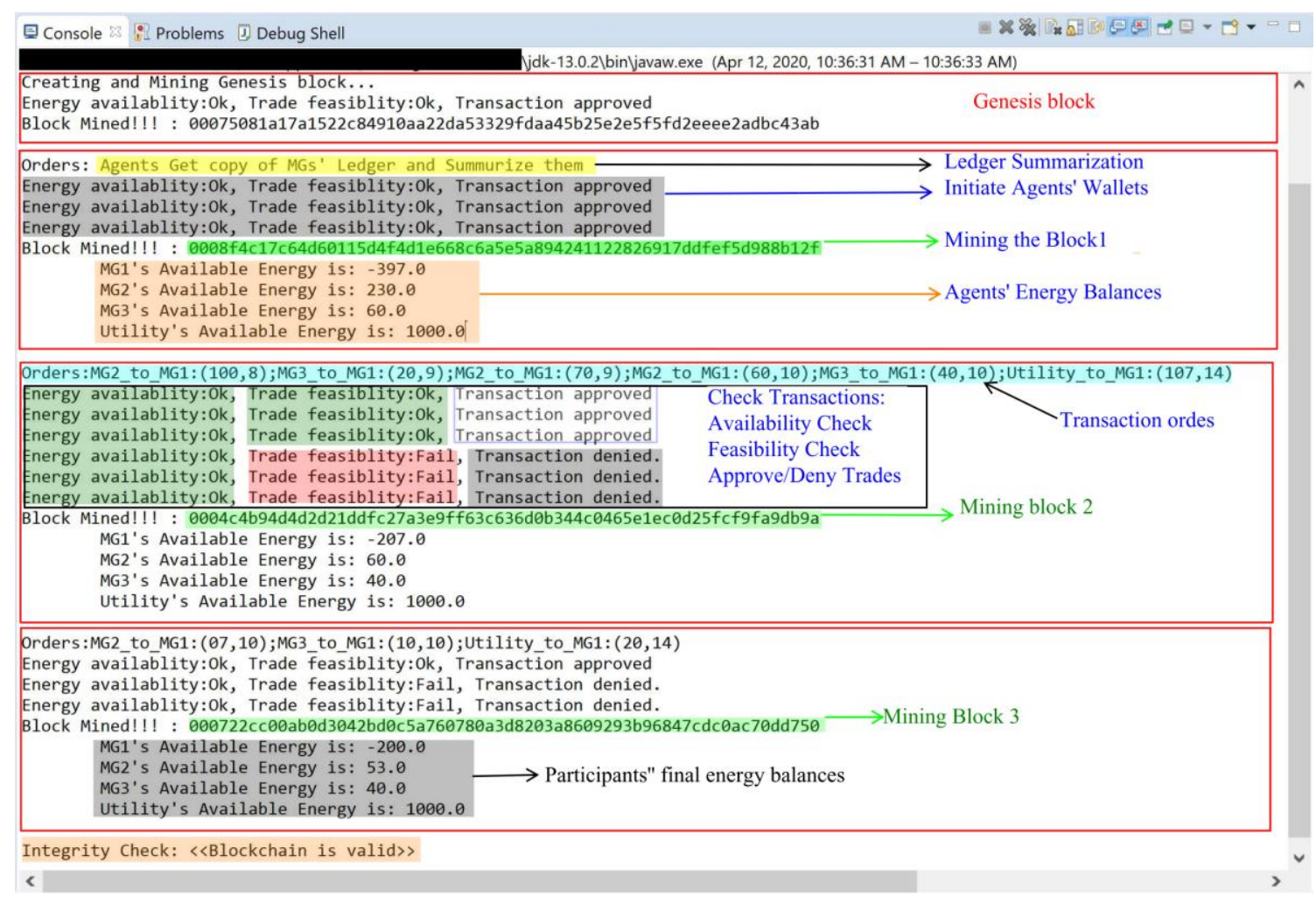

Figure 6.9: Eclipse console view for the HBC results considering the power system constraints before approving each transaction. 


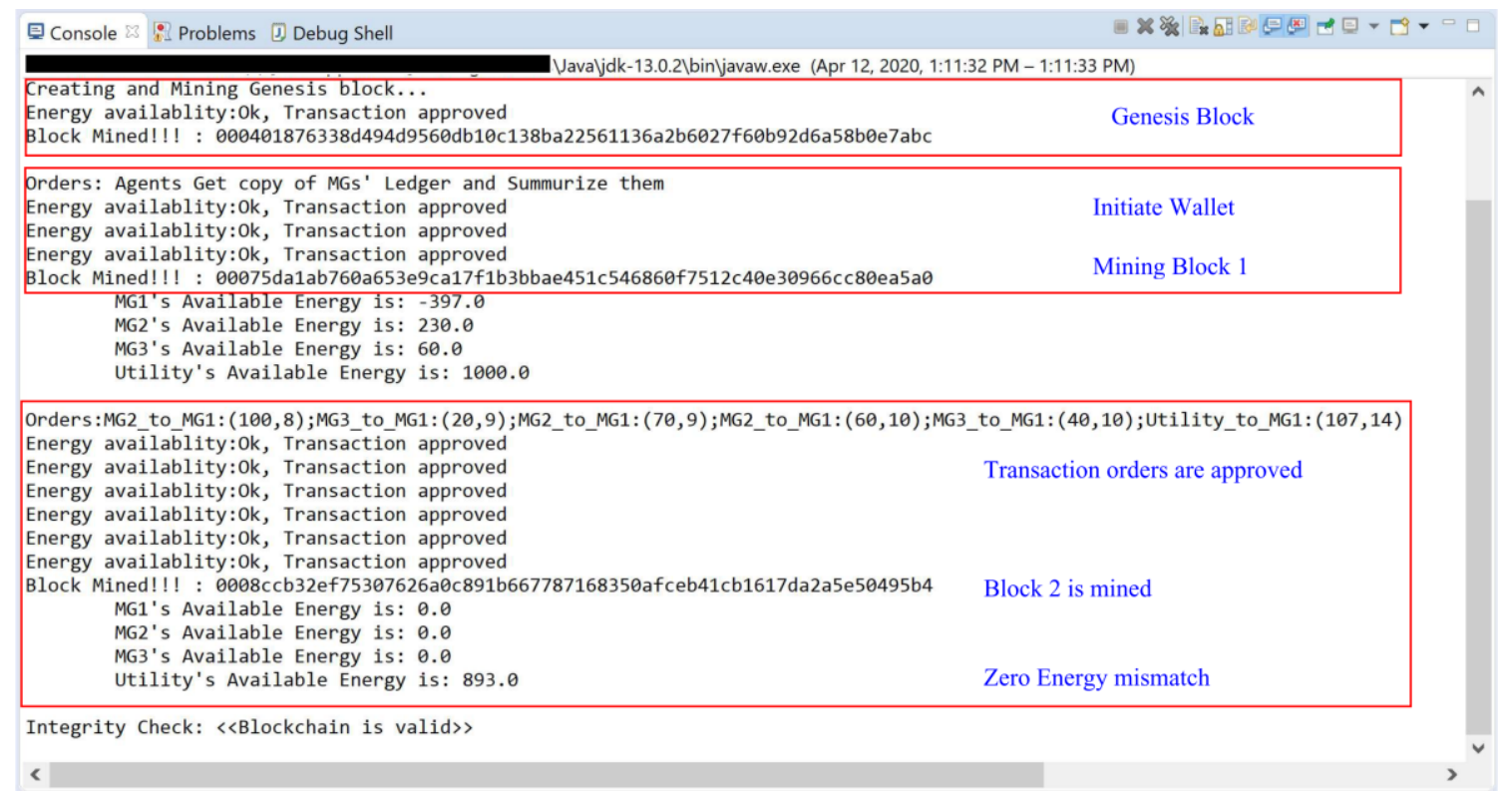

Figure 6.10: Eclipse console view for the HBC without considering the power system constraints in transaction approval process.

Table 6.5: Additional blocks to update the local BCs ledgers.

\begin{tabular}{ccccccccccc} 
Order & \multirow{2}{*}{ From } & MG1 & To & Energy & Price & Order & From & To & Energy & Price \\
1 & & L7 & 07 & 10 & 1 & G2 & & 07 & 10 \\
2 & & L7 & 58 & 9 & 2 & G3 & Local Grid & 70 & 9 \\
3 & \multirow{2}{*}{ Local Grid } & L6 & 15 & 9 & 3 & G1 & & 100 & 8 \\
4 & & L3 & 17 & 9 & & & MG3 & & \\
5 & & L3 & 58 & 8 & Order & From & To & Energy & Price \\
6 & & L8 & 42 & 8 & 1 & G1 & Local Grid & 20 & 9
\end{tabular}

However, the power system constraints limited the supplied energy to this MG to 197

KWh because of the Line 17-22 loading limit. The MG1 agent in the inter-MG layer should create a new block of transactions for MG1 BC based on detailed analysis form participants in MG1. For this purpose, loads with higher energy bids are approved first. It can be seen that L7 was eager to pay up to 14 cents/kWh for $65 \mathrm{KWh}$. Therefore, the agents assign more expensive providers to this unit. Afterward, other loads are evaluated based on their demand and bids, as reported in Table 6.5. Finally, the MG1 must decrease the total load by $200 \mathrm{KWh}$ for the upcoming market interval to avoid any energy interruption in the system. Thus, consumers who are not the winner in the market should be disconnected from the MG1 internal network. 


\subsection{Summary}

In this chapter, we introduced a hierarchical Blockchain-based (HBC) energy transaction framework for energy transactions in a multi-microgrid system. It includes two layers of blockchains; the first is developed for transactions within each MG using conventional $\mathrm{BC}$ technology, the second one is used for inter-MG transactions where we developed a ledger summarization method and introduced an equivalent model for microgrids to participate in inter-MG transactions. We developed the proposed framework using Java programming language and verified it by deploying the algorithm in a threeMicrogrid system. Results demonstrated that the HBC algorithm can successfully addresses the feasibility assessment of inter-MG energy transactions in a multi-microgrid and improve the privacy of participants in the inter-MG market. More detail and data are in Appendix II. 


\section{Chapter 7 Optimal Operation of Unbalanced Hybrid AC-DC Microgrids}

As mentioned in Chapter 6, the unbalanced loading in distribution networks significantly affects the optimal operation of the system, notably by limiting the transmission lines' loading. In this chapter, we propose an OPR scheme between and within ICs in unbalanced hybrid AC-DC microgrids. This OPR scheme minimizes the PIF at the PCC, active power losses, and voltage deviation indices for microgrids in grid-connected operating mode. These goals are achieved through a multi-objective optimization model by an optimal distribution of mobile loads between available charging stations and, at the same time, optimal power routing within three phases of three-phase four-leg AC/DC converters. Numerical results obtained from implementing the developed method on the modified IEEE 13-bus system, as an unbalanced hybrid microgrid, and IEE 34-bus test system, as an unbalanced distribution system, demonstrate that the developed OPR algorithm is successful in satisfying the optimization goals. For this purpose, four case studies are defined and studied to demonstrate the unique features of the developed OPR comparing with other power routing schemes. In addition to simulation results, the OPR scheme between ICs is realistically implemented at the FIU smart grid testbed to show the effect of the power routing on energy losses reduction.

\subsection{Nomenclature}

The multi-objective optimization problem contains many variables and indices. Therefore, in this section, a nomenclature is presented in Table 7.1 to facilitate following the manuscript. Other symbols are defined in the text as they appear. 
Table 7.1: Nomenclature for Chapter 7.

\begin{tabular}{|c|c|}
\hline A & Coefficient matrix. \\
\hline$A P L I_{c}$ & Active Power Losses Index for $\mathrm{c}^{\text {th }}$ case study. \\
\hline$A P L I^{\max }$ & Maximum APLI among all case studies. \\
\hline$\tilde{A}_{c}$ & Normalized APLI ${ }_{C}$ \\
\hline$A v e\left|V_{i}^{k}\right|$ & Average voltage magnitude of three phases at $\mathrm{i}^{\text {th }}$ bus. \\
\hline$B$ & The vector of upper bands. \\
\hline$D$ & The number of the buses connected to ICs. \\
\hline$f_{i}(X)$ & $\mathrm{i}^{\mathrm{th}}$ objective function. \\
\hline$I_{j}^{k}$ & Current of $\mathrm{k}^{\text {th }}$ phase of $\mathrm{j}^{\text {th }}$ line. \\
\hline$I_{j}^{\max }$ & Maximum Current for each phase of $\mathrm{j}^{\text {th }}$ line. \\
\hline$k, m$ & Phase indicators in a 3-phase system $(a, b, c)$. \\
\hline$L$ & The number of transmission lines. \\
\hline$M O I_{c}$ & Microgrid Operating Index for $\mathrm{c}^{\text {th }}$ case study. \\
\hline$M O I^{\max }$ & Maximum MOI among all case studies. \\
\hline$\tilde{M}_{c}$ & Normalized $\mathrm{MOI}_{\mathrm{C}}$ \\
\hline$N$ & Number of buses in the system. \\
\hline$P I F I_{C}$ & Power Imbalance Factor Index for $\mathrm{c}^{\text {th }}$ case study. \\
\hline$P I F I^{\max }$ & Maximum PIFI among all case studies. \\
\hline$\tilde{P}_{c}$ & Normalized PIFI ${ }_{C}$. \\
\hline$P_{d}^{k}, Q_{d}^{k}$ & Active and reactive power set point for $\mathrm{k}^{\text {th }}$ phase of $\mathrm{d}^{\text {th }} \mathrm{ICs}$. \\
\hline$P_{d}^{r e f, k}, Q_{d}^{r e f, k}$ & Reference active and reactive power for $\mathrm{k}^{\text {th }}$ phase of $\mathrm{d}^{\text {th }} \mathrm{ICs}$. \\
\hline$P_{d}^{O p t, k}, Q_{d}^{O p t, k}$ & Optimum active and reactive power set points for $\mathrm{k}^{\text {th }}$ phase of $\mathrm{d}^{\text {th }} \mathrm{ICs}$. \\
\hline$P_{d}^{\min } \max ^{\max }$ & Minimum/Maximum active power limit for each phase of $\mathrm{d}^{\text {th }} \mathrm{ICs}$. \\
\hline$P T_{d}$ & Total active power of $\mathrm{d}^{\text {th }}$ ICs. \\
\hline$P T_{d}^{\min }{ }_{d}^{\max }$ & Minimum/Maximum total active power limit for $\mathrm{d}^{\text {th }} \mathrm{ICs}$. \\
\hline$P_{i}^{k}+j Q_{i}^{k}$ & Injected complex power from the $\mathrm{k}^{\text {th }}$ phase of the $\mathrm{i}^{\text {th }}$ bus to the grid. \\
\hline$Q_{d}^{\min } \max ^{\max }$ & Minimum/Maximum reactive power limit for each phase of $\mathrm{d}^{\text {th }} \mathrm{ICs}$. \\
\hline$Q T_{d}^{\min } \max ^{\max }$ & Minimum/Maximum total reactive power limit for $\mathrm{d}^{\text {th }} \mathrm{ICs}$. \\
\hline$r$ & The number of objective functions. \\
\hline$R_{j}^{k}$ & The resistance of the $j^{\text {th }}$ line. \\
\hline$S^{k}$ & The complex power of $\mathrm{k}^{\text {th }}$ phase at the PCC. \\
\hline$\hat{S}$ & The average complex power at the PCC. \\
\hline $\bar{S}$ & The average of the absolute power at the PCC. \\
\hline$V_{i}^{k}$ & The voltage of $\mathrm{k}^{\text {th }}$ phase of $\mathrm{i}^{\text {th }}$ bus. \\
\hline$V_{i}^{\min _{i}^{\max }}$ & Minimum/Maximum voltage magnitude at $\mathrm{i}^{\text {th }}$ bus. \\
\hline$V_{\text {ref }}$ & Reference voltage. \\
\hline$V I F_{i}$ & Voltage Imbalance Factor at $i^{\text {th }}$ bus. \\
\hline$V D I_{c}$ & Voltage Deviation Index for $\mathrm{c}^{\text {th }}$ case study. \\
\hline$V D I^{\max }$ & Maximum VDI among all case studies. \\
\hline$\tilde{V}_{c}$ & Normalized VDI $\mathrm{C}$. \\
\hline$w_{i}$ & Weighting factors for the $i^{\text {th }}$ objective optimization. \\
\hline$X$ & Set of decision variables. \\
\hline $\begin{array}{l}Y_{i h}^{k m} \\
Y^{a b c}\end{array}$ & Element of $\mathrm{Y}$ matrix between $\mathrm{k}^{\text {th }}$ phase of $\mathrm{i}^{\text {th }}$ bus, and $\mathrm{m}^{\text {th }}$ phase of $\mathrm{h}^{\text {th }}$ bus. \\
\hline & The admittance matrix between $1^{\mathrm{ci}}$ and $\mathrm{h}^{\mathrm{m}}$ buses. \\
\hline
\end{tabular}



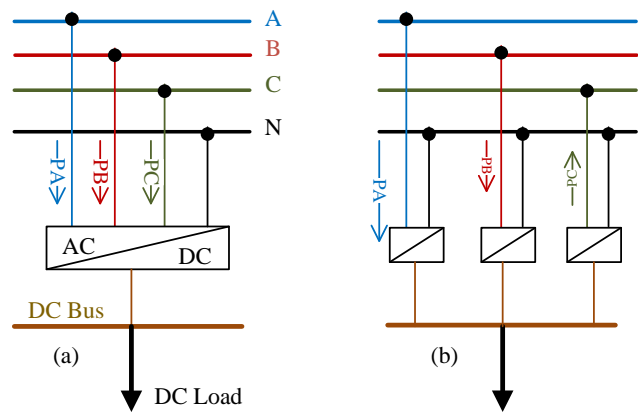

Figure 7.1: Symmetric (a) and asymmetric (b) control models for Interlinking converters.

\subsection{Control models of Interlinking Converters}

Usually, a three-phase interlinking converter is operated as an asymmetric converter where all three-phase loadings are the same, and the corresponding switching pattern is deployed for three phases considering phase shifting between three phases. However, it is possible to operate a converter unsymmetrically. It means that different phases could have different loading indices due to the system requirements. For the asymmetric operation of three-phase converters, we should replace them with four-lag converters in four-wire AC systems, or we can use three-phase three-lag converters when $\Delta / Y$ transformers are implemented [110]. Therefore, we can model a three-phase converter as three single-phase converters, as shown in Figure 7.1. In both symmetric and asymmetric control models, we can use different techniques for active and reactive power of the converter, including PQ control, droop control, and V/F control method. Since we are studying a grid-connected microgrid, we consider the PQ control strategy for our ICs [183].

Using this control method, the switching activates within the converter is controlled in such a way that the converter output satisfies active and reactive power according to its assigned PQ references as shown by (7-1) and (7-2).

$$
P_{d}^{k}=P_{d}^{r e f, k}
$$




$$
Q_{d}^{k}=Q_{d}^{r e f, k}
$$

Therefore, optimal tuning of PQ references will result in the optimal operation of hybrid AC-DC microgrids, as we will describe in the next sections. It should be mentioned that other control techniques like droop or V/F control methods are more appropriate for islanding operation mode of Microgrids.

\subsection{The developed OPR scheme}

The flowchart of the developed OPR method is shown in Figure 7.2 for $\mathrm{i}^{\text {th }}$ operation interval. As it can be seen in this figure, the flowchart contains an optimization algorithm which uses the system model and unbalanced power flow calculations during the optimization process to optimize the operation of the system by routing the power between and within ICs.

The flowchart starts with updating the system model for the $i^{\text {th }}$ operating interval. It means that all forecasted renewable generations, load estimation, and EVs charging requests are collected to modify the microgrid model for the upcoming operating interval. Afterward, a modified PSO algorithm tries to solve a multi-objective optimization model aiming at minimizing the power imbalance factor at the PCC, active power losses, and voltage deviation index. The output of this optimization is optimum PQ references for each phase of ICs in the system.

The ICs in this system is used by CSs to charge EVs. Therefore, to meet the optimization output, an effective EV re-routing mechanism is required. As it can be seen in the flowchart, after PSO convergence, the output is sent to an agent for real-time management of en-route EVs. 


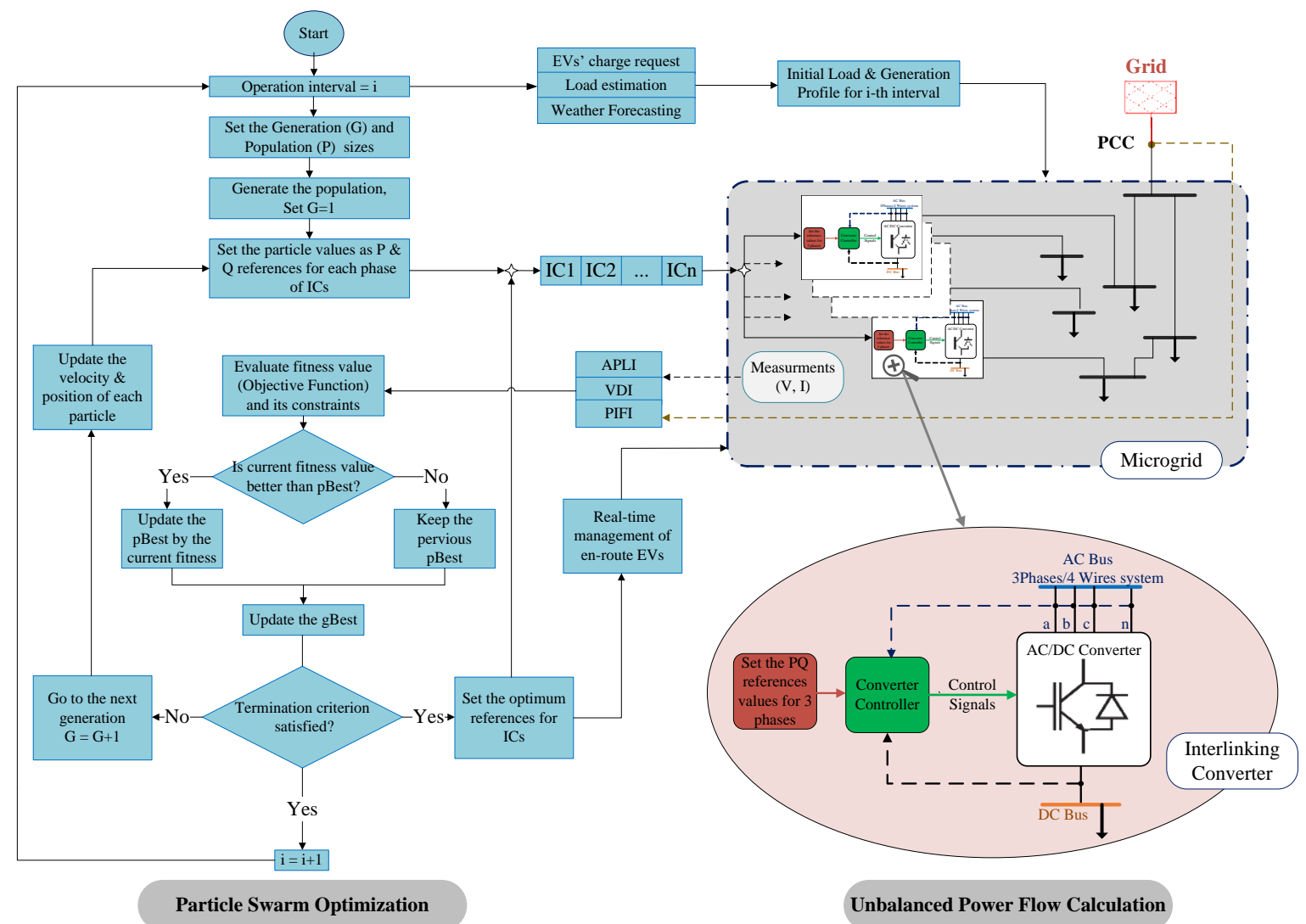

Figure 7.2: The proposed optimal power routing scheme for unbalanced hybrid AC-DC MGs in gridconnected mode using PSO algorithm and unbalanced power flow calculations.

In [184], the authors have proposed a cognitive price-based strategy for real-time management of en-route EVs, which can motivate the EVs' owners to go to determined CSs based on the system requirement. Since this topic is out of the scope of this dissertation, here in this work, we assume that optimal load sharing between ICs is doable.

\subsubsection{Multi-objective optimization model}

As mentioned before, in this problem, there are three objective functions to be optimized simultaneously. Therefore, a multi-objective optimization model is needed. A multi-objective optimization model is used for an optimization problem with several objective functions that may contradict each other. For this kind of optimization problem, the concept of Pareto optimality can help to find an optimum solution. If this solution 
exists, it provides an efficient solution, which means that it cannot be improved for one of the objective functions without having a negative effect on other objective functions [185]. Generally, a multi-objective optimization model can be written as follow:

$$
\min F(X)=\left(f_{1}(X), f_{2}(X), \ldots, f_{r}(X)\right)
$$

Subject to: $A X \leq B$

There are several solution methods for this problem, such as the weighted-sum method, game-theoretical techniques, etc. Between all these methods, the most common method is the weighted-sum method [186], where it uses weighting factors to combine all objective functions as a single objective function shown by (7-4).

$$
\min F(X)=w_{1} f_{1}(X)+w_{2} f_{2}(X)+\ldots+w_{r} f_{r}(X)
$$

Subject to: $A X \leq B$

Choosing appropriate weighting factors for objective functions is one of the most important issues in this method. These factors are selected based on the importance of each objective function, and usually, the trial and error method is deployed in this regard. In this dissertation, three objective functions are defined for the optimization problem, and the weighted-sum method is used to solve the problem. The objective functions are defined as follow:

1. $f_{1}(X)=$ APLI

This index is defined as total active power losses by transmission lines within the microgrid and is calculated by (7-5).

$$
A P L I=\sum_{j=1}^{L} \sum_{k} R_{j}^{k}\left|I_{j}^{k}\right|^{2}
$$




\section{2. $f_{2}(X)=$ VDI}

The voltage deviation index is defined by (7-6). where, the voltage deviation index at $\mathrm{i}^{\text {th }}$ bus of the system is defined by (7-7) as the maximum value of voltage deviation from the reference voltage magnitude (e.g. 1 pu), between three phases.

$$
\begin{gathered}
V D I=\sum_{i=1}^{N}\left(V D I_{i}\right)^{2} \\
V D I_{i}=\left(\left|V_{i}^{k}\right|-\left|V_{\text {ref }}\right|\right)^{\max }
\end{gathered}
$$

3. $f_{3}(X)=$ Power imbalance factor index (PIFI)

To keep the power balance at the PCC, the power imbalance factor should be minimized at this point, therefore, the PIFI is defined by (7-8) which shows the maximum deviation of complex power between three phases from their average value.

$$
P I F I=\left(\frac{1}{\bar{S}}\right) \times \max \left|S^{k}-\hat{S}\right|
$$

Where,

$$
\begin{gathered}
\hat{S}=\left(\frac{1}{3}\right) \cdot \sum_{k} S^{k} \\
\bar{S}=\left(\frac{1}{3}\right) \cdot \sum_{k}\left|S^{k}\right|
\end{gathered}
$$

Hence, using the weighted-sum method and defining the problem constraints, the OF for this problem along with all constraints are presented by (7-11)-(7-19).

$$
\min O F=w_{1} \times A P L I+w_{2} \times V D I+w_{3} \times P I F I
$$

Subject to: 


$$
\begin{gathered}
P_{i}^{k}+j Q_{i}^{k}=V_{i}^{k} \sum_{h=1}^{N} \sum_{m}\left(Y_{i h}^{k m}\right)^{*}\left(V_{h}^{m}\right)^{*} \\
\sum_{d=1}^{D}\left(\sum_{k} P_{d}^{k}\right)=\sum_{d=1}^{D} P T_{d} \\
P T_{d}^{\text {min }} \leq\left(\sum_{k} P_{d}^{k}\right) \leq P T_{d}^{\max } \\
Q T_{d}^{\text {min }} \leq\left(\sum_{k} Q_{d}^{k}\right) \leq Q T_{d}^{\max } \\
P_{d}^{\min } \leq P_{d}^{k} \leq P_{d}^{\max } \\
Q_{d}^{\min } \leq Q_{d}^{k} \leq Q_{d}^{\max } \\
V_{i}^{\min } \leq\left|V_{i}^{k}\right| \leq V_{i}^{\max } \\
I_{j}^{k} \leq I_{j}^{\max }
\end{gathered}
$$

Equation (7-12) shows the unbalanced power flow equations as described in [187], where the admittance matrix of the microgrid is obtained by (7-20).

$$
Y_{i h}^{a b c}=\left[\begin{array}{lll}
Y_{i h}^{a a} & Y_{i h}^{a b} & Y_{i h}^{a c} \\
Y_{i h}^{b a} & Y_{i h}^{b b} & Y_{i h}^{b c} \\
Y_{i h}^{c a} & Y_{i h}^{c b} & Y_{i h}^{c c}
\end{array}\right]
$$

The load balance constraint is presented by (7-13) to make sure that the total load before and after the power routing is the same while the load could be transferred between ICs and also within three phases of each IC. The maximum and minimum capacity limits for active and reactive power of ICs are determined by (7-14) and (7-15), respectively. In addition, the active and reactive power limits for each phase of ICs, are reported by (7-16) and (7-17). Finally, the voltage magnitudes of each bus and feeders' currents should not exceed their pre-defined limits, as shown by (7-18) and (7-19). By calculating the final active and reactive power values for each phase of the three-phase ICs, the PQ references are updated and set as below. 


$$
\begin{aligned}
& P_{d}^{r e f, k}=P_{d}^{o p t, k} \\
& Q_{d}^{r e f, k}=Q_{d}^{o p t, k}
\end{aligned}
$$

Assuming that the re-routing mechanism is successful in managing mobile loads, these reference values are sent to ICs' controllers for tuning their active and reactive power outputs.

\subsubsection{Particle Swarm Optimization (PSO)}

Generally, PSO is a proper optimization algorithm for power system studies, especially when nonlinear power flow equations are included in the optimization problem [188]. The PSO can also be implemented for solving multi-objective optimization problems in power systems. However, other optimization algorithms such as MOGA, NSGA, Taube search algorithm, etc., could also be deployed for this purpose. In this dissertation, because of using the weighted-sum method, the multi-objective optimization model is converted to a single-objective optimization problem. Therefore, the modified PSO algorithm can be considered as a right choice for this optimization problem because it is strong enough to converge to an optimal solution over an acceptable solution time.

The modified PSO algorithm in this research is equipped with band coefficients and variables' direction control mechanisms, which accelerate the PSO to move toward the optimal point while it does not lose the first feasible solution for the problem. The detailed description of this modification was presented in [189]. Figure 7.2 demonstrates the steps in the PSO algorithm in detail. As can be seen in this flowchart, the optimization process is started by generating the first population of the PSO and selecting variables' values. Afterward, these values are set in the network model, and an unbalanced power flow is 
executed to obtain the results which are used to evaluate the objective function. In each iteration, the global best is updated based on the best solution of that generation. Considering the best existing solution, variables' speeds are determined, and the new generation of particles is created, and this process continues until one of the optimization criteria are reached. The output of PSO contains the optimum PQ references for each phase of ICs. The challenging issue for PSO algorithm is to find appropriate generation and population sizes, which are different for each application. In this study, we determine these values for each system using the trial and error method. By finding the optimum solution of OPR, the load management between ICs is doable by the real-time rerouting mechanism.

\subsubsection{Microgrid operating index}

Since the developed OPR scheme includes a multi-objective optimization which tries to minimize several indices, and also to compare the results of this method to the base study case and other case studies with algorithms that focus on some of the indices, we can define the MOI and its normalized value as an indicator that reflects the microgrid operating features as shown by (7-23) and (7-24).

$$
\begin{gathered}
\widetilde{M}_{c}=\frac{M O I_{c}}{M O I^{\max }} \\
M O I_{c}=\left(\tilde{A}_{c}+\tilde{V}_{c}+\tilde{P}_{c}\right)
\end{gathered}
$$

where in these equations:

$$
\begin{gathered}
\tilde{A}_{c}=\frac{A P L I_{c}}{A P L I^{\max }} \\
\tilde{V}_{c}=\frac{V D I_{c}}{V D I^{\max }}
\end{gathered}
$$

Using this index, it is possible to compare different case studies with different objective functions. 
Table 7.2: Power routing mechanism and objective functions for case studies.

\begin{tabular}{|l|c|c|c|c|c|}
\hline \multirow{2}{*}{ Study Case } & \multicolumn{2}{|c|}{ Power Routing Mechanism } & \multicolumn{3}{c|}{$\begin{array}{c}\text { Objectives } \\
\text { Minimizing) }\end{array}$} \\
\cline { 2 - 6 } & Within ICs & Between ICs & PIFI & APLI & VDI \\
\hline C1- Base Case & $\boldsymbol{x}$ & $\boldsymbol{x}$ & $\mathbf{x}$ & $\mathbf{x}$ & $\mathbf{x}$ \\
\hline C2- Ref [118] & $\checkmark$ & $\boldsymbol{x}$ & $\checkmark$ & $\mathbf{x}$ & $\mathbf{x}$ \\
\hline C3- Ref [119] & $\checkmark$ & $\boldsymbol{x}$ & $\checkmark$ & $\checkmark$ & $\mathbf{x}$ \\
\hline C4- Developed OPR & $\checkmark$ & $\checkmark$ & $\checkmark$ & $\checkmark$ & $\checkmark$ \\
\hline
\end{tabular}

From this definition, it is obviously clear that lower $\widetilde{M}_{c}$ for a case study shows the better performance of the microgrid regarding the active power losses, voltage deviations and power imbalance factor at the PCC.

\subsection{Numerical results}

To compare the results of the developed method by other methods described in [118] and[119], four case studies are defined in Table 7.2. As can be seen in this table, C1 to C4 stands for case studies 1 to 4 , respectively. C1 is the base case without any control and optimization algorithm. In case two (C2), only the power routing mechanism is deployed to remove the unbalances at the PCC using a supervisory control scheme as described in [118]. This method tries to devote the unbalancing value at the PCC to the ICs regarding their capacities C3 is reflecting the results from the proposed method in [119], which minimizes the active power losses and PIF at the PCC hierarchically. And finally, C4, which is the developed OPR method in this dissertation that uses the power routing between and within ICs aiming at minimizing the active power losses, PIF at the PCC, and the voltage deviations of all buses in the microgrid from the nominal value.

All these case studies will be analyzed through numerical results by implementing these methods on the modified IEEE 13 bus system as a highly unbalanced hybrid AC-DC microgrid. Furthermore, to prove the capabilities of the developed OPR scheme in distribution systems, this method was also examined on the IEEE 34-bus system as an 


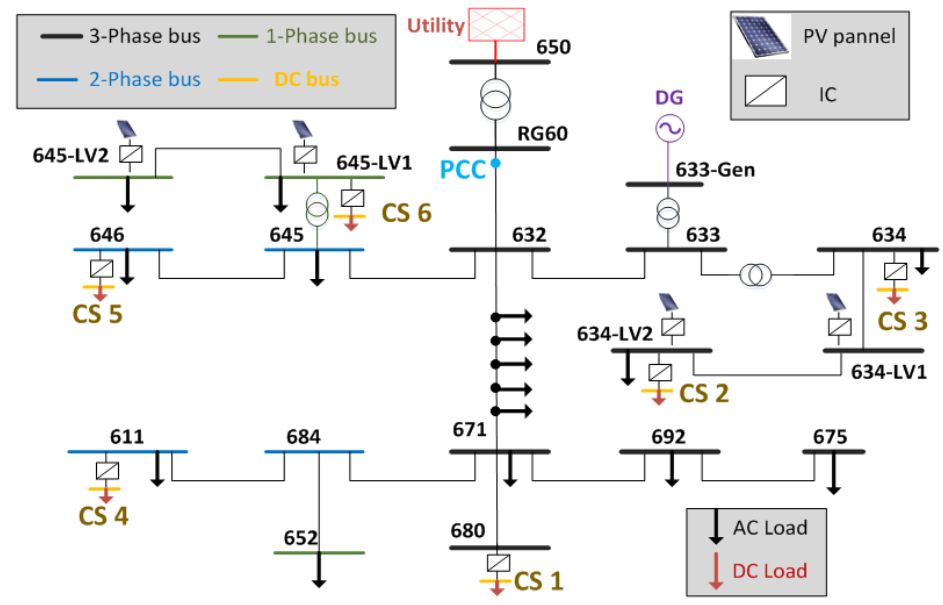

Figure 7.3: The modified IEEE 13-bus system as an unbalance hybrid AC-DC microgrid.

unbalanced distribution network. Finally, the FIU smart grid testbed is studied to show the effect of OPR between ICs in losses reduction in an actual smart microgrid.

\subsubsection{Modified IEEE 13 bus system}

The modified IEEE 13-bus system is shown in Figure 7.3. In [190], the details of the modifications have been reported. In this microgrid, CS1, CS2, and CS3 are charging stations with three-phase interlinking converters (IC\#1, IC\#2, and IC\#3) while CS4, CS5, and CS6 indicate charging stations containing single-phase interlinking converters.

In this dissertation, the system is modeled in DigSilent PowerFactory2019, as a professional power system software and the DPL is used to develop all algorithms related to four case studies. Figure 7.4 and 7.5 demonstrate the active power losses and power imbalance factor of the system during a 24-hour operation interval for $\mathrm{C} 1$ to $\mathrm{C} 4$. As can be seen in Figure 7.4, maximum and minimum active power losses are reported for $\mathrm{C} 1$ and $\mathrm{C} 4$, respectively. Figure 7.5 shows that in $\mathrm{C} 2, \mathrm{C} 3$, and $\mathrm{C} 4$, the $\mathrm{PIF}$ at the $\mathrm{PCC}$ is zero while in $\mathrm{C} 1$, the PIF is fluctuating between 23 to $27 \%$. It is because of this fact that the objective functions in $\mathrm{C} 2, \mathrm{C} 3$, and $\mathrm{C} 4$ are aimed to minimize the PIF at the PCC. 


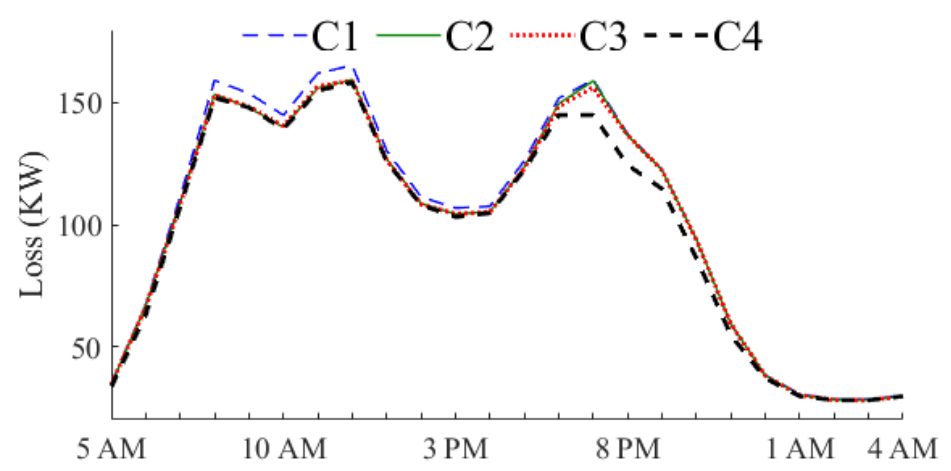

Figure 7.4: Energy Losses for C1 to C4

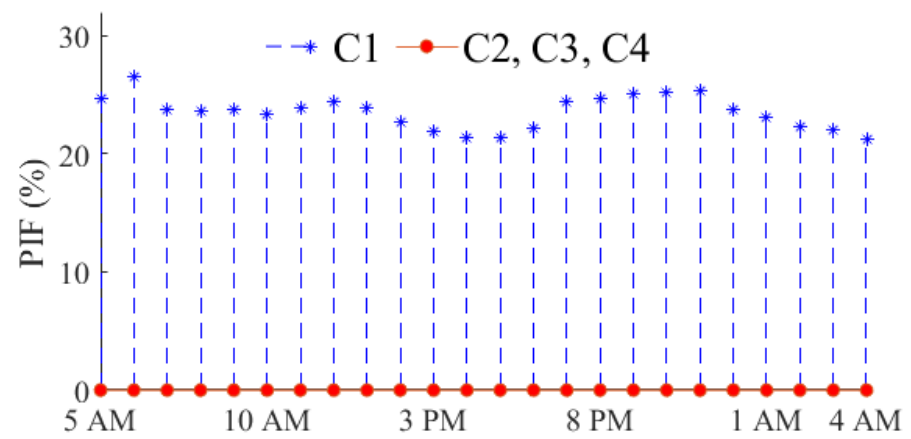

Figure 7.5: $\mathrm{PIF}$ at the $\mathrm{PCC}$ for $\mathrm{C} 1$ to $\mathrm{C} 4$

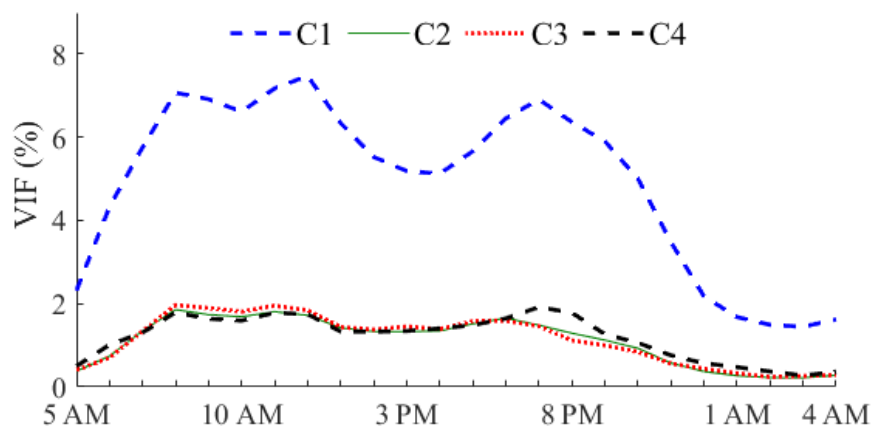

Figure 7.6: VIF at bus 671 for $\mathrm{C} 1$ to $\mathrm{C} 4$

To compare the voltage profiles in all case studies, in addition to VDI, which is used in the optimization model, we can calculate the VIF by (7-28) for each bus of the system to measure the unbalances between three phases in unbalanced microgrids.

$$
V I F_{i}=\frac{\left(\left|V_{i}^{k}\right|-A v e\left|V_{i}^{k}\right|\right)^{\max }}{A v e\left|V_{i}^{k}\right|}
$$




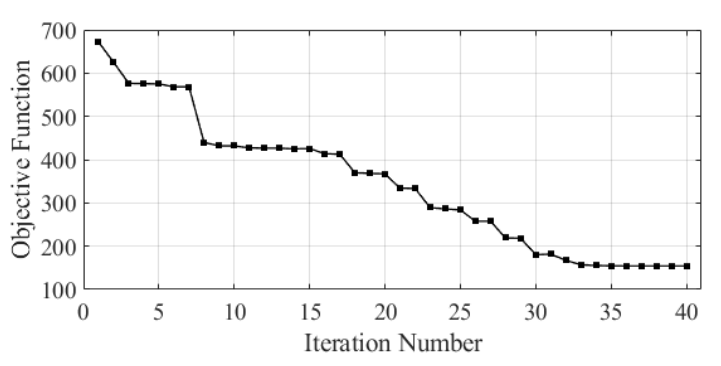

Figure 7.7: Objective function values for each iteration of PSO

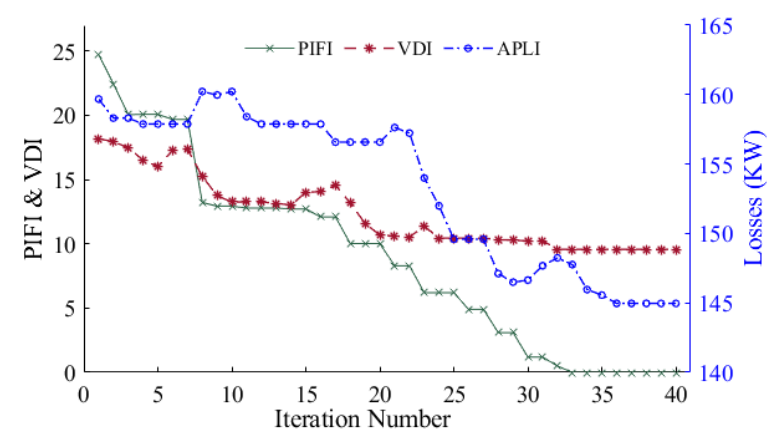

Figure 7.8: The values of PIF, VDI and APLI for each iteration of PSO

Figure 7.6 shows the voltage imbalance factors at bus 671 of the system for all case studies. It is observed that the VIF is fluctuating between 2 to $8 \%$ in $\mathrm{C} 1$ while it is less than $2 \%$ in $\mathrm{C} 2, \mathrm{C} 3$, and $\mathrm{C} 4$ over this 24 -hour operating interval. It means that in all the methods in $\mathrm{C} 2, \mathrm{C} 3$, and $\mathrm{C} 4$, the voltage profile of the system buses is improved. This fact will be reported in detail when system indices are calculated.

To validate the results from each case study, here, we focus on the results for one operating interval. Finally, we will calculate the system indices to compare the results from four case studies. The numerical results are reported for 8 PM in detail by Fig 7.7 to Figure 7.13. Since the main goal in this study is to keep the power balance at the PCC and to achieve this goal in $\mathrm{C} 4$ (the developed OPR optimization model), we need to consider a high weighting factor for PIFI as shown by (7-29).

$$
w_{3}>w_{2}, w_{1}
$$

In this study, and as can be seen in Figure 7.8, the initial values for PIFI, VDI, and APLI are around 25, 18, and 160, respectively. In this case, and to dominate the impact of PIFI on the final objective function value, at first, we set (the weighting factor for PIFI) while we adjusted. Afterward, and by running the optimization, we observed that the PSO minimizes the PIFI, and its effect on the other indexes is negligible. 


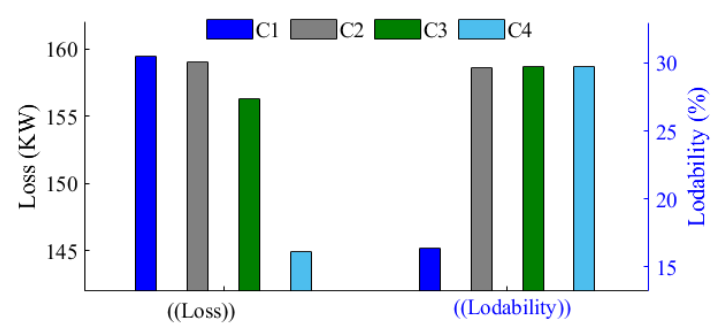

Figure 7.9: Microgrid active power losses and loadability for $\mathrm{C} 1$ to $\mathrm{C} 4$

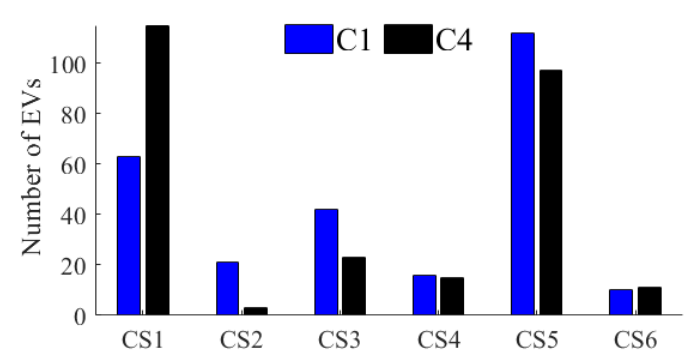

Figure 7.10: EVs distribution in base and optimum cases

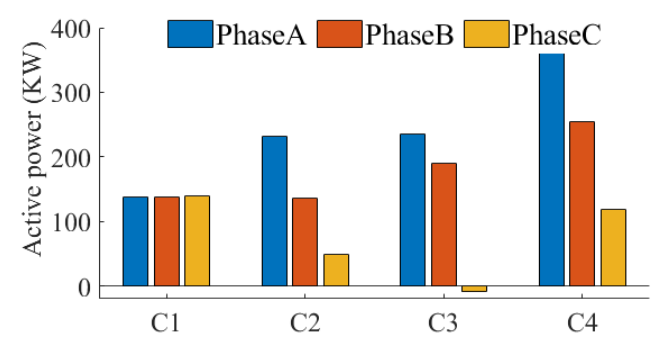

Figure 7.11: Active power value for each phase of IC\#1 for $\mathrm{C} 1$ to $\mathrm{C} 4$

Thus, we gradually decreased from 30 step by step and ran the optimization receptively.

Finally, our simulation results demonstrated that if the, by choosing, the final objective function could satisfy our expectations for minimizing the PFIF as well as reducing the VDI and active power losses.

In this study, a Core i7 CPU $3.40 \mathrm{GHz}$ with $8 \mathrm{~GB}$ of memory (RAM) computer is used for system simulation and running the optimization program. For the modified 13-bus system, we define the population size and maximum generation size of the PSO by 150 and 40, respectively. We also determined these values using the trial and error method. But the difference is that at first, we selected very high values for both the population size and maximum generation size by 400 and 250, respectively, and run the PSO several times. The reason was to find an optimum solution without considering the solution time (in this case, the computational time was around 197 seconds). 


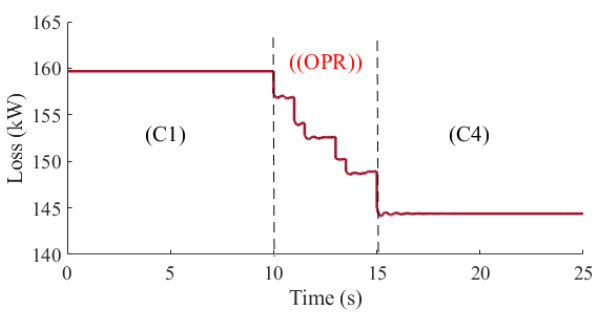

Figure 7.12: Dynamic simulation of Microgrid losses for $\mathrm{C} 1 \& \mathrm{C} 4$

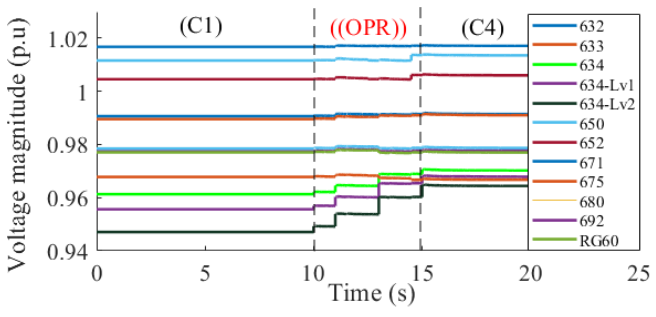

Figure 7.13: Dynamic simulation of Voltage profiles for $\mathrm{C} 1 \& \mathrm{C} 4$

Since decreasing the population size and maximum generation size significantly reduce the computational time, we set a $3 \%$ error for a final solution while we could lower the solution time form 197 seconds (for the population size and maximum generation size of 400 and 250, respectively) to 38.6 seconds for population size and maximum generation size of the PSO by 150 and 40, respectively. Numerical results show the algorithm is fast enough for this application, which tries to optimize the system for the next operating interval. Figure 7.7 shows the value of the objective function during the optimization, and Figure 7.8 demonstrates the values of PIFI, VDI, and APLI in this optimization process. As can be observed from these two figures, minimizing the PIFI is dominant to VDI and APLI, and it finally goes to zero at the 33rd iteration of the PSO. The general trend of APLI and VDI shows that they are also decreasing, but they have some fluctuations before reaching their final optimum values. Figure 7.9 shows the microgrid active power losses and loadability for all case studies.

As can be seen in this figure, the developed OPR scheme (C4) will result in the least active power losses and the same loadability index comparing with $\mathrm{C} 2$ and $\mathrm{C} 3$. The distribution of mobile loads for the first three case studies would be the same as shown by Figure 7.10 because, in these cases, we do not shift the loads between ICs while in C4, the developed OPR model optimizes the distribution of EVs between ICs. 
Table 7.3: System indices at 8 PM.

\begin{tabular}{c|lccc} 
STUDY CASE & $\boldsymbol{P I F I}_{\boldsymbol{c}}$ & $\boldsymbol{V} \boldsymbol{D} \boldsymbol{I}_{\boldsymbol{c}}$ & $\boldsymbol{A P \boldsymbol { L } \boldsymbol { I } _ { \boldsymbol { c } }}$ & $\widetilde{\boldsymbol{M}}_{\boldsymbol{c}}$ \\
\hline (C1) & 24.43 & 0.0673 & 159.4452 & 1.0000 \\
(C2) & 0.00 & 0.0261 & 159.0393 & 0.4618 \\
(C3) & 0.00 & 0.0252 & 156.2815 & 0.4515 \\
(C4) & 0.00 & 0.0192 & 144.9713 & 0.3982
\end{tabular}

It can be seen that the number of EVs for CS1 is increased significantly while it is decreased for CS2, CS3, and SC5. CS4 and CS6 do not experience vast differences. Figure 7.11 represents the active power for each phase of IC\#1 as an example of three-phase ICs in the system. It is clear that except in $\mathrm{C} 1$, in other case studies, this IC is operated asymmetrically. It is because of this fact that the asymmetric operation of this converter results in minimum PIFI at the PCC (in this case, PIFI is zero) and also improvement in the system indices.

In addition to static simulations for this 24-hour operating interval, quasi-dynamic simulations have been executed for this operation interval to show the dynamic performance of the system regarding the proposed OPR method in C4. Figure 7.12 illustrates the active power losses, and Figure 7.13 shows the voltage profile of all AC buses in the system for the base case (C1) and the optimum case (C4). We simulated the power routing schemes gradually to see the changes. As can be seen in this figure, and also Figure 7.9, the system loss is decreased from $159 \mathrm{~kW}$ in $\mathrm{C} 1$ to $144 \mathrm{~kW}$ in C4. From Figure 7.13 , it can be concluded that the optimum power routine scheme has improved the voltage profile significantly. For example, the voltage of bus 632-LV2 in $\mathrm{C} 1$ is less than 0.95 p.u. while it is in an acceptable range in $\mathrm{C} 4$. To evaluate the best performance of the system between these four case studies, we need to calculate the normalized value of the microgrid operating index $\left(\widetilde{M_{c}}\right)$ for all case studies, as shown in Table 7.3. Since the lowest value reflects the better performance of the system, following we can classify all case studies. 


$$
\widetilde{M}_{C 4}<\widetilde{M}_{C 3}<\widetilde{M}_{C 2}<\widetilde{M}_{C 1}
$$

This classification shows that $\mathrm{C} 4$, the developed OPR method, has the minimum microgrid operating. The second rank of this classification is for $\mathrm{C} 3$, the hierarchical power routing scheme. $\mathrm{C} 2$, the supervisory control method, has the third rank and $\mathrm{C} 1$, system without any control and optimization mechanism, has the biggest system operating index among these case studies. Although both supervisory control scheme and hierarchical power routing schemes are successful in improving the operation of unbalanced hybrid microgrids, the developed multi-objective optimization method leads to best results for optimal operation of hybrid microgrids.

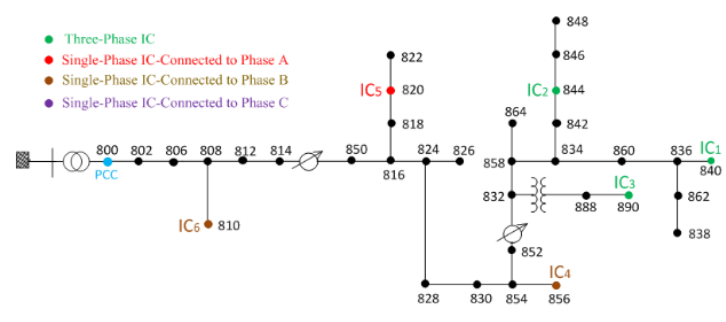

Figure 7.14: Modified IEEE 34-bus test system with six ICs

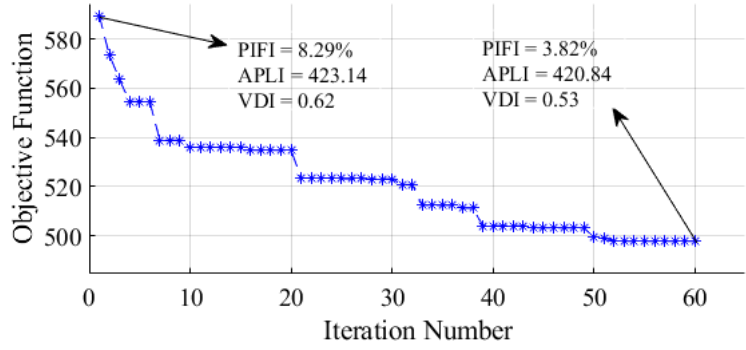

Figure 7.15: Objective function values for IEEE 34bus system

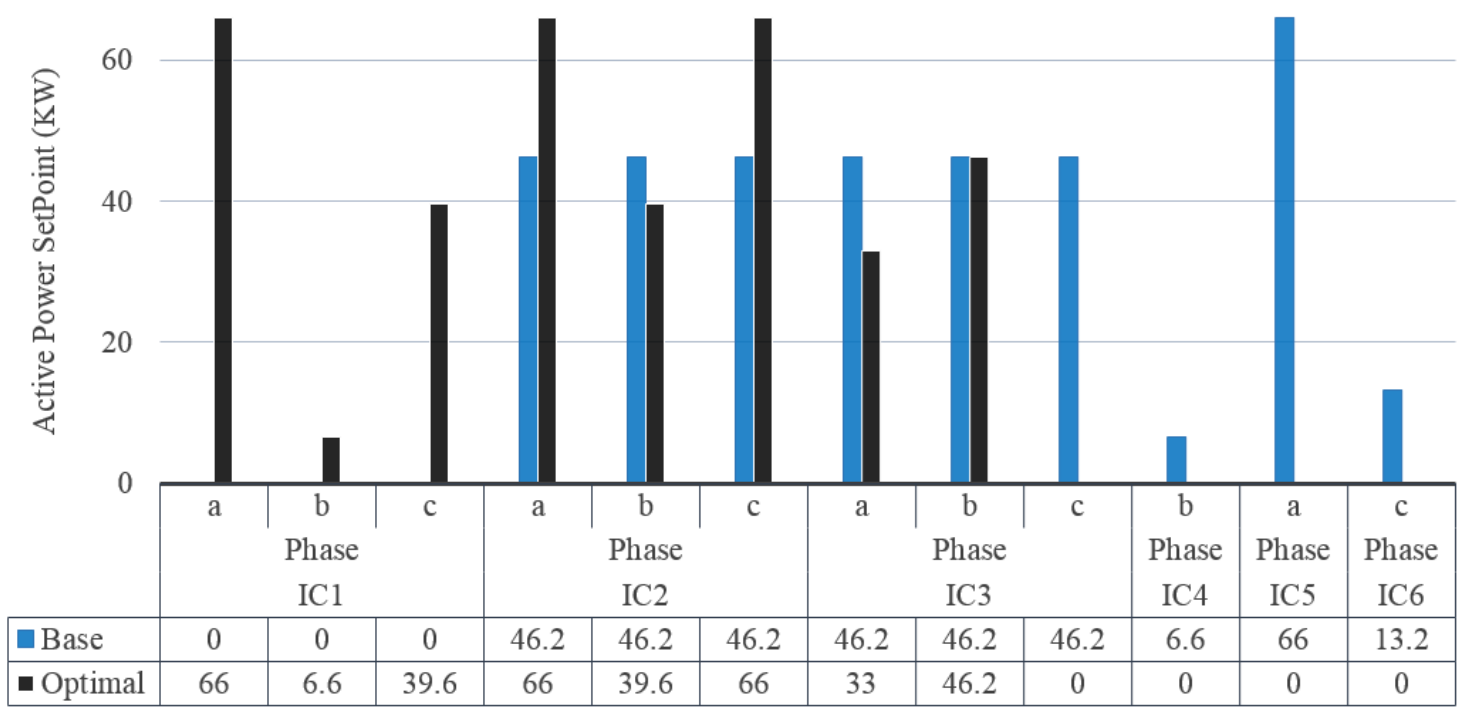

Figure 7.16: Reference active power for ICs for base and optimal Scenarios 


\subsubsection{IEEE 34-bus test system}

The developed OPR method in this dissertation can also be implemented in unbalanced distribution systems containing three-phase four-leg ICs. To validate the capabilities of the developed OPR scheme in the distribution system, the IEEE 34-bus test system is selected as an example of an unbalanced distribution system [191]. In this system, we need to locate some three-phase and single-phase ICs to the system. For this purpose, we replaced three balanced loads at buses 840, 844, and 890 with three-phase, four-lag AC/DC converters. Furthermore, the single-phase loads at buses 810,820 , and 856 also replaced by singlephase AC/DC converters. The single line diagram of the system, along with all modifications, is shown in Figure 7.14. For deploying the developed OPR method, we set the population and generation sizes of the PSO as 150 and 60, respectively. Figure 7.15 shows the values of objective function from the first iteration to the last one.

The solution time for this case was 53.8 seconds, which is still in the acceptable range for this problem. As can be seen in Figure 7.15, the OPR scheme is successful in reducing PIFI from $8.29 \%$ in the first iteration to $3.82 \%$ in the last iteration. It is also decreasing the APLI and VDI from $423.14 \mathrm{KW}$ and 0.62 p.u to 420.84 and 0.53 p.u., respectively.

Figure 7.16 indicates the results of the optimization for the variables. It shows the active power set points for all phases of ICs in the system before (Base case) and after (Optimal) the optimization. The results show that the optimization is changing these values in their optimum direction to meet objective function requirements.

\subsubsection{FIU smart grid testbed}

The FIU smart grid laboratory system has been established with required hardware and software for real-time operation of a small-scale power system. This system includes four 


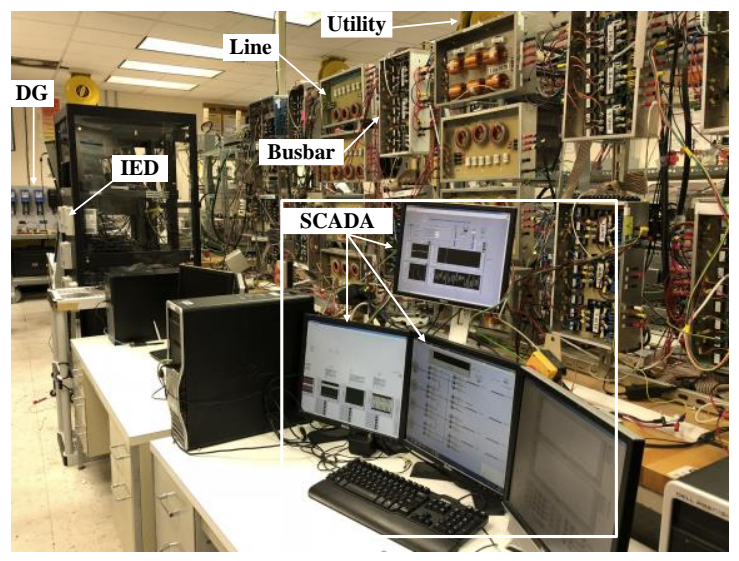

Figure 7.17: FIU smart grid testbed

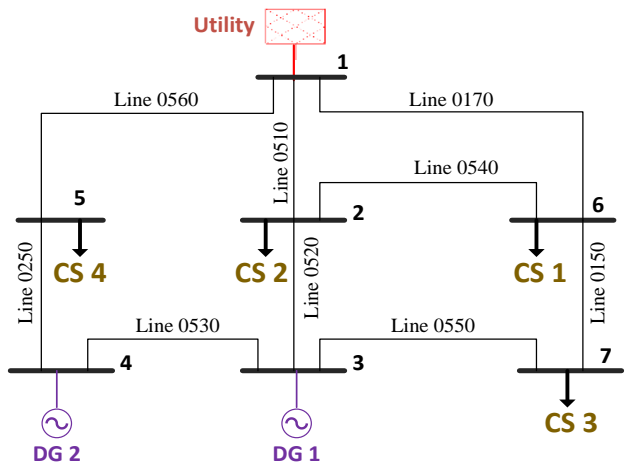

Figure 7.18: Single line diagram of the seven bus test system at FIU

\begin{tabular}{|c|c|c|c|c|}
\hline Input & Optimization & DDE & SCADA & Output \\
\hline $\begin{array}{c}\text { Initial Load \& } \\
\text { DGs' profiles }\end{array}$ & $\begin{array}{l}\text { DIGSILENT } \\
\text { DPL Script } \\
\text { (PSO) }\end{array}$ & $\begin{array}{c}\text { Dynamic Data } \\
\text { Exchange (DDE) }\end{array}$ & $\begin{array}{c}\text { LabVIEW } \\
\text { SCADA System }\end{array}$ & $\begin{array}{l}\text { Optimal load } \\
\text { profiles }\end{array}$ \\
\hline
\end{tabular}

Figure 7.19: Hardware-Software interoperability at FIU smart grid

synchronous generators along with four synchro switches, which make it possible to synchronize the generators together or with the utility. There are different types of DC and AC loads in this system. For example, there are four programmable AC loads, which can vary from 0 to $3 \mathrm{~kW}$. In this system, DAQs are implemented to monitor all nodes and branches of the microgrid. The system data are available in [192]. This system is reconfigurable and based on the case study; we can change the configuration of the system. In this system, all measurements are collected remotely via a TCP/IP connection at the SCADA system, which has been developed in LabVIEW, where it is possible to monitor and control all the system equipment, remotely. Fuses and IEDs are used in this system for protection purposes. Figure 7.17 shows the FIU smart grid testbed, and Figure 7.18 demonstrates the single-phase diagram of the 7-bus system, which contains two synchronous generators and four programmable loads. We consider these loads as charging stations, and we want to show the effect of power routing between ICs on the system losses. 


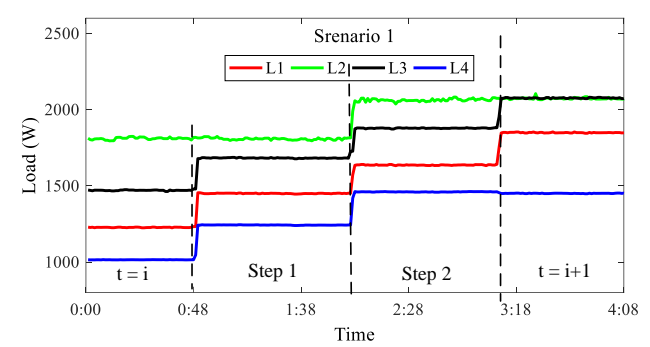

Figure 7.20: Load distribution in scenario1

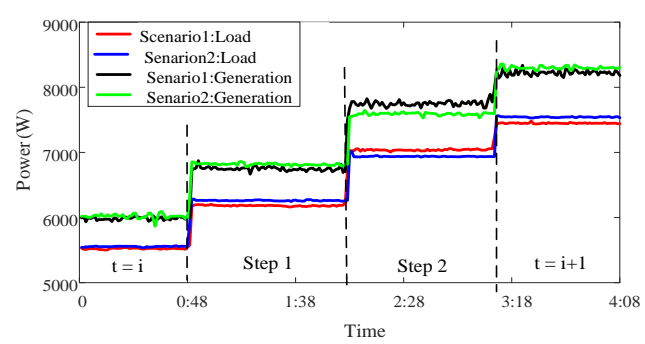

Figure 7.22: Total generation and load in both scenarios

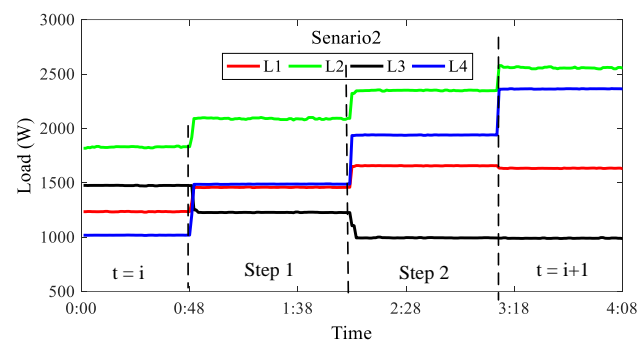

Figure 7.21: Load distribution in scenario2

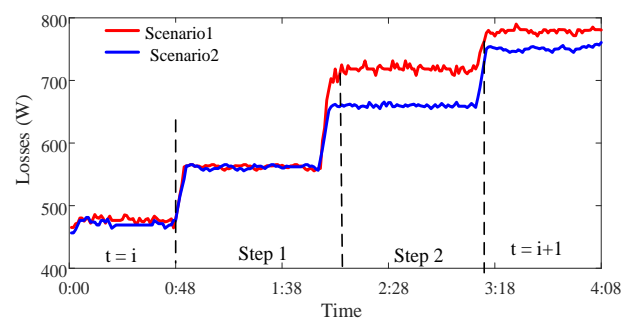

Figure 7.23: Active power losses in both scenarios

In this platform, a DDE is activated to transfer data between DIgSILENT PowerFactory (as the system simulator and optimization software), and LabVIEW (as the SCADA system). Software interoperability in this study is shown in Figure 7.19, where the optimization process controls the SCADA through the DDE. Although system loads are voltage-dependent, approximately each load step is around $248 \mathrm{~W}$, and each load has ten steps. To run the optimization, we consider that the charging rate of each EV is $248 \mathrm{~W}$, and therefore, each CSs can serve from 0 to 10 EVs. To get the numerical results, we run the system in two scenarios; In the first scenario (Scenario 1), the total load of the system is increasing from $5520 \mathrm{~W}$ to $7446 \mathrm{~W}$.

Table 7.4: EVs' distribution in scenario 1 and 2.

\begin{tabular}{|l|cccc|cccc|}
\hline CSS & \multicolumn{4}{|c|}{ SCENARIO 1 } & \multicolumn{4}{c|}{ SCENARIO 2 } \\
\hline STEP & $\mathbf{t}=\mathbf{i}$ & S1 & S2 & $\mathbf{t}=\mathbf{i}+\mathbf{1}$ & $\mathbf{t}=\mathbf{i}$ & S1 & S2 & $\mathbf{t}=\mathbf{i + 1}$ \\
CS1 & 5 & 6 & 7 & 8 & 5 & 6 & 7 & 7 \\
CS2 & 7 & 7 & 8 & 8 & 7 & 8 & 9 & 10 \\
CS3 & 6 & 7 & 8 & 9 & 6 & 5 & 4 & 4 \\
CS4 & 4 & 5 & 6 & 6 & 4 & 6 & 8 & 10 \\
SUM & 22 & 25 & 29 & 31 & 22 & 25 & 28 & 31 \\
\hline
\end{tabular}


The active power set-points for DG1 and DG2 are 1 and $1.5 \mathrm{~kW}$, respectively. The distribution of EVs at the beginning of this period $(t=i)$ is shown in Fig 20. The loads gradually change in two steps to reach their final values at $t=i+1$. Here, $\mathrm{S} 1$ and $\mathrm{S} 2$ stand for step1 and step2 in Table 7.4.

In the second scenario (Scenario 2), the system is run from exactly the previous starting point, but the same amount of load increase happens in the system by the different distribution of EVs among ICs as shown in Figure 7.21. The generation and load in both scenarios are shown in Figure 7.22, and the differences between these values are indicated in Figure 7.23, where the active power losses decrease from $789 \mathrm{~W}$ in scenario 1 to $752 \mathrm{~W}$ in scenario 2. The base distribution of EVs, along with their distributions in two steps of load variations in both scenarios are reported in Table 7.4. In both scenarios, the total number of EVs is increasing from $22($ at $t=i)$ to $31($ at $t=i+1)$ while the EVs' distribution between CSs is different. The optimal distribution (scenario 2) shows an increase in the number of EVs in CS2 and CS4 while it decreases the EVs from CS1 and CS3 comparing with scenario 1 . This redistribution leads to $4.67 \%$ of loss reduction in this system as shown in Figure 7.23.

\subsection{Summary}

In this chapter, an optimal power routing scheme between and within ICs was developed to minimize the power imbalance factor at the PCC, active power losses and voltage deviation indices in the grid-connected mode of an unbalanced hybrid AC-DC microgrid, where there are several independent ICs in the system. The effectiveness of the developed algorithm was confirmed through numerical results obtained from the simulation of the modified IEEE 13 bus system as a highly unbalanced hybrid AC-DC microgrid and IEEE 
34-bus test system as an unbalanced distribution system. Furthermore, to show the effect of the power routing between CSs on the active power losses reduction, a laboratory-based smart microgrid was established and examined. Numerical results demonstrated that the developed OPR scheme was highly successful to minimize the objective functions and smooth the voltage profile within the system. Consequently, optimal operation of unbalanced hybrid AC-DC microgrids would be possible when the developed OPR algorithm is implemented. More data and details have been reported in Appendix III. 


\section{Chapter 8 Real-Time Congestion Management in Power Markets}

In deregulated power systems, where a short period of service interruption causes extreme financial and social damages to customers and service providers, it is necessary to develop optimal intelligent algorithms to minimize unforeseen service interruptions due to unavoidable real-time contingencies. Nowadays, regarding the high implementation of communication infrastructure in smart power systems, as well as accurate sensors for a variety of purposes, it is possible to effectively collect and analyze real-time and synchronized data, run fast intelligent algorithms, and send control commands to controllers. Int his chapter, we propose adaptive RTCM method, which optimally employs adaptive thermal ratings of transmission lines to manage real-time congestions using all power system capabilities. This algorithm is considered as an essential ancillary service in a power market, where all generation companies and customers can participate. In this algorithm, a demand response program is modeled, and also a real-time hybrid optimization algorithm is developed to solve the RTCM problem aimed at finding the optimal solution during a short time span. Incorporating an adaptive artificial neural network along with a modified PSO algorithm is developed in this dissertation as a realtime hybrid optimization method. Numerical results demonstrate the advantages and effectiveness of this method from analyzing the modified 39-bus New England system.

\subsection{Nomenclature}

Table 8.1 shows parameters, functions and variables for the developed RTCM problem formulation. 
Table 8.1: Nomenclature for Chapter 8.

A. PARAMETERS

\begin{tabular}{|c|c|}
\hline $\boldsymbol{B}_{c i}$ & Coefficients for upper and lower bands in the $\mathrm{i}^{\text {th }}$ iteration of the PSO. \\
\hline$C_{g}$ & Energy bidding price for the $\mathrm{g}^{\text {th }}$ generator $(\$ / \mathrm{MW}-\mathrm{min})$ \\
\hline$C_{L}$ & Energy bidding price for the $\mathrm{L}^{\text {th }}$ load (\$/MW-min). \\
\hline$C_{p}$ & Conductor thermal capacity $\left(\mathrm{J} / \mathrm{kg}^{\circ}{ }^{\circ} \mathrm{C}\right)$. \\
\hline$D_{R}$ & Binary input indicating the demand response participation. \\
\hline$G S_{g}^{j}$ & Active power sensitivity of $\mathrm{j}^{\text {th }}$ line to the active power of the $\mathrm{g}^{\text {th }}$ generator. \\
\hline$I_{0}$ & The initial value of the conductor current (A). \\
\hline$I t_{i}$ & The $\mathrm{i}^{\text {th }}$ iteration in the PSO. \\
\hline$I t_{\operatorname{Max}}$ & Maximum number of iterations in the PSO. \\
\hline$K_{T}^{j}$ & Active power sensitivity of $j^{\text {th }}$ line to the phase shifting variation of the $T^{\text {th }}$ PST. \\
\hline$L S_{L}^{j}$ & Active power sensitivity of $\mathrm{j}^{\text {th }}$ line to the active power variation of the $\mathrm{L}^{\text {th }}$ load. \\
\hline $\boldsymbol{m}$ & Mass per unit length of the conductor $(\mathrm{kg} / \mathrm{m})$. \\
\hline$N g$ & Number of generators. \\
\hline$N L$ & Number of loads. \\
\hline$N_{\text {line }}$ & Number of transmission lines. \\
\hline$N p$ & Number of PSTs. \\
\hline$P_{g}^{0}$ & Initial active power set point of the $\mathrm{g}^{\text {th }}$ generator $(\mathrm{MW})$. \\
\hline$P_{g}^{M a x}$ & Maximum operational limit for the $\mathrm{g}^{\text {th }}$ generator's active power (MW). \\
\hline$P_{g}^{M i n}$ & Minimum operational limit for the $\mathrm{g}^{\text {th }}$ generator's active power (MW). \\
\hline$P_{j}^{0}$ & Post-contingency active power flow in the $\mathrm{j}^{\text {th }}$ line $(\mathrm{MW})$. \\
\hline$P_{j}^{L T R}$ & Maximum active power of the $\mathrm{j}^{\text {th }}$ line at the LTR conductor current (MW). \\
\hline$P_{j}^{M a x}$ & Maximum value for the active power of the $\mathrm{j}^{\text {th }}$ line $(\mathrm{MW})$ \\
\hline$P_{j}^{S T R}$ & Maximum active power of the $\mathrm{j}^{\text {th }}$ line at the STR conductor current (MW). \\
\hline$P_{L}^{0}$ & Initial active power of the $\mathrm{L}^{\text {th }}$ load (MW). \\
\hline$P_{L}^{\operatorname{Max}}$ & Maximum amount of the $\mathrm{L}^{\text {th }}$ load (MW). \\
\hline$P_{L}^{M i n}$ & Minimum amount of the $\mathrm{L}^{\text {th }}$ load (MW). \\
\hline$P_{\text {Size }}$ & Population size of the PSO. \\
\hline $\boldsymbol{R}$ & Conductor resistance $(\mathrm{ohm} / \mathrm{m})$ \\
\hline$t_{c c}$ & Actual congestion clearing time (minute). \\
\hline$T_{0}$ & The initial conductor temperature $\left({ }^{\circ} \mathrm{C}\right)$ \\
\hline$T_{c c}^{M a x}$ & Maximum time span for the congestion clearing time (minute). \\
\hline$T_{\operatorname{Max}}$ & Maximum conductor temperature $\left({ }^{\circ} \mathrm{C}\right)$ \\
\hline$T_{s}$ & The time required to solve the optimization problem (s). \\
\hline$R_{g}^{D o w n}$ & Ramp down rate of the $\mathrm{g}^{\text {th }}$ generator (MW/minute). \\
\hline$R_{g}^{U p}$ & Ramp up rate of the $\mathrm{g}^{\text {th }}$ generator (MW/minute). \\
\hline$\Delta \Phi_{T}^{M a x}$ & Maximum achievable phase shifting for $\mathrm{T}^{\text {th }} \operatorname{PST}\left(^{\circ}\right)$. \\
\hline$\Delta \Phi_{T}^{M i n}$ & Minimum achievable phase shifting for $\mathrm{T}^{\text {th }} \operatorname{PST}\left({ }^{\circ}\right)$ \\
\hline
\end{tabular}




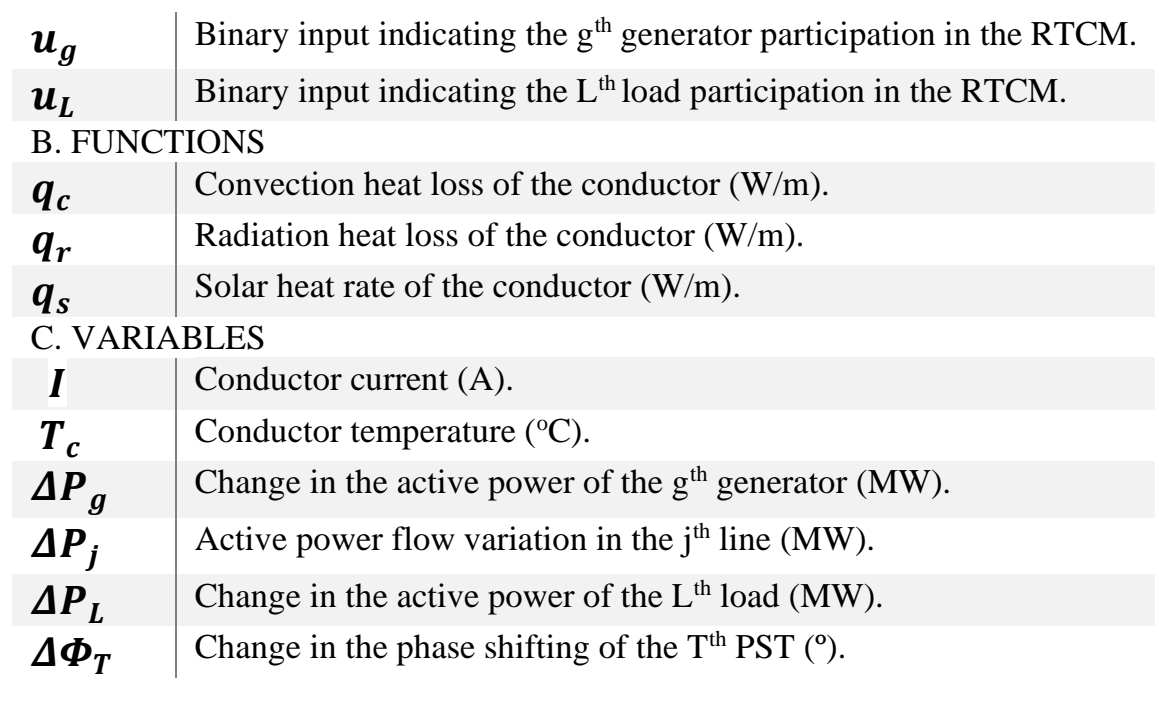

\subsection{RTCM Formulation}

In [101], a comprehensive RTCM formulation has been presented considering generators' rescheduling and load shedding tools. In [102], the authors studied the effect of the PST on the RTCM problem. In this dissertation, a market-based RTCM formulation is presented, which considers the DR instead of load shedding, as shown by (8-1).

$$
\min \left(\sum_{g=1}^{N g} u_{g} \cdot C_{g} \cdot\left|\Delta P_{g}\right|+D_{R} \cdot \sum_{L=1}^{N L} u_{L} \cdot C_{L} \cdot\left|\Delta P_{L}\right|\right)
$$

Subject to:

$$
\begin{gathered}
\left(P_{j}^{0}+\sum_{g=1}^{N g} u_{g} \cdot G S_{g}^{j} \cdot\left|\Delta P_{g}\right|+D_{R} \cdot \sum_{L=1}^{N L} u_{L} \cdot L S_{L}^{j} \cdot\left|\Delta P_{L}\right|+\sum_{T=1}^{N p} K_{T}^{j} \cdot \Delta \Phi_{T}\right) \\
\leq P_{j}^{\text {Max }}, \quad j=1,2,3, \ldots, N_{\text {line }} \\
\left(P_{g}^{\text {Min }}-P_{g}^{0}\right) \leq \Delta P_{g} \leq\left(P_{g}^{\text {Max }}-P_{g}^{0}\right), \quad g=1,2,3, \ldots, N g \\
\left(R_{g}^{\text {Down }} \cdot t_{c c}\right) \leq \Delta P_{g} \leq\left(R_{g}^{U p} \cdot t_{c c}\right), \quad g=1,2,3, \ldots, N g \\
\left(P_{L}^{\text {Min }}-P_{L}^{0}\right) \leq \Delta P_{L} \leq\left(P_{L}^{\text {Max }}-P_{L}^{0}\right), \quad L=1,2,3, \ldots, N L \\
\Delta \Phi_{T}^{\text {Min }} \leq \Delta \Phi_{T} \leq \Delta \Phi_{T}^{\text {Max }}, \quad T=1,2,3, \ldots, N p
\end{gathered}
$$




$$
\sum_{g=1}^{N g} u_{g} \cdot \Delta P_{g}-D_{R} \cdot \sum_{L=1}^{N L} u_{L} \cdot \Delta P_{L}=0
$$

Where,

$$
t_{c c}=T_{c c}^{M a x}-T_{s}
$$

Equation (8-2), shows the transmission line active power due to generation rescheduling, DR and PSTs' phase shifting. The sensitivity factors in (8-2), are defined by (8-9)-(8-11).

$$
\begin{gathered}
G S_{g}^{j}=\frac{\Delta P_{j}}{\Delta P_{g}} \quad, j=1,2,3, \ldots, N_{\text {line }}, g=1,2,3, \ldots, N_{g} \\
L S_{L}^{j}=\frac{\Delta P_{j}}{\Delta P_{L}} \quad, L=1,2,3, \ldots, N_{\text {load }} \\
K_{T}^{j}=\frac{\Delta P_{j}}{\Delta P_{T}} \quad, T=1,2,3, \ldots, N_{p}
\end{gathered}
$$

The calculation method for these sensitivity factors is one of the most important parts of the RTCM problem. The calculation method for the $G S_{g}^{j}$ and $L S_{L}^{j}$ is based on power flow equations by neglecting P-V coupling and has been described in detail in [104]. Furthermore, the calculation method for the $K_{T}^{j}$ has been presented in [102]. Sensitivities are calculated very fast in the post-contingency condition using the admittance matrix elements, voltage magnitude, and phase angle of system buses. In (8-3), operational limits for the generators' variation have been indicated while the real-time capability of generators for rescheduling programs regarding their up and down ramp rates have been demonstrated in (8-4). Equation (8-5) and (8-6) show loads' variation limits in the DR and PSTs' variation limits in the RTCM problem. A very low solution time for the RTCM problem is the main advantage of this formulation because it does not include the power flow equations in optimization iterations. As a result, finding a fast solution for the RTCM 
problem would be possible, which is critical for real-time applications. Although (8-7) estimates the output of the slack generator, the exact value is obtained after a complete AC power flow solution considering the outputs of the RTCM problem for all generators, loads, and PSTs. The feasibility of the solution regarding voltage limits, actual line loadings, and stability issues is evaluated in this step before sending the control commands to all participants in the RTCM market.

If the solution is not feasible, the algorithm tries to find another solution for the problem by introducing more restricted values for optimization constraints. In (8-8), the congestion clearing time $\left(t_{c c}\right)$ is calculated, which is defined as the actual time to remove the congestion from the transmission system securely [104]. The optimization solution time $\left(T_{S}\right)$, is the time required to solve the optimization problem to find a feasible set of control commands. Since we have defined the maximum value for the congestion clearing time $\left(T_{c c}^{M a x}\right)$, the actual value for clearing the congestion $\left(t_{c c}\right)$ is calculated by subtracting the $T_{s}$ from the $T_{c c}^{M a x}$ as shown in (8-8).

Generally, selecting appropriate values for both $T_{c c}^{M a x}$ and $T_{S}$ is very important because they could significantly affect the RTCM final solution. In the next section, the calculating method for the $T_{c c}^{M a x}$ is introduced and as discussed in [104], usually a constant value for the $T_{S}$ is selected regarding the solver's capabilities.

\subsection{Short and Emergency Thermal Ratings}

The maximum value for the congestion clearing time $\left(T_{c c}^{M a x}\right)$ is determined regarding the short and emergency thermal rates of congested lines [101]. Based on the concept of the thermal inertia of conductors, different current rates prevail over the specific time spans in different weather conditions. 


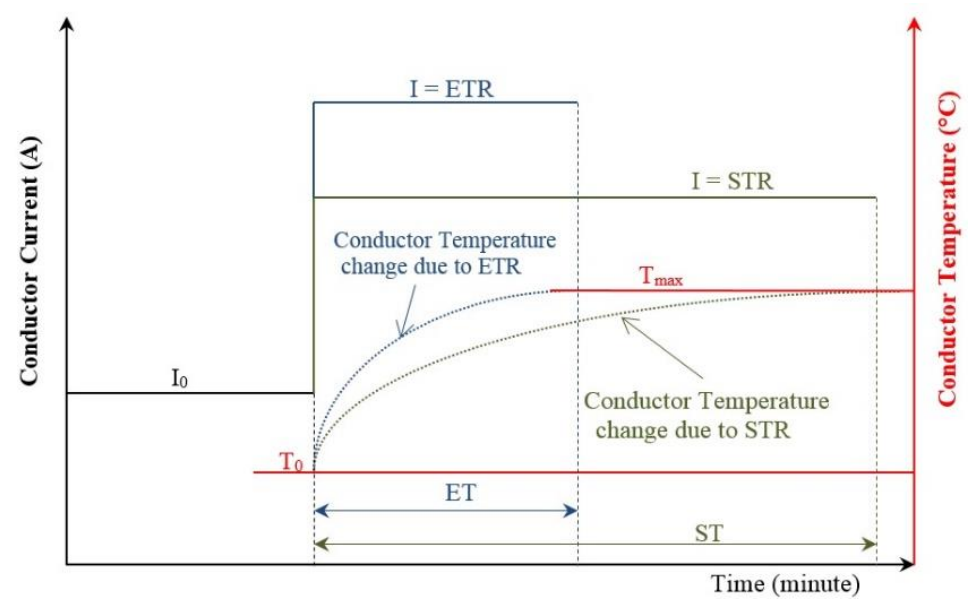

Figure 8.1: Determination of STR and ETR rates [101].

Figure 8.1 shows the STR and ETR for a typical transmission line. Based on thermal rating strategies and the thermal inertia of the conductor, the conductor current could be increased for a specific time interval before the conductor temperature reaches its maximum value. These time intervals are defined as the ST and ET. Using the worst weather condition for thermal rating calculations will lead to conservative rates, which are sometimes much lower than the actual thermal capacity of a transmission line. These rates are called static thermal rates, and as expected, the optimal usage of installed capacity will not be available because of these conservatisms. If the real-time weather condition is used to calculate thermal ratings, dynamic thermal ratings would be available which are usually higher than static rates. Since this method uses the real-time weather condition, it may oscillate significantly during the operation time. Therefore, quasi-dynamic thermal rates of transmission lines have been introduced in [101], which are based on the short-term prediction of the weather condition and are more stable than dynamic rates. Although using dynamic and quasi-dynamic rating strategies lead to a higher transmission line thermal capacity, for the sake of simplicity, the static thermal rating strategy is used in this dissertation. From the results in [101], it can be seen that the STR and ETR are around 
$118 \%$ and $147 \%$ of the LTR for a transmission line, respectively. These rates are valid for 15 and 5 minutes, respectively (Let $\mathrm{ST}=15$ and $\mathrm{ET}=5 \mathrm{~min}$ ).

Hence, the $T_{c c}^{M a x}$ would be 15 minutes if the loading of the congested line is between $100 \%$ to $118 \%$ (STR region) or 5 minutes if the line's loading is between $118 \%$ to $147 \%$ (ETR region). For all congestions, which are more than the ETR level, an instantaneous relay tripping happens. In this dissertation, we optimally use these short and emergency thermal rates as adaptive thermal rates of conductors by introducing a two-step RTCM method.

\subsection{Two-Step RTCM Method}

When the line current is in the ETR region, the optimization constraints are more restricted due to the lower value of $T_{c c}^{M a x}$ (5 minutes). As a result, the RTCM cost may increase significantly. Furthermore, in some cases, the solver may fail to find a feasible solution. In this dissertation we address these issues by proposing two-step RTCM method regarding adaptive thermal rates of conductors as shown in Figure 8.2. This method divides the RTCM problem into the two-step optimization problem for congestions in the ETR region. It should be noted that when a congestion occurs, the solver tries to find a feasible solution for the RTCM problem during the optimization solution time $\left(T_{s}\right)$. Before finding a feasible solution, there are not any control commands and therefore, any reaction from generators, loads and transformers during this period. After passing the solution time (at the end of the solution time period), by finding a feasible solution, control signals are sent to generators, loads, and transformers, and they start to react to the received commands. 


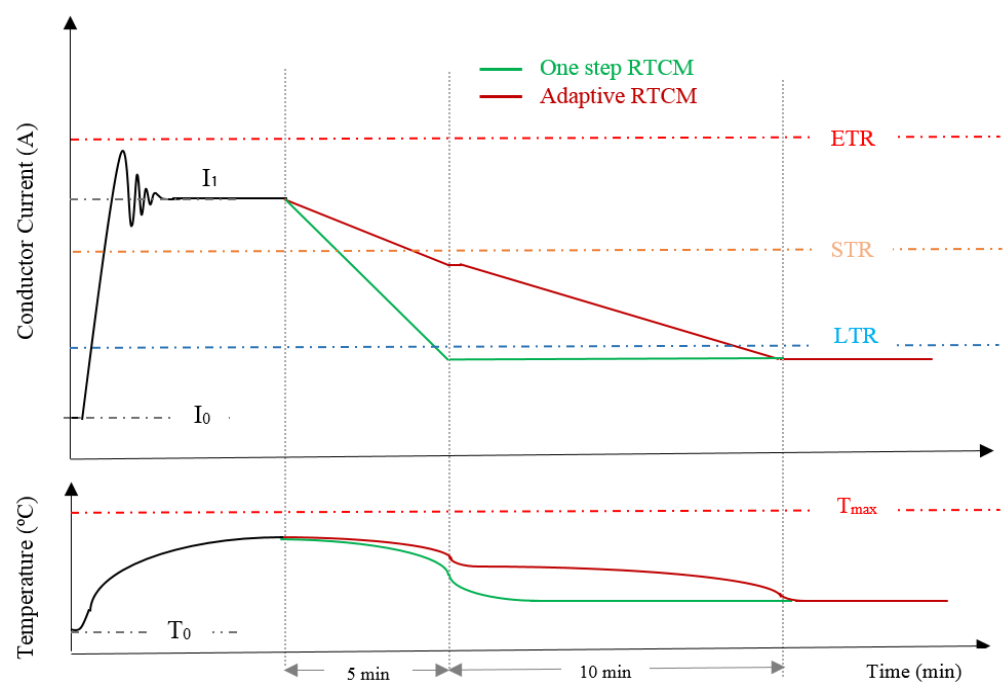

Figure 8.2: The concept of adaptive RTCM method.

Usually transformers' tap changers and demand responses are much faster than generators' responses and as a result, generators' response time may continue up to end of actual clearing time period $\left(t_{c c}\right)$ regarding the rescheduling commands and ram rates.

At the first step, the algorithm manages the congestion in such a way that conductor current decreases to its STR level during the ET (set $T_{c c}^{M a x}=5 \min$ and $P_{j}^{M a x}=P_{j}^{S T R}$ ). The next step is to remove the congestion from the system in the STR region. At this point, it is necessary to calculate the $T_{c c}^{M a x}$. Since we have been in the ETR region for 5 minutes, in the second step the $T_{c c}^{M a x}$ is calculated as below:

$$
T_{c c}^{M a x}=S T-E T=15-5=10 \mathrm{~min}
$$

Furthermore, as shown in Figure 8.2, by reaching the STR level, for a short time span, the conductor current remains constant. It is the time required to solve the optimization problem $\left(T_{s}\right)$. Afterward controllers try to reduce the congested line's current to its LTR.

Generally, using the IEEE Standard 738 [192] and equation (8-13), which is known as the transient (non-steady-state) thermal equation, it is possible to calculate the time required to reach the maximum conductor temperature for those level of currents which are 
more than LTR level.

$$
q_{s}+R I^{2}=q_{c}+q_{r}+m C_{p} \frac{d T_{c}}{d t}
$$

In fact, $(8-13)$ can be rewritten as (8-14) to find the rate of temperature change due to a new value of the conductor current.

$$
\frac{d T_{c}}{d t}=\frac{1}{m C_{p}}\left(q_{s}+R I^{2}-q_{c}-q_{r}\right)
$$

The conductor temperature is increasing step by step and in each one, the rate of temperature change is calculated by updated values of $q_{s}, R, q_{c}$ and $q_{r}$ which are affected by the new value of $T_{c}$. This process is time consuming and will result in different time intervals for different current rates to reach the maximum conductor temperature. Although calculating this time will lead to having more $T_{c c}^{M a x}$ in the RTCM problem, from the practical point of view, it is not possible to change the overcurrent relay settings continuously. Especially in the post-contingency condition, when we quickly need to guide the system to another safe operating point. Therefore, pre-defined values for the ST and ET should be used periodically based on thermal rating calculations.

\subsection{Real-Time hybrid optimization algorithm}

In real-time optimization problems, fast solution methods are needed. Because of this fact, solvers usually try to find the first feasible solution using classical methods, which are used for multivariable and both Equality and inequality constraints (ex. Lagrange multiplier method.). These methods lead to a set of nonlinear simultaneous equations that may be difficult to solve. Furthermore, there are some heuristic search algorithms which are used for optimization problems, where they can search for the global solution instead of the local one. For example, the PSO, genetic, Tabu search algorithm, etc. These 
algorithms are usually used for nonlinear or complicated problems where classical methods fail to find a feasible or global solution. Although these methods have many advantages in optimization problems, there are some drawbacks when these methods are used. The most important issue is the solution time, which is often much higher than what is required for a classical method. Therefore, in real-time optimization problems, it is impossible to use heuristic methods as they are used in offline optimization purposes.

Determination of heuristic search algorithms' parameters is another important issue that should be noticed when these algorithms are utilized. Generally, parameters of heuristic methods are determined based on trial and error, and there are different indexes that could be implemented to stop the search process, but in the real-time application, using the trial and error method is not acceptable. In this dissertation, a hybrid real-time optimization method using a modified PSO algorithm and an AANN is developed to find an optimal feasible solution for the RTCM problem in a pre-defined time span.

\subsubsection{Modified PSO Algorithm}

The main concept of the PSO algorithm has been discussed in [193]. Here, some modifications are suggested to improve the performance of the PSO algorithm in such a way that it could be used for the RTCM problem in a specific time interval. Since some of PSO variables in our RTCM problem are generation and load values, imposing a huge rescheduling aimed at minimizing the RTCM cost without considering the power flow equations, may lead to an unfeasible solution after running a full $\mathrm{AC}$ power flow at the end of the optimization process. In other words, the probability of finding a feasible solution for the RTCM problem may decreases if variables are chosen randomly within their nominal limits. 


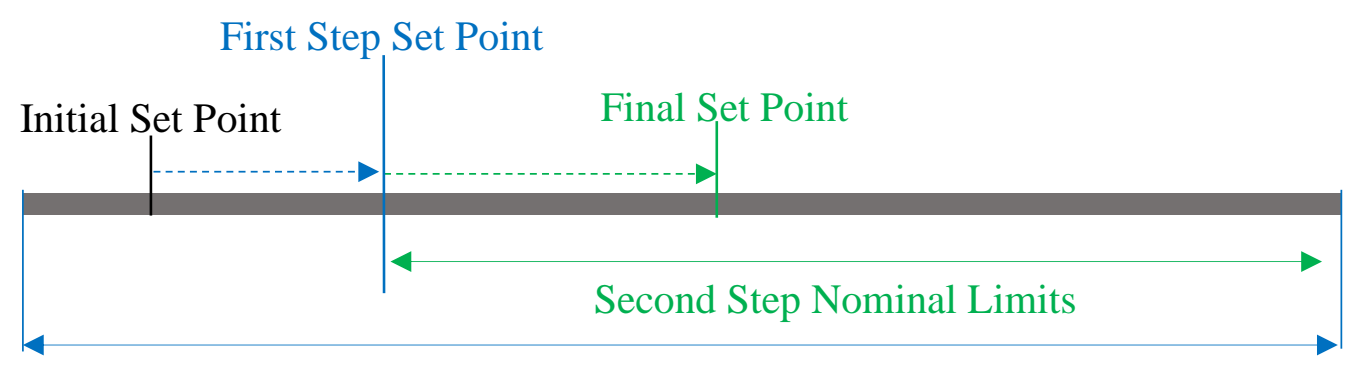

First Step Nominal Limits

Figure 8.3: Nominal band limit determination in the adaptive RTCM algorithm

It should be noted that in the offline process of the PSO algorithm, we can increase the population size and iteration number in such a way that all solution space could be investigated. But, in real-time applications, where we need to find a feasible solution very fast, it is necessary to guide the algorithm to a feasible solution by controlling variables' limits.

Therefore, we set the post-contingency point as one of the population members at the first iteration because it is a feasible solution for the power flow problem. However, it does not satisfy the problem's constraints. The PSO algorithm will create other populations randomly and will analyze the objective function and constraints for each of them. In this method, the operating point could slowly move to an optimal point while it has the freedom to search for all the solution space.

\subsubsection{Band Coefficient Definition}

Band coefficients are defined to ensure us about searching in proper solution space. Limiting the solution space in the first iteration of the PSO and releasing upper and lower bands in the next steps will lead the PSO to move from the post-contingency operation point to an optimal point gradually. In this method, variables can only move from their existing point slowly regarding the iteration number of the algorithm. For example, assume 
that we have ten iterations. At the first iteration, random values for variables could be selected between $90 \%$ to $110 \%$ of their initial values. In the next iteration, the PSO algorithm would be able to devote random values to them within $\pm 20 \%$ of their permitted variation areas. At the final iteration, all limits are released to their nominal values. Coefficients for upper and lower bands are calculated as below:

$$
B C_{i}=\frac{I t_{i}}{I t_{\text {Max }}} \quad, i=1,2,3, \ldots, I t_{\text {Max }}
$$

In fact, the PSO uses these coefficients for constraints in equations (8-3)-(8-6) as below:

$$
\begin{gathered}
B C_{i} \cdot\left(P_{g}^{\text {Min }}-P_{g}^{0}\right) \leq \Delta P_{g} \leq B C_{i} \cdot\left(P_{g}^{\text {Max }}-P_{g}^{0}\right), g=1,2,3, \ldots, N g \\
B C_{i} \cdot\left(R_{g}^{\text {Down }} \cdot t_{c c}\right) \leq \Delta P_{g} \leq B C_{i} \cdot\left(R_{g}^{U p} \cdot t_{c c}\right), g=1,2,3, \ldots, N g \\
B C_{i} \cdot\left(P_{L}^{\text {Min }}-P_{L}^{0}\right) \leq \Delta P_{L} \leq B C_{i} \cdot\left(P_{L}^{\text {Max }}-P_{L}^{0}\right), L=1,2,3, \ldots, N L \\
B C_{i} \cdot \Delta \Phi_{T}^{\text {Min }} \leq \Delta \Phi_{T} \leq B C_{i} \cdot \Delta \Phi_{T}^{\text {Max }}, T=1,2,3, \ldots, N p
\end{gathered}
$$

\subsubsection{Variables' direction control}

At the second step of the RTCM, variables' variation direction for generators and loads should be the same as what was in step one. In other words, if at the first step of the RTCM, the final decision is to increase the $\mathrm{g}^{\text {th }}$ generator's active power, at the second step, the algorithm can only follow this increment or stop changing this value. Hence, after the first step of the RTCM, we define new nominal band limits for variables regarding their direction in the first step. Figure 8.3 shows these limits for a sample generator or load.

\subsubsection{Adaptive Artificial Neural Network}

The PSO convergence depends on the problem structure and the number of variables, objective function, and optimization constraints. The main two parameters which play a vital role in the PSO algorithm convergence are population size and the number of 


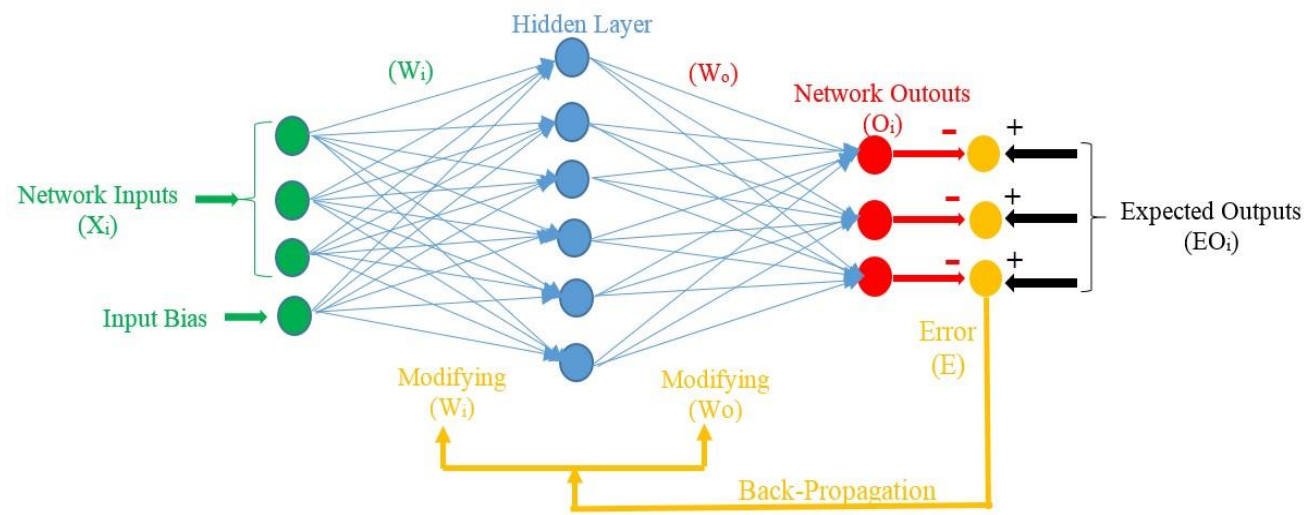

Figure 8.4: Architecture of the back-propagation model for the AANN.

iterations, which are usually determined by the trial and error method. However, in the RTCM problem, we need to estimate appropriate numbers for these two parameters in such a way that the PSO algorithm could converge to a feasible solution in pre-defined solution time. The network configuration in the post-contingency condition, generators, and loads value is the most important data which could impact the RTCM optimization problem. Furthermore, it also has a significant effect on the optimization result. Therefore, the PSO parameters would also be affected by these data. On the other hand, there are too many contingency situations which could be occurred in the power system, for example, N-1, N2 , etc. At different load levels and generation schemes. It means that predicting and simulating all of contingencies are not practically possible to determine the PSO parameters for each one. Therefore, we need to use an algorithm to estimate the PSO parameters according to the contingency situation.

In this dissertation, an AANN is developed as a dynamic tool to estimate the PSO parameters in the RTCM problem. Figure 8.4 shows the structure of this neural network. The configuration of the transmission system along with generation and load schemes are defined as input parameters $\left(\mathrm{X}_{\mathrm{i}}\right)$ while the PSO population size and iteration number would be outputs of the AANN $\left(\mathrm{O}_{\mathrm{i}}\right)$. Furthermore, in each congestion case, we need to know that 
if the DR is needed for this case or not, therefore, we define the DR also as an output of the AANN.

Since it is impossible to predict real-time contingencies in a control area of a power system, we need to use the adaptive algorithm in the ANN to train the network by new contingencies in different network configurations, generation, and load patterns. In this dissertation, a feed-forward, back-propagation algorithm is used to estimate the PSO parameters and the DR participation for the RTCM problem. For this purpose, a set of contingencies is selected, and based on the trial and error mechanism, the PSO parameters and demand response participation are determined for the RTCM problem. Regarding the number of transmission lines, generators and loads, the number of inputs, outputs and hidden layer neurons are created. After that, the ANN weights $\left(W_{i}\right.$ and $\left.W_{o}\right)$ are initialized randomly and network training is started. If the power system deals with a new contingency condition (new input/output data) in the real-time operation, that situation is adapted to the network considering the exciting weights from the training process. The network training stop criteria are reaching to the maximum number of iterations, or the determined RMSE value.

\subsubsection{Training process}

For the training purpose, we identify the network configuration using binary values for transmission lines for generators' set points and loads' levels, and normalized inputs are used by dividing them by the largest generation capacity in the network. The value of the

$T_{c c}^{M a x}$ is equal to 5, 10 or 15 minutes based on the contingency condition. We define $0,0.5$ and 1 for these values respectively as normalized inputs. The output data are population size and maximum iteration number which are also normalized by dividing by a base value 
(in this dissertation, the output-base-value is equal to 100) along with a binary value for the DR which shows the demand response participation. The number of neurons in the input and output layers is equal to the number of inputs and outputs, respectively.

The training process is started by creating random values for inputs, outputs and bias weights and propagating inputs and outputs through the network layers. Then, the sum of errors (errors between network outputs $\left(\mathrm{O}_{\mathrm{i}}\right)$ and expected outputs $\left(\mathrm{EO}_{\mathrm{i}}\right)$ ) is calculated and the input and output data will be injected into the network again by using the modified values of the weights. By continuing this process, better performance of the ANN would be achievable. The mathematical model of this process has been clearly described in [194]. Therefore, we do not repeat in this dissertation. Reaching a pre-defined threshold error or a maximum number of iterations are two criteria for stopping the training process. The weights would be able to adapt their values while new contingencies are reported based on weights adaption equations [194].

\subsection{The developed adaptive RTCM Algorithm}

Figure 8.5 shows the developed adaptive RTCM algorithm containing a two-step optimization process using the developed hybrid real-time optimization method. As can be seen in this flowchart, due to the congestion occurrence, two parallel processes are started: the first one is the network configuration detection as input data for the AANN, and the second one is the calculation of sensitivities, which is depended on the post-contingency operating point. The outputs of these two processes are imported to the PSO program. After running the modified PSO algorithm, in each iteration, stopping criteria are assessed, and if they are not satisfied, the algorithm goes to the next iteration. By reaching one of the stopping indexes or the maximum number of iterations, the feasibility of the PSO solution 


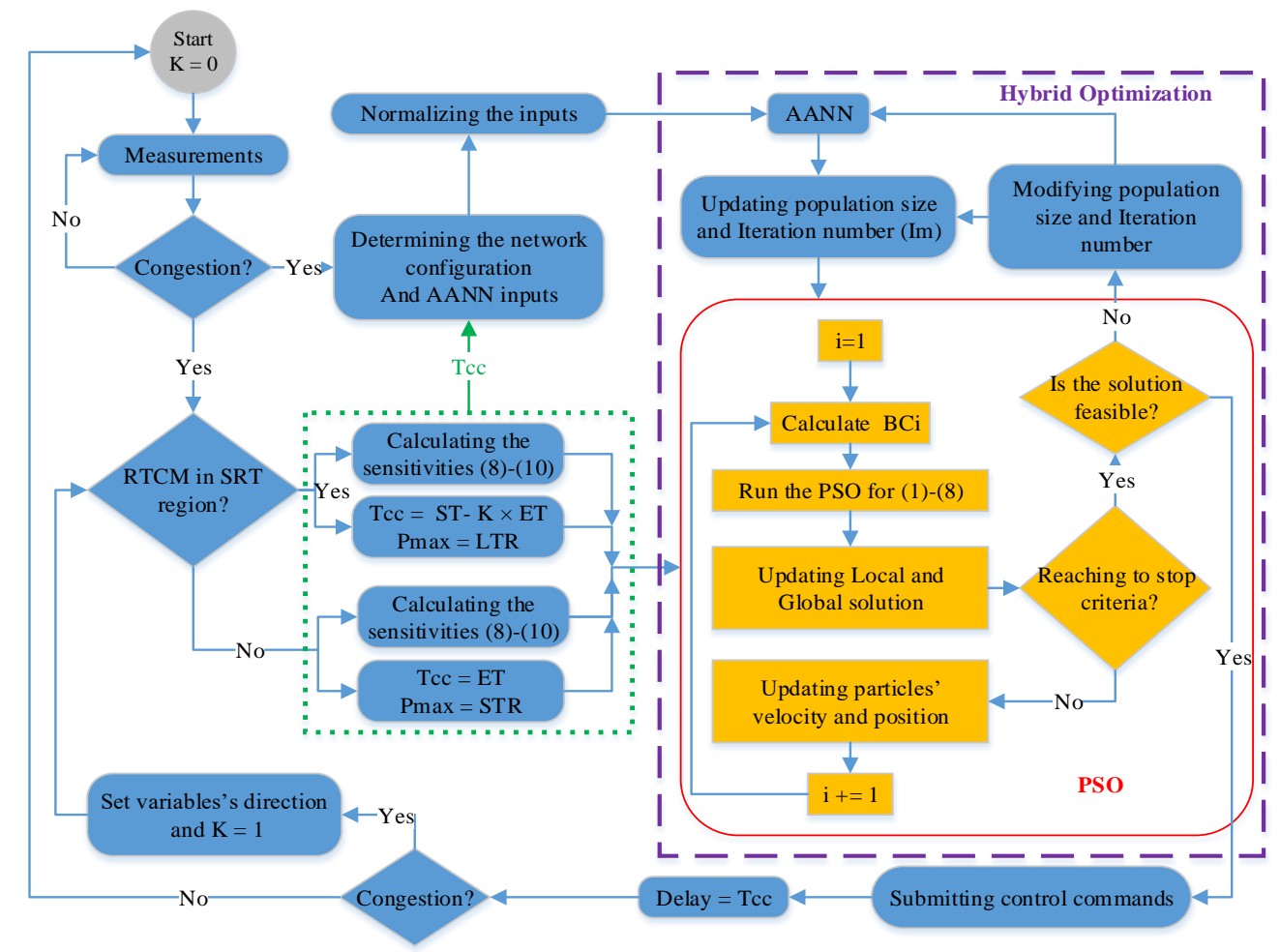

Figure 8.5: The developed adaptive RTCM algorithm.

is checked by solving a full AC power flow problem, including all rescheduling, demand responses, and PSTs' tap positions. If the solution is feasible, the control commands will be sent to the power system components. At this point, all participated components, including PSTs, loads, and generators, are triggered to change their setpoints based on the received control signals. It should be noted that automatic control of generation, load and transformers taps is required for implementing this algorithm as an essential and uninterruptible ancillary service.

After sending the first command set, a time delay, which is equal to the, is imposed on the algorithm before checking the transmission congestion situation because the RTCM process needs this time to reach its final operating point. Afterward, transmission system congestion is rechecked; if there is no congestion, the RTCM has been finished successfully. Otherwise, the RTCM goes to the second step, and the process will be 
repeated by a new value of clearing time and sensitivity factors. Hence, the developed adaptive RTCM algorithm follows the instructions for step one $(K=0)$ and two $(K=1)$ to remove the congestion optimally.

\subsection{Numerical Results}

In this dissertation, the modified 39-bus New England test system has been selected as our test system [102]. Two PSTs have been located in this system, as shown in Figure 8.6. Bid prices for rescheduling $(\mathrm{Cg})$ and generators' data have been reported in Table 8.2. To minimize the DR participation in the RTCM problem and, as a result, minimize the total RTCM cost, we assume that all loads are biding 100 (\$/MW-min). The Limits of Variation for each load are between $70 \%$ to $120 \%$ of its initial value. In this system, there are 34 transmission lines, 10 generation units, 21 loads, and 2 PSTs. Furthermore, we also have a defined value for the $T_{c c}^{M a x}$, therefore, there are 68 inputs for the ANN while only 3 outputs will be reported for each contingency case.

The number of neurons in the hidden layer is 87 and the stopping criteria for the ANN training is defined by $2 \times 10^{4}$ of iterations or RMSE $<1 \%$. Power system modeling, sensitivities, and power flow calculations were done using DIgSILENT PowerFactory 2017. The modified PSO algorithm was also developed in this software using the DPL. The AANN development and training were done in MATLAB and a data exchange framework between these tools has been created to test the developed RTCM method. Since DIgSILENT PowerFactory has the capability to monitor and run an actual power system using its engine mode, the practical results for actual power systems could be obtained using the suggested framework. 
Table 8.2: Generators' data for the modified 39-bus New England system.

\begin{tabular}{|l|c|c|c|c|c|c|c|c|c|c|}
\hline Unit & G1 & G2 & G3 & G4 & G5 & G6 & G7 & G8 & G9 & G10 \\
\hline $\mathrm{C}_{\mathrm{g}}(\$ / M W-m i n)$ & 0 & 30 & 25 & 20 & 10 & 35 & 40 & 30 & 50 & 60 \\
\hline $\mathrm{R}_{\mathrm{g}}^{\mathrm{Up}}=-\mathrm{R}_{\mathrm{g}}^{\text {Down }}(\mathrm{MW} / \mathrm{min})$ & 30 & 9 & 9 & 9 & 8 & 6 & 8 & 8 & 8 & 9 \\
\hline $\mathrm{P}_{\mathrm{g}}^{\text {Max }}(\mathrm{MW})$ & 8500 & 595 & 680 & 680 & 510 & 680 & 595 & 595 & 850 & 850 \\
\hline $\mathrm{P}_{\mathrm{g}}^{0}(\mathrm{MW})$ & 1036 & 590 & 650 & 632 & 508 & 650 & 560 & 540 & 830 & 250 \\
\hline $\mathrm{P}_{\mathrm{g}}^{\text {Min }}(\mathrm{MW})$ & 0 & 150 & 200 & 200 & 140 & 200 & 150 & 150 & 250 & 0 \\
\hline
\end{tabular}

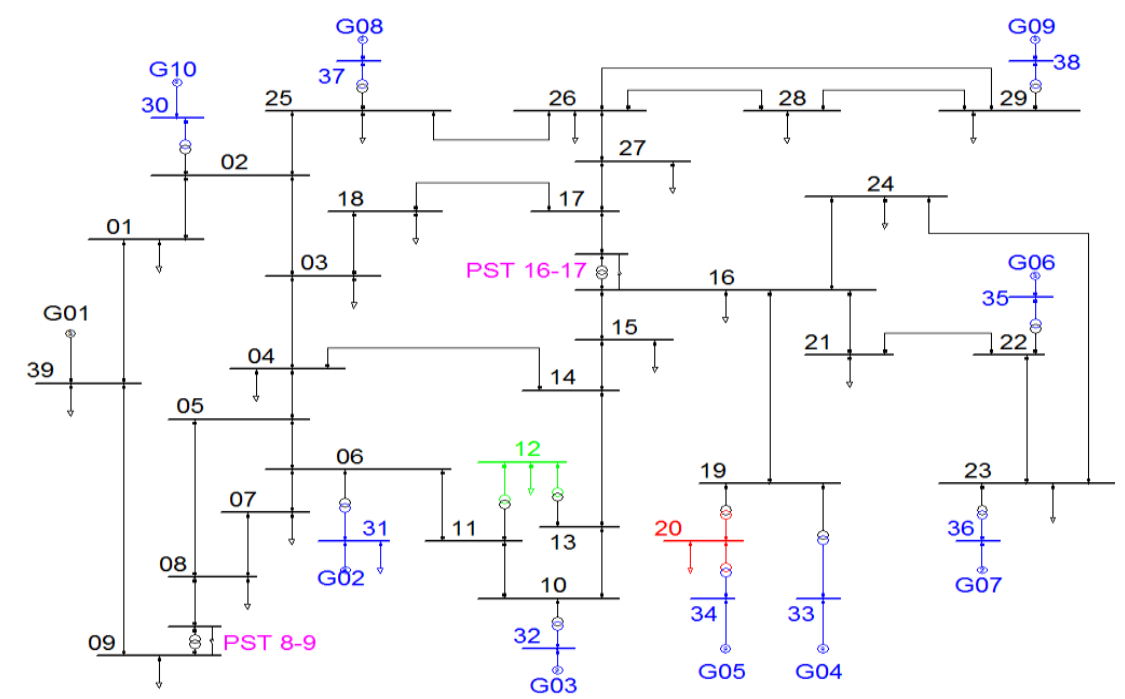

Figure 8.6: The modified 39-bus New England system with two PSTs [102].

\subsubsection{Training the ANN}

For the ANN training purpose, 217 contingencies that caused different congestions in the STR or ETR regions were selected and simulated considering different generation schemes and load levels, as reported in Table 8.3. For each of them, the PSO parameters were determined based on the trial and error mechanism aimed at finding the optimal feasible solution during the maximum allowed solution time $\left(T_{s}=10 \mathrm{sec}\right.$. $)$. After that, another two contingencies have been simulated in a heavily loaded system to evaluate the performance of the ANN and the adaptive RTCM method.

Table 8.3: Training data for the ANN.

\begin{tabular}{|c|c|c|c|}
\hline Load Level & Generation (MW) & Contingency type & Number of contingencies \\
\hline \multirow{2}{*}{ High } & \multirow{2}{*}{6246.48} & $\mathbf{N}-1$ & 34 \\
\cline { 3 - 4 } & \multirow{2}{*}{ Medium } & $\mathbf{N}-2$ & 50 \\
\hline \multirow{2}{*}{ Light } & \multirow{2}{*}{3747.18} & $\mathbf{N}-1$ & 34 \\
\cline { 3 - 4 } & & $\mathbf{N}-2$ & 35 \\
\cline { 3 - 4 } & $\mathbf{N - 1}$ & 34 \\
\hline
\end{tabular}




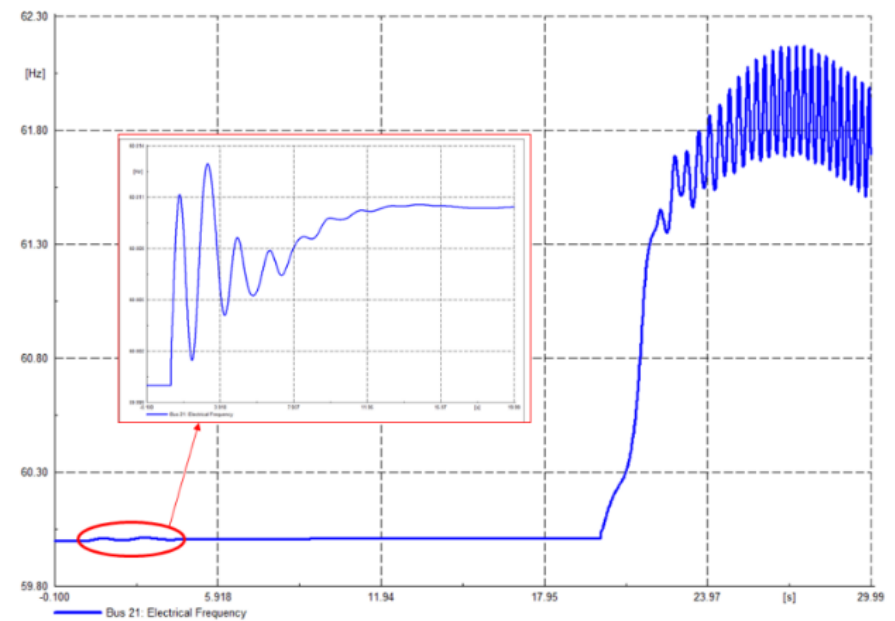

Figure 8.7: Instability due to the line $06-07$ outage in scenario 1.

\subsubsection{Scenario 1}

Like the first scenario, the outage of the line 05-08 due to a three-phase short circuit and tripping this line is studied. After this contingency, the transmission system will be congested by $37.30 \%$ of overload in the line $06-07$. This is a critical situation for the system because of instability and cascading outage after tripping this congested line. Figure 8.7 shows the system frequency due to the line $05-08$ outage (at $t=2 \mathrm{sec}$.) and the outage of the line $06-07$ due to its overloading (at $t=20 \mathrm{sec}$.). After the first outage, the system is still stable, but after the second one, the system would be unstable. Furthermore, from the static point of view, seven transmission lines will be overloaded in their ETR level and will be tripped instantaneously.

Therefore, the blackout is highly probable if the system operator does not run the RTCM algorithm or if the algorithm cannot manage the congestion securely. Table 8.4 reports the results for both the one-step and adaptive (two-step) RTCM algorithm. From this table, it can be concluded that the hybrid optimization method has been highly successful in this case regarding both PSO parameters estimation and the final solution. 
Table 8.4: RTCM results in scenario 1.

\begin{tabular}{|c|c|c|c|c|}
\hline \multirow{2}{*}{\multicolumn{2}{|c|}{$\begin{array}{l}\text { RTCM method } \\
\text { Step Number }\end{array}$}} & \multirow{2}{*}{$\begin{array}{c}\text { One Step } \\
\text { Step 1 }\end{array}$} & \multicolumn{2}{|c|}{ Adaptive (Two-step) } \\
\hline & & & Step 1 & Step 2 \\
\hline \multicolumn{2}{|c|}{ Target } & ETR to LTR & ETR to STR & STR to LTR \\
\hline \multirow{3}{*}{ AANN outputs } & $\mathrm{P}_{\text {Size }}$ & 60 & 55 & 60 \\
\hline & It $_{\text {Max }}$ & 42 & 45 & 40 \\
\hline & $\mathrm{D}_{\mathrm{R}}$ & 1 & 0 & 0 \\
\hline \multicolumn{2}{|c|}{$\mathrm{T}_{\mathrm{S}}(\mathrm{s})$} & 9.6 & 9.5 & 9.3 \\
\hline \multicolumn{2}{|c|}{$\mathrm{T}_{\mathrm{cc}}^{\mathrm{Max}}(\min )$} & 5 & 5 & 10 \\
\hline \multicolumn{2}{|c|}{ PST 16-17 (Tap position) } & 1 & 7 & 3 \\
\hline \multicolumn{2}{|c|}{ PST 08-09 (Tap position) } & 9 & 10 & 8 \\
\hline \multicolumn{2}{|c|}{$\sum_{\mathrm{L}=1}^{\mathrm{NL}} \Delta \mathrm{P}_{\mathrm{L}}(\mathrm{MW})$} & 255.40 & 0 & 0 \\
\hline \multicolumn{2}{|c|}{$\sum_{\mathrm{g}=1}^{\mathrm{Ng}} \Delta \mathrm{P}_{\mathrm{g}}(\mathrm{MW})$} & 270.85 & 182.97 & 411.45 \\
\hline \multicolumn{2}{|c|}{ Total cost $(\$ /$ minutes $)$} & 34319 & 4913 & 8891 \\
\hline \multicolumn{2}{|c|}{ Total cost in the $\mathrm{T}_{\mathrm{cc}}^{\mathrm{Max}}$ time span (\$) } & 165,875 & \multicolumn{2}{|c|}{111,147} \\
\hline \multicolumn{2}{|c|}{ Congested Lines } & $06-07$ & $06-07$ & 06-07 \\
\hline \multicolumn{2}{|c|}{ Loading before RTCM (\%) } & 137.30 & 137.30 & 117.98 \\
\hline \multicolumn{2}{|c|}{ Loading after RTCM (\%) } & 98.40 & 117.98 & 98.55 \\
\hline
\end{tabular}

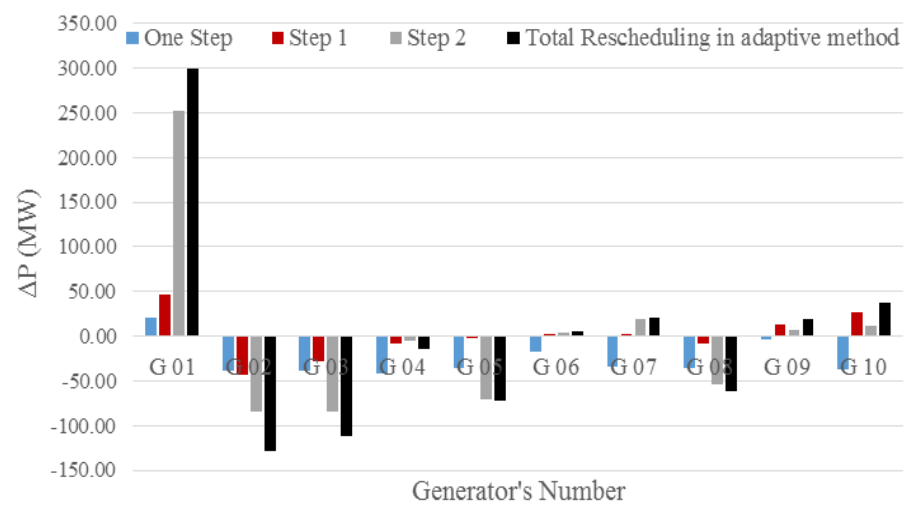

Figure 8.8: Rescheduling values for all solution in scenario 1.

It is because of this fact that the estimated values by the ANN were imported to the PSO problem, and it was successful in finding a feasible solution during the solution time. Furthermore, it is observed that the total RTCM cost for the adaptive method is significantly less than the value for the one-step RTCM method because in this contingency when the adaptive method is used for the RTCM problem, it is not required to use DR ( $D_{R}$ $=0$ ) while it is needed when the one-step RTCM method is applied. Figure 8.8 shows the generators' rescheduling values for solutions of scenario 1. 
Table 8.5: RTCM results in scenario 2.

\begin{tabular}{|c|c|c|c|c|c|c|c|c|c|c|}
\hline \multirow{2}{*}{\multicolumn{2}{|c|}{$\begin{array}{l}\text { RTCM method } \\
\text { Step Number }\end{array}$}} & \multirow{2}{*}{\multicolumn{3}{|c|}{$\begin{array}{c}\text { One Step } \\
\text { Step 1 }\end{array}$}} & \multicolumn{6}{|c|}{ Adaptive } \\
\hline & & & & & \multirow{2}{*}{\multicolumn{3}{|c|}{$\begin{array}{c}\text { Step } 1 \\
\text { ETR to STR }\end{array}$}} & \multicolumn{3}{|c|}{ Step 2} \\
\hline \multicolumn{2}{|c|}{ Target } & \multicolumn{3}{|c|}{ ETR to LTR } & & & & \multicolumn{3}{|c|}{ STR to LTR } \\
\hline \multirow{3}{*}{ AANN outputs } & $\mathrm{P}_{\text {Size }}$ & \multicolumn{3}{|c|}{58} & \multicolumn{3}{|c|}{55} & \multicolumn{3}{|c|}{60} \\
\hline & It $_{\text {Max }}$ & \multicolumn{3}{|c|}{42} & \multicolumn{3}{|c|}{45} & & 40 & \\
\hline & $\mathrm{D}_{\mathrm{R}}$ & \multicolumn{3}{|c|}{1} & \multicolumn{3}{|c|}{0} & \multicolumn{3}{|c|}{0} \\
\hline \multicolumn{2}{|c|}{$\mathrm{T}_{\mathrm{s}}(\mathrm{s})$} & \multicolumn{3}{|c|}{9.6} & \multicolumn{3}{|c|}{9.6} & \multicolumn{3}{|c|}{9.3} \\
\hline \multicolumn{2}{|c|}{$\mathrm{T}_{\mathrm{cc}}^{\mathrm{Max}}(\min )$} & \multicolumn{3}{|c|}{5} & \multicolumn{3}{|c|}{5} & \multicolumn{3}{|c|}{10} \\
\hline \multicolumn{2}{|c|}{ PST 16-17 (Tap position) } & \multicolumn{3}{|c|}{-1} & \multicolumn{3}{|c|}{-5} & \multicolumn{3}{|c|}{-10} \\
\hline \multicolumn{2}{|c|}{ PST 08-09 (Tap position) } & \multicolumn{3}{|c|}{-10} & \multicolumn{3}{|c|}{-7} & \multicolumn{3}{|c|}{-10} \\
\hline \multicolumn{2}{|c|}{$\sum_{\mathrm{L}=1}^{\mathrm{NL}} \Delta \mathrm{P}_{\mathrm{L}}(\mathrm{MW})$} & \multicolumn{3}{|c|}{501.62} & & 0 & & & 0 & \\
\hline$\sum_{\mathrm{g}=1}^{\mathrm{Ng}} \Delta \mathrm{P}_{\mathrm{g}}$ & & & 193.58 & & & 0 & & & 177.4 & \\
\hline Total cost $(\$ /$ & & & 55,831 & & & 0 & & & 2316.70 & \\
\hline Total cost in the $\mathrm{T}_{\mathrm{cc}}^{\mathrm{M}}$ & $\operatorname{span}(\$)$ & & $269,84 \mathrm{C}$ & & & & & & & \\
\hline Congested & & 04-05 & 04-14 & 06-11 & 04-05 & $04-14$ & 06-11 & 04-05 & 04-14 & 06-11 \\
\hline Loading before & $(\%)$ & 123 & 106 & 113 & 123 & 106 & 113 & 109 & 103 & 113 \\
\hline Loading after $\mathrm{R}$ & $(\%)$ & 99 & 95 & 105 & 109 & 103 & 113 & 93 & 94 & 98 \\
\hline
\end{tabular}

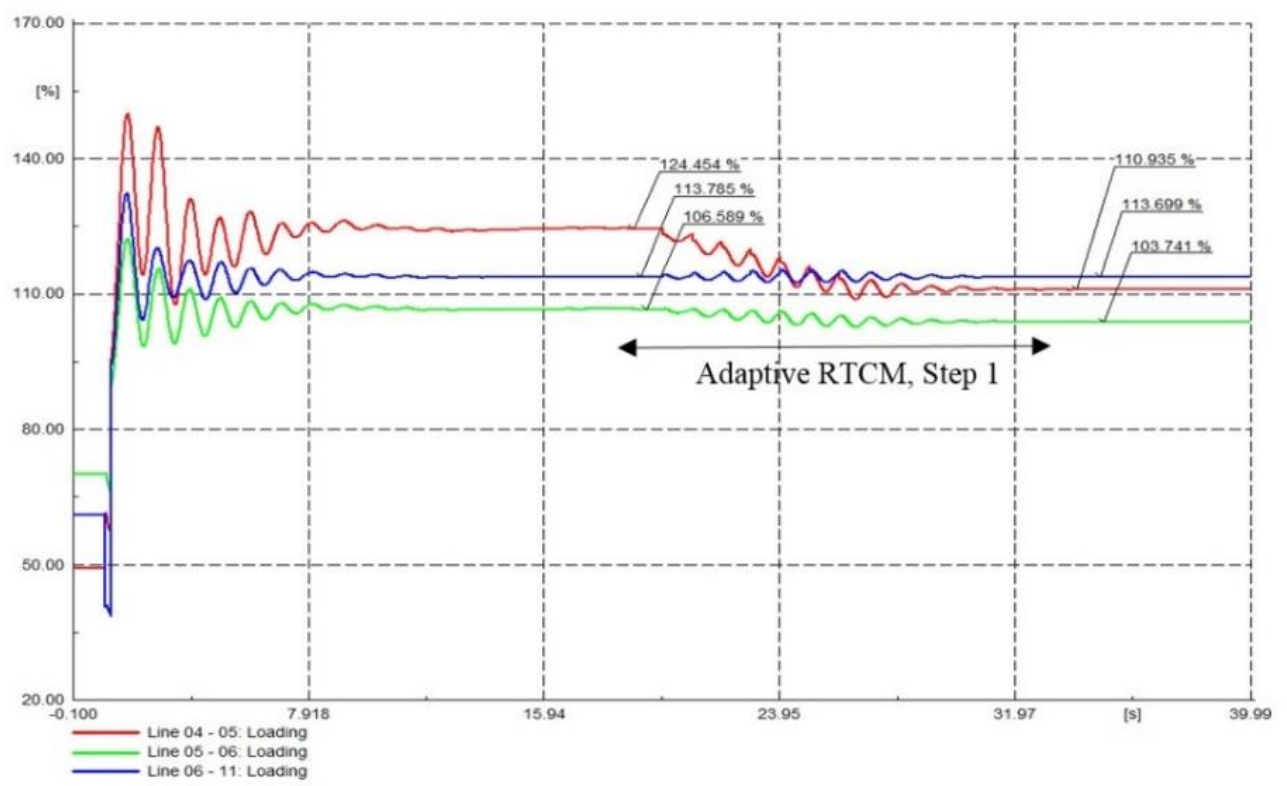

Figure 8.9: Congested lines' loading in scenario 2.

\subsubsection{Scenario 2}

For the second scenario, the N-1 contingency for the line 13-14 is modeled. By modeling a three-phase short circuit (at $\mathrm{t}=1 \mathrm{sec}$.) and protection relays tripping (at $\mathrm{t}=1.2$ sec.), three transmission lines will be congested as shown in Figure 8.9. Since the most congested line (Line 04-05) is in its ETR region (118\% <loading < 147\%), the adaptive RTCM method tries to go from the ETR to STR level at the first step. This means that, 
instead of clearing the congestion in one step and trying to mitigate the $47 \%$ of congestion, we divide it into two step, at first we consider mitigation from $47 \%$ to $18 \%$ within emergency time, afterward the RTCM will mitigate the remained $18 \%$ overload during the short time with more relaxed constraints, which will result in lower congestion management cost.

Numerical results for this scenario have been summarized in Table 8.5. From these results, by using the one-step RTCM method, although all PSTs, generates, and loads are participating in the RTCM problem, the final solution is not feasible because the line 0611 still has $5 \%$ overload in this case. However, it is obviously clear that the adaptive method is highly successful in managing congestion optimally. At the first step, the RTCM cost is zero because the algorithm only needs PSTs operation to move from the ETR to the LTR level without any rescheduling and DR (Figure 8.9). Furthermore, in the second step, only generation rescheduling is adequate for the RTCM. This scenario shows both the technical and economic advantages of the developed adaptive RTCM algorithm. In other words, having more available clearing time and releasing optimization constraints will result in a more reliable, cost-effective and optimal solution for the RTCM problem.

\subsection{Summary}

In this chapter, we developed an adaptive real-time congestion management algorithm considering the adaptive thermal rating of transmission lines, which uses the thermal adaption of the conductors in two steps during the congestion management process. Dividing the congestion removal progress into the two phases, incorporating the FACTS devices capability and DR in the optimization problem, made it possible to find feasible

and considerably cost-effective solutions for the RTCM problem. Furthermore, a hybrid 
real-time optimization framework was developed based on the AANN and the modified PSO algorithm, which is considered as a highly accurate and robust optimization tool for the RTCM problem. The developed algorithm has a comprehensive concept of the RTCM ancillary service in a deregulated environment, where all market players can participate in it. Numerical results illustrated that the developed algorithm is cost-effective, highly secure and reliable in critical cases when the one-step RTCM method fails to manage the congestion properly. 


\section{Chapter 9 Critical Communications in Modern Power Systems}

In this chapter, a data-centric communication framework is developed for MRGM messages over the WAN for effective SS2SS and SS2CC communications. In this structure, the IEC 61850 GOOSE message is transmitted over the WAN using the DDS as

a fast, reliable, and secure data-centric communication middleware. The main feature of this framework is its multicast capability, where several authorized subscribers can receive a published message simultaneously. This can significantly improve the system monitoring and control of the protection systems in modern smart grids, where intelligent schemes can be applied. The effectiveness of the developed platform, in terms of total end-to-end delay between participants, is evaluated through experimental results obtained from the actual hardware-based test setup developed at the FIU smart grid testbed. The results demonstrate that the latency between sending and receiving a GOOSE message among participants is within its maximum time span defined by the IEC 61850-90-5 working group for communications over the WAN.

\subsection{Introduction}

The contribution of this research is to address the above-mentioned weaknesses for fast, multicast, and reliable IEC 61850 R-GOOSE communications over WAN in modern power systems. Therefore, the state-of-the-art of this work could be summarized as follow:

- Introducing a DDS-based communication framework for multicast R-GOOSE messages;

- Describing unique features of this framework in terms of scalability, reliability, and multicasting;

- Addressing the network traffic issues and minimize the end-to-end time delay; 
- Verifying the developed approach through an experimental setup by measuring the real-time end-to-end delay of R-GOOSE message communication over the network layer.

\subsection{The Proposed MRGM Framework}

Figure 9.1 shows the proposed DDS-based communication framework for MRGM over the WAN. It also contains two algorisms for GOOSE message transition from the first substation (Substation A) to the second one (Substation B) which are assumed to be in two different LAN. As can be seen in this figure, the DDS is selected as a data-centric communication middleware for GOOSE data exchange between two participants in two substations. The IEC 61850 defines a RTPS protocol for GOOSE data exchange within the wall of a substation. In this protocol, the GOOSE message is published by the GOOSE publisher to the local network with specific format including SqNum, StNUm, GOOSE dataField, and an APPID (It is the ID of the published message which is checked by subscribers).

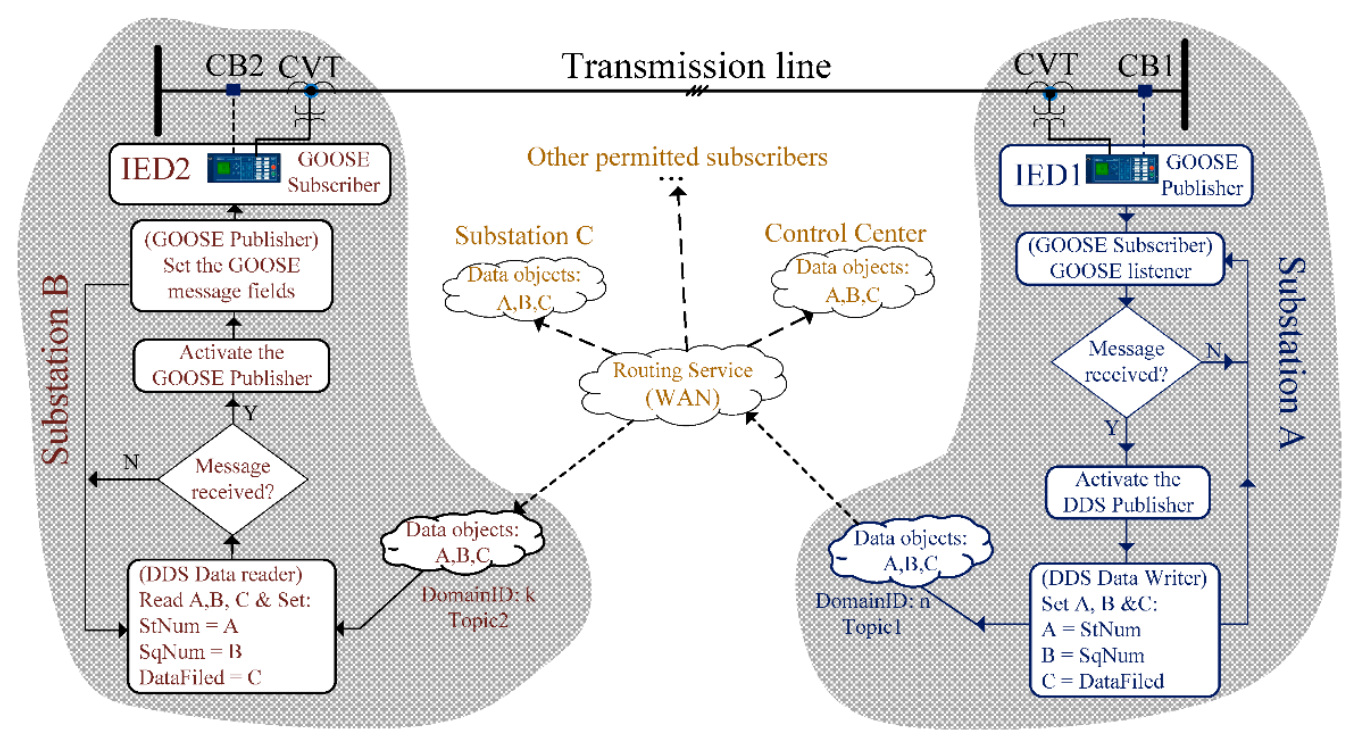

Figure 9.1: Proposed Multicast Routable GOOSE Messages Framework. 


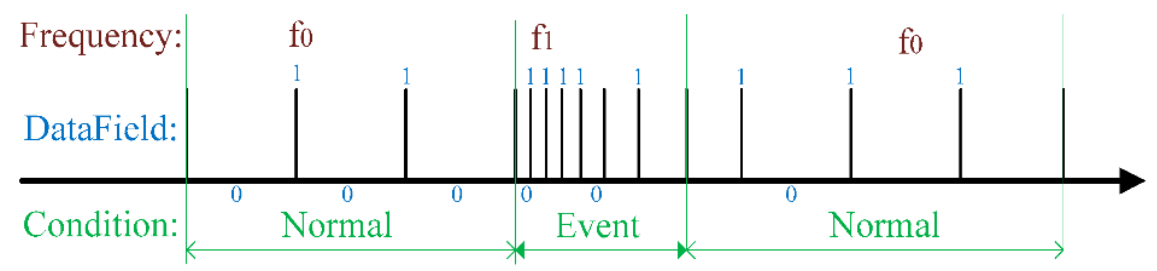

Figure 9.2: The mechanism of the GOOSE repetition.

Once the message is published, subscribers with the same APPID instantly subscribe to the message and praise the message to take an appropriate action. The rate of sending GOOSE messages varies due to the system condition. In normal operating mode, where there is no need for protection relays' actions, GOOSE messages are sent periodically to test the communication system. It usually happens every one second.

The high-frequency cascading messages are sent due to protection action requirement because of fault detection in the system or control commands. In this situation, as shown in Figure 9.2, a train of GOOSE messages are sent to the network starting with a very high frequency and continue to lower frequency rates to make sure that the messages are received by appropriate subscribers to cover the possibility of missing samples. The standard has defined a $4 \mathrm{~ms}$ time span for GOOSE message delivery within the data link (Layer 2 of the OSI model). However, the published GOOSE messages could be needed to be subscribed by other IEDs in other substations or by the control/monitoring center. For this purpose, GOOSE messages should be transferred from the data layer (Layer 2 of OSI model) to the network layer (Layer 3 of OSI model) and routed to their destinations in the system over the internet network. The standard has defined a $10 \mathrm{~ms}$ time span for routable GOOSE (R-GOOSE) messages.

As mentioned before, specific network settings are required to address this fast delivery over the WAN, therefore, in this dissertation we propose to deploy the DDS standard for 
R-GOOSE messages as it covers all the required communication needs. Furthermore, it provides the multicast feature of R-GOOSE messages at the network layer, the same as GOOSE messages at the data layer. This means that several subscribers could receive a published GOOSE message simultaneously and take proper actions immediately. This feature is very helpful in advanced protection systems. In [195], authors have presented an advanced protection scheme for a modern power system, which are experiencing a high level of fault current beyond the breaking capacity of circuit breakers. The developed advanced protection scheme is mainly based on R-GOOSE messages over the WAN and assumes that a multicast R-GOOSE message framework could deliver the messages to its destination in less than $10 \mathrm{~ms}$. This research addresses this framework and demonstrating its capabilities through experimental results. The developed MRGM algorithm includes three main steps starting with GOOSE publisher in the first network, routing the message over the WAN, and subscribing it within the second network.

\subsubsection{Conversion: GOOSE Data Model to DDS Data Object}

As shown in the right side of Figure 9.2 (Substation A), the R-GOOSE communication is begun like the conventional GOOSE publishing in data layer. Since GOOSE data model has been defined in the IED platform, to change and add or modify the feature of message we need to develop agents with access to the same network, where GOOSE message is published. This agent is equipped with a GOOSE subscriber with the same APPID of the GOOSE message that we need to send it outside the wall of this substation. Once the GOOSE message is subscribed by this agent, the algorithm is activated by praising the received message, extracting its parameters, and activating a DDS publisher. As mentioned before, a GOOSE data model contains important parameters, i.e., StNum, SqNum, and 
DataField. For broadcasting these parameters by a DDS publisher, a proper IDL file should be defined by suitable data format for each parameter. The StNum and SqNUm contain integer values while DataField has the Boolean type. Therefore, these data types are defined on a DDS topic (e.g., Topic1) within a DDS domain (e.g., Domain n) by three variables (A, B, and C). The activated DDS publisher writes the received parameters' updates on this topic.

\subsubsection{Message Encapsulating and Routing Service}

To encapsulate this message and route it over the WAN, the DDS routing service is activated and simply routes this message over the network router and delivers it to a predefined topic within a DDS domain in another network, where DDS subscribes are waiting to read data. This is possible just by defining the IP address of both DDS publisher and subscriber through the DDS routing service profile.

To meet the maximum end-to-end delay for R-GOOSE communication, specific network settings are needed. The DDS QoS profile contains control mechanism and policies for data transfer over the network. For example, it could control availability of data, life span, latency budget, etc. The message delivery time is defined by the latency budget, i.e., the index which shows the maximum allowed time span for the message delivery. To control this latency, message priority indices could be attached to timesensitive and critical messages, therefore, the network switches transfer the received messages based on the priority policies, regardless of data queue. This means that in limited bandwidth networks with high message deliver latencies, which usually is due to the first come first serve policy, defining the message priority profiles could significantly enhance the critical message communications over the internet network. The DDS supports three 
priority policies including round Ronin (RR), earliest deadline first (EDF), and high priority first (HPF). The RR is known as the simplest priority scheduling scheme which processes the messages in the order they have been received. It prioritizes the messages considering their latency budget scheduled in EDF policy, where the messages with less remaining time span are processed first. It is worth mentioning that the EDF is the default policy scheduled by the DDS. The combination of the RR and EDF could be scheduled by HPF policy, where the messages with the same latency budget are processed by the RR policy.

\subsubsection{Conversion: DDS Data Object to GOOSE Data Model}

By delivering the message to its destination in the Substation B' network, a predefined topic (e.g., Topic2) within a domain (e.g., Domain k), a DDS subscriber deploys its data reader to receive data, as shown in the Figure 9.2, Substation B' algorithm. Afterward, these data are tuned within a GOOSE publisher and published to the local network over the data link layer. Finally, actual IEDs with the same APPID subscribe the message, verify, and react to it. The same algorithm is run with all other substations or monitoring centers which are supposed to receive data from this GOOSE publisher. The multicast feature and simultaneous subscribing of the R-GOOSE message enable us to implement real-time, reliable, and advanced protection, control, or remedial actions in modern smart power systems [195].

\subsection{Test Framework}

To evaluate the performance of this framework in terms of data delivery and delay time, we developed a test framework, as shown in Figure 9.3. The purpose is to measure the latency of the algorithm for both GOOSE publishing and subscribing in the data link layer 
and the DDS time delay over the network layer. This framework contains four Linux-based embedded agents shown by numbers (1) to (4).

Agent (1), receives the analog input signal, converts it to digital mode (0/1), sets the GOOSE DataField according to this value, and publishes it to the network by the APPID \# 1000.

Agent (2) has an active GOOSE subscriber with the same APPID (\#1000) and subscribes to this message. The GOOSE DataField along with StNum and SqNum are extracted and are used by DDS data writer to publish these data over the DDS topic.

In Agent (3), the DDS message is subscribed and being published by the GOOSE publisher with another APPID (\# 2000).

Agent (4) receives this message, converts its digital DataField value to an analog signal, and delivers it to output ports of this agent, where this signal could be compared with input to determine the time delay due to communication platform.

\subsubsection{Hardware Setup}

According to Figure 9.3, we developed a hardware-based experimental setup at FIU smart grid testbed, shown in Figure 9.4. In this setup, we used four Beaglebone Blacks as defined agents for this communication framework (each one has an AM335x Arm® Cortex-A8 with $1 \mathrm{GHz}$ processor and $512 \mathrm{MB}$ RAM), a function generator to generate the input signal with 3.2 volte amplitude and frequency of $40 \mathrm{~Hz}$, and an oscilloscope to monitor and compare the input and output signals. A PC was deployed to monitor the RTI admin console, where we can monitor the DDS system participants including the publishers and subscribers, DDS domains, and topics. 


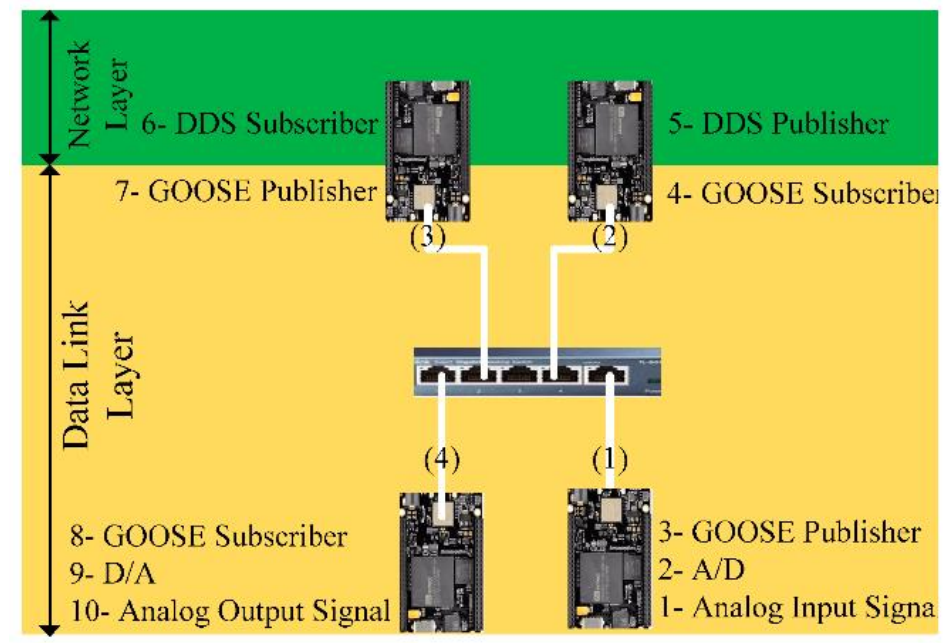

Figure 9.3: The test framework configuration.

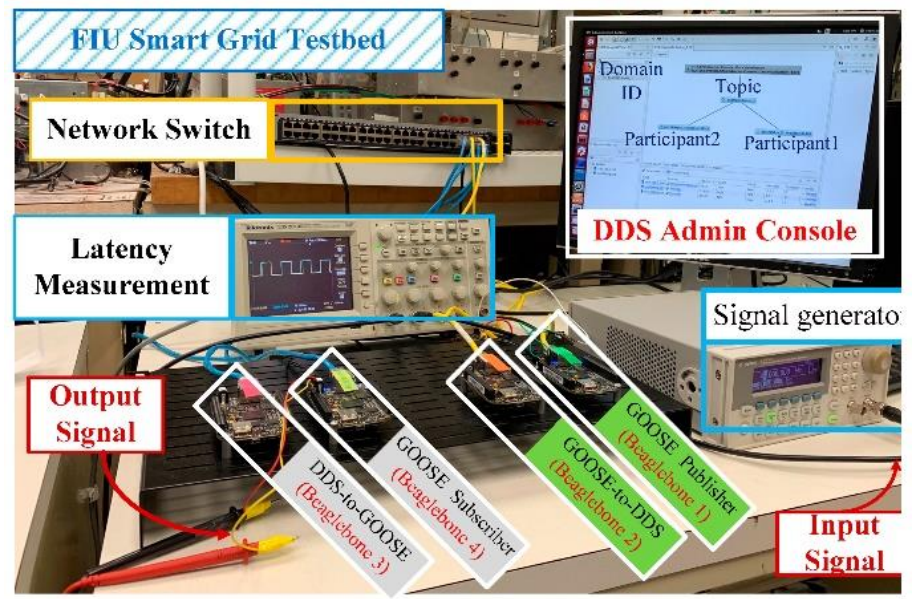

Figure 9.4: Experimental setup at FIU smart grid testbed.

\subsection{Experimental Results}

The experimental results are presented here and described in detail

\subsubsection{GOOSE Publish-Subscribe over Layer Two: Case1}

This experiment is executed using two agents, as shown in Figure 9.5. To evaluate the latency of the GOOSE delivery, we needed to examine a large number of samples (around $1 \mathrm{k}$ ) because of various time delays between input and output signals. It is because of this fact that the processors deal with each sample differently, therefore, we had calculated the average latency of GOOSE communication over the data link layer. 


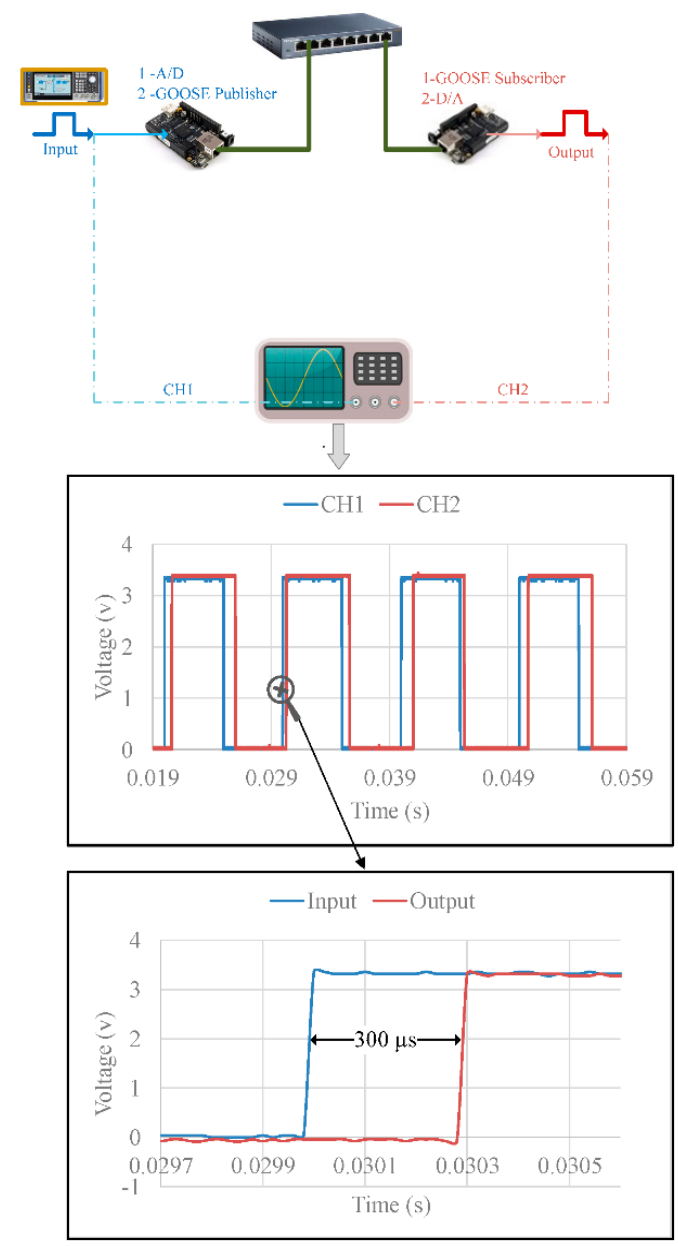

Figure 9.5: Results from Case 1.

Table 9.1: Measurement results.

\begin{tabular}{ccccc}
\hline \multirow{2}{*}{ Study Case } & \multicolumn{4}{c}{ Latency $(\mu \mathrm{s})$} \\
\cline { 2 - 5 } & Min. & Max. & Ave. & S.D. \\
\hline 1 & 300 & 1300 & 500 & 50 \\
\hline 2 & 980 & 2700 & 2100 & 160 \\
\hline
\end{tabular}

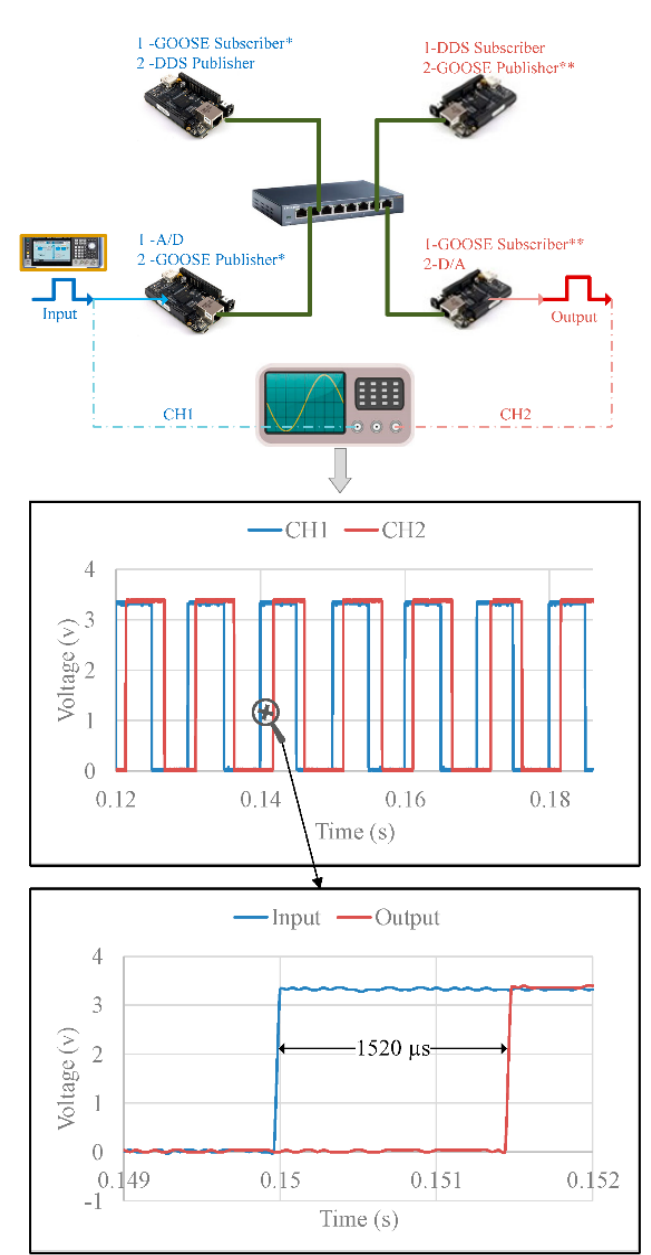

Figure 9.6: Results from Case 2.

Table 9.2: Average latency.

\begin{tabular}{cc}
\hline Section & Latency $(\boldsymbol{\mu s})$ \\
\hline$T_{G}$ & 500 \\
\hline$T_{D}$ & 1100 \\
\hline$T_{R}$ & 70 \\
\hline$T_{N}$ & 7830 \\
\hline
\end{tabular}

The minimum and maximum latency recorded in our measurements were around 300 and $1300 \mu \mathrm{s}$, respectively, and the average value was around $0.5 \mathrm{~ms}\left(\mathrm{~T}_{\mathrm{G}}=500 \mu \mathrm{s}\right)$ with a standard deviation (S.D.) of $50 \mu$ s. The low value of the standard deviation indicates that the average value is a good index to demonstrate the time latency of GOOSE communication at data link layer. 


\subsubsection{DDS Publish-Subscribe over the Network Layer: Case2}

To measure the DDS delay, we had to measure the total end-to-end delay (Tt) by the Developed test framework, as shown in Figure 9.6. As mentioned before, this communication occurred by two GOOSE publish-subscribe actions plus a DDS data exchange. Therefore, by measuring the total end-to-end delay, the DDS delay time (TD) is calculated by (9-1).

$$
T_{D}=T_{t}-\left(2 \times T_{G}\right)
$$

The average value of the total end-to-end delay $\left(T_{t}\right)$ was obtained by recording a large number of samples (around 1k) as we did in Case 1. The latency of this case is changing between 1000 and $2700 \mu \mathrm{s}$. The average and standard deviation are 2100 and $160 \mu \mathrm{s}$, respectively. As a result, and as shown by (2), the DDS delay time is $1100 \mu$ s. Table 9.1 summarizes the results for these two study cases.

$$
T_{D}=2100-(2 \times 500)=1100 \mu \mathrm{s}
$$

The test framework does not consider the latency of the DDS routing service and network traffic. However, experimental results report an average of around $70 \mu$ s for the DDS routing service action (TR). Hence, the average latency due to network traffic (TN) should not be more than $7830 \mu$ s, as calculated by (9-3).

$$
T_{N}=T_{\text {Max }}-\left(2 \times T_{G}+T_{D}+T_{R}\right)
$$

In this equation, $\mathrm{T}_{\mathrm{Max}}$ is the maximum allowed time delay for R-GOOSE messages, which is $10 \mathrm{~ms}$, based on IEC 61850 standard. The average time delay for each section of this communication framework is reported in Table 9.2. These calculations mean that the developed framework is fast enough and only needs around $2170 \mu$ s to encapsulate a GOOSE message and transfer it by the DDS and give us $7830 \mu$ s for communication over 
the WAN, which is feasible by defining a tunnel between two communicating networks along with a proper message priority policy. Generally, the network latency is highly dependent on the size of the message, network bandwidth, and performance (unicast/multicast features). As reported in [196], for a 32 bytes message size with the rate of $1 \mathrm{k}$ message per second, the best effort of the DDS QoS profile resulted in $269 \mu$ s latency for the communication system. It means that using a proper QoS profile and tunneling technique, the R-GOOSE communication would be feasible over the maximum time span (7830 $\mu \mathrm{s})$ available when the Developed framework is utilized.

\subsection{Summary}

In this chapter, a multicast communication framework was developed for R-GOOSE messages over the WAN. The skeleton of this framework is based on a data-centric communication approach to address the technical requirements for time sensitive and critical communications in smart power systems. This approach encapsulates the GOOSE data model into the DDS data object and routes it over the network to its final destinations. The effectiveness of the Developed framework was validated by the experimental results by measuring the latency of this framework, showing that this approach only took around $20 \%$ of the maximum defined time for IEC 61850 routable communication and give us around $8 \mathrm{~ms}$ to manage the network traffic. Furthermore, the best effort of the QoS profile along with the proper message priority policy could guarantee the message delivery within its defined time span. 


\section{Chapter 10 Security of Critical Communications in Power Systems}

In Chapter 9, a DDS-based communication framework was developed for MRGM over the WAN for effective SS2SS and SS2CC communications. However, we did not implement any security algorithm on the MRGM framework to protect it against possible cyberattacks, which is a critical issue for such an important communication in power systems. In this chapter, the security of the MRGM framework is studied to address the proper security algorithm for this critical communication within its limited time span. At first, the DDS security measure is implemented for user authentication and message encryption/decryption. Afterward, a new security algorithm, which is called the SSHA, is introduced to ensure the data integrity and security for routable communications over the WAN in smart power systems. This algorithm could be implemented for SS2SS and SS2CC communications when a fast and secure method is required to transfer critical messages over the network, such as GOOSE messages. The developed SSHA security algorithm not only guarantees data integrity but also provides a reliable security algorithm based on the concept of sequence hopping. The experimental hardware setup is developed to implement and evaluate the developed algorithm to measure the end-to-end latency, evaluate possible cyber-attacks, and monitor the communication system. The experimental results demonstrate that the developed framework is fast and secure enough to meet the predefined ten milliseconds time delay for IEC 61850 routable communications and is robust sufficient against cyber-attack scenarios.

Figure 10.1 shows two substations in the power system and a transmission line which connects them physically. As can be seen, protective relays within each substation can communicate using GOOSE and SV protocols over the data layer of the internal substation 


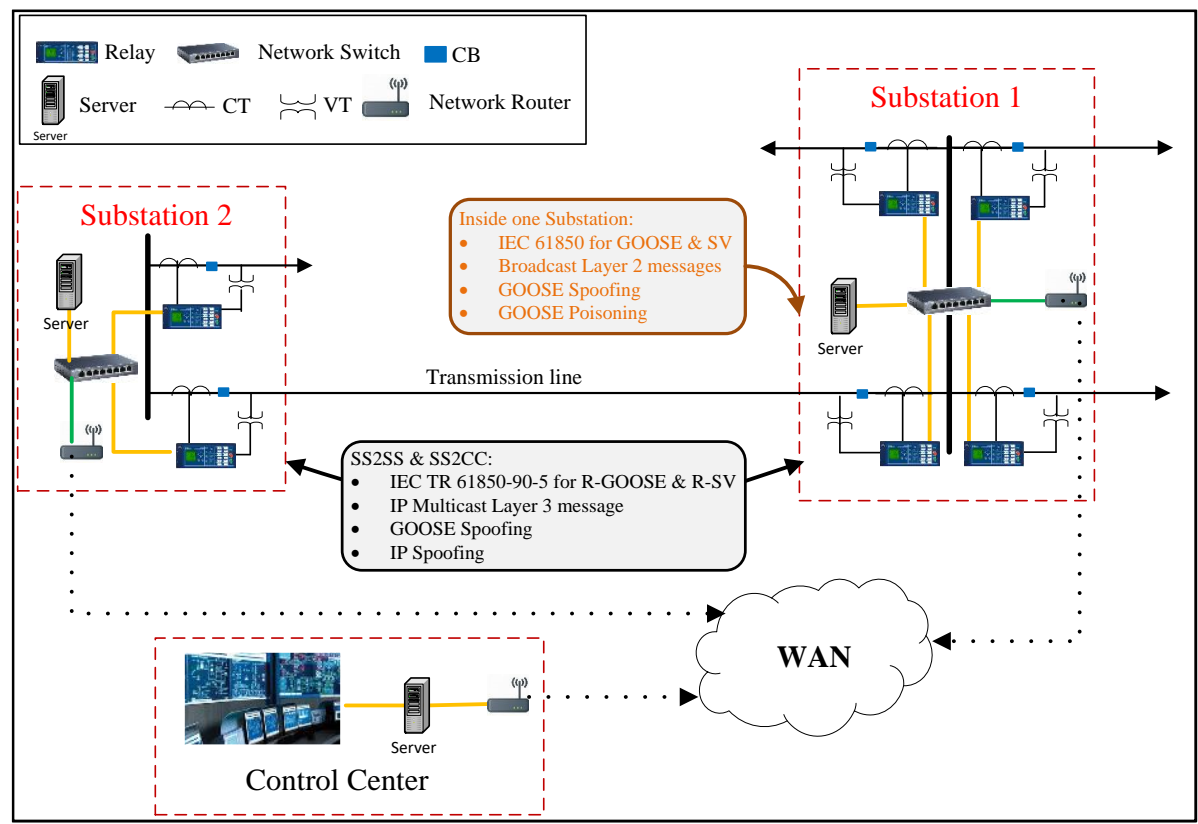

Figure 10.1: Critical communications in power systems and possible threats

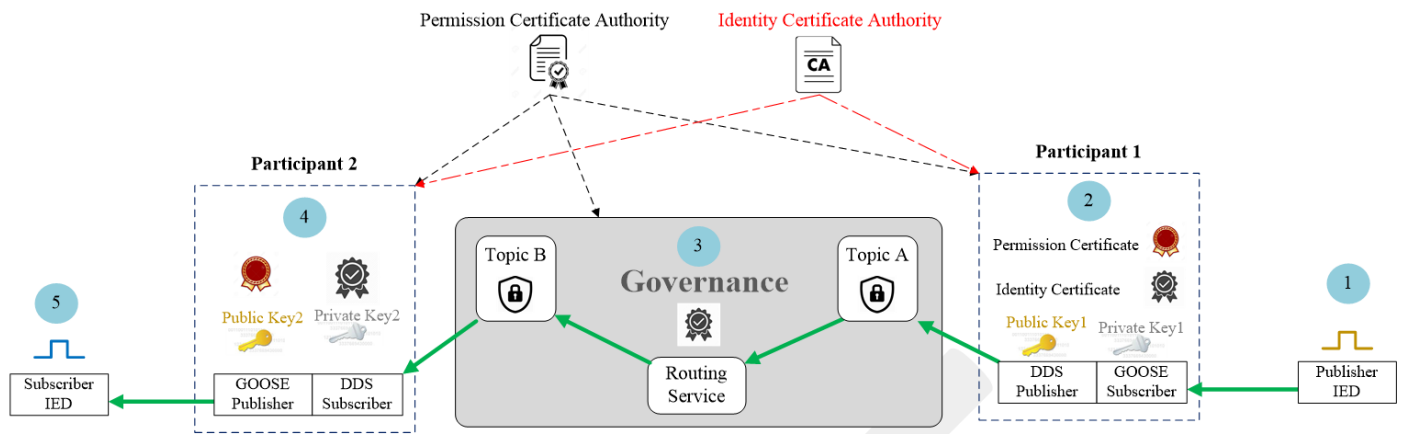

Figure 10.2: Secure DDS-Based MRGM framework

network. However, for SS2SS and SS2CC, relays need to communicate over the network layer, where other threats such as IP spoofing are possible. In other words, routable communications will introduce a new surface of attack including ARP spoofing and other man-in-the-middle attacks.

\subsection{DDS Security Mechanism for the MRGM Framework}

The developed MRGM framework maps the GOOSE data model to DDS data Objects. Ultimately, a specific DDS Global Data Space (domain) is being secured and, within that domain, the ability to read or write information is controlled. 
Generally, as mentioned in Chapter 5, DDS has its own security algorithm to authenticate the users, encrypt messages and check data integrity in the network. It contains key management by a shared CA and permission access by permission CA, as shown in Figure 10.2. The CA checks the participants' authentication while the permission CA is used to control participants' access to different topics. For example, DDS participant1 just needs to write a DDS message on the Topic A. Hence, the permission access for this participant only allows it to write on this topic and denies its access to read data. On the other side, Participant2 gets access to read from Topic B and DDS routing service is permitted to read from Topic A and write to Topic B. Based on these permissions, each participant requests the permission CA to sign its permission file and utilizes it once communicate with other participants.

Using DDS, users can customize the behavior and technologies for authentication, access control, encryption, digital signing, logging, and data tagging. To provide secure access to the DDS Global Data Space, applications that use DDS (The Domain Participant) must first be connected to the same network infrastructure that other participants are connected to it. Afterward, the authentication is required to ensure that the right participant originated the message. So that the identity of the application can be established by a HMAC or digital signature. The HMAC creates a message authentication code using a secret key that is shared with the intended recipients while a digital signature is based on public-key cryptography.

It is worth mentioning that in many situations, users prefer to deploy the HMACs because its performance is more efficient and much faster than computing/verifying digital signatures. Once authentication is obtained, the next step is to enforce access control 
decisions that determine whether the application is allowed to perform specific actions. Examples of these actions include joining a DDS Domain, defining a new Topic, reading or writing to/from a DDS Topic, and even reading or writing specific Topic instances. Enforcement of access control shall be supported by cryptographic techniques so that information confidentiality and integrity can be maintained, which in turn requires an infrastructure to manage and distribute the necessary cryptographic keys.

\subsection{Test Framework}

To verify the effectiveness of the developed framework, additional latency due to the DDS security algorithm should be measured. For this aim, the configuration of the test system is shown in Figure 10.3 including required network connections and hardware, and Figure 10.4 shows the developed hardware setup including the input square pulse signal (generated by a function generator with a frequency of $40 \mathrm{~Hz}$ and amplitude of 3.2 volts); An Oscilloscope to monitor this signal in the channel $1(\mathrm{CH} 1)$, Beaglebone1 (Beaglebone Black with an AM335x Arm ${ }^{\circledR}$ Cortex-A8 with $1 \mathrm{GHz}$ processor and $512 \mathrm{MB}$ RAM) to convert this analog signal to digital (True and False) and publish a GOOSE message to the network; PC1 (Core i7 CPU @ $3.2 \mathrm{GHz}$ with 8 GB of RAM) to listen to this message and capsulizes its data field content over a DDS publisher' data object, sign and publish it to the predefined topic in the virtual data space; PC2 to read this data from the topic, decrypt the message, get the content and publish it as a GOOSE message to the network; BeagleBone2 to subscribe this message and convert it to the analog output and monitor it by second channel $(\mathrm{CH} 2)$ of the Oscilloscope to compare with the input signal and measure the end-to-end delay for this GOOSE transmission between two substations. 


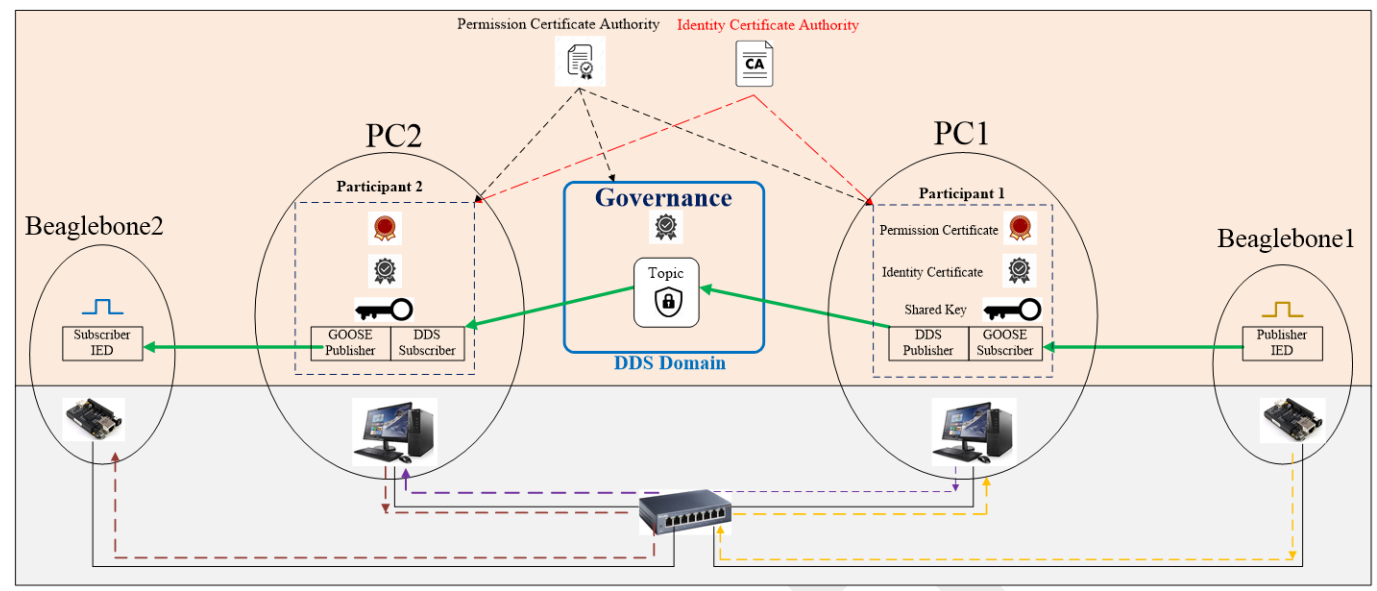

Figure 10.3: The test framework configuration and network connections.

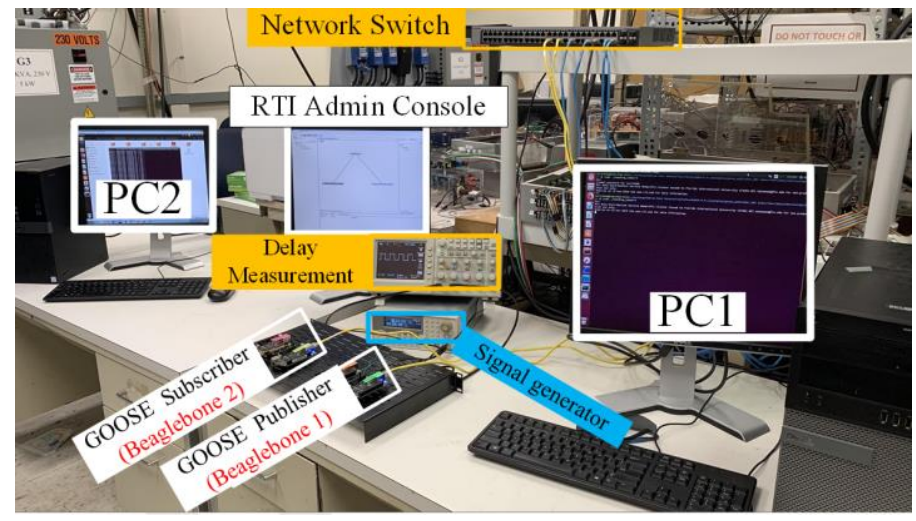

Figure 10.4: Experimental setup at FIU testbed.

\subsection{Attack model}

As mentioned in Figure 10.2, for communication over the IP layer man in the middle attack, IP spoofing, unauthorized publishing, and data sniffing are possible. Understanding the major threats for DDS is necessary to recognize how the decisions are made by the security mechanism. Generally, there are three potential threats for DDS [197]:

1. Unauthorized publication/subscription

2. Tampering and replay

3. Unauthorized access to data by Infrastructure Services.

These attacks occur when the DP connects to the same network where other participants 
are communicating. In this situation, it can subscribe to the messages that are not sent to another DP or publish messages with any data content to a topic that other agents are publishing/subscribing to it. This is a kind of tampering and replay by spoofing the system and injecting bad data. Therefore, the security model needs to support the concept of having a participant who is allowed to receive, process, and relay RTPS messages.

\subsection{Experimental results}

In Chapter 9, we calculated the average latency of GOOSE publishing and subscribing over layer 2 and time delay for DDS communication over layer 3. In this section, we want to measure the latency of the DDS security algorithm and lunch a cyber-attack on this system to evaluate the robustness of this system.

\subsubsection{The latency of the security algorithm}

Figure 10.5a demonstrates the structure of DDS domain for this case study, where participants need their signed certificated authorities along with their public and private keys to communication with each other. Figure $10.5 \mathrm{~b}$ indicates the recorded input and output signals which are used to calculate the maximum, minimum and the average values for this case study. The measurements show that the minimum and maximum latencies are 1150,3130 us, respectively. The average time delay is $2236 \mu$ s. It means that the latency of the security algorithm $\left(T_{S}\right)$ is around $136 \mu$ s as calculated by (10-1).

$$
T_{S}=2236-\left(2 \times T_{G}+T_{D}\right)=2236-(2 \times 500+1100)=136 \mu s
$$

This amount of delay shows that DDS security algorithm is very fast, and its latency is acceptable for this application. 


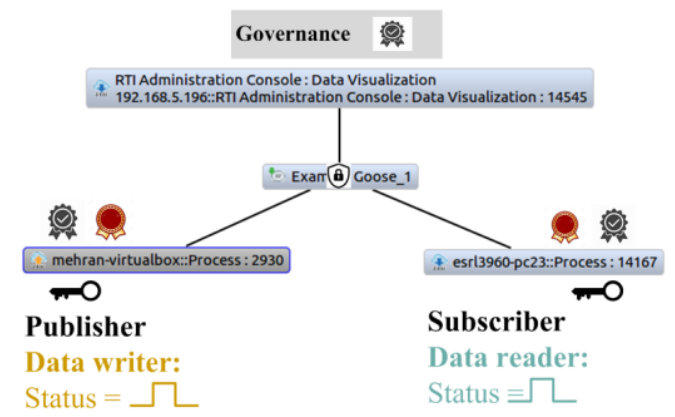

(a)

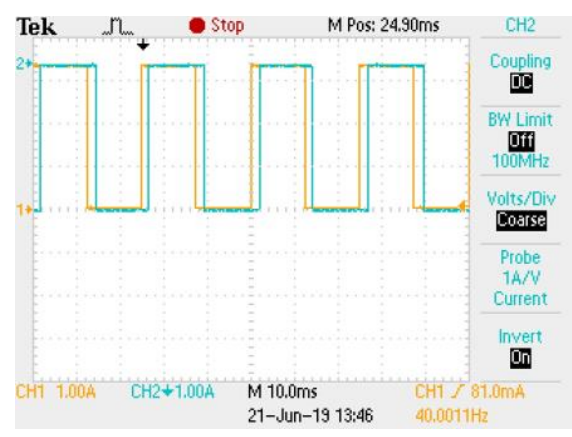

(b)

Figure 10.5: Latency measurement for the MRGM framework with Secure-DDS

\subsubsection{Cyber-attack on the DDS-Based MRGM framework}

The MRGM framework is based on data exchange over the DDS topic in a DDS domain. Therefore, an attacker can monitor the system, detect the DDS domain, topics, and data objects to lunch a successful attack on this system. In this section, we evaluate the robustness of the developed MRGM framework against the false data injection cyberattack for the system without security algorithm and the system which deploys the DDS security mechanism.

\subsubsection{False Data Injection Attack on Non-Secure DDS Topic}

As mentioned before, DDS spoofing and bad data injection are possible cyber threats for the developed R-GOOSE message framework. An attacker can run the RTI admin console in any network which DDS participants belong to and detect the topic name, variable name, as well as the type of the variable (e.g., Boolean, double, integer, etc.). Afterward, the attacker can create a DDS publisher in the same domain ID with an appropriate DLL file, including the same topic and variable name and other characteristics such as data type.

By executing this DDS publisher, the attacker can inject bad data into the system, as shown in Figure 10.6a. In this figure, the actual publisher is sending pulse data (True and False) with a rate of $40 \mathrm{Msg} / \mathrm{s}$. 


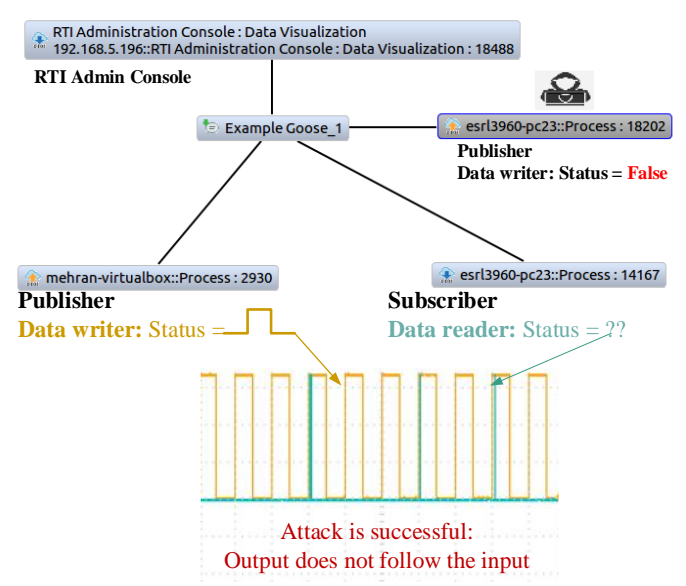

Output does not follow the input

a: Non-secure DDS
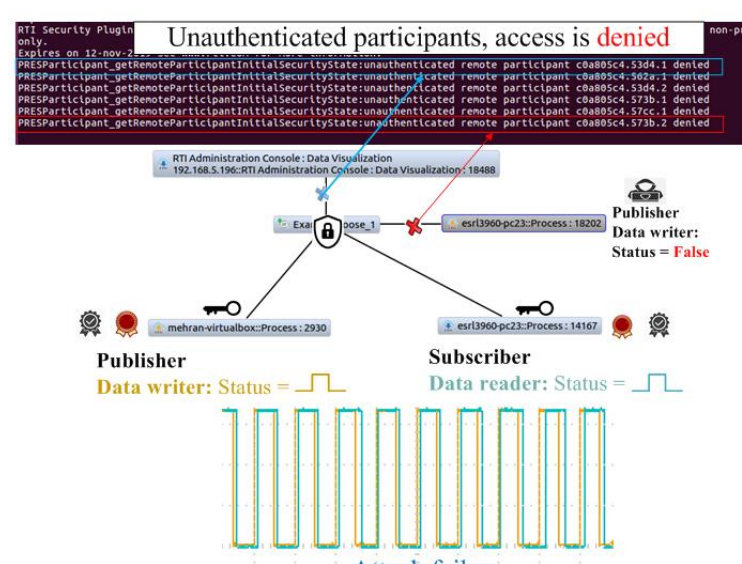

Attack fails:

Output just follows the input

$\mathrm{b}$ : Secure DDS

Figure 10.6: Bad data injection attack on the MRGM framework

The attacker is spoofing the admin console and creating a publisher who sends just false data to the topic, which is named Example GOOSE_1. The result of this attack is also demonstrated in this figure, where the subscriber does not follow the actual publisher's signal. It is because of this fact that it is subscribing to all messages published by a real publisher and the attacker. Therefore, the output is zero for most of the subscribed messages.

\subsubsection{False Data Injection Attack on the Secure DDS Topic}

In this case study, the robustness of the developed secure R-GOOSE framework against a cyber-attack is evaluated and confirmed. For this purpose, an attacker tries to detect the domain participants, DDS topic, and data model to create a fake publisher with malicious data. Due to the DDS security mechanism, just authorized participants can get access to domain data, including the topic name and data model. As a result, when the attacker runs the admin console to monitor and detect the system parameters, the authentication mechanism denies its request, as shown in Figure 10.6b. In fact, by running the RTI admin console, the defined secure topic will not be shown on the domain, and the DDS interface 
shows the alarm of unauthorized participants. However, if by any method the attacker could get access to the name and data type of the secure framework, there would be still two security layers against a successful attack;

1- The attacker does not have a signed certificate, therefore, its publisher access to the topic is denied by the system.

2- The attacker's attempt to publish data to the topic is failed because it needs the shared key to be able to communicate with participants in this domain.

Although the admin console and attacker's access to the topic are denied, the DDS creates the same framework with the same topic name and domain ID for these participants, which is different from the secured structure. This robustness against cyber threats makes this communication channel secure enough in such a way that real R-GOOSE messages are subscribed by the target IED as shown in Figure 10.6b, where the output follows the input signal.

\subsection{Secure Sequence Hopping Algorithm for Routable Communications}

Although the developed MRGM framework and DDS security algorithm could provide a fast and secure platform for routable communications in power systems, the dependency of the security algorithm to a specific middleware extremely restricts this framework for users who utilize the DDS communication middleware. Therefore, in this section of this dissertation, we propose a new approach for data integrity and security of critical routable communications in the smart power system, which is independent of communication middleware and is very fast to address communication challenges. 


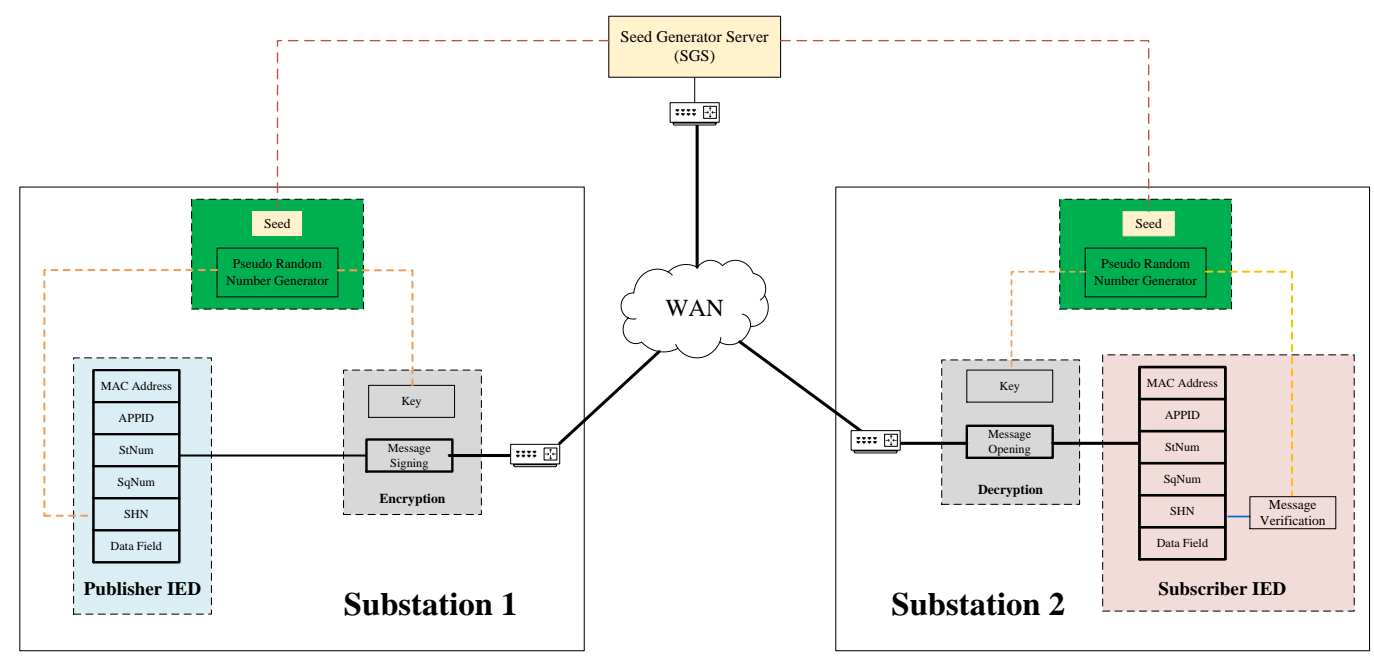

Figure 10.7: SSHA for R-GOOSE message communication

This new approach is called the SSHA. The SSHA introduced for data integrity and security when messages are broadcasted into the WAN. Figure 10.7 demonstrates the developed SSHA. As can be seen, two communication channels are required. The first one is an encrypted connection (e.g., a SSL channel), which is used by a SGS to communicate with both participants over an encrypted channel. The aim it to exchange random seed numbers and synchronizes random number generators between publisher and subscriber. This seed will be used by the PRNGs algorithm to produce two unique and non-reputable random numbers for each message; The first one will be attached to the message (sequence hopping number) to prevent replay attack, and the second one is used to sign the message through a light symmetric encryption algorithm to ensure data integrity.

The second communication channel is used to route and transfer the signed message to its destination. Since the receiver has the same seed random number, it will produce the two same random numbers (random numbers used by the publisher), once it receives a new message. Therefore, if the message is not manipulated, the decryption algorithm quickly finds the content of the message by using its second random number (Key) to decrypt the 
message. The process continues by checking the first random number of the receiver with the sequence hopping number attached to the GOOSE message.

If the decryption is successful and the sequence hopping number is the same, the message is verified, and the subscriber reacts to it. Otherwise, it is rejected. It is worth mentioning that the SGS can change the seed number periodically. Regarding the security level of the application, this period could vary from some milliseconds to days, weeks, etc.

\subsubsection{SSHA Development}

In this work, we propose to use the DDS as a standard for publish/subscribe message. Using the security mechanism for DDS, we can create an encrypted channel for communication between the SGS and publisher/subscribers in two different substations. Figure 10.8 shows our developed framework for implementing the DDS to transfer GOOSE messages between two substations in different networks and an encrypted channel for synchronizing the seeds for all pseudo random number generators. After receiving the synchronized seed number by DDS participants, the following steps are required:

a) The first step is to create a GOOSE message, for example, a regular pulse between participants or a trip/blocking signal form IED and add the first random number (generated by pseudo random number unit) to it as sequence hopping number.

b) To use the DDS, we need to convert the GOOSE message to the DDS message, for this purpose, we need to follow these subsections:

1) Subscribe the GOOSE message,

2) Sign the message using the second random number generated by the pseudo-random number unit using a light encryption algorithm (e.g., XOR with very low latency). 
3) Convert the encrypted message to the DDS message,

4) Publish the DDS message to a pre-defined topic in the LAN at substation1.

c) By receiving the DDS message, the DDS Routing service is deployed to route the message to its destination over the WAN.

d) DDS subscriber receives the message at substation2 and goes through the following steps:

1) Using the second random number generated by the pseudo-random number unit in Substation2 to decrypt and read the message.

2) Converts the received message to a GOOSE message and publishes it in its local network.

3) The subscribed IED will receive the message, compare its sequence hopping number with the first random number generated by its pseudorandom generation unit.

4) If the message is verified, the subscriber IED reacts to it. Otherwise, the message is ignored.

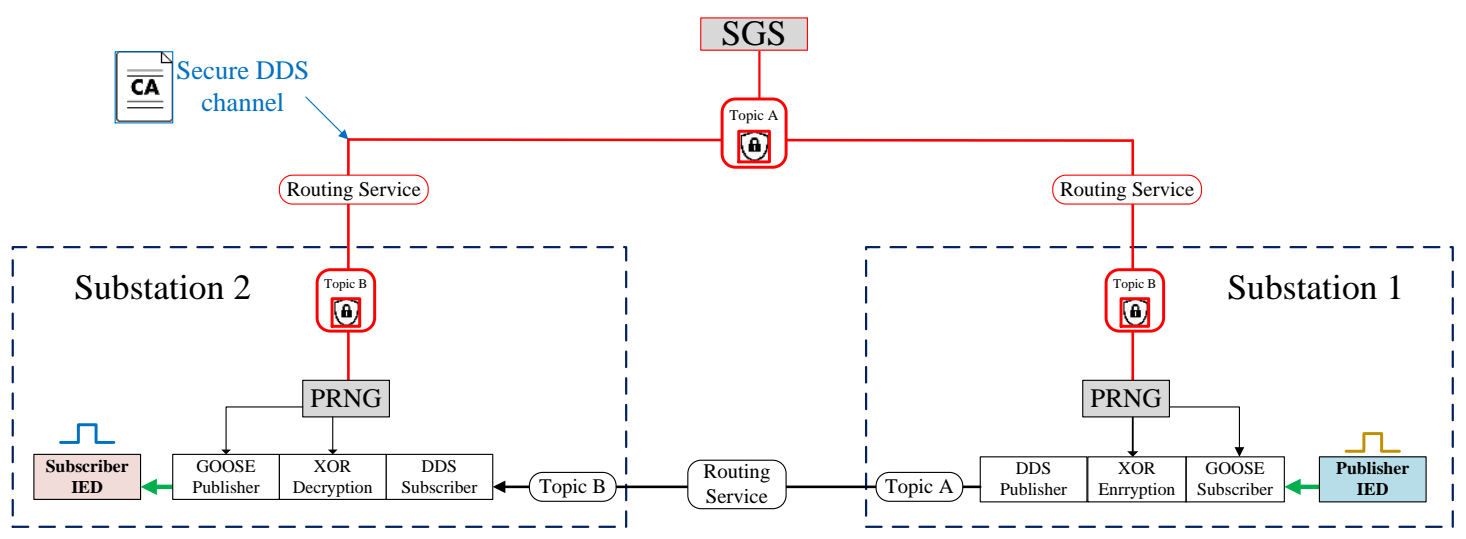

Figure 10.8: DDS-Based Communication Framework for SSHA. 


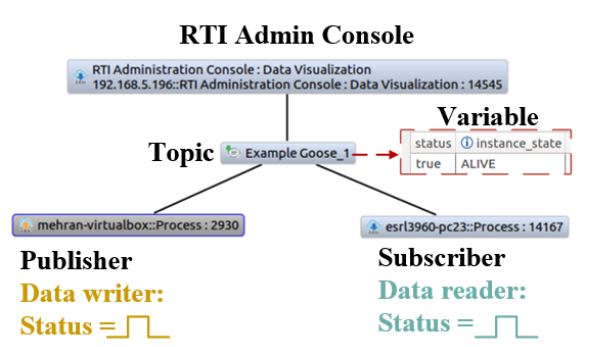

(a)

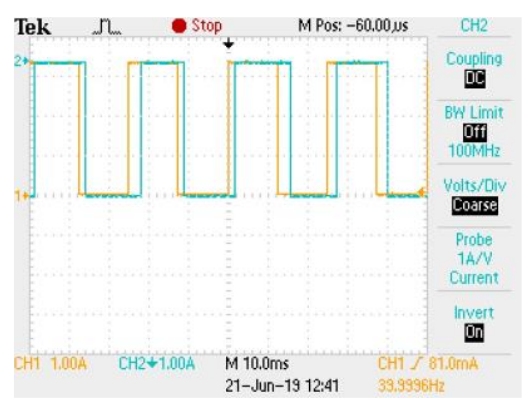

(b)

Figure 10.9: DDS-based MRGM with SSHA security algorithm.

\subsubsection{SSHA latency measurement}

We modified the developed MRGM framework to measure the latency of the developed SSHA algorithm. For this aim, instead of using DDS secure in PC1 and PC2 of the setup shown in Figure 10.4, we deployed the SSHA algorithm to sign messages in Substation 1 and read it in Substation2 using the XOR algorithm. Since the XOR algorithm is very lightweight and has very low latency (coupe of microseconds), it is expected that the SSHA should be faster than the DDS security algorithm as experimental results verify it. The results shown in Figure 10.7a demonstrate the DDS domain, and Figure 10.7b shows the recorded samples for this case study. For 1000 samples, the minimum and maximum delay were 1050 and $2840 \mu$ s, respectively. The average value was around $2135 \mu$ s. Hence, the average latency of SSHA in this test setup is about $35 \mu \mathrm{s}$.

It is worth mentioning that this latency highly depends on the processor speed, the size of the message, etc. However, the developed SSHA show much lower latency compared with the DDS security algorithm for the same size of the message transferred over the same communication framework. Moreover, the SSHA can be used by any other communication. 


\subsection{Summary}

In this chapter, the security issue of critical time-sensitive routable communications in the smart grid was addressed by two algorithms. At first, the DDS security algorithm was deployed to the MRGM framework, and its latency was measured using an experimental setup. Afterward, a bad data injection cyber-attack was lunched on this framework to compare the MRGM framework without DDS security measure with the one with the DDS security algorithm. The results showed that the DDS security algorithm is fast and secure enough for this type of communication in power systems. In fact, this approach is a feasible secure framework for R-GOOSE messages while the DDS is available as a communication middleware. However, to address the security of R-GOOSE messages while the DDS is not available, we developed the SSHA as a lightweight security algorithm, which presents a unique Key management mechanism for time sensitive and critical communication in smart grids. The SSHA is based on a sequence hopping algorithm. This security method is independent of the communication middleware and is very fast and reliable for data integrity and security of critical communications in smart grids. 


\section{Chapter 11 Application of Critical Communications in Modern Power}

\section{System}

In Chapter 9, a DDS-based communication framework was developed for multicast RGOOSE messages over the wide-area network for effective substation-to-substation and substation to control center communications. In Chapter 10, two different security algorithms were developed and deployed to ensure data integrity and security for this critical communication in modern power system. However, the advantages of developing this secure communication in modern power system was not highlighted in last chapters. In this chapter, we are going to show how secure routable communications can enhance the power system reliability and decrease the protection costs.

\subsection{Dealing with fault current in power systems}

In power systems, it is necessary to remove the fault current quickly to avoid negative consequences due to fault occurrence in different parts of the network. The protection system is responsible for this issue and many protection devices and several methods are used to limit and remove the fault current from the system. Depending on the system under study, voltage level and available equipment, different protection scheme and devices are deployed.

\subsubsection{Conventional protective devices}

Beside the fuses, which are mostly used for protection of radial distribution systems, protective relays along with circuit breakers are used to protect the transmission and subtransmission systems against different types of faults [198]. These relays are equipped with CVTs to measure actual current and voltage of the system. 
Table 11.1: Breaking capacity of CBs in different voltage levels.

\begin{tabular}{|c|c|c|c|c|}
\hline Circuit breaker type & \multicolumn{3}{|c|}{$L T B, D C B \& W C B$} & $H P L$ \\
\hline Rated voltage ( $\mathrm{kV} \mathrm{rms}$ ) & $72.5-300$ & $362-550$ & 800 & $72.5-1200$ \\
\hline Rated current (kA) & $3.15 / 4$ & $3.15 / 4$ & 4 & $5 / 6.3$ \\
\hline Breaking capacity $(k A)$ & $31.5 / 40 / 50$ & $40 / 50 / 63$ & $40 / 50$ & $0-80$ \\
\hline Break time (cycles) & 2.5 & 2.5 & 2.5 & 2 \\
\hline
\end{tabular}

Regarding the relay type and its applications, there are several fault detection algorithms and protection scheme, which can be deployed in protection plan such as overcurrent protection, distance protection etc. By detecting and classifying the fault, relay sends the trip signal to the circuit breaker to isolate the faulted area from the rest of system.

The ability of circuit breakers to break the fault current is called $\mathrm{BC}$ of the circuit breaker. As reported in [199], usually there are several standard BCs for different voltage levels by different types of circuit breakers in power systems shown in Table 11.1. In this Table, LTB stands for Live tank CB (with low operating energy), DCB is used in simplified substations and provides the functionality of both circuit breaker and disconnector within a single unit. DCB is more available with lower cost and it is reduced space, WCB is withdrawable circuit breaker (fulfills all requirements for a circuit breaker as well as for a disconnector) and HPL, for extra high performance and high current capability. The HPL circuit breakers provide higher breaking capacity and are very expensive.

The rated fault currents for each voltage level demonstrate the breaking capacity of standard circuit breakers for that voltage level. As can be seen in Table 11.1, the maximum standard and conventional BC for a $400 \mathrm{kV}$ system is $63 \mathrm{kA}$. It should be noted that the maximum duration of fault current is the maximum time, which the component in the system can withstand the rated fault current before melting. Although this time is highly dependents on the weather parameters (temperature, humidity etc.), usually the worst weather conditions are considered to determine this time interval. 


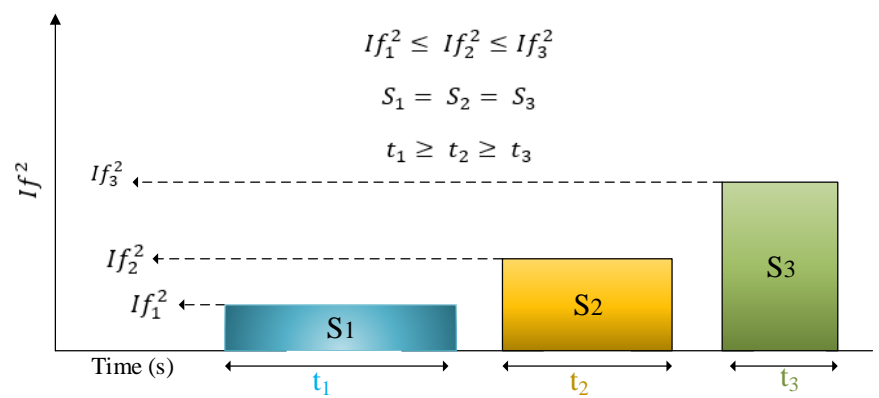

Figure 11.1: Maximum fault current and its maximum duration time

Therefore, any rated fault current in the system should be removed before reaching the pre-defined allowed time. Although for fault currents which are below the maximum rated current, this time interval is increased, for the faults with higher magnitudes the maximum duration time is decreased. Even though these changes are not entirely linear, we can use the equality of energy surfaces to calculate the maximum duration for fault currents different from the rated current as shown in Figure 11.1.

\subsubsection{Limiting the high level fault current}

Growing the power systems and connecting power networks together along with increasing the generation capacity to serve the electricity demand with high reliability have highly affected the protection system by increasing the short circuit level at different voltage levels. To deal with very high fault currents, which are more than $\mathrm{BC}$ of circuit breakers in the system, new circuit breakers with higher BCs, must be used which in most cases are very expensive or it is not possible because the fault current is higher than maximum BCs for that voltage level. In these situations, power system planners try to reconfigure the system or limit the fault current using different fault current limiters such as high impedance transformers, current limiting rectos or superconductor FCLs [139] and [198]. In all mentioned methods, the goal is to limit the short circuit current to be less than $\mathrm{BC}$ of circuit breaker and as a result, a successful relay trip can clear the fault. 


\subsection{HV Transmission system protection}

Before describing the developed protection scheme in dealing with very high fault currents in HV transmission systems, we need to evaluate the power system from different points of view such as the percentage of high fault current in high voltage transmission systems, the conventional protection scheme in $\mathrm{HV}$ transmission level, functionality of circuit breakers and finally the application of IEC 61850 standard in protection systems.

\subsubsection{Fault statistics}

Based on the literature and actual statistics from different countries, the average number of the faults in HV transmission system has been decreased in recent years due to advanced technology. For example, the average number of faults per $100 \mathrm{~km}$ of transmission lines in Denmark, Finland, Norway and Sweden decreased from 0.5057 in 1996 to 0.33 in 2009 [200]. Another statistical analysis in Qingyuan of Guangdong Province in China because demonstrates a dramatic reduction in the number of transmission lines tripping between 2013 to 2016 from 100 to 60, respectively [201]. The cause of faults is highly depended on the geographical location. For example, the in aforementioned reference, the lightening is the major cause of transmission line tripping which contain around 80 to $90 \%$ of protection system's actions during those years.

This reduction in the tripping frequency is because of improvement of protection measures on transmission lines. The South African transmission system study demonstrates that the primary causes of faults are bird streamers, lightning and fires, with $38 \%, 26 \%$ and $22 \%$, respectively [202]. The improvement in the system equipment, by installing bird guards, resulted in a significant reduction in the average fault frequency for these lines from 2.38 faults $/ 100 \mathrm{~km} /$ year to 1.35 from 2000 onwards. It can be understood 
from these examples that improvements in the system equipment as well as protection system in recent years led to improve the system reliability incises by declining the number of interruptions for customers. As reported in 2017 annual electric reliability report of the Pacific Gas and Electric company (PG\&E), the company that provides natural gas and electricity to most of the northern two-thirds of California in United States, reliability indices related to service interruption issues had linear decreasing trend for the past 10 years [203]. This reference reports that the System Average Interruption Frequency Index (SAIFI) for the transmission system has declined from 0.166 interruption/customer/year in 2008 to 0.085 in 2017. A similar deceasing trend has also been reported for the System Average Interruption Duration Index (SAIDI) from 23.8 hour/year in 2008 to 7.3 in 2017.

It is worth mentioning that the average value contains all types of fault in the system including single-phase-to-ground, phase-to-phase, phase-to-phase-to-ground, three-phase and three-phases-to-ground. As reported in IEC WG29 doc, for transmission systems with the voltage level between 300 to $500 \mathrm{kV}, 83 \%$ of faults are single-phase while two-phase and three-phase faults are around $14 \%$ and 3\%, respectively. Furthermore, all faults may include fault resistance, which plays a main role in fault current calculations. It means that the probability of very high fault currents within specific part of transmission line is too low and it may be occurred if for example the staff could forget to remove the temporary three-phase ground before switching on the considered network section.

It may also happen if the CBs are not replaced by higher breaking capacity according to system expansion planning and maximum probable SHC level, because of many reasons such as economic issues or maintenance schedules. In these situations, and because of this fact that the probability of very high fault currents in the system is too low, implementing 
the costly methods such as installing FCLs or replacing the circuit breakers with higher BCs (which are usually expensive) is not an optimum solution for this kind of systems and faults. Therefore, developing another protection scheme, which can address this specific protection is the contribution of this chapter of dissertation.

\subsubsection{Fault clearing time}

Fault clearing time is the time interval between the fault inception and the fault clearance. Fault clearing time consists of several sections as follow:

\subsubsection{Relay operating time}

It is generally the time interval between receiving measurement samples form CVTs and analyzing (fault detection and classification) and sending control signals to circuit breakers or other relays. Regarding the methods which are used for fault detection and classification, this time interval varies between 2 to $10 \mathrm{~ms}$ for detection the fault and up to $30 \mathrm{~ms}$ for fault classification [200]. In addition to this time interval, the protection scheme may also add more delay in this step to coordinate the protective relays reactions to a fault current. For example, in $400 \mathrm{kV}$ system, usually the main and backup protections are Distance-Distance. It means that not only the main protection scheme is based on distance protection in transmission system, but also the backup protection is distance protection with more delay time. Usually the distance protection defines three or four protection zone, which are called zone 1 to zone 4 . Zone 1 is considered as the main protection zone in distance protection which cover up to $85 \%$ of transmission line' length. This zone is designed to react to the fault currents immediately, therefore no time delay is implemented for this zone. The rest zones (zone 2, zone 3 and zone 4) are backup protections and are designed to react to the fault current if the main protection fails to clear the fault current. 


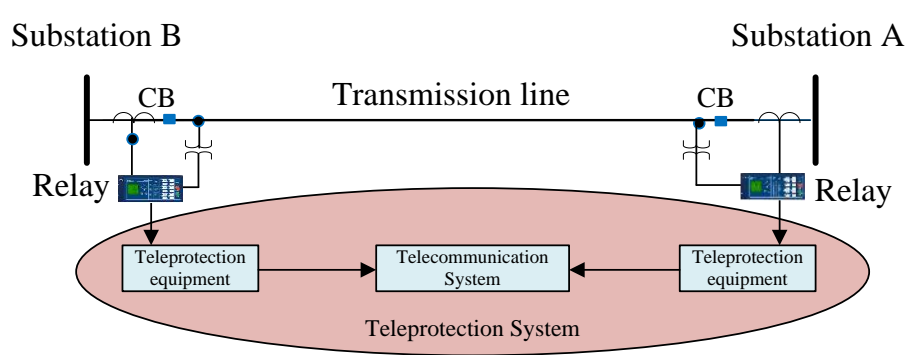

Figure 11.2: Telecommunication in the power system.

The time delay for these zones is usually between 400 to $500 \mathrm{~ms}$ [204]. It means that zone 2 will send the control signals by 400 to $500 \mathrm{~ms}$ delay regarding the zone 1 and zone 3 will add another 400 to $500 \mathrm{~ms}$ to delay of the zone 2, to send the command signals.

\subsubsection{Teleprotection Systems Transfer Time}

A classic protection system for a high voltage transmission line is shown in Figure 11.2. As it can be seen, in addition to protective relays, teleprotection equipment and telecommunication system is required. In fact, teleprotection equipment is an interface between relay and telecommunication system which uses different types of communication links such as PLC links, Fiber-optic links, Copper wires/pilot cables, Microwave-radio links or IP based network [205]. It can communicate through Ethernet within a substation using publisher-subscriber protocol instead of using hard-wire connections. In addition to communication in data link of OSI model (layer 2), IEC working groups have extended IEC 61850 standard for SS2SS communication through WAN which actually occurs in layer 3 of the OSI model (Network or IP layer). The standard defines the maximum latency for communication within a substation by $4 \mathrm{~ms}$ and $10 \mathrm{~ms}$ for SS2SS communication. More latency for the S2S communication is because of the fact that messages should pass from layer 2 (data link) to layer 3 (network layer) and use routing service to find destination in the network. If GOOSE message is sent between substations, it is called R-GOOSE. 
Table 11.2: Time delay for each section of the protection systems.

\begin{tabular}{|l|l|}
\hline \multicolumn{1}{|c|}{ Section } & \multicolumn{1}{c|}{ Delay } \\
\hline Fault detection & $2-10 \mathrm{~ms}$ \\
\hline Fault classification & Up to $30 \mathrm{~ms}$ \\
\hline Sending signals within substation (GOOSE) & Up to $4 \mathrm{~ms}$ \\
\hline Sending signals Between substation (R-GOOSE) & Up to $10 \mathrm{~ms}$ \\
\hline Breaker operating time & Up to $50 \mathrm{~ms}$ \\
\hline Distance protection delay Zone 1 & 0 \\
\hline Distance protection delay Zone 2 & $400-500 \mathrm{~ms}$ \\
\hline Distance protection delay Zone 3 & $400-500 \mathrm{~ms}$ in addition to Z2 \\
\hline
\end{tabular}

\subsubsection{Circuit Breaker Breaking Time}

This latency in FCT is also called total break time of CB consists of opening time along with arcing time. Since fast performance of $\mathrm{CB}$ is required especially in HV systems (because it directly affects the system stability), the maximum breaking time for CB in 400 KV system should be within 2.5 cycles. It means that in a $50 \mathrm{~Hz}$ system, the CB should be able to open its contacts in less than $50 \mathrm{~ms}$ [206]. Table 11.2 shows the summary of this section in terms of time delay for each section of protection system in a typical $400 \mathrm{kV}$ system. As shown in this Table, the time delay for distance protection in the zone 2 is typically between 400 to $500 \mathrm{~ms}$, and additional 400 to $500 \mathrm{~ms}$ is usually defined for time delay of zone 3 and 4 .

\subsection{The developed High Current Protection Scheme}

Figure 11.3 shows the architecture of protection scheme where the IEC 61850 is used for data exchanging between protective relays within or between substations. As it can be seen, the protective relays in each substation are communicating though a LAN and a fast network switch is used to send/receive signals to/from other relays in this station. The exchanged data could be SMV messages from different MUs in this substation or control command (GOOSE messages) such 


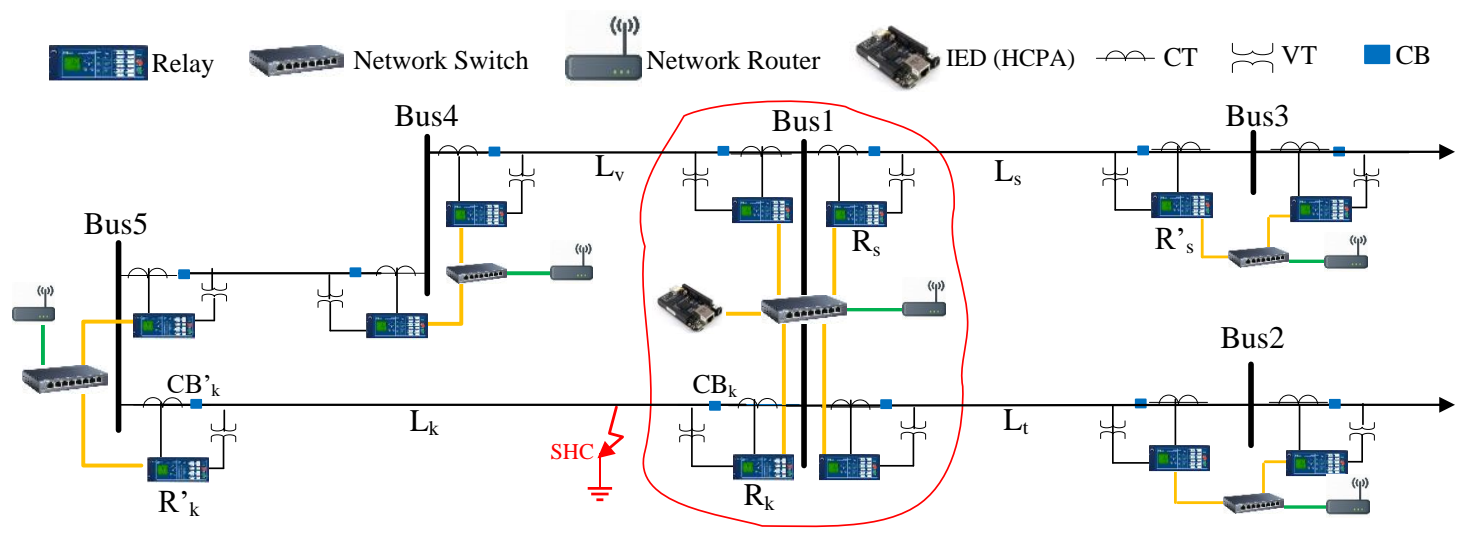

Figure 11.3: Protection System architecture based on IEC 61850.

as tripping, blocking or reclosing signals. In addition to the LAN for communication within each substation, the WAN is also deployed for inter-substation communication. Each substation is equipped with a network router which can communicate with other networks to exchange data over the internet network. For this type of communication between relays in different substations, data should pass from layer 2 (data layer) to layer 3 (network layer) and use the IP to find their destinations in the network.

The GOOSE messages which are sent/receive through IP are called R-GOOSE messages. These messages are used to exchange tripping, blocking or reclosing signals with relays at the other end of transmission line in another substation or control center. Since the GOOSE and SMV messages defined in IEC 61850 use the RTPS to send/receive data, an IED with proper subscribers connected to network switch can receive all published data by all relays in the network and also it can publish messages to different relays within its local substation or other relays in another substation through appropriate logical nodes defined in the standard as shown in Figure 11.4.

In this study, this IED is called the HCPA which can add an intelligent protection scheme to the current protection system to deal with very high fault currents higher than breaking capacity of circuit breakers in the system. 


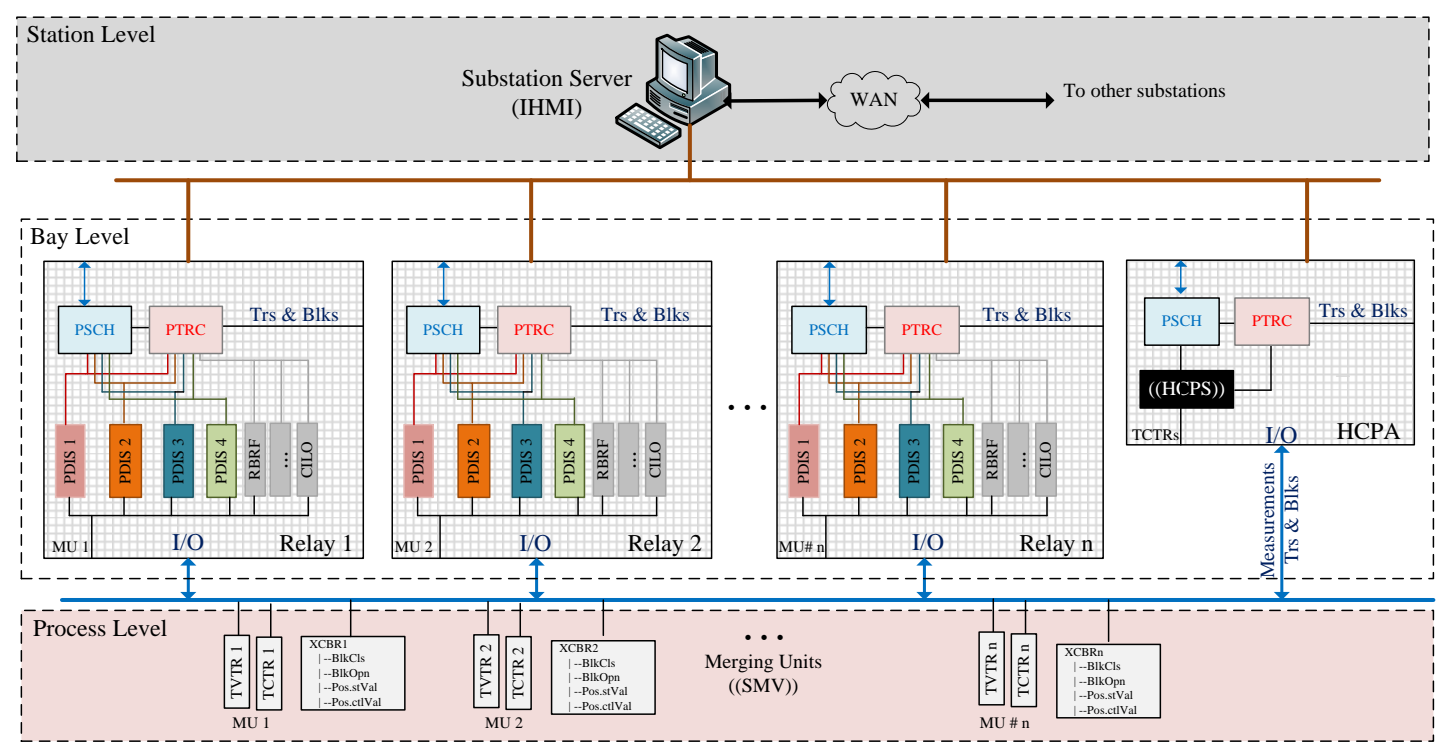

Figure 11.4: The architecture of the HCPA based on IEC 61850.

For this purpose and as shown in Figure 11.4, the HCPA should be equipped by SMV subscribers to listen to published values by TCTRs (logical nodes for current measurement of the MUs) within the substations. At the same time, the HCPA is listening to the distance protection logical nodes (PDIS) of all relays in the substation to map the tripping orders by executing the HCPS if fault with very high current is occurred. This figure also demonstrates the three levels of substation automation; the station level with the human and machine interface (HMI) and the gateway $(\mathrm{GW})$, the bay level with its units (BU) for protection and control, and the process level near the switchyard.

\subsection{Flowchart of the developed HCPS}

The developed HCPS supervises the operation of distance protection units of all relays within the substation. Figure 11.5 shows the flowchart of developed HCPS which is implemented by HCPA in a substation where very high fault currents is probable. From Figures 11.4 and 11.5, it can be seen that the HCPA is subscribing to MUs and distance protection units of all other relays in the substation. 


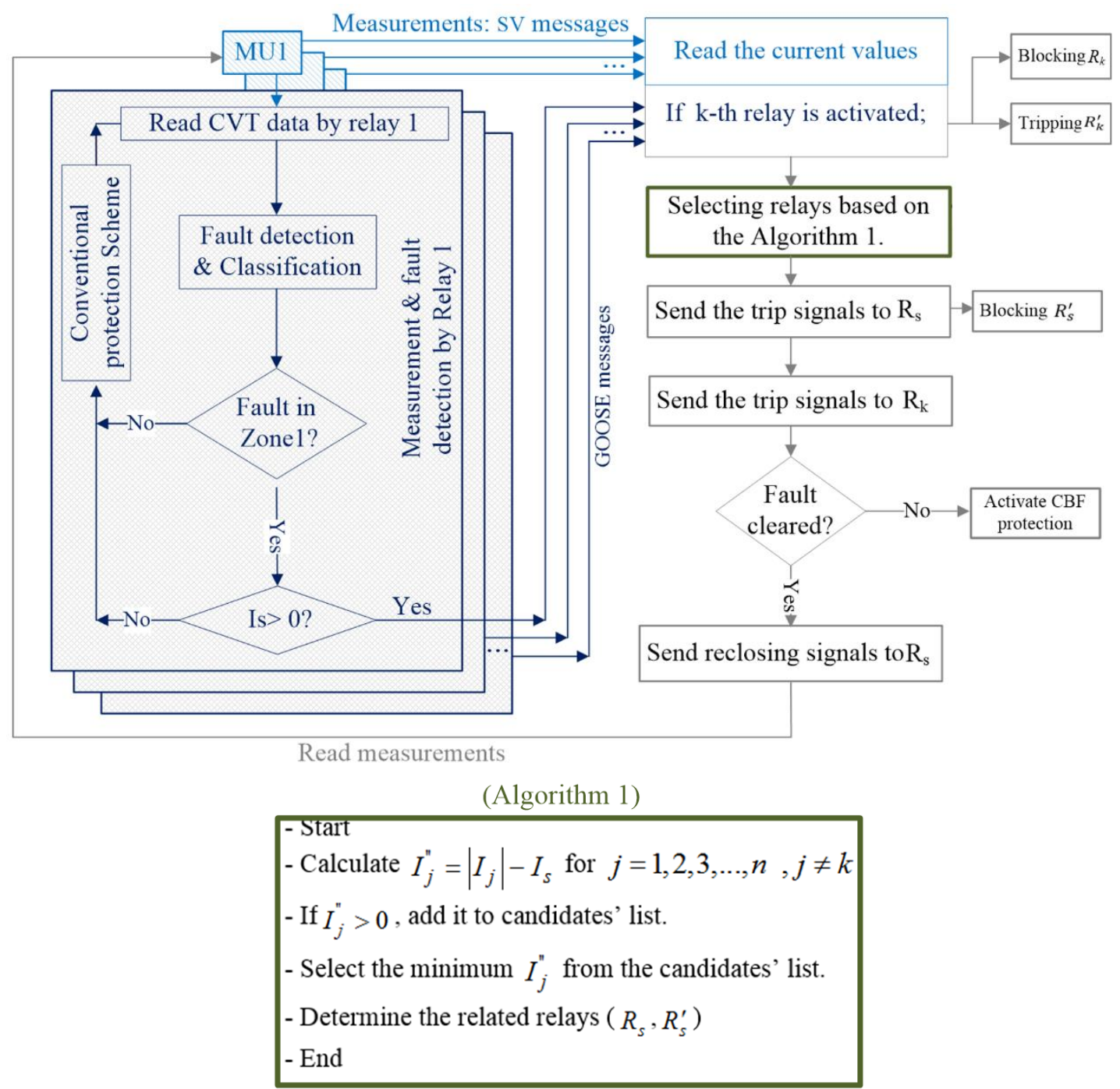

Figure 11.5: Flowchart of the developed HCPS and the Algorithm of HCPS activation

To more clarify the developed algorithm, let's assume the HCPA is located at Bus 1 and SHC is occurred in $\mathrm{k}^{\text {th }}$ line in the system $\left(\mathrm{L}_{\mathrm{k}}\right)$ very close to $\mathrm{kth}$ relay $\left(\mathrm{R}_{\mathrm{k}}\right)$ as shown in Figure 11.3. If $\mathrm{k}^{\text {th }}$ relay senses a fault in zone 1 of its distance protection, and the SHC is higher than the BC of the $\mathrm{k}^{\text {th }}$ circuit breaker $\left(I_{k}^{m}\right)$, the HCPS is activated and immediate blocking signal is sent to the $\mathrm{CB}_{\mathrm{k}}$ and at the same time, tripping signals are sent to $R_{k}^{\prime}$ at the other end of the line to stop feeding the fault from other substation. The HCPS evaluates the magnitude of the fault current and compares it with $I_{k}^{m}$ of $\mathrm{CB}_{\mathrm{k}}$ to calculate the surplus 
current $\left(I_{S}\right)$ by $(11-1)$.

$$
I_{s}=\left|I_{f}\right|-I_{k}^{m}
$$

For very high fault currents, we will see $I_{S}>0$, it means that although the distance protection sends the trip signal to $\mathrm{CB}_{\mathrm{k}}$, it can't open its contacts and break the fault current. In this situation backup protection is activated which will result in huge outages due to zone 2 operation of all relays feeding this fault.

To avoid this interruption and clear the fault in shortest possible time without installing the FCLs in this system, the HCPA is used to send cascading blocking, tripping and reclosing signals to other relays in this system to minimize the service interruptions. At first, the blocking signal is sent to $R_{k}$ because it can't stop the fault current and to avoid sending CBF to the busbar protection, while a trip signal is publisher to $R_{k}^{\prime}$ to stop feeding the fault from other end of the line quickly. At the same time, it evaluates the fault currents measured by other MUs in this substation and executes the algorithm 1 of the flowchart as shown in Figure 11.5 to find the best relays for tripping.

As it can be seen in this algorithm, the surplus current is compared with measured currents by CTs in this substation $\left(\mathrm{I}_{\mathrm{j}}\right)$, if the comparison result $\left(I_{j}^{\prime \prime}\right)$ is positive, the related relay $\left(R_{j}\right)$ would be a candidate for tripping. Between these candidates, the one with the minimum value is selected and its related relay is called $\left(\mathrm{R}_{\mathrm{s}}\right) . R_{S}^{\prime}$ stands for the relay which is located at the other end of transmission line where the $\mathrm{R}_{\mathrm{s}}$ is located. This selection means that if this relay breaks its own fault current, the $I_{s}$ will be negative which means that $\mathrm{CB}_{\mathrm{k}}$ can now break the fault current because it is less than its breaking capacity. To do this, the HCPA sends the trip signal to $R_{s}$ and at the same time blocking signal to $R_{s}^{\prime}$. 


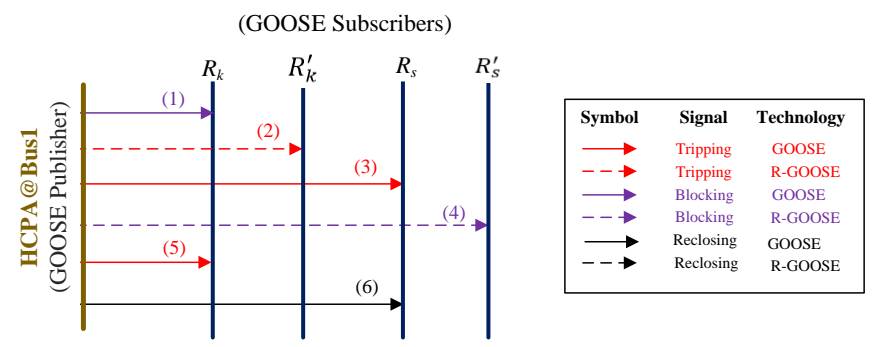

Figure 11.6: GOOSE message traffic created by HCPA at Bus 1.
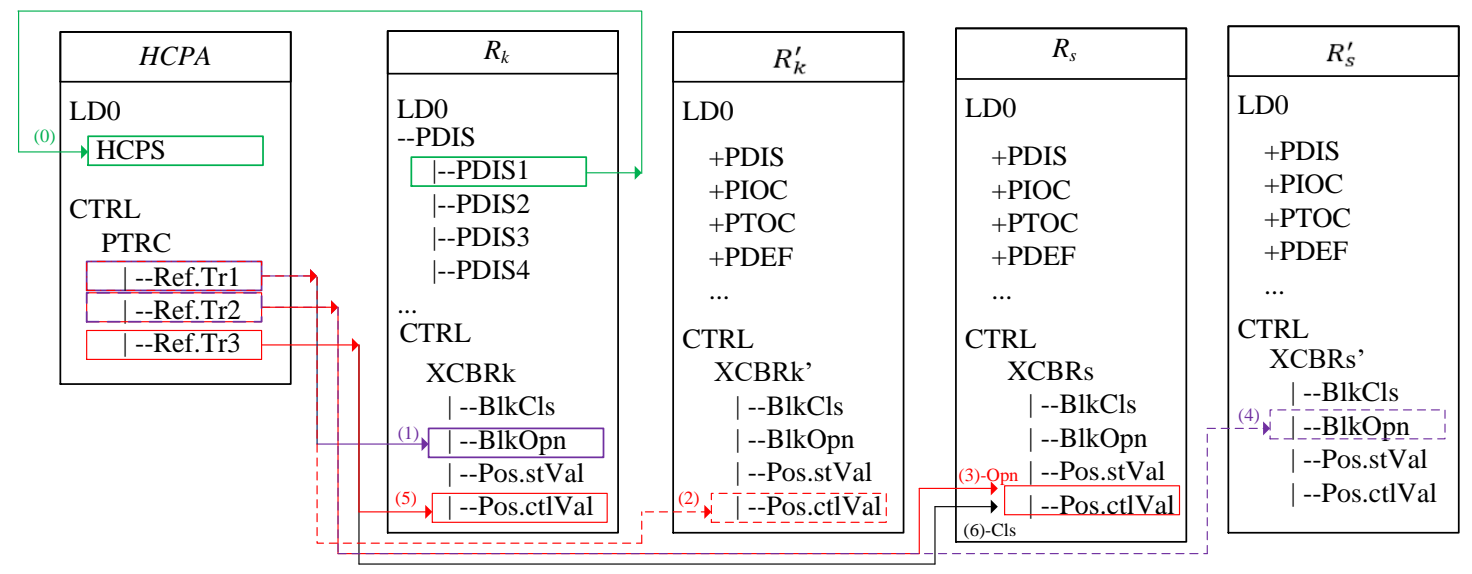

Figure 11.7: GOOSE mapping between HCPA at Bus 1and IEDs

Afterward, the tripping signal is sent to the main relay close to fault (Rk) and after fault clearing, a reclosing signal is sent to pre-tripped relay (Rs) to return it back to the service. The GOOSE messages traffic created by HCPA at bus 1 is shown in Figure 11.6. As it is shown in this figure, the HCPA is a GOOSE publisher and different relays in the system may subscribe to published messages regarding the protection requirements.

Figure 11.7 demonstrates the GOOSE mapping between HCPA and other relays to break this fault current. The HCPS is initiated by receiving the signal from PDIS1 (logical node for zone 1 of distance protection) of relay k. It goes through the flowchart steps and selects appropriate circuit breakers to send cascading tripping, blocking and reclosing signals. The logical nodes of each IED in this fault situation along with data objects and control commands are shown in Figure 11.7. As can be seen, after HCPS activation, the PTRC (protection trip conditioning), which is a logical node that creates binary outputs, in 
the HCPA sends a blocking signal to the XCBR (logical node of a circuit breaker) in relay $\mathrm{k}$ to block opening this $\mathrm{CB}$. The process continues as described in Figure 11.5 until the fault is cleared and $\mathrm{L}_{\mathrm{k}}$ is isolated. Furthermore, it can be understood from these figure that the HCPA is running an additional protection scheme over the conventional distance protection to avoid large service interruption in the system.

\subsection{Flexibility and reliability of the developed HCPS}

The flexibility of the developed HCPS cloud be demonstrated when different busbar arrangements exist. It should be noted that the developed HCPS could be implemented to any busbar arrangement by defining an appropriate switching action based on the busbar configuration. It means that regarding the number of the circuit breakers which are receiving commands from HCPA is different. For example, if the busbar has the one-anda-half breaker arrangement, the control commands for each transmission line should be sent to two breakers, simultaneously.

In addition, and because of the low probability of such very high fault currents in power systems, it may be concluded that auto-reclosing is not necessary for this supervisory protection. However, the auto-reclosing logic can be implemented in this algorithm by just adding a logical node related to auto reclosing to the HCPA. It would be a little complicated because auto-reclosing in this situation means switching onto very high fault current. Therefore, the auto-reclosing scheme should operate same as HCPA tripping order but in opposite direction. It means that after breaking the fault current by the main relay $\left(\mathrm{R}_{\mathrm{k}}\right)$, the pre-selected relay (Rs) should be opened first, and then $R_{k}$ should be closed to see if the fault exists or not. Generally, implementing the reclosing logic is possible if the probability of extreme fault currents is increased. 
The reliability of communication network is one of the most important issue when the IEC 61850 is implemented for the SA. If the communication network is non-redundant, some inherent communication redundancy is provided using a ring of switches connected to IEDs with a single link. The system may include two independent subsystems where each IED communicates with two networks through two separate ports. Developing several redundant networks could significantly increase the reliability of the communication network as discussed in [207]. Meanwhile the communication network could be reliable enough, and the probability of extreme fault current in the power systems is too low, losing the communication network when an extreme fault current is occurred, would be mathematically negligible and practically impossible. If it happens, the backup protection in zone 2 of other transmission lines would be activated to clear the fault from the system by a larger number of equipment outages as we will discuss about it in the next section.

Numerical results in the next section will obviously demonstrate the advantages of developed HCPS in dealing with very high fault currents in the system in terms of minimum fault clearing time, minimum number of transmission line outages and more stability margin.

\subsection{Numerical results}

This section contains simulation results for developed HCPS in a $400 \mathrm{kV}$ power system with very high fault current due to some three-phase to ground faults. Power system simulation and propose protection scheme along with conventional distance protection are modeled in DIgSILENT PowerFactory 2019. Quasi-dynamic simulation is run to show the dynamic behavior of the power system in time domain for electromechanical and electromagnetic transient simulations. 


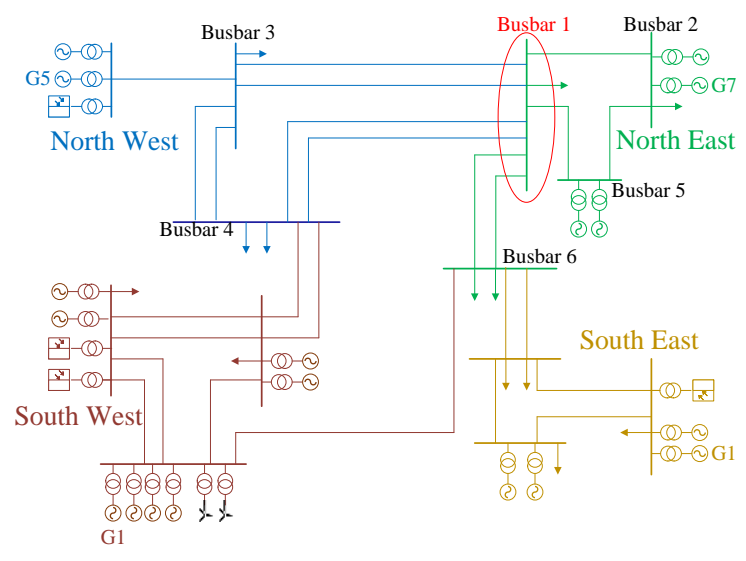

Figure 11.8: $400 \mathrm{kV}$ system under study

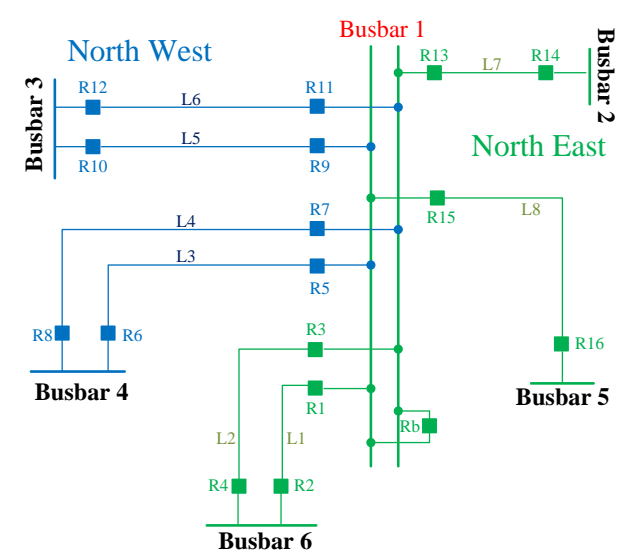

Figure 11.9: Busbar 1 in the $400 \mathrm{kV}$ system

To model the short circuit in this study, the complete short circuit method (superposition method) is used which is known as high accurate method for short circuit calculations. It compromises of three steps; first step is determination of steady-state condition before fault occurrence, in the second step the applied voltage to the fault location before short circuit and current distribution at the fault location are determined by negative sign. The last step superimposes both conditions to obtain a zero voltage at the fault location. In fact, this method assume that power flow is known before short circuit event and the power state is added to an amendment state before fault inception.

\subsubsection{Case 1}

Figure 11.8 shows the system under study in Case 1 or the base case. It is a $400 \mathrm{kV}$ power system containing four areas (indicated by four different colors) and different types of generation such as conventional synchronous generators, solar panels and wind turbines. The system parameters and specifications has been reported in PowerFactory 2019. Since this research focuses on protecting very high fault currents in power systems, we modified this system by adding some short circuit capacities to different parts of this system to reach high fault currents in some study cases. 


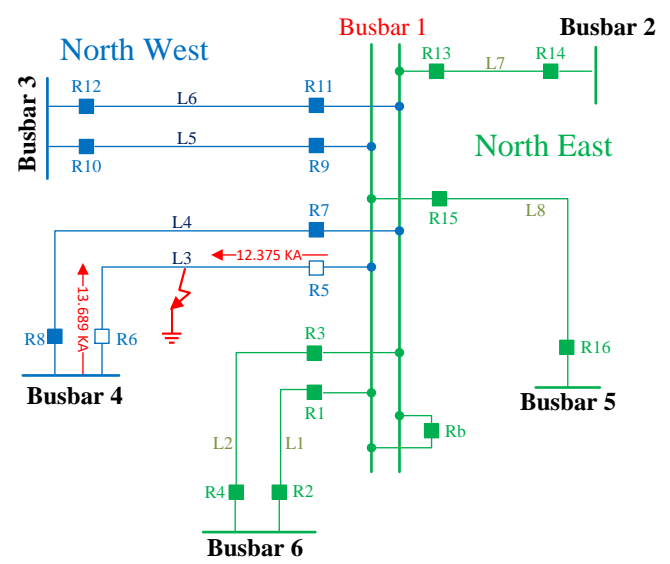

Figure 11.10: System status in case 1.

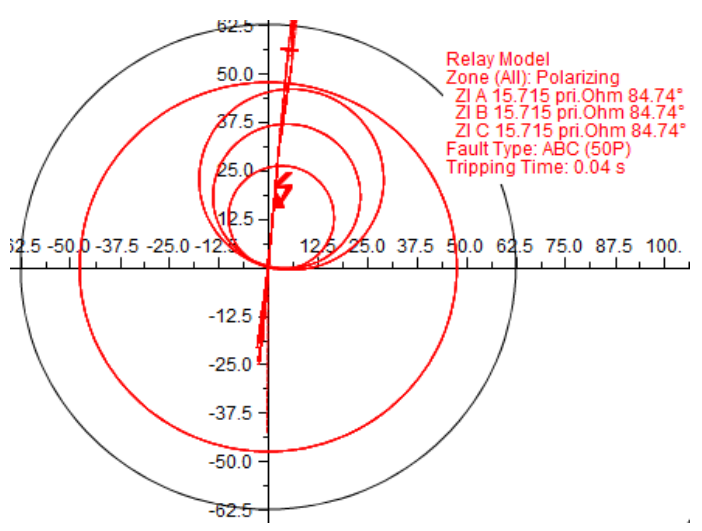

Figure 11.11: Distance characteristics of R5.

To perform the developed HCPS in this system, we just concentrate on a section of this system including Busbar 1 and all connected transmission lines to it. As shown in Figure 11.9 , this section compromises of 6 busbars, 8 transmission lines, 16 protective relays for transmission line, one relay for busbar coupling which are named Busbar 1 to Busbar 6, L1 to $\mathrm{L} 8, \mathrm{R} 1$ to $\mathrm{R} 16$ and $\mathrm{R}_{\mathrm{b}}$, respectively. This case shows the conventional distance protection results in dealing with an ordinary fault in the power system. In this case, a three phase to ground fault is modeled at the middle (50\%) of line 3 (L3) with zero fault impedance. The short circuit (SHC) currents in both sides of the line, for R6 and R5, are 13.669 and 12.375 $\mathrm{kA}$, respectively. Both R5 and R6 see the fault in the zone 1 and because the fault current is less than $\mathrm{BC}$ of $\mathrm{CBs}$, the HCPA will not be activated. It should be noted that $\mathrm{BC}$ of $\mathrm{CBs}$ for transmission lines is set to $63 \mathrm{kA}$. Therefore, instantaneous operation of zone 1 is expected for R5 and R6 and the fault is cleared in maximum $90 \mathrm{~ms}$ (FCT = $90 \mathrm{~ms}$ ). This delay is because of required time for fault detection and classification $(40 \mathrm{~ms})$ and circuit breaker breaking time $(50 \mathrm{~ms})$ as mentioned in Table 11.2. Figure 11.10 and 11.11 show the system status and distance characteristics of relays in this case. 


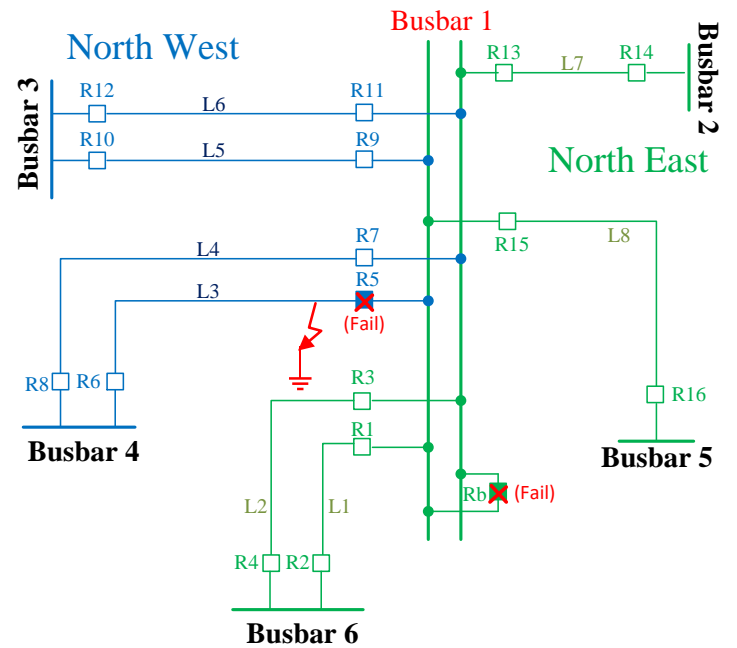

Figure 11.12: System status in case 2.
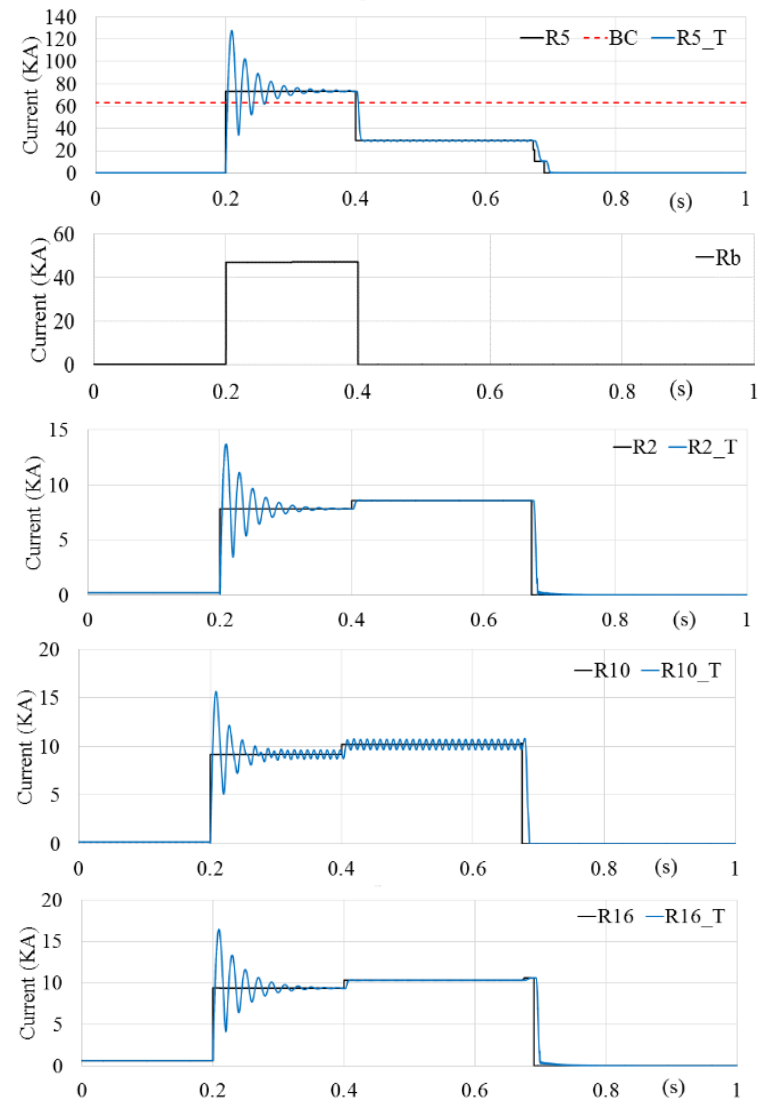

Figure 11.13: Quasi-dynamic simulation Results for Case 2. 


\subsubsection{Case 2}

In this case study, to simulate a very high fault current more than $63 \mathrm{kA}$, as shown in Figure 11.12, we model a three-phase to ground fault at the first 1\% of L3's length close to Busbar 1 and evaluate the conventional distance protection scheme in this situation. SHC calculations show that the fault current magnitude from Busbar 1 is $73.310 \mathrm{kA}$, which is more than $\mathrm{BC}$ of the circuit breaker of this line $(63 \mathrm{kA})$. This choice is just to simulate the fault current more than selected $63 \mathrm{kA}$ breaking capacity in this busbar. It should be noted that if the breaking capacity of this circuit breaker is just 40 or $53 \mathrm{kA}$, the more probable short circuit currents (between 40 to $60 \mathrm{kA}$ ) could pass the breaking capacity of this circuit breaker. In this case study, although R5 detects the fault in zone 1 and sends the trip signals to its own $\mathrm{CB}$ (CB5) and to R6 (relay at the other side of the L3, CB5 fails to break the fault current while CB6 is opened after receiving the trip signal. The continuation of fault current will cause a cascading relays' tripping as follow. The over current protection of $\mathrm{Rb}$ (busbar coupling relay) sees $46.955 \mathrm{kA}$ passing through its $\mathrm{CB}$ (we assume $40 \mathrm{kA} \mathrm{BC}$ for this circuit breaker), as a result it also fails to break the fault current. In this situation, R2, R4, R10, R12, R14 and R16 see the fault in zone 2.

To estimate TT for these relays, we should consider $40 \mathrm{~ms}$ for fault detection and classification, $400 \mathrm{~ms}$ for delay of zone 2 and let's assume maximum $50 \mathrm{~ms}$ for breaking time of CB located at R16. We assume that other CBs operate before the maximum $50 \mathrm{~ms}$ for breaking the fault current. Figure 11.13 shows the TT of some of these relays along with RMS and transient value of fault current passing through these relays. For example, (-R5) shows the RMS value for relay 5 while $\left(-\mathrm{R} 5 \_\mathrm{T}\right)$ demonstrates the transient value of this current. It should be noted that by sending trip signals by these relays, mutual relays 
of them will also get the tripping signals and as it can be seen in Figure 11.12, R1, R3, R9, R11, R13 and R15 will open their CBs too. Hence, the FCT for this fault using conventional distance protection scheme is equal to FCT in zone 2 and as it can be seen in Figure 11.13, the FCT in this case is $490 \mathrm{~ms}$ after the fault beginning. The summary of this protection is 15 switching actions which result in 8 transmission lines outage (L1 to L8) which is a huge outage in this system because North East is islanded from the rest of network and Busbar 1 is out of service.

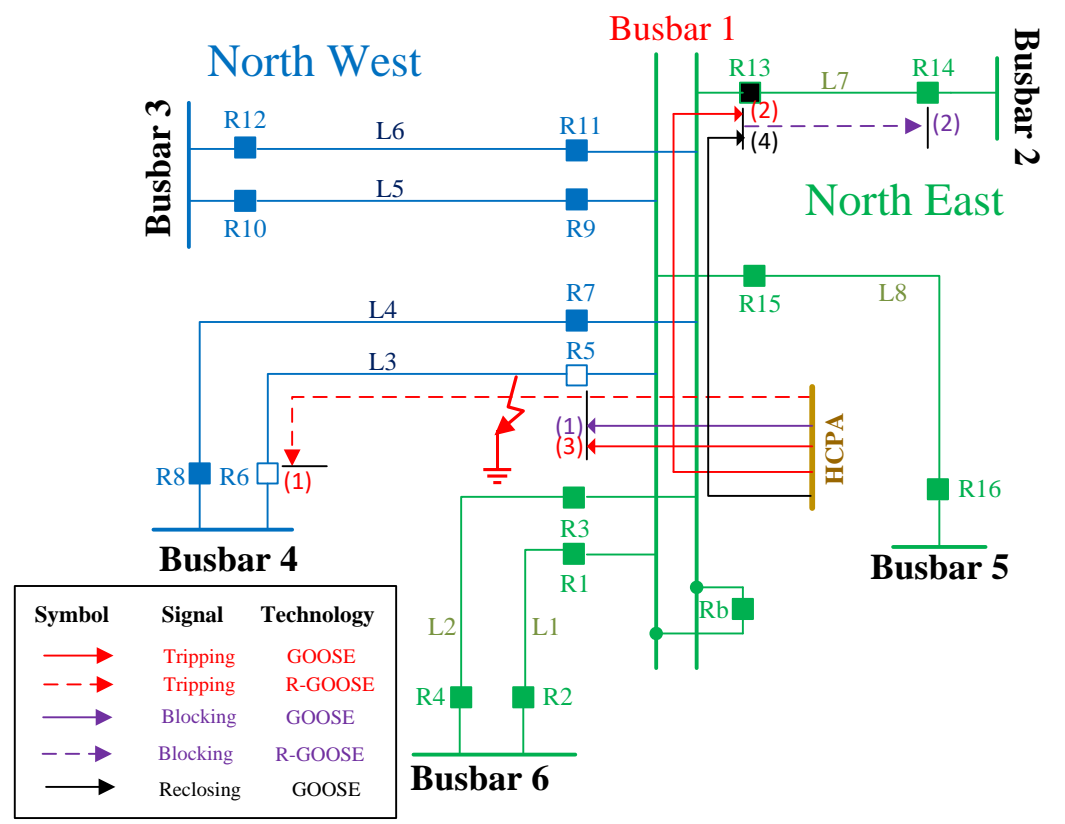

Figure 11.14: Quasi-dynamic simulation for Case 3.

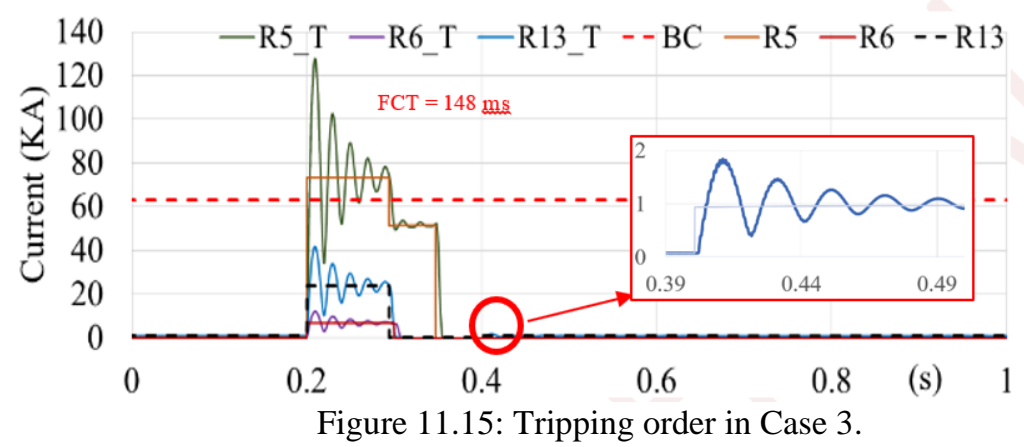


Table 11.3: The results of algorithm 1 for C3.

\begin{tabular}{|c|c|c|c|c|c|}
\hline Line & $I_{j}(\mathrm{kA})$ & $I_{S}=I_{f}-I_{k}^{m}(\mathrm{kA})$ & $I_{j}^{\prime \prime}(\mathrm{kA})$ & Candidate list & $R_{S} \& R_{S}^{\prime}$ \\
\hline L1 & 7.839 & \multirow{7}{*}{$73.655-63.00=10.31$} & -2.471 & \multirow{7}{*}{ L7 } & \multirow{7}{*}{$\mathrm{R} 13 \& \mathrm{R} 14$} \\
\hline L2 & 7.839 & & -2.471 & & \\
\hline L4 & 6.133 & & -4.177 & & \\
\hline L5 & 9.152 & & -1.158 & & \\
\hline L6 & 9.131 & & -1.179 & & \\
\hline L7 & 23.9 & & +13.59 & & \\
\hline L8 & 9.391 & & -0.919 & & \\
\hline
\end{tabular}

Table 11.4: The sequence of the events for $\mathrm{C} 3$.

\begin{tabular}{|c|c|c|c|c|c|c|}
\hline Command order & 1 & 2 & 3 & 4 & 5 & 6 \\
\hline Command & Block & Open & Open & Block & Open & Close \\
\hline Relay & $R_{k}=R_{5}$ & $R_{k}^{\prime}=R_{6}$ & $R_{S}=R_{13}$ & $R_{S}^{\prime}=R_{14}$ & $R_{k}=R_{5}$ & $R_{s}=R_{13}$ \\
\hline Max latency to receive signal from HCPA (ms) & 44 & 50 & 44 & 104 & 98 & 152 \\
\hline Circuit breaker & $C B_{k}$ & $C B_{k}^{\prime}$ & $C B_{s}$ & $C B_{S}^{\prime}$ & $C B_{k}$ & $C B_{S}$ \\
\hline Maximum operation time (ms) & - & 100 & 94 & - & 148 & 202 \\
\hline
\end{tabular}

\subsubsection{Case 3}

The fault and network condition in this case is exactly same as Case 2 but the protection scheme here is equipped with the developed HCPS to see the effectiveness of this method. Using the developed HCPS, the fault is cleared just by four switching actions as shown in Figure 11.14. Detailed description of these switching actions is as below.

Switching 1: By detecting the fault at zone 1 of R5 and activating the HCPA because of fault current magnitude, R5 is blocked and the tripping signal is sent to R6 immediately (command order \#1 and 2 in Table 11.4). Regarding the fault detection and classification (40 ms), R-GOOSE message delay (10 ms) and CB breaking time (50 ms), R6 operates after $100 \mathrm{~ms}$ of fault occurrence. It should be noted that although the R6 sees the fault in zone 2 and it would operate by time delay of zone 2 (additional $500 \mathrm{~ms}$ ), however, in the protection system and by detecting and tripping of a relay in one end of a transmission line, the trip signal is also sent to the relay on the other end of transmission line through the teleprotection system to ensure that the faulty part of the system is isolated in the minimum possible time. In this case, since the R5 cannot break the fault current, the HCPA sends this trip signal to the R6 to operate with minimum possible latency. 
Switching 2: While tripping signal is sent to R6, HCPS decides to break the current coming from L7 by sending tripping and blocking signals to R13 and R14, respectively (command order \# 3 and 4 in Table 11.4). L7 is selected by Algorithm1 presented in Figure 11.2. The detailed process of this algorithm is reported in Table 11.3. As can be seen in this Table, the HCPA receives all measured currents for all transmission lines connected to this busbar. To select a line for tripping, as mentioned in the Algorithm1, the HCPA calculates the surplus current (the current more than breaking capacity of the $\mathrm{CB}$ ) due to this fault $\left(I_{s}=10.31 \mathrm{kA}\right)$ and starts to create the candidate list by subtracting the measured current of each line from the surplus current. These values $\left(I_{j}^{\prime \prime}\right)$ are reported in column 4 of Table 11.3. The lines with positive value of $I_{j}^{\prime \prime}$ are added to the candidate list (In this case and as shown in Table 11.3, the only candidate is L7). The final candidate for tripping is the candidate with minimum value between all lines in the candidate list based on the Algorithm1 (in this case L7 is the final candidate). Therefore, R13 opens its CB in maximum $94 \mathrm{~ms}$ after fault beginning (40 ms fault detection and classification, $4 \mathrm{~ms}$ for GOOSE message and $50 \mathrm{~ms}$ for CB operation).

Switching 3: When SHC current coming from R13 is stopped, HCPA sends tripping command to R5 and the related CB opens its contacts $54 \mathrm{~ms}$ (4 ms delay for GOOSE message and $50 \mathrm{~ms}$ for breaking time) after operation of R13 to clear the fault from the system (command order \# 5 in Table 11.4). The maximum operating time for this CB is $148 \mathrm{~ms}$ as reported in Table 11.4.

Switching 4: By clearing the fault after $148 \mathrm{~ms}$ (FCT = $148 \mathrm{~ms}$ ), HCPA sends reclosing command to R13 to restore L7 to the service (command order \# 6 in Table 11.4). It takes maximum 4ms for GOOSE message to deliver to R13 and maximum $50 \mathrm{~ms}$ for reclosing. 
As a result, reclosing occurs in maximum $54 \mathrm{~ms}$ after fault clearance (In this case: $148+54=$ $202 \mathrm{~ms}$ after fault occurrence as reported in Table 11.4). Figure 11.15 shows RMS and transient values of currents for R5, R6 and R13. As it can be seen in this figure, R13 is reclosed at $\mathrm{t}=402 \mathrm{~ms}$ and carries its normal loading. Table 11.4 reports the sequence of the events and published signals by HCPA along with maximum latency for the operation.

The results demonstrate that developed protection scheme is highly successful to remove the SHC current from the system with a logical switching algorithm which uses data exchange capabilities defined in IEC 61850. This protection is comparable with ideal protection scheme in dealing with very high fault current in power system where we need to limit the fault current by installing expensive equipment like FCLs, in terms of fault clearing time and power system stability margin. It should be noted that the HCPA just isolated the faulty part of the system without using any FCLs and in fact, the developed HCPS just uses the capability of communication between protective relays using IEC 61850 standard. It is worth mentioning that less FCT will result in less rotor angle acceleration during the fault and as a result less overshot and more stability margin. Figure 11.16 shows the rotor angle of four generators located in four regions of this network for both case 2 (C2) and case $3(\mathrm{C} 3)$. As it can be observed from this figure, deploying the developed HCPS can significantly improve the stability margin of the system in presence of very high fault current in the system.

\subsubsection{Case 4}

This case demonstrates the performance of the protection system equipped with FCLs. In this case, the fault is seen in zone 1 by R5 and tripping will happen in maximum $90 \mathrm{~ms}$ while R6 is seeing the fault at zone 2. 

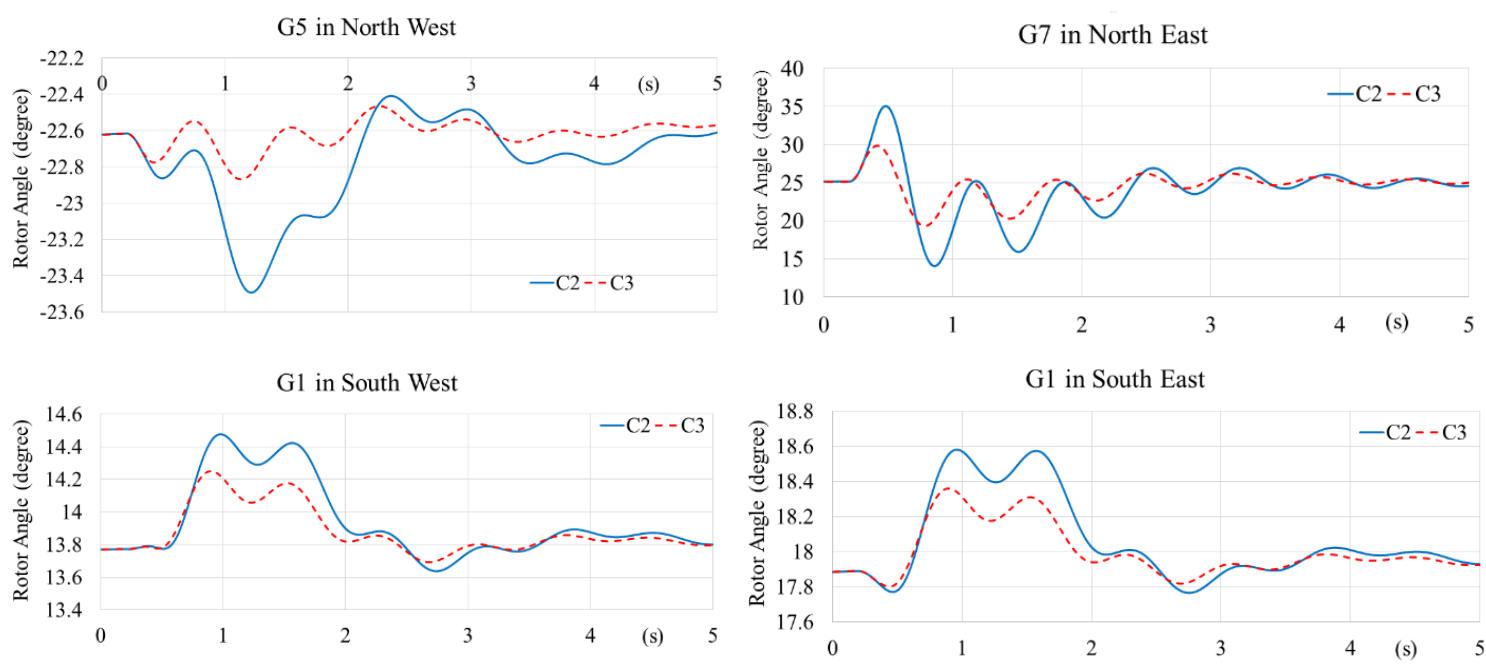

Figure 11.16: Rotor angle of four generators in the system for C2 and C3.

However, the tele-protection is used by R5 to send the trip signal to R6. As mentioned earlier, the maximum latency for this signal is $10 \mathrm{~ms}$, therefore, R6 is operating in maximum $100 \mathrm{~ms}$. In this case, like case 1, the fault is cleared by two switching actions and just the faulty line is isolated from the rest of the system, but we need to install an expensive FCL in the system which needs high maintenances.

\subsubsection{Case 5}

To show the flexibility of developed HCPS regarding the network conditions and equipment, in this case we consider the same conditions as reported in Case 2 but instead of using a $\mathrm{CB}$ with $40 \mathrm{kA}$ as breaking capacity at busbar coupling position, we assume the $\mathrm{BC}$ of this circuit breaker is $50 \mathrm{kA}$. It means that this $\mathrm{CB}$ can break the fault current while the CB located in R5 is unable to break it. In this case, and as shown in Figure 11.17, by occurring the fault at $200 \mathrm{~ms}$ in this simulation, R5 detects the fault in zone 1 and HCPA compares the fault current with $\mathrm{BC}$ of circuit breaker and blocks the $\mathrm{R} 5$ while sends tripping signal to R6. Measurements show that Rb is passing $46.995 \mathrm{kA}$ which if it is broken, the fault current through R5 $(29.11 \mathrm{kA})$ would be much less than its BC (63 kA). 


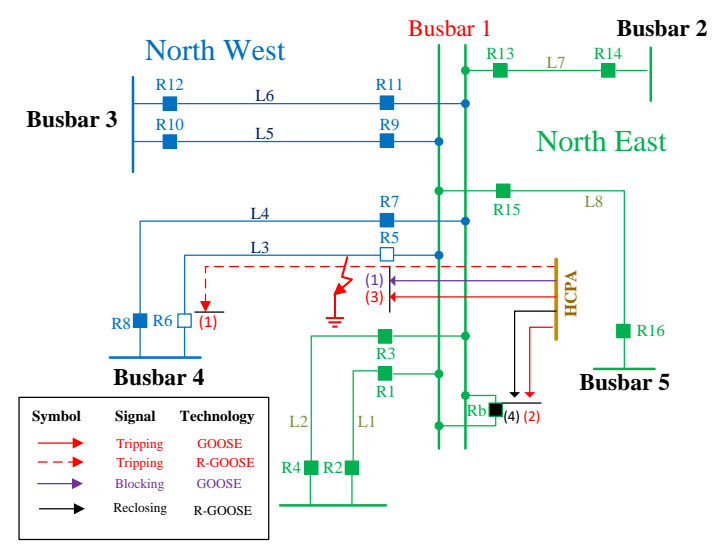

Figure 11.17: System status in Case 5.

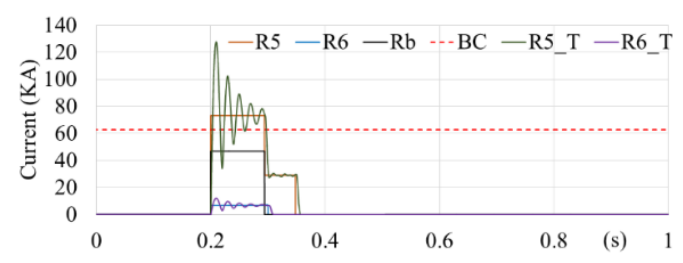

Figure 11.18: Simulation results for Case 5.

Therefore, HCPA sends the tripping signal to $\mathrm{Rb}$ (maximum $4 \mathrm{~ms}$ for this GOOSE message) after detecting the fault at zone 1 (considering $40 \mathrm{~ms}$ for fault detection and classification) and braking of $\mathrm{CB}$ at this position (maximum $50 \mathrm{~ms}$ ). Therefore, $\mathrm{Rb}$ trips its $\mathrm{CB}$ in $94 \mathrm{~ms}$ after the fault occurrence. Following this trip and by decreasing the fault current at R5, HCPA sends the tripping command to R5 (maximum $4 \mathrm{~ms}$ ) and waits for CB opening (maximum $50 \mathrm{~ms}$ ).

As shown in Figure 11.18, the fault is cleared $54 \mathrm{~ms}$ after $\mathrm{Rb}$ tripping which means that FCT for this case is $148 \mathrm{~ms}$ after fault start. It is clear that after tripping of R5, Rb can be reclosed quickly. The tripping orders and quasi-dynamic simulation for this case are shown in Figures 11.17 and 11.8, respectively. The summary of these protection actions is 4 switching actions and one transmission line outage with $148 \mathrm{~ms}$ as FCT. Table 11.5 summaries the protection results for all study cases. As can be seen in this Table, although the best results are from Case 4 (system equipped with FCL), the developed HCPS in Case 3 and Case 5 have also acceptable performance in dealing with very high fault current. The FCT for both C3 and C5 is maximum $148 \mathrm{~ms}$, which is just $48 \mathrm{~ms}$ more than FCT in C4 (100 ms), and much less than $\mathrm{C} 2$ where the conventional distance protection is operated by 
Table 11.5: The protection results for $\mathrm{C} 1$ to $\mathrm{C} 5$.

\begin{tabular}{|c|c|c|c|c|c|c|c|}
\hline Case & $\begin{array}{c}\text { SHC in R5 } \\
(\mathrm{kA})\end{array}$ & FCL installation & Protection scheme & $\begin{array}{c}\text { Maximum } \\
\text { FCT }(\mathrm{ms})\end{array}$ & $\begin{array}{c}\text { Maximum rotor } \\
\text { angle of G7 }\left(^{\circ}\right)\end{array}$ & $\begin{array}{c}\text { Number of } \\
\text { Switching actions }\end{array}$ & $\begin{array}{c}\text { Number of } \\
\text { tripped lines }\end{array}$ \\
\hline Case 1 & 12.375 & No & Conventional Distance & 90 & 27 & 2 & 1 \\
\hline Case 2 & 73.655 & No & Conventional Distance & 490 & 35 & 8 \\
\hline Case 3 & 73.655 & No & HCPS & 148 & 30 & 4 \\
\hline Case 4 & 48.144 & Yes & Conventional Distance & 100 & 28 & 1 \\
\hline Case 5 & 73.655 & No & HCPS & 148 & 30 & 2 & 4 \\
\hline
\end{tabular}

delay of zone 2. It is obvious clear that the number of switching actions by developed HCPS is twice the number of actions in $\mathrm{C} 4$, but the results in term of number of tripped lines is the same for both cases. In fact, the developed HCPS uses the communication capabilities defined in IEC 61850 to define switching priorities in such a way that the main CB can break the fault current, which is less than its $\mathrm{BC}$ due to tripping other transmission lines, which are feeding the fault.

\subsection{Summary}

In this chapter, a complementary protection scheme was developed to equip the protection system with an intelligent switching algorithm to deal with very high short circuit currents which are higher than breaking capacity of existing circuit breakers. In this protection scheme, the distance protection system is still responsible for clearing faults form the transmission system and the developed HCPS is just activated if fault current is more than breaking capacity of circuit breakers in the system. The communication protocols defined in IEC 61850 are deployed by a supervisory protection agent where it publishes control commands to protective relays for cascading switching in the system. The numerical results verified the effectiveness of this method and they were compared to results from ideal case, which uses an FCL to reduce the fault current, and results from conventional distance protection scheme. It was shown that the results form developed HCPS is very close to ideal case while very high FLC installation and maintenance costs are not needed anymore. In addition, the developed HCPS does not need any network 
reconfiguration or service interruption for implementation because this protection layer is added to the system by connecting an IED to the network switch in LAN where are protective relays in the substation are connected. Although the developed HCPS in this dissertation is developed for $\mathrm{HV}$ transmission system, in future works, the application of this protection scheme for distribution network with high penetration of inverter-based DGs could be investigated to remove the necessity of installing FCL in the distribution network. 


\section{Chapter 12 Conclusions and Recommendations for Future Work}

In this dissertation, we developed an effort relating to electricity market design and optimization for secure energy transactions in a multi-microgrid system. Optimization techniques and ancillary services in the power market were developed and verified. Furthermore, the concept of critical communication in the modern power system, along with its security issues and applications were studied to provide a practical level of security aware microgrid infrastructure. Here we provide concluding remarks and provide some recommendations for future works are suggested.

The concept of electricity market design for modern power systems contains several technical and regulatory challenges. It is because of the new structure of modern power systems with high penetration of RESs, DGs, EVs, and autonomous MGs, where all of them want to participate in open-access electricity markets to trade electric energy actively. Before developing an optimal and secure electricity market framework for these new market players, which usually are in distribution networks, different aspects of electric markets should be evaluated. For example, the robustness of electricity market platform against cyber-attacks, the structure of the market in terms of market-clearing price, the level of competition and effectiveness of the market to address the quick changes in demand and supply, communication infrastructure and its security issues are some challenges in this area.

In this dissertation, the centralized, decentralized, and distributed structures of the electricity market, as three main market platforms, were studied from the cybersecurity point of view and robustness against external cyber-attacks. The results demonstrated that the distributed market platform is more secure than the other two market structures. 
Furthermore, it was shown that the decentralized market has a better performance comparing to the centralized energy transaction framework in dealing with external cyberattacks. Therefore, decentralized and distributed market frameworks were chosen to develop multi-agent-based electricity markets for multi-microgrid systems.

At first, an optimal multiagent-based market algorithm for smart multi-microgrid systems was developed. In that market, a game-theoretic double auction mechanism was introduced for the day-ahead market; a hierarchical optimization algorithm was developed for the hour ahead market and a real-time market inter-microgrid market. Results demonstrated that using this market platforms; the autonomous microgrids could optimally use the capabilities of all agents to decrease the energy cost, the dependency of the area on the utility grid and optimization solution time.

Afterward, the data exchange among market agents for the developed decentralized market structure was studied, and a data-centric communication framework was developed and deployed based on the DDS standard. Then this platform was modified to decrease the number of topics over the DDS domains through an effective ID management scheme, and the DDS security algorithm was deployed to ensure the data security in this framework by defining user authentication, key management, and access permission control schemes.

Since the distributed market platform is inherently highly secure, we tried to develop a distributed electric market for multi-microgrid systems considering technical challenges in power systems. Therefore, we developed a hierarchical blockchain-based (HBC) energy transaction framework for inter-microgrid energy trades in a multi-microgrid system. In the developed HBC electricity market, we developed and deployed an equivalent model for the power system to simulate the energy transactions and run the power flow 
calculations before approving them to ensure the secure operation of the power system. Numerical results confirmed the effectiveness of the developed HBC algorithm for intermicrogrid energy transactions, participant privacy improvement and reducing the ledger size for higher-level blockchains in the system.

After developing electricity market frameworks, we worked on two ancillary services in power markets; Energy imbalance ancillary service and real-time congestion management. For the first one, we developed an OPR scheme between and within ICs to minimize the power imbalance factor at the PCC, active power losses and voltage deviation indices in the grid-connected mode of an unbalanced hybrid AC-DC microgrid. The effectiveness of the developed algorithm was confirmed through numerical results obtained from the simulation of the modified IEEE 13 bus system as a highly unbalanced hybrid AC-DC microgrid and IEEE 34-bus test system as an unbalanced distribution system. Furthermore, to show the effect of the power routing between charging stations on the active power losses reduction, a laboratory-based smart microgrid was established and examined. Numerical results demonstrated that the developed OPR scheme was highly successful in minimizing the objective functions and smoothing the voltage profile within the system. Consequently, optimal operation of unbalanced hybrid AC-DC microgrids would be possible when the developed OPR algorithm is implemented.

For the second one, we developed an adaptive real-time congestion management algorithm considering the adaptive thermal rating of transmission lines, which uses the thermal adaption of the conductors in two steps during the congestion management process. Dividing the congestion removal progress into the two phases, incorporating the FACTS devices capability and DR in the optimization problem, made it possible to find feasible 
and considerably cost-effective solutions for the RTCM problem. Furthermore, a hybrid real-time optimization framework was developed based on the AANN and the modified PSO algorithm, which is considered as a highly accurate and robust optimization tool for the RTCM problem. Numerical results illustrated that the developed algorithm is costeffective, highly secure and reliable in critical cases when the one-step RTCM method fails to manage the congestion properly.

Another aspect of this dissertation was about critical communications in modern power systems, where advanced communication protocols are utilized. Therefore, we developed a multicast communication framework for R-GOOSE messages over the WAN. The skeleton of this framework is based on a data-centric communication approach to address the technical requirements for time-sensitive and critical communications in smart power systems. This approach encapsulates the GOOSE data model into the DDS data object and routes it over the network to its final destinations. The effectiveness of the developed framework was validated by the experimental results by measuring the latency of this framework, showing that this approach only took around $20 \%$ of the maximum defined time for IEC 61850 routable communication and gave us around $8 \mathrm{~ms}$ to manage the network traffic. Furthermore, the best effort of the QoS profile along with the proper message priority policy could guarantee the message delivery within its defined time span.

In the next step, we studied the security of the developed framework for R-GOOSE messages to address the security issue of critical time-sensitive routable communications in the smart grid. For this aim, at first, the DDS security algorithm was deployed to the MRGM framework, and its latency was measured using an experimental setup. Afterward, a bad data injection cyber-attack was lunched on this framework to compare the MRGM 
framework without DDS security measure with the one with the DDS security algorithm. The results showed that the DDS security algorithm is fast and secure enough for this type of communication in power systems. The second security approach was introduced for routable communication in power systems based on a sequence hopping algorithm, which was called Secure Sequence Hopping Algorithm. This security method is independent of the communication middleware and is very fast and reliable for data integrity and security of critical communications in smart grids.

Finally, we studied the application of the secure routable communication framework in a modern power system by proposing a complementary protection scheme. This scheme is used to equip the protection system with an intelligent switching algorithm to deal with very high short circuit currents, which are higher than the breaking capacity of existing circuit breakers. In this protection scheme, the distance protection system is still responsible for clearing faults from the transmission system, and the developed HCPS is just activated if the fault current is more than breaking capacity of circuit breakers in the system.

The communication protocols defined in IEC 61850 are deployed by a supervisory protection agent where it publishes control commands to protective relays for cascading switching in the system. The numerical results verified the effectiveness of this method, and they were compared to results from the ideal case, which uses an FCL to reduce the fault current, and results from conventional distance protection scheme. It was shown that the results form developed HCPS are very close to the ideal case, while very high FLC installation and maintenance costs are not needed anymore. In addition, the developed HCPS does not require any power network reconfiguration or service interruption for 
implementation because this protective layer is added to the system by connecting an IED to the network switch in LAN where are protective relays in the substation are connected.

\subsection{Recommendations for Future Work}

The multi-microgrid electricity markets developed and developed in this dissertation address the challenge of designing the optimal and secure electricity markets for a new structure of modern power systems. Several technical and regulatory difficulties were covered in this work, including minimum dependency of a multi-microgrid area on the external utility grid, fast market clearing process, and reliability and security of competitive market structures. Furthermore, a new hierarchical blockchain-based electricity market was developed and developed for a multi-microgrid system to address the transaction feasibility assessment in distributed markets. However, electricity market architectures are always complicated because they need to cover both market issues and power system technical challenges. Therefore, it is a multidisciplinary research field with many research opportunities. Following we recommend some future research topics in this field:

- Competitive blockchain-based electricity markets: Developing an auction-based and competitive electricity market using the blockchain platform would be in an ingesting topic, which can address participants' competition in an auction to decrease the overall energy cost ultimately.

- Improving the privacy of market players: The ledger transparency is a unique feature of blockchain technology; however, it is in contrast with participants' privacy. Therefore, improving the customer's privacy within the blockchain platform is a possible research opportunity.

- The potential of joining mobile loads to private local blockchains: Since the EVs 
are considered as mobile loads in the power system, they can connect to different microgrids and consequently, their private local blockchains. Therefore, a proper strategy is needed to address this issue.

- Expanding HBC technology to cover energy transactions in bulk power systems: We developed the HBC for a multi-microgrid system within a distribution network. However, this technology can be used for bulk power system transactions over the high voltage transmission systems. Developing proper equivalent models for largescale meshed networks for energy transaction evaluation is another research potential for future works. 


\section{Appendices}

\section{Appendix I: Game theoretic-based Double auction mechanism}

\section{1- Agent development}

In order to develop agents for LA, DA and GA in DIgSILENT PowerFactory, the DPL language is used to collect data from Loads, DESSs and DG within each MGs. Regardless of power system software, which is used to model the system, these data can be collected and categorized into $\mathrm{C} 1, \mathrm{C} 2$ and $\mathrm{C} 3$ as mentioned in this market algorithm. We exported the data to text files by specific format shown in Figure 1, and set data to Excel files, Figure 2 , to run the double auction mechanism through a MATLAB script as shown in section 2 .

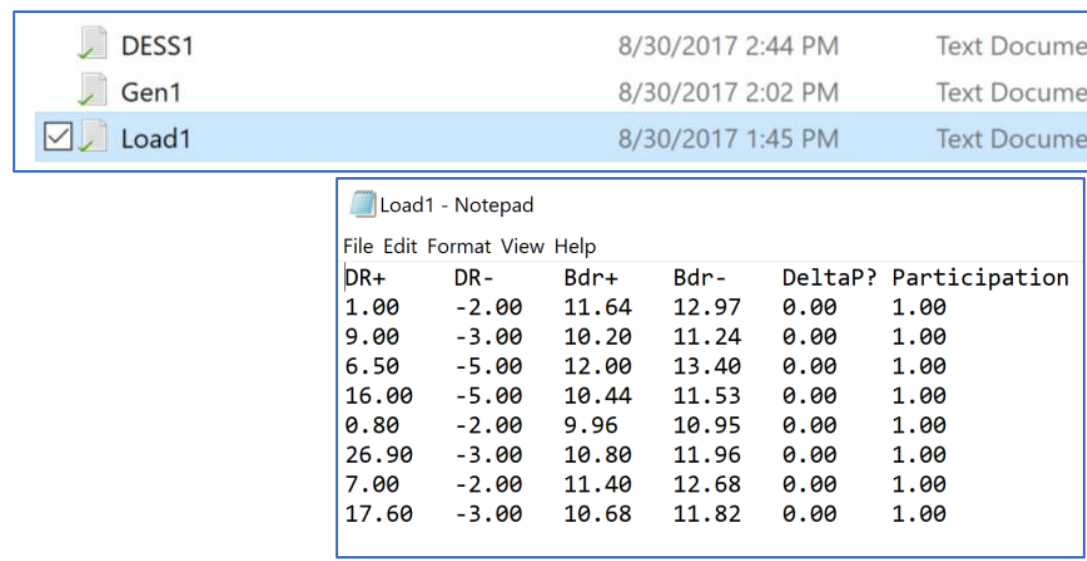

Figure 1: Load agent output form DigSilent PowerFactory

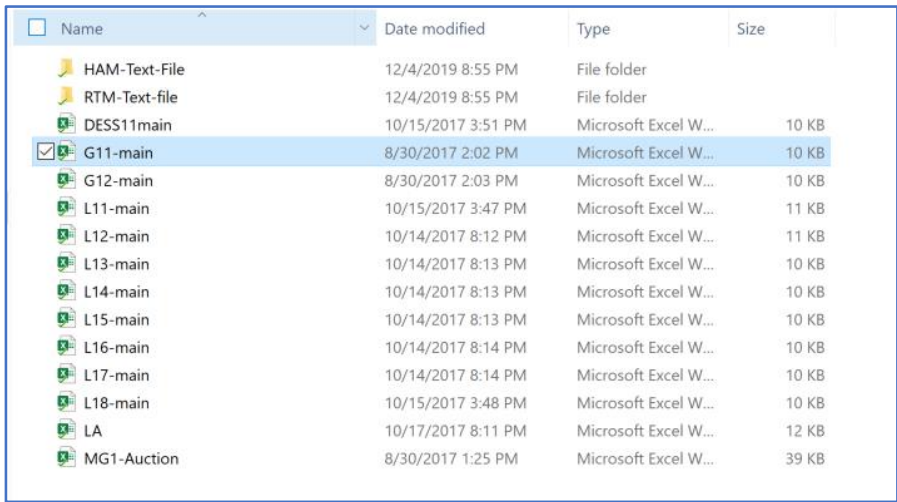




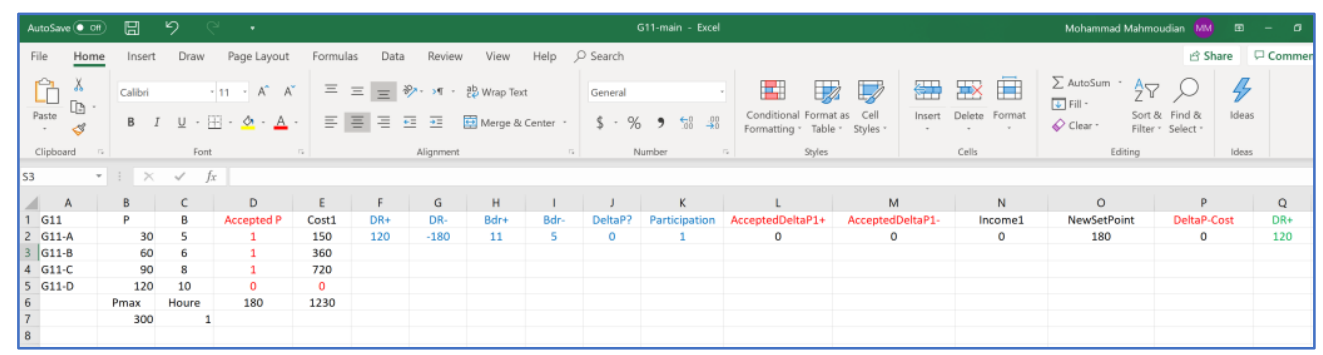

Figure 2: Double auction data set

2- Double auction development

This section contains a MATLAB code which can clear the double auction mechanism,

determines the MCP and winners and loser in the market. The results are returned to text file where the agents can read them, change their bid values and resend for another round of the market. The following code is used for all rounds of the market and save data in order which are received.

\section{MATLAB Codes:}

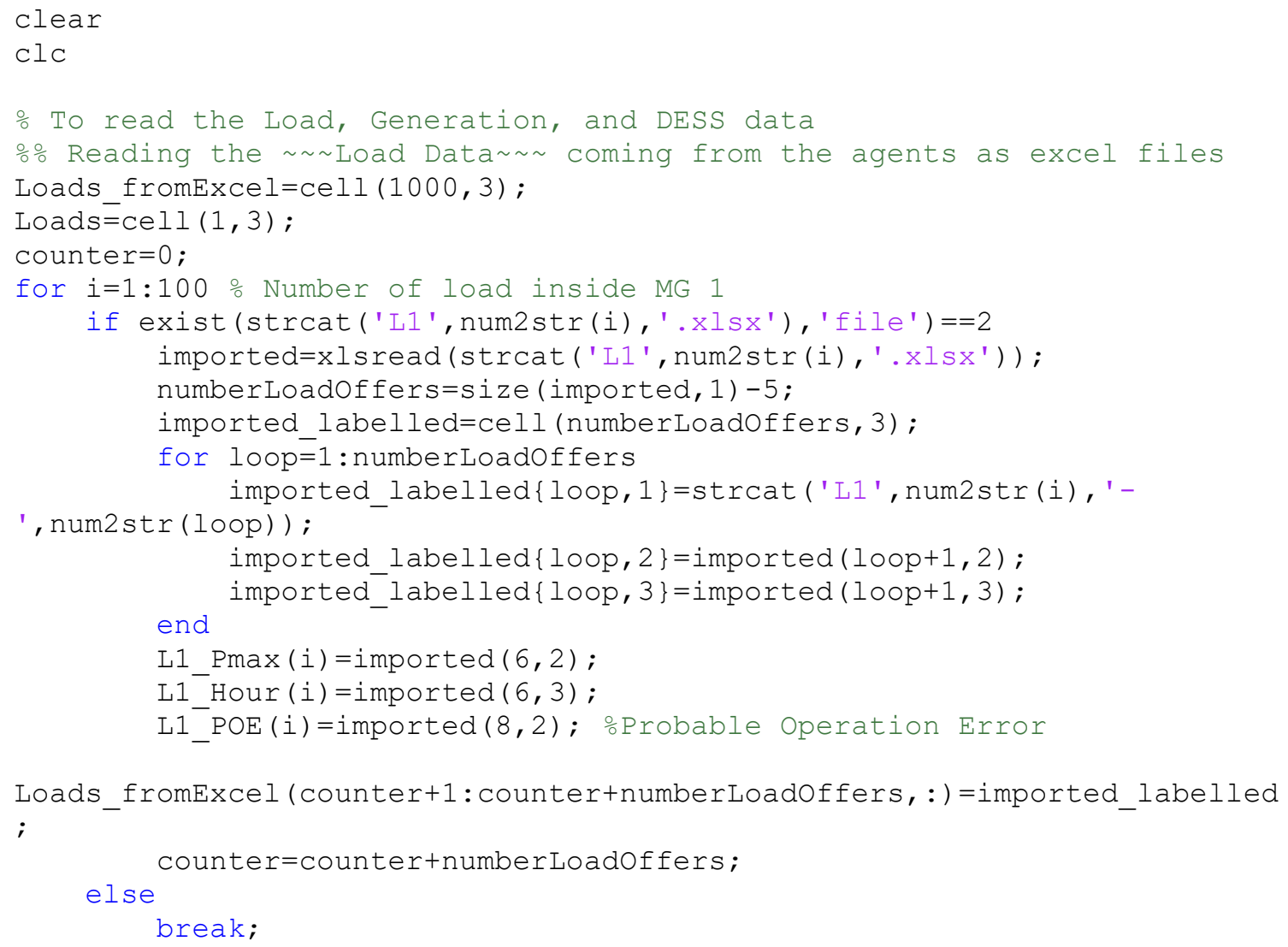




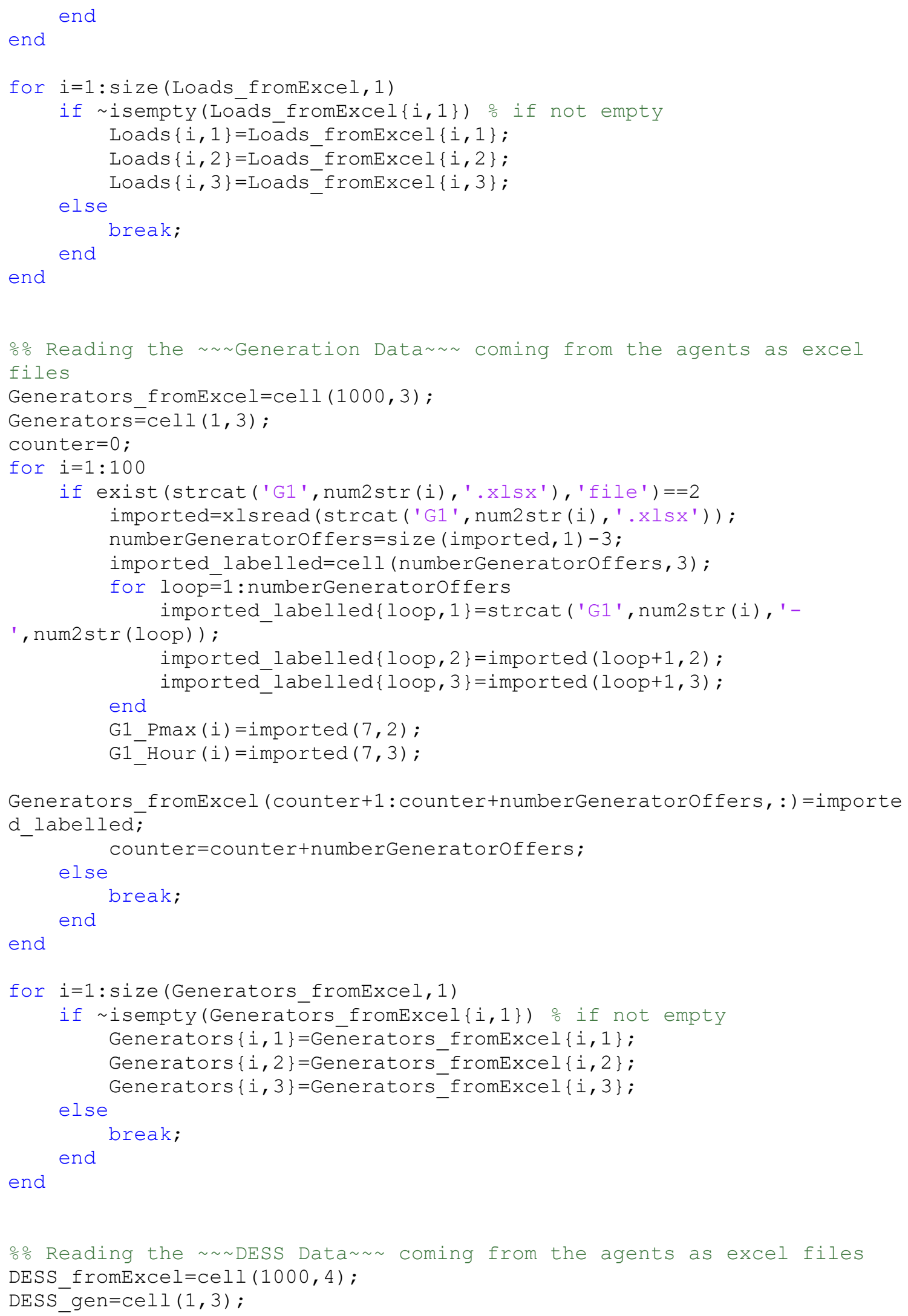




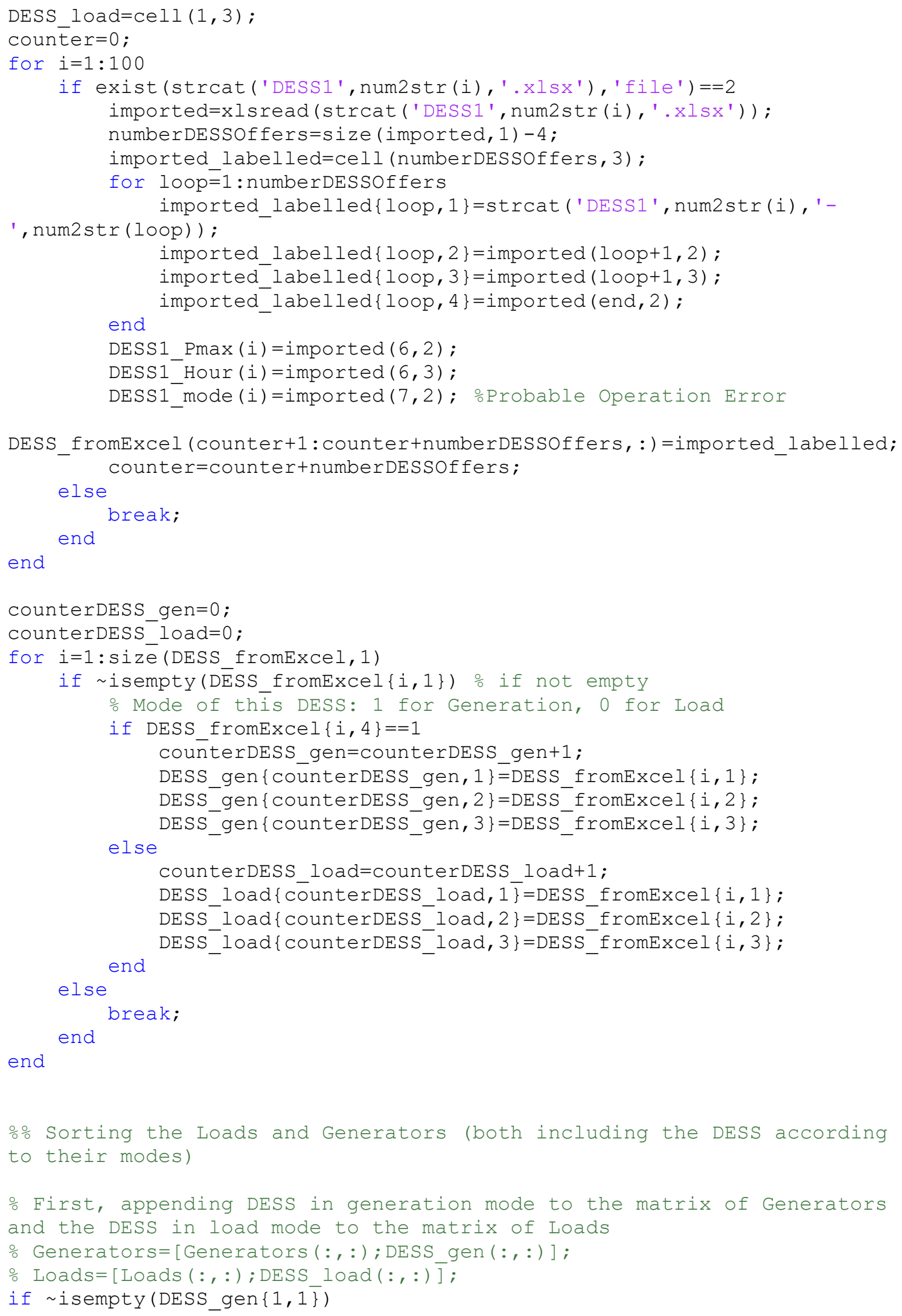




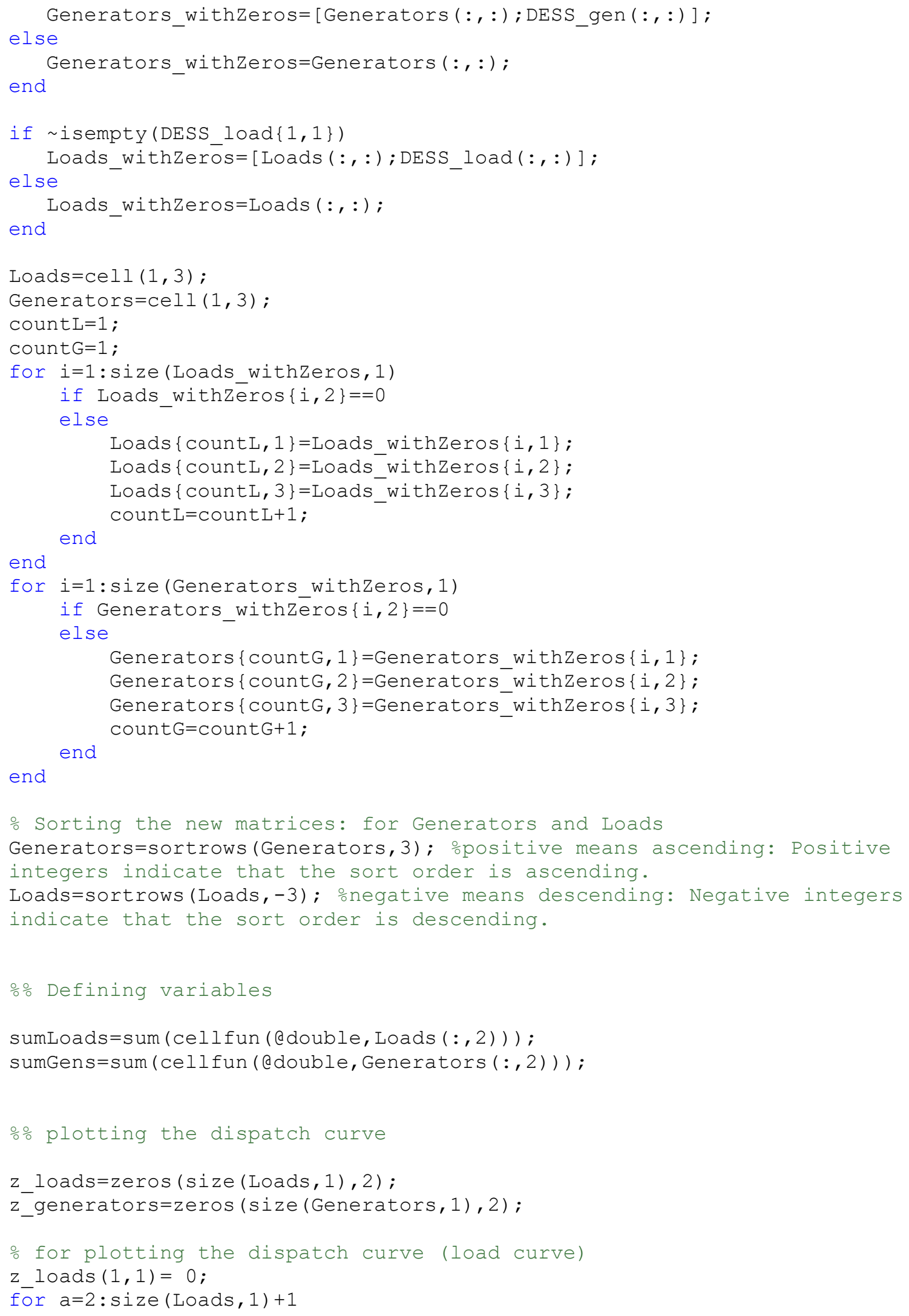




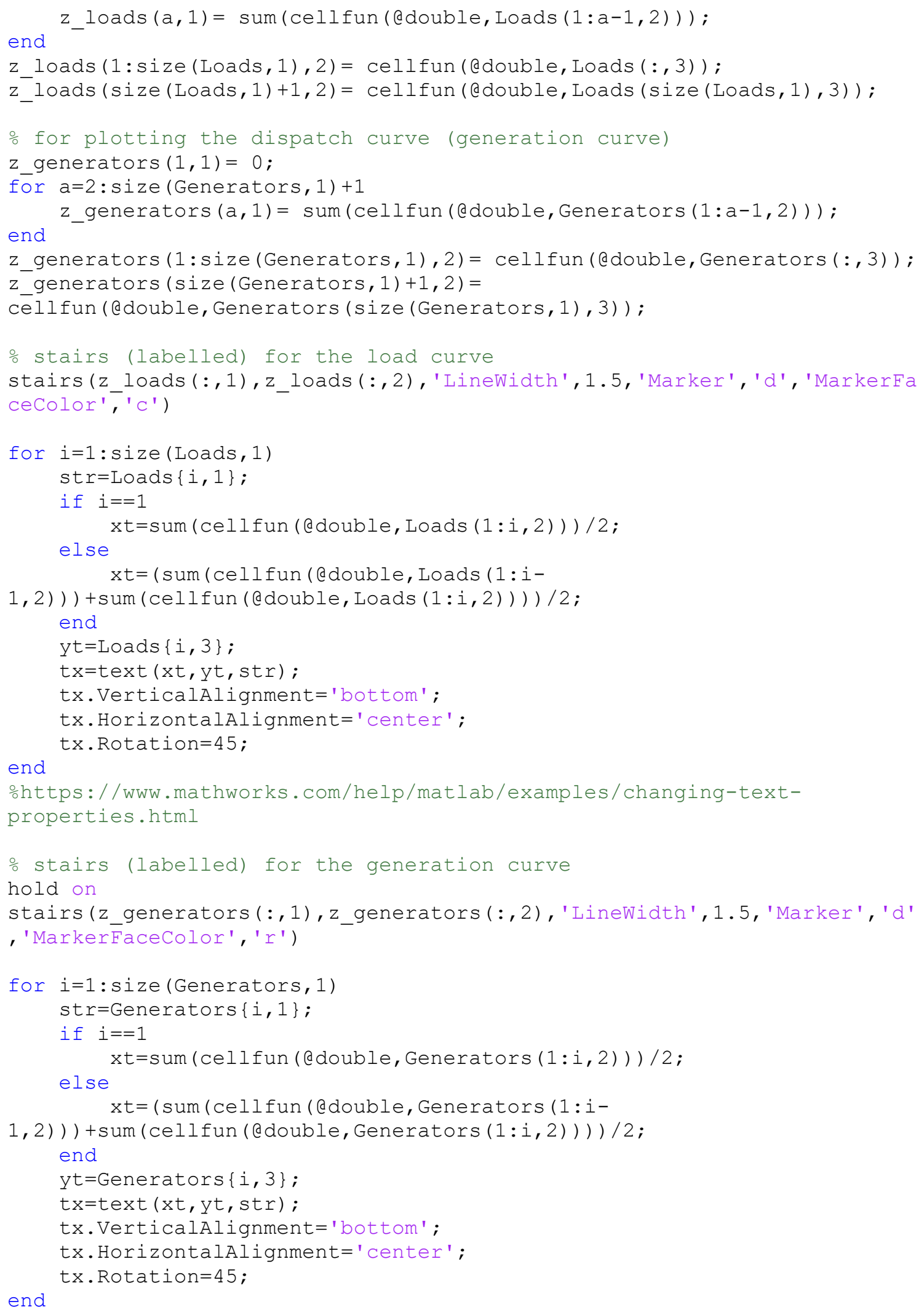




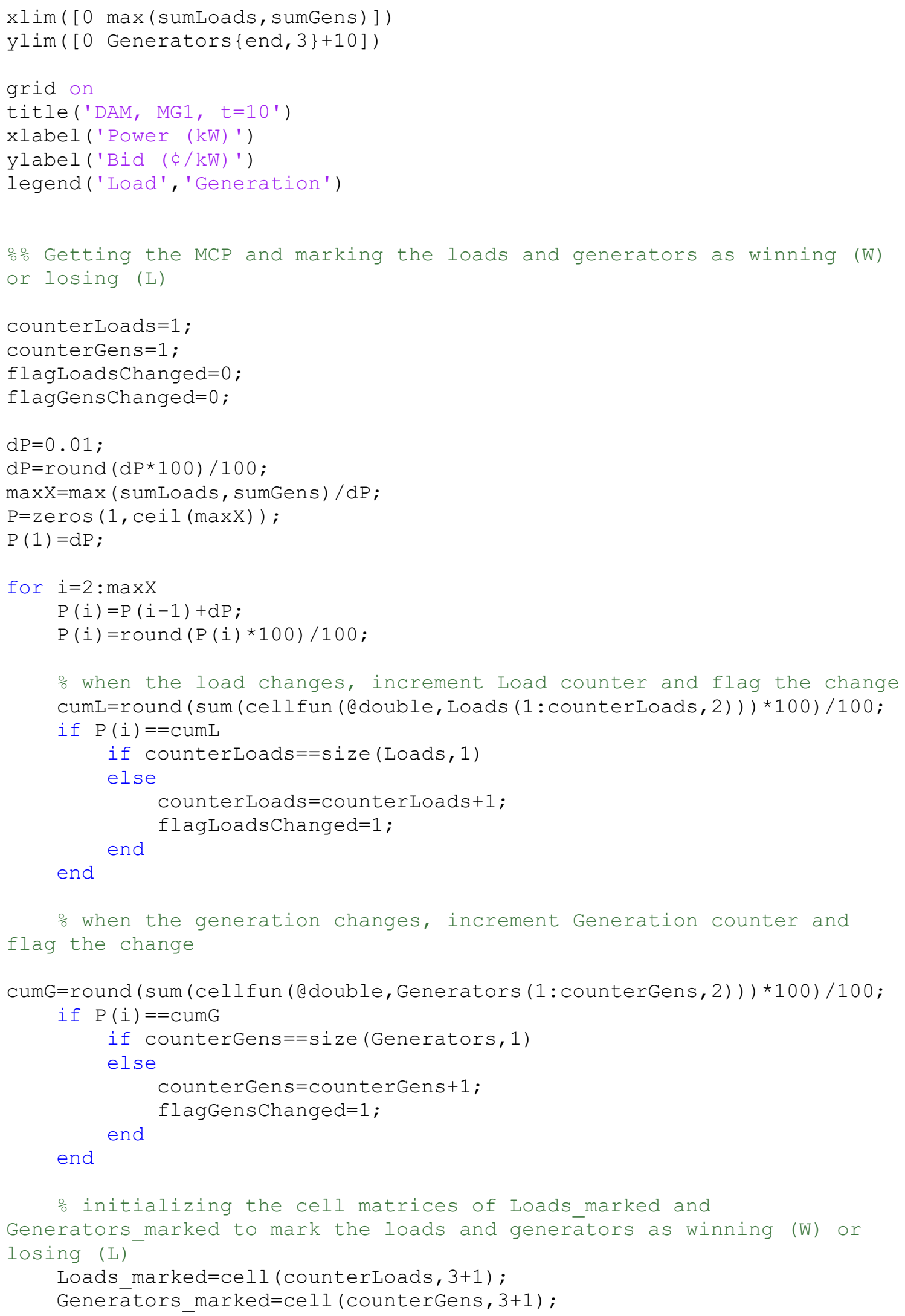




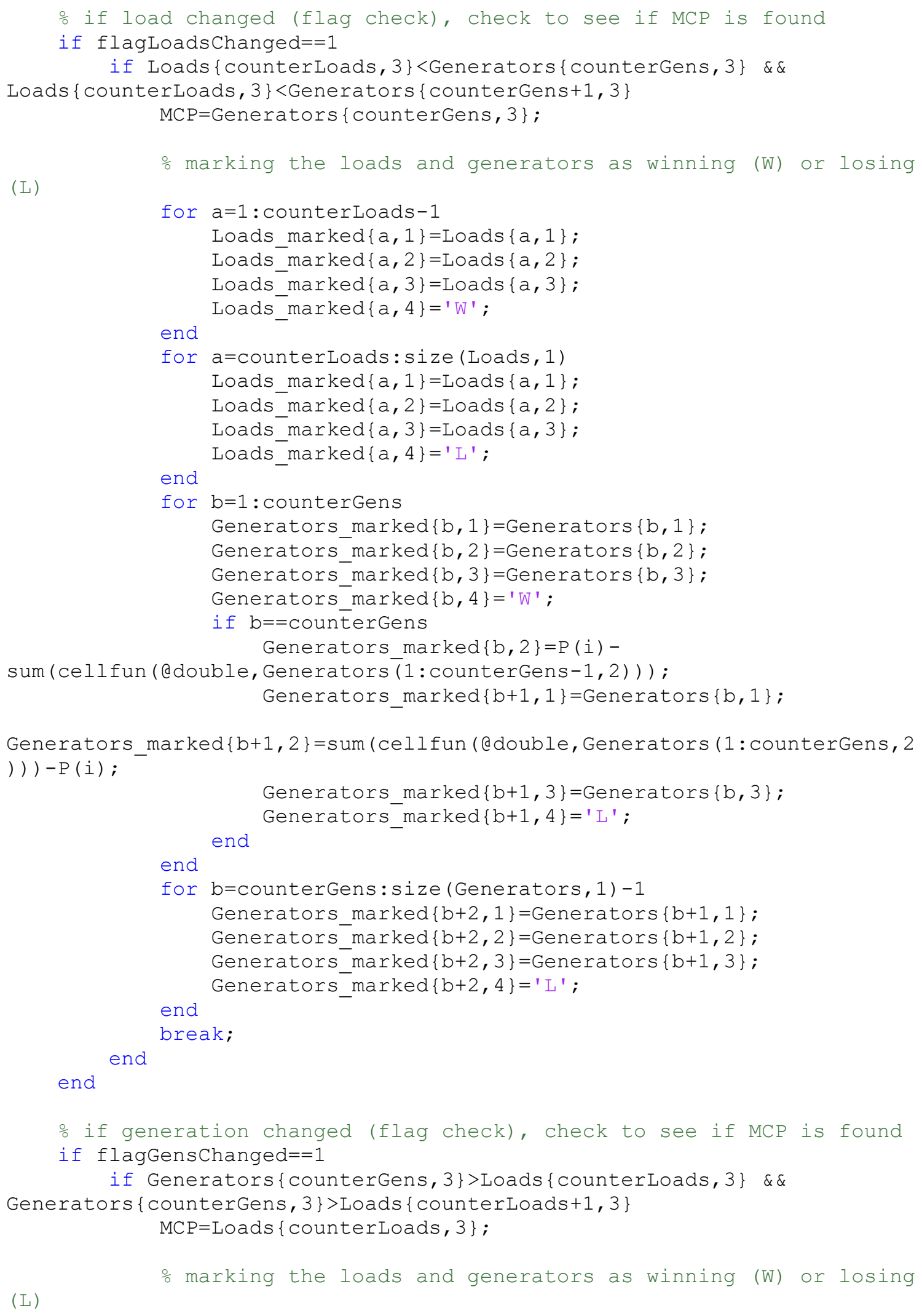




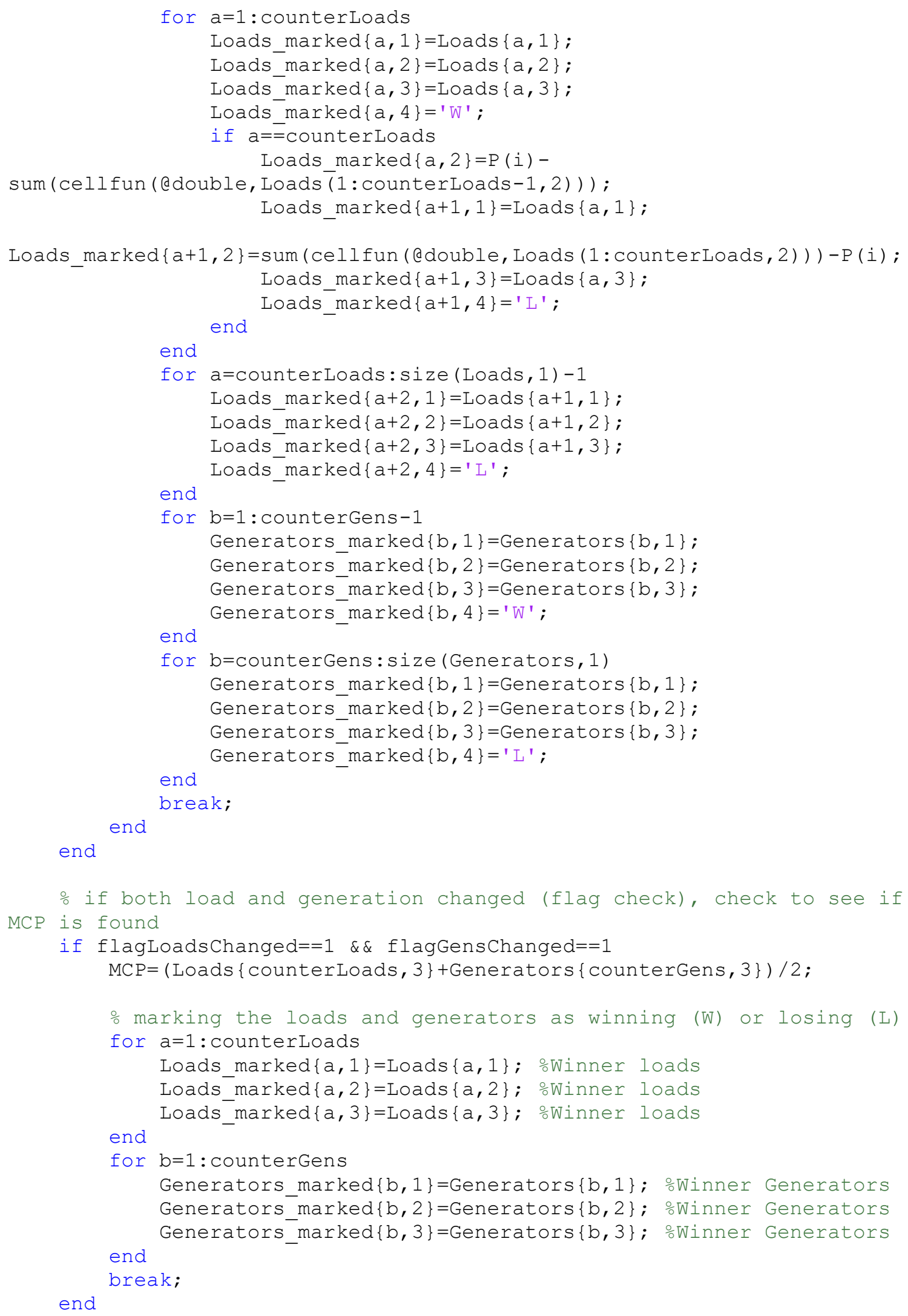


\% reset flags to 0

flagLoadsChanged=0;

flagGensChanged=0 ;

end

\% A step for arranging the generators and loads back into separate excel files, but now marked as winning or losing

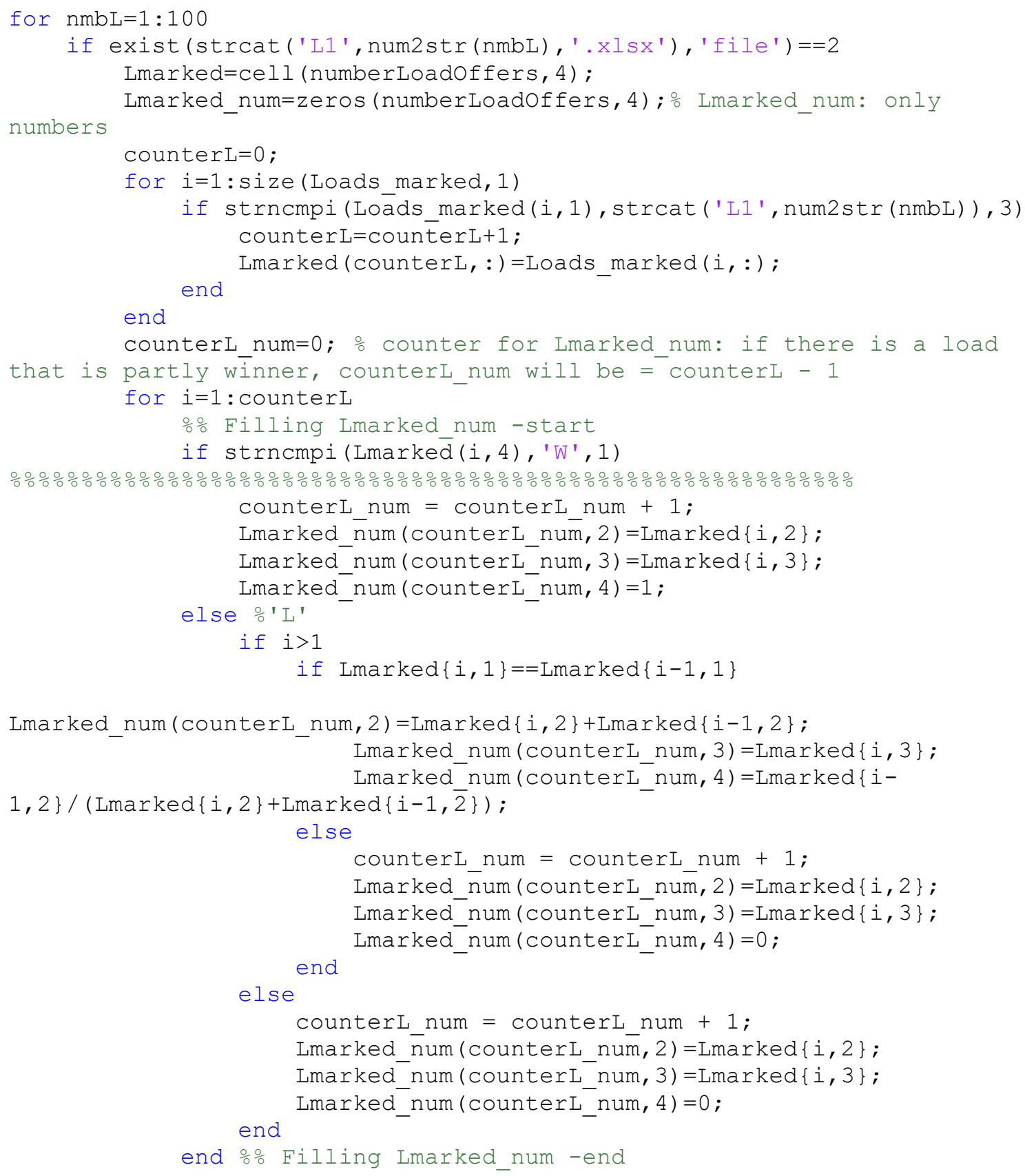




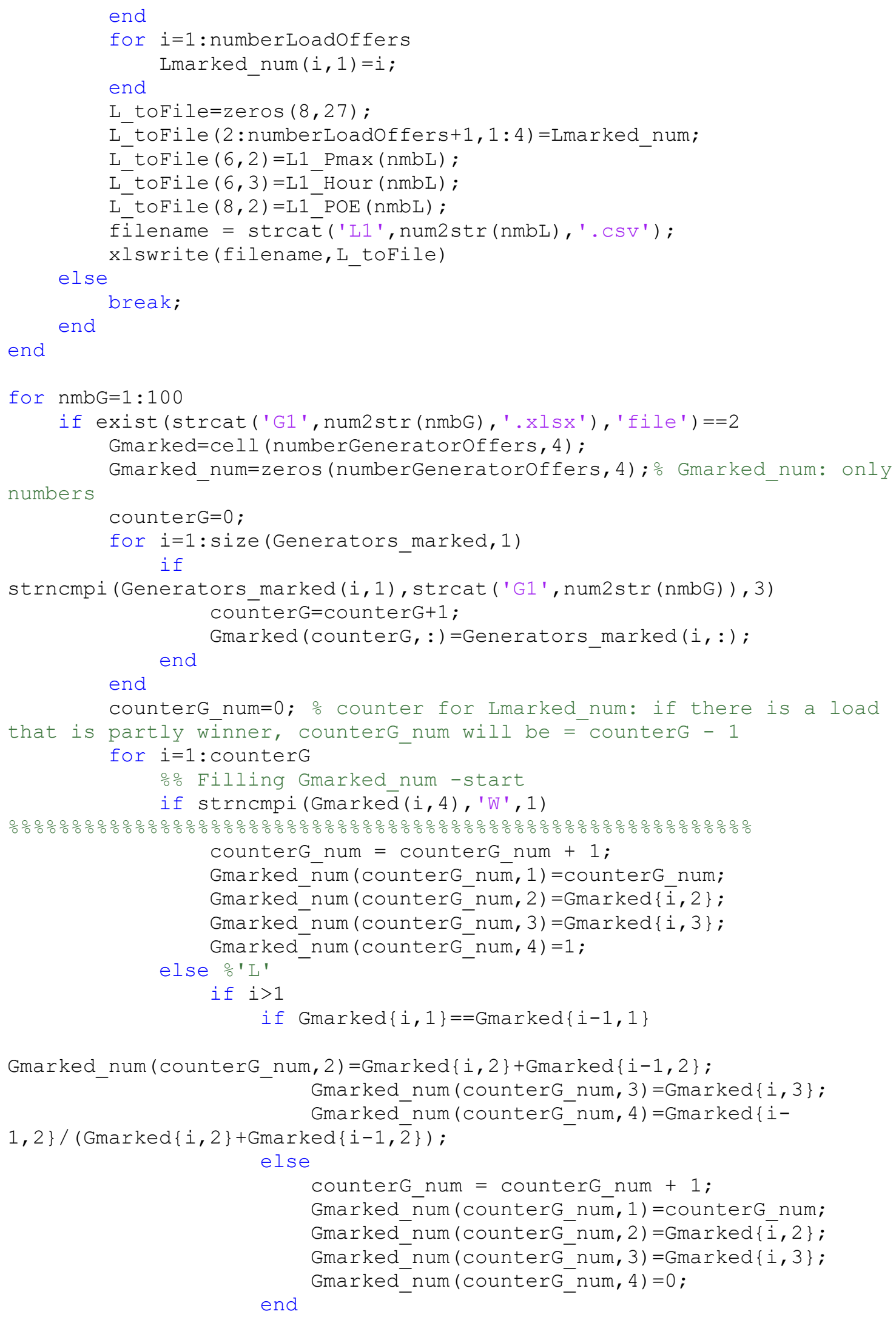




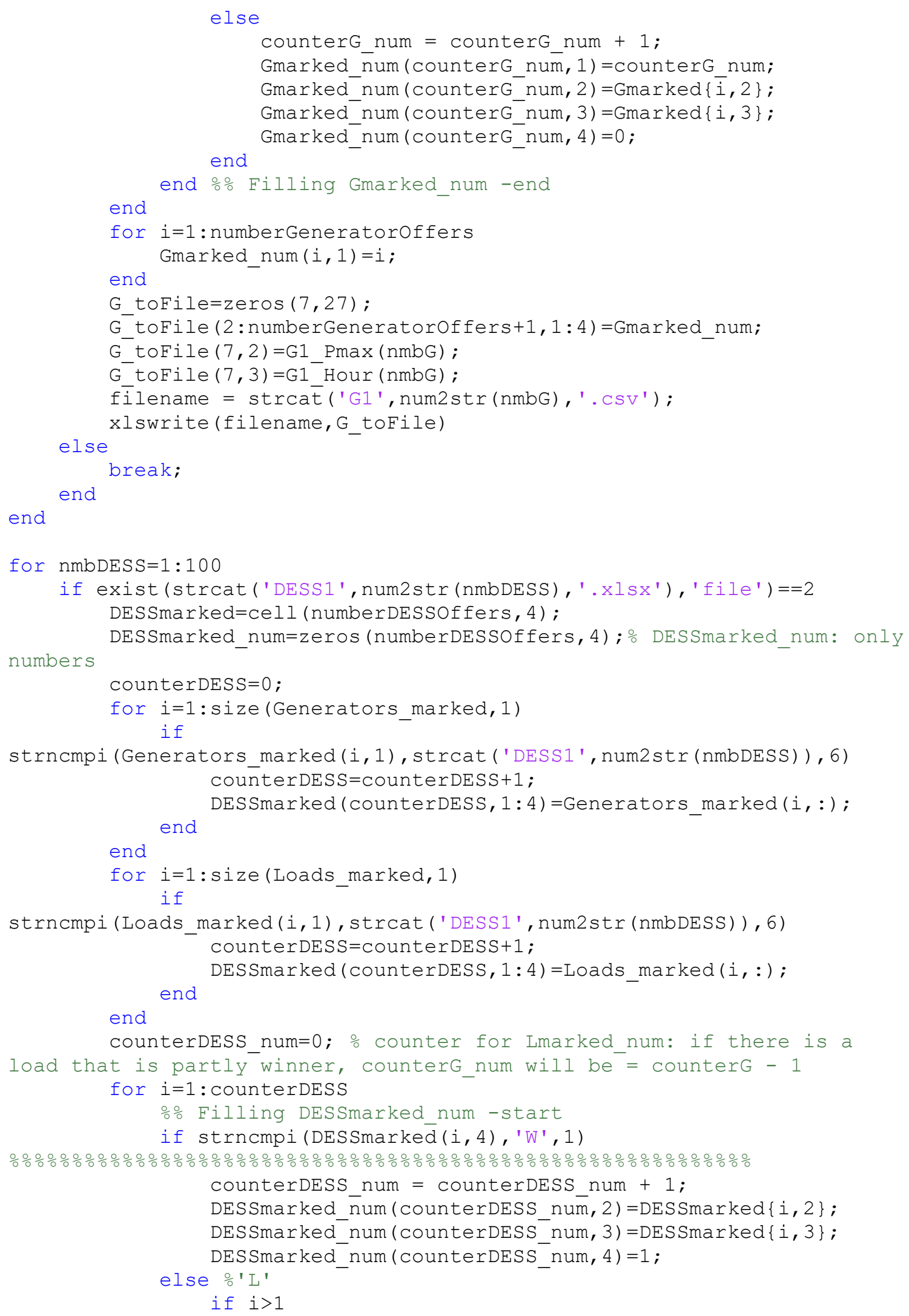




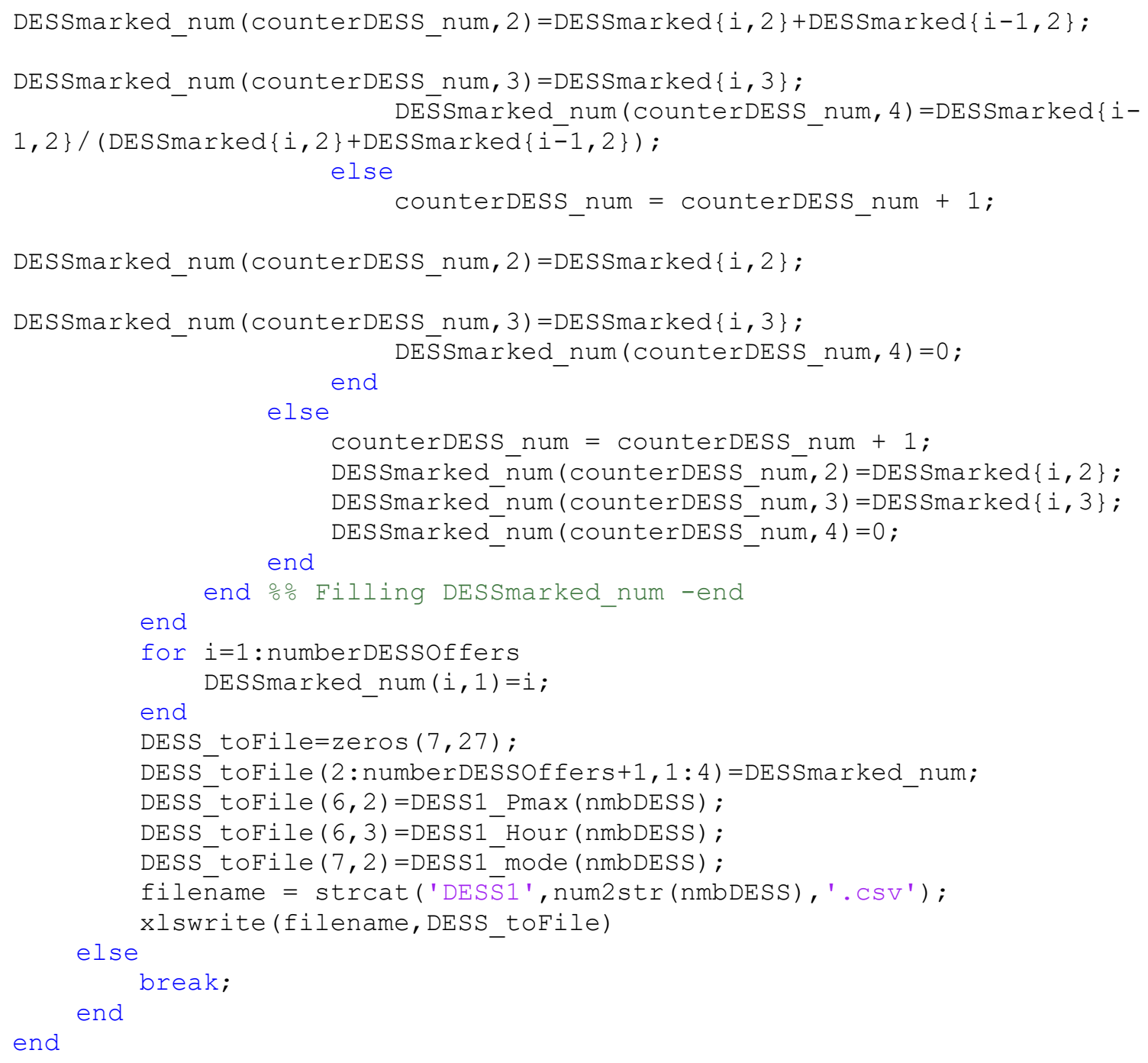

The results are shown in Figure 3 for five rounds of this double auction based on the biddings of market players. Detailed results have been reported in Table 4.1: DAM results for 9th and 13th intervals. 


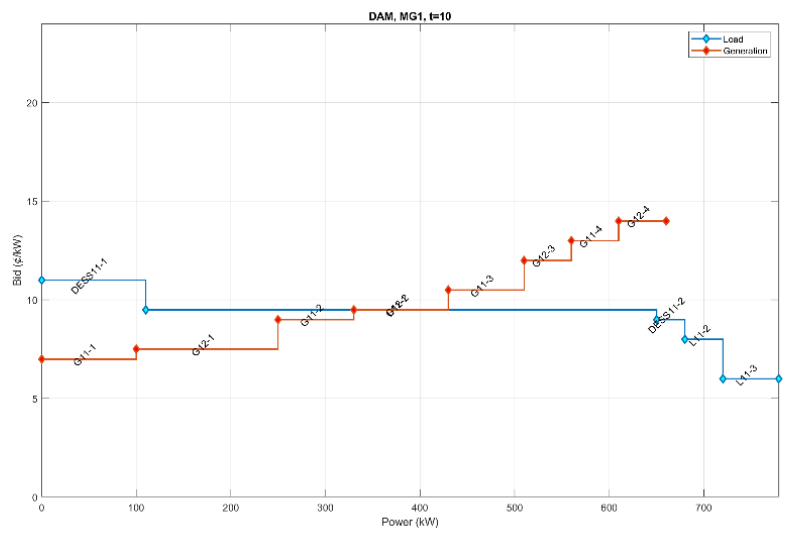

Round 1.

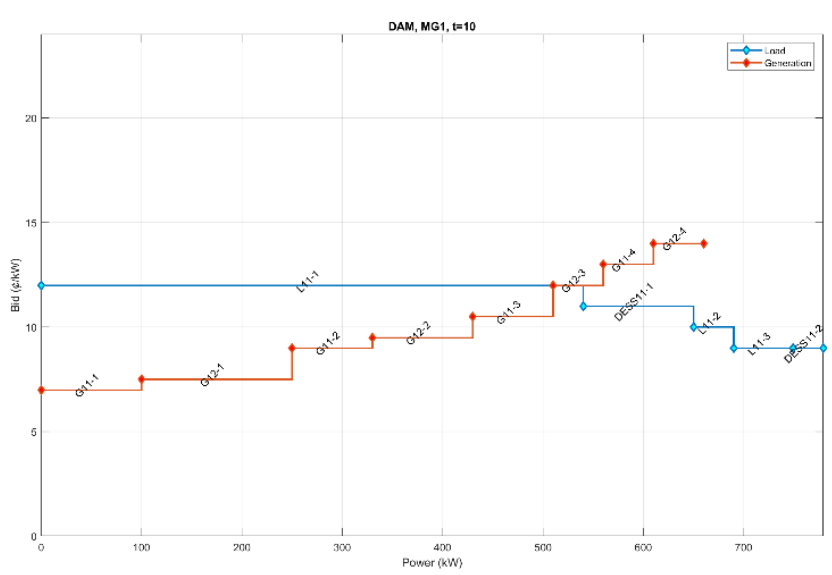

Round 2.

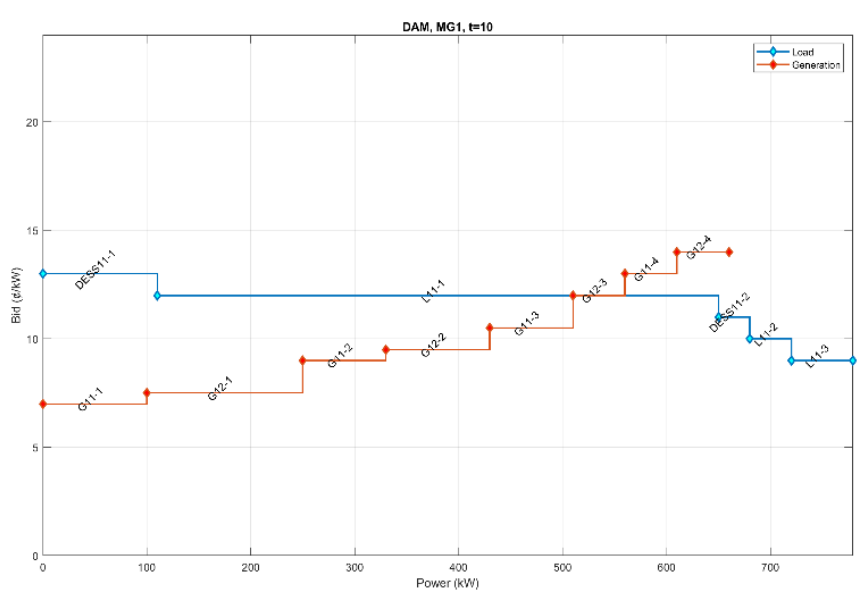

Round 3. 


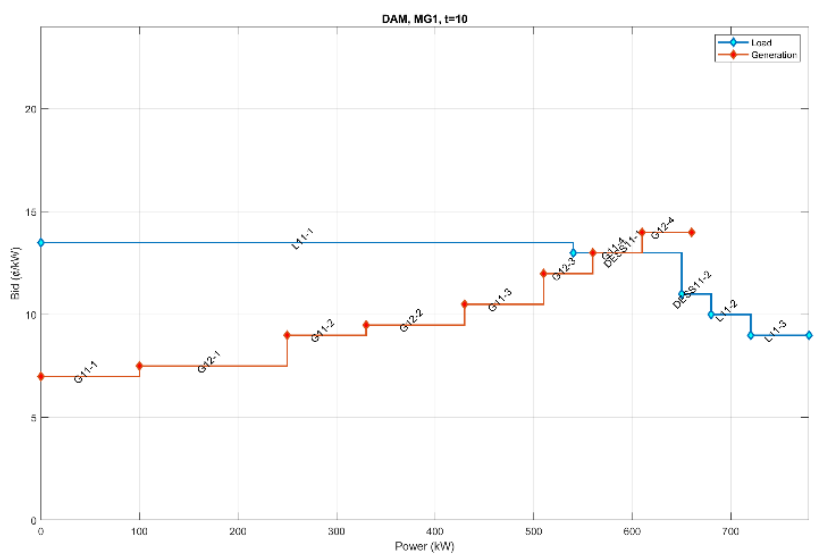

Round 4.

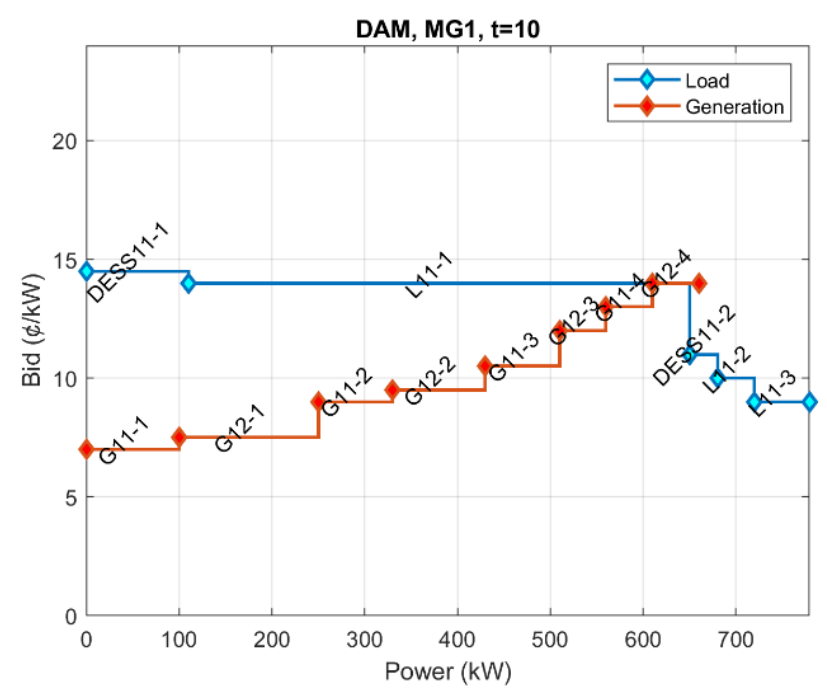

Round 5.

Figure 3: Double Auction Results 


\section{Appendix II: Developing the MgChain for Inter-MG transaction}

The developed HBC using Java programming language is called MgChain, which is developed for secure inter-MG transactions. Following we are presenting the developed codes step by step. It starts with creating transaction blocks followed by the cryptography algorithm, and wallet for each MG in the system. The transaction class and main MgChain codes are also provided in this section. The first step is to develop a block, where we can add transaction to the systems:

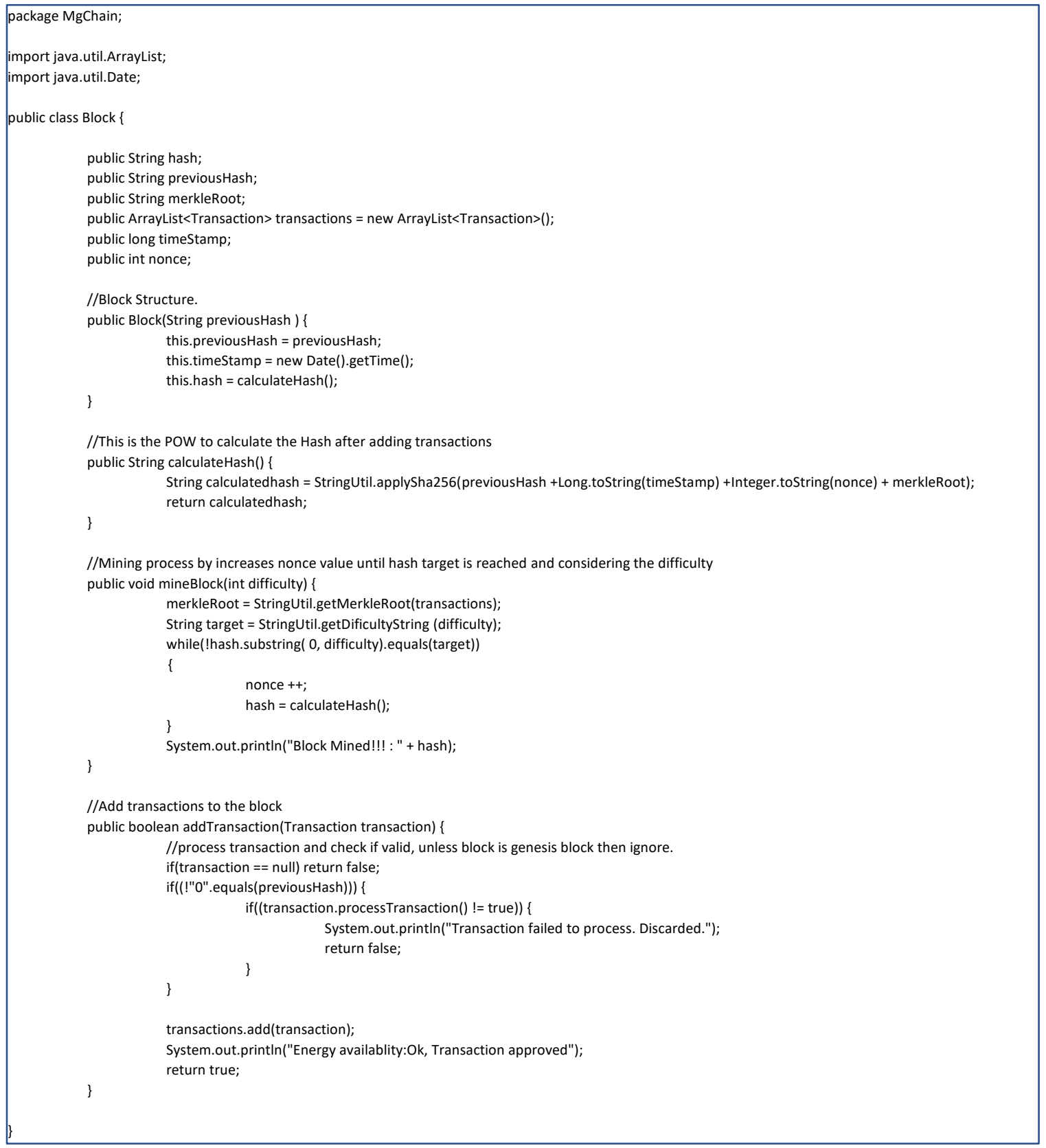


We used the SHA256 as our cryptographic algorithms to generate digital signature and Hash transaction blocks;

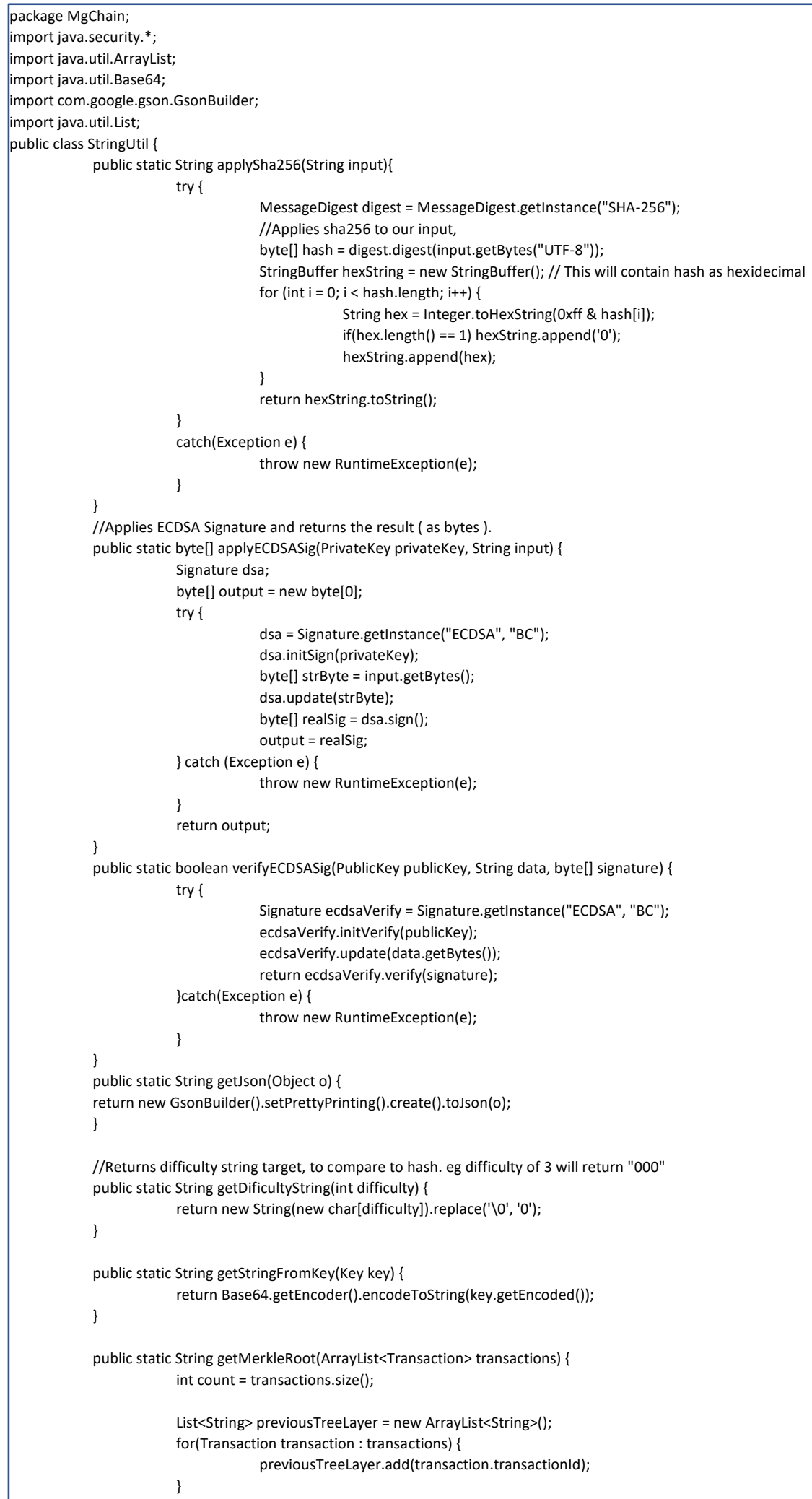


The Wallet, counting the available energy for each MG in the Inter-MG layer. The energy availability and transaction feasibility are checked here before approving them.

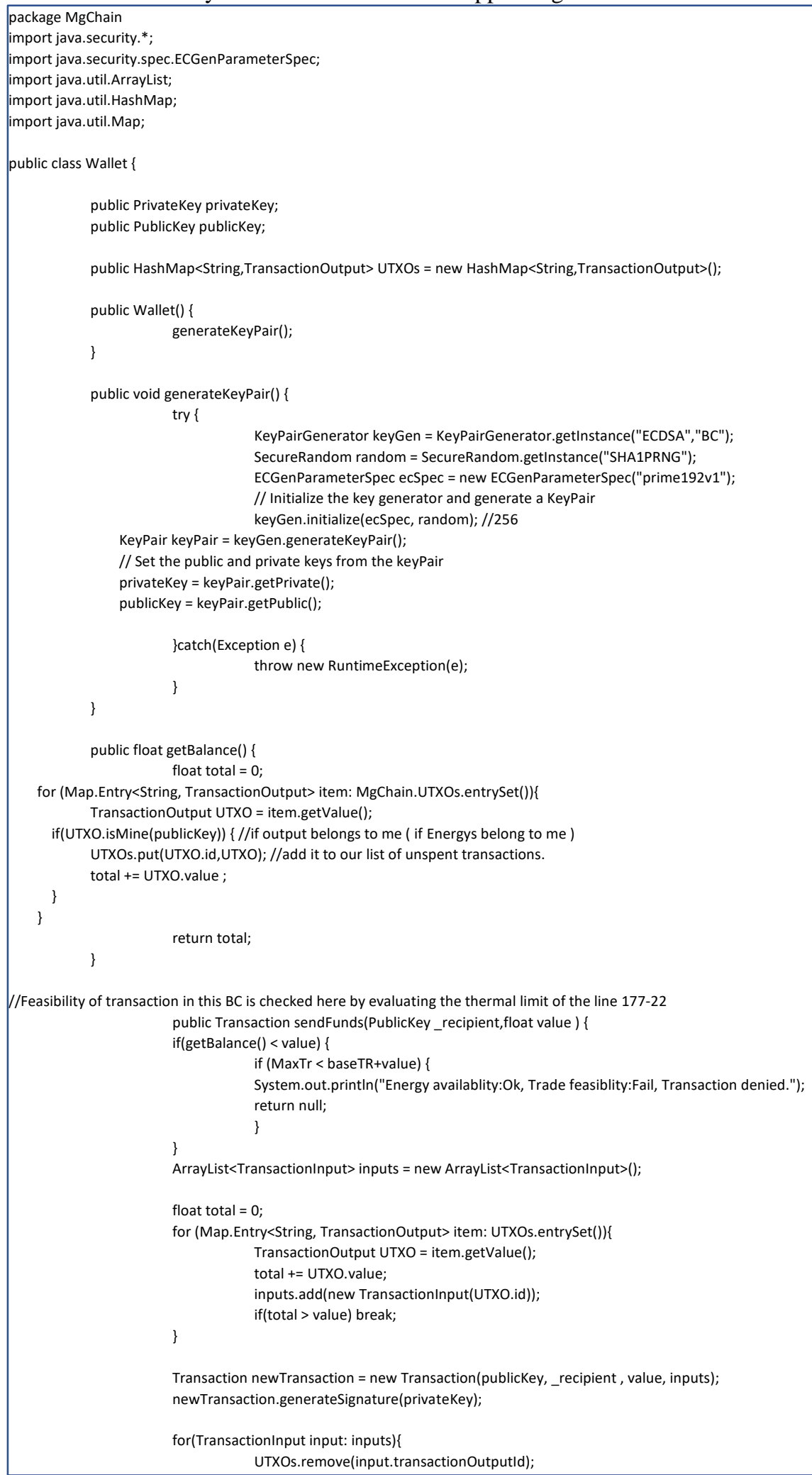


We have defined a class for transactions to build and make transactions among different wallets.

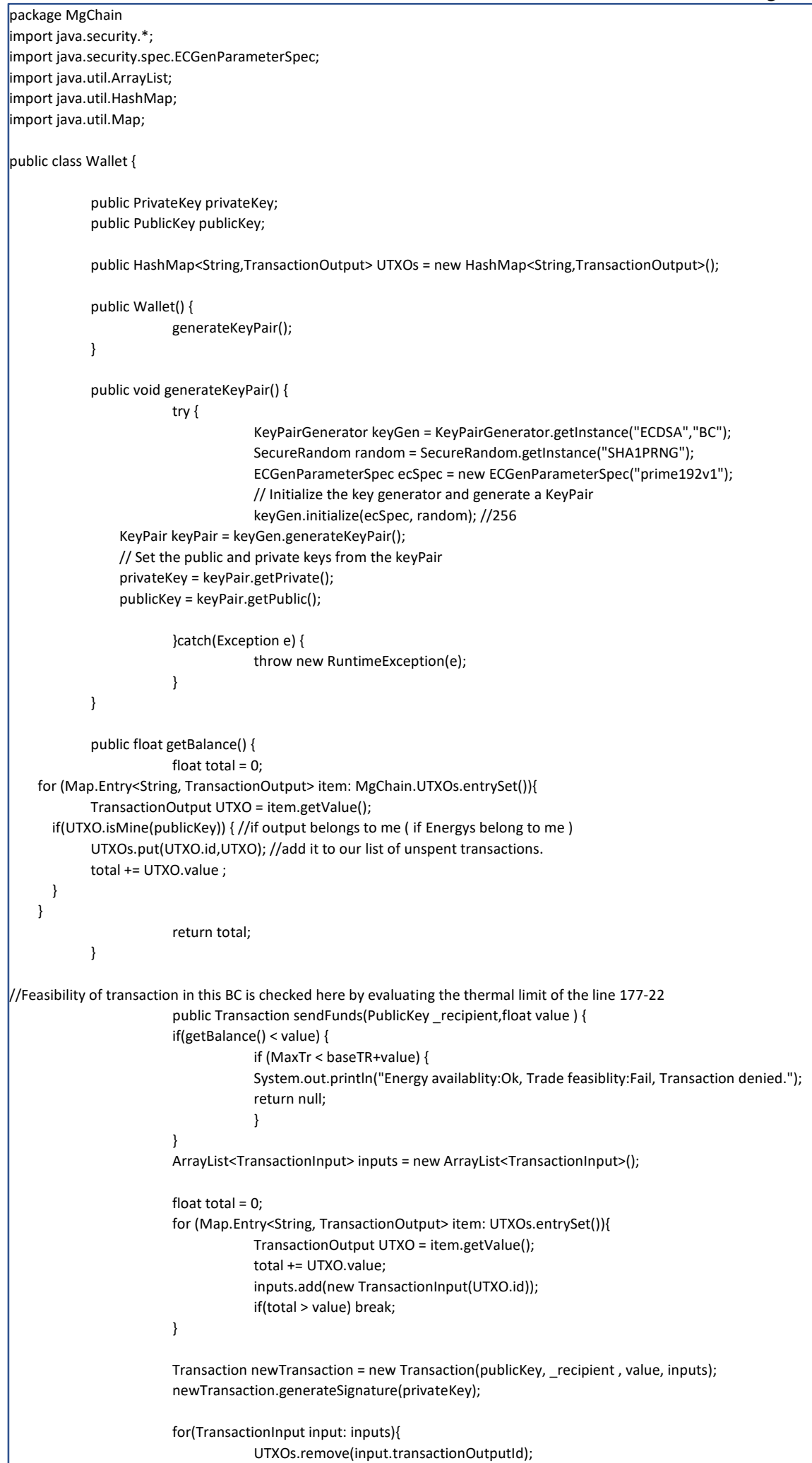


And finally, the MgChain, which implements all above mentioned class and objects to make transactions and add them to new blocks.

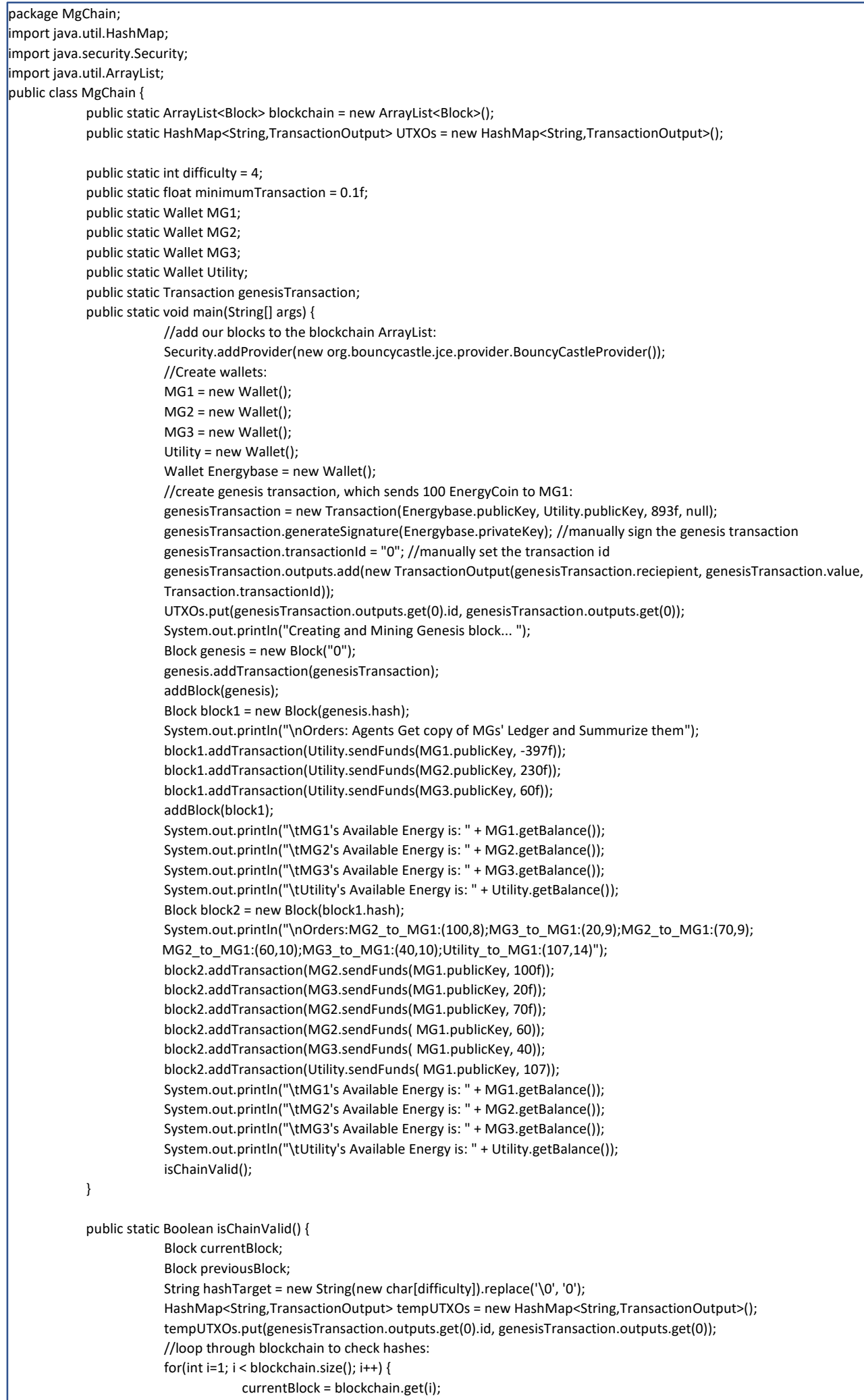




\section{Appendix III: Unbalanced Hybrid MGs}

System data for the modified IEEE 13-bus system: All data shown in Chapter 7 is shown by Table 1 to 4.

Table 1: Loads data for 8 PM

Terminal Power Factor Phase_A (kW) Phase_B (kW) Phase_C (kW)

\begin{tabular}{|c|c|c|c|c|}
\hline $\mathbf{6 8 0}$ & 0.97 & 33.3 & 271.7 & 39.3 \\
\hline $\mathbf{6 3 4 - L V 2}$ & 0.95 & 46.9 & 26.1 & 13.2 \\
\hline $\mathbf{6 3 4}$ & 0.98 & 59.4 & 112.2 & 112.2 \\
\hline $\mathbf{6 1 1}$ & 0.95 & 0.0 & 0.0 & 64.8 \\
\hline $\mathbf{6 4 6}$ & 0.40 & 0.0 & 76.2 & 0.0 \\
\hline $\mathbf{6 4 5 - L V - 1}$ & 0.44 & 6.6 & 0.0 & 0.0 \\
\hline DL1 & 0.87 & 1.9 & 7.4 & 13.1 \\
\hline DL2 & 0.87 & 0.2 & 0.7 & 1.3 \\
\hline DL3 & 0.87 & 2.4 & 9.2 & 16.3 \\
\hline DL4 & 0.87 & 1.7 & 6.6 & 11.7 \\
\hline DL5 & 0.87 & 0.6 & 2.4 & 4.2 \\
\hline $\mathbf{6 7 1}$ & 0.87 & 215.4 & 215.4 & 215.4 \\
\hline $\mathbf{6 1 1}$ & 0.90 & 0.0 & 0.0 & 91.2 \\
\hline $\mathbf{6 3 4 - L V 2}$ & 0.81 & 40.5 & 29.4 & 30.0 \\
\hline $\mathbf{6 3 4}$ & 0.81 & 79.7 & 59.8 & 59.8 \\
\hline $\mathbf{6 4 5 - L V - 1}$ & 0.81 & 42.4 & 0.0 & 0.0 \\
\hline $\mathbf{6 4 5 - L V - 2}$ & 0.81 & 0.0 & 46.1 & 0.0 \\
\hline $\mathbf{6 4 6}$ & 0.87 & 0.0 & 75.8 & 36.9 \\
\hline $\mathbf{6 5 2}$ & 0.83 & 66.4 & 0.0 & 0.0 \\
\hline $\mathbf{6 7 5}$ & 0.88 & 241.7 & 33.9 & 144.5 \\
\hline $\mathbf{6 9 2}$ & 0.75 & 0.0 & 0.0 & 63.1 \\
\hline $\mathbf{6 8 0}$ & 0.97 & 33.3 & 271.7 & 39.3 \\
\hline & & & & \\
\hline
\end{tabular}

Table 2: Transformers data

$\begin{array}{lllll}\text { Terminal i } & \text { Terminal j } & \text { HV (KV) } & \text { LV (KV) } & \text { Short-Circuit Voltage (\%) }\end{array}$

\begin{tabular}{|c|c|c|c|c|}
\hline Substation HV & 650 & 115 & 4.16 & 8.06 \\
\hline $\mathbf{6 4 5}$ & $645-\mathrm{LV}-1$ & 2.4 & 0.24 & 3 \\
\hline $\mathbf{6 3 3 - G e n}$ & 633 & 6.6 & 4.16 & 6 \\
\hline $\mathbf{6 3 3}$ & 634 & 4.16 & 0.48 & 2.28 \\
\hline
\end{tabular}

Table 3: Generation data for $8 \mathrm{PM}$

Terminal Controller Activ Pow. (KW) React.Pow. (Kvar) Voltage (p.u)

\begin{tabular}{|l|c|c|c|c|c}
\hline Ext. Grid & 650 & Swing & 1789.94 & 1170.09 & 1.00 \\
\hline DG & 633-Gen & P-V & 839.25 & 406.47 & 1.04 \\
\hline PV 634-1 & 634-LV1 & P-Q & 75.77 & 0.00 & - \\
\hline PV 634-2 & $634-L V 2$ & P-Q & 49.32 & 0.00 & - \\
\hline PV 645-1 & $645-L V 1$ & P-Q & 13.96 & 0.00 & - \\
\hline PV 645-2 & $645-L V 2$ & P-Q & 25.98 & 0.00 & - \\
\hline
\end{tabular}

Table 4: Lines data

\begin{tabular}{|c|c|c|c|c|c|}
\hline From & to & Length $(\mathbf{f t})$ & Rate $(\mathbf{K A})$ & R1 $(\mathbf{O h m})$ & X1 (Ohm) \\
\hline $\mathbf{6 3 4}$ & $634-\mathrm{LV} 1$ & 200 & 0.68 & 0.0050 & 0.0021 \\
\hline $\mathbf{6 3 4 - L V 1}$ & $634-\mathrm{LV} 2$ & 300 & 0.68 & 0.0075 & 0.0032 \\
\hline $\mathbf{6 8 4}$ & 652 & 800 & 0.165 & 0.2220 & 0.0795 \\
\hline $\mathbf{6 9 2}$ & 675 & 500 & 0.26 & 0.0340 & 0.0420 \\
\hline $\mathbf{6 3 2}$ & 633 & 500 & 0.68 & 0.0569 & 0.0719 \\
\hline $\mathbf{6 3 2}$ & 645 & 500 & 0.92 & 0.1068 & 0.0845 \\
\hline $\mathbf{6 3 2}$ & 671 & 2000 & 1.46 & 0.0117 & 0.0364 \\
\hline $\mathbf{6 4 5}$ & 646 & 300 & 0.92 & 0.0641 & 0.0507 \\
\hline $\mathbf{6 4 5 - L V - 1}$ & $645-\mathrm{LV}-2$ & 100 & 0.40 & 0.0030 & 0.0030 \\
\hline $\mathbf{R G 6 0}$ & 632 & 2000 & 1.46 & 0.0704 & 0.2187 \\
\hline $\mathbf{6 7 1}$ & 680 & 1000 & 1.46 & 0.0352 & 0.1093 \\
\hline $\mathbf{6 7 1}$ & 684 & 300 & 0.92 & 0.0641 & 0.0507 \\
\hline $\mathbf{6 8 4}$ & 611 & 300 & 0.92 & 0.0694 & 0.0921 \\
\hline $\mathbf{6 7 1}$ & 692 & 10 & 0.26 & 0.0007 & 0.0008 \\
\hline
\end{tabular}


Experimental Set up at FIU smart Grid Testbed.

The SCADA system art FIU smart grid testbed has bed developed by LabVIEW. The interface of this control center is shown by Figure 1. This file is located in the main PC at FIU smart grid testbed. This interface is supported by several DAQs for data collection and serial and wireless communication links to control all loads and generators in the testbed.

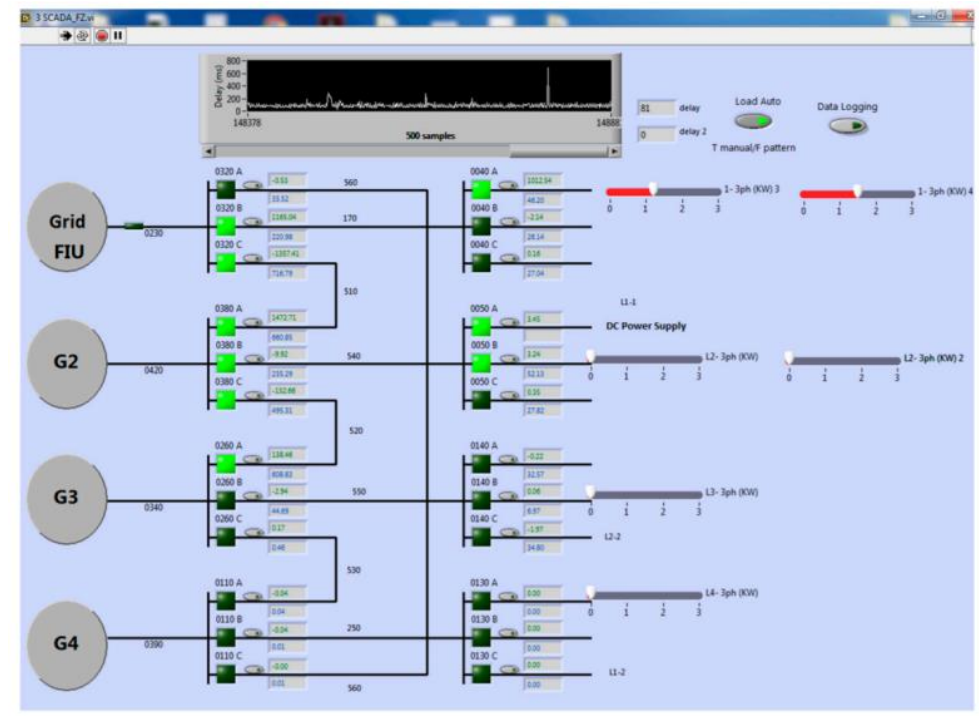

Figure 1: SCADA interface at FIU smart grid testbed

The optimization algorithm and system modeling were developed in DigSilent PowerFactory. So, following steps are needed to run the OPR in the experimental setup:

1. Generator's data and load values are recorded in the text files in a shared memory space.

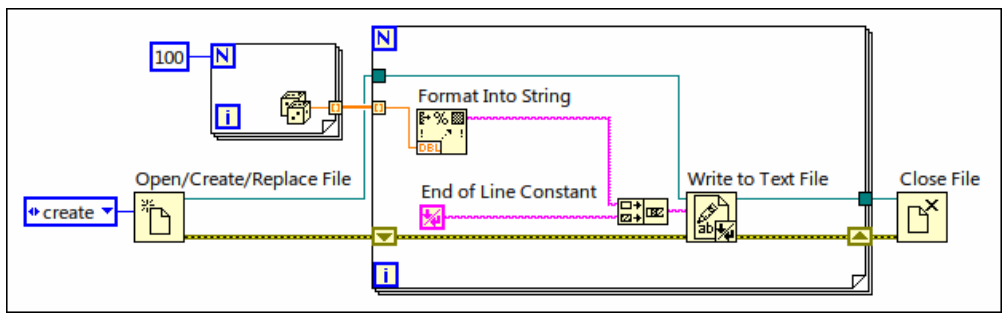

Figure 2: Write to the text file in LabView

2. The DigSilent PowerFactory reads this data and set the values for generates and load in the developed model. The file named ((Modified IEEE 13-Main work-IET- 
Dynamic)) contains the executive files. It worth mentioning that the name of the DPLs are based on the case studies of in Chapter 7.

3. It runs the unbalanced power flow to make sure the system model is working properly.

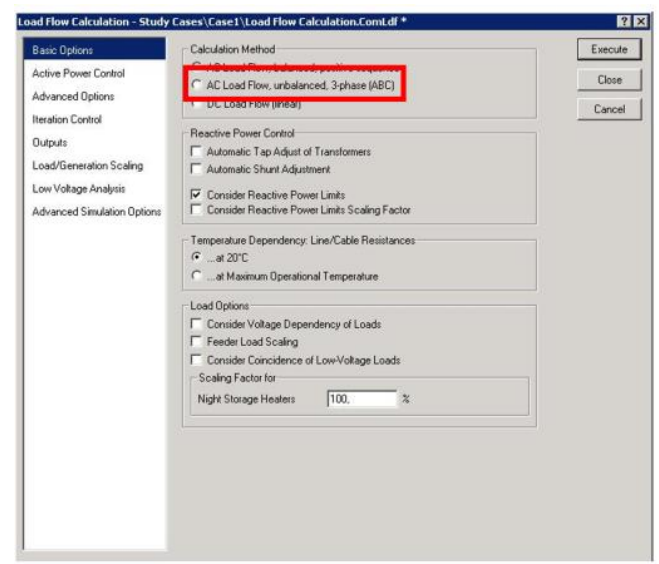

Figure 3: Unbalanced power flow calculation in DigSilent

4. Run the developed optimization DPL file to find the best load values for each CSs under the case study folder in DigSilent PowerFactory2019 installed on the main PC in the FIU Smart Grid Testbed.

5. The results will be exported to the text files by DPL.

6. The LabView reads the text file for each load through the following function which already has been modeled in the Testbed file LabView file called "MehranFile" .

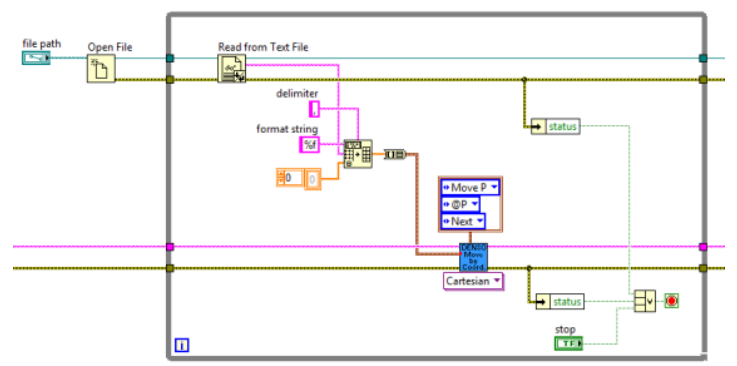

Figure 4: Read from a text file in LabView 
7. All measurement points in the developed LabView File are recorded data

\section{continuously in pre-defined Excel sheets.}

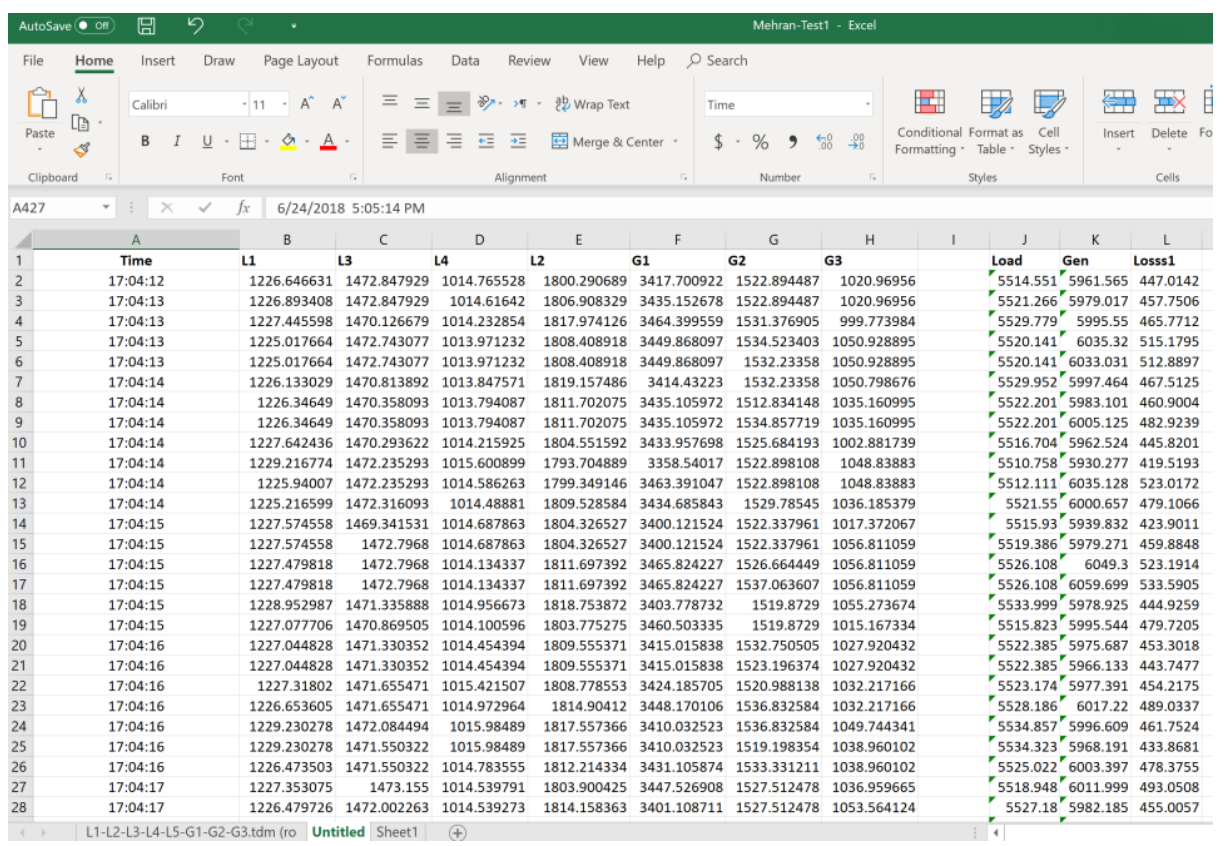

Figure 5: Raw data obtained from LabView for case 1.

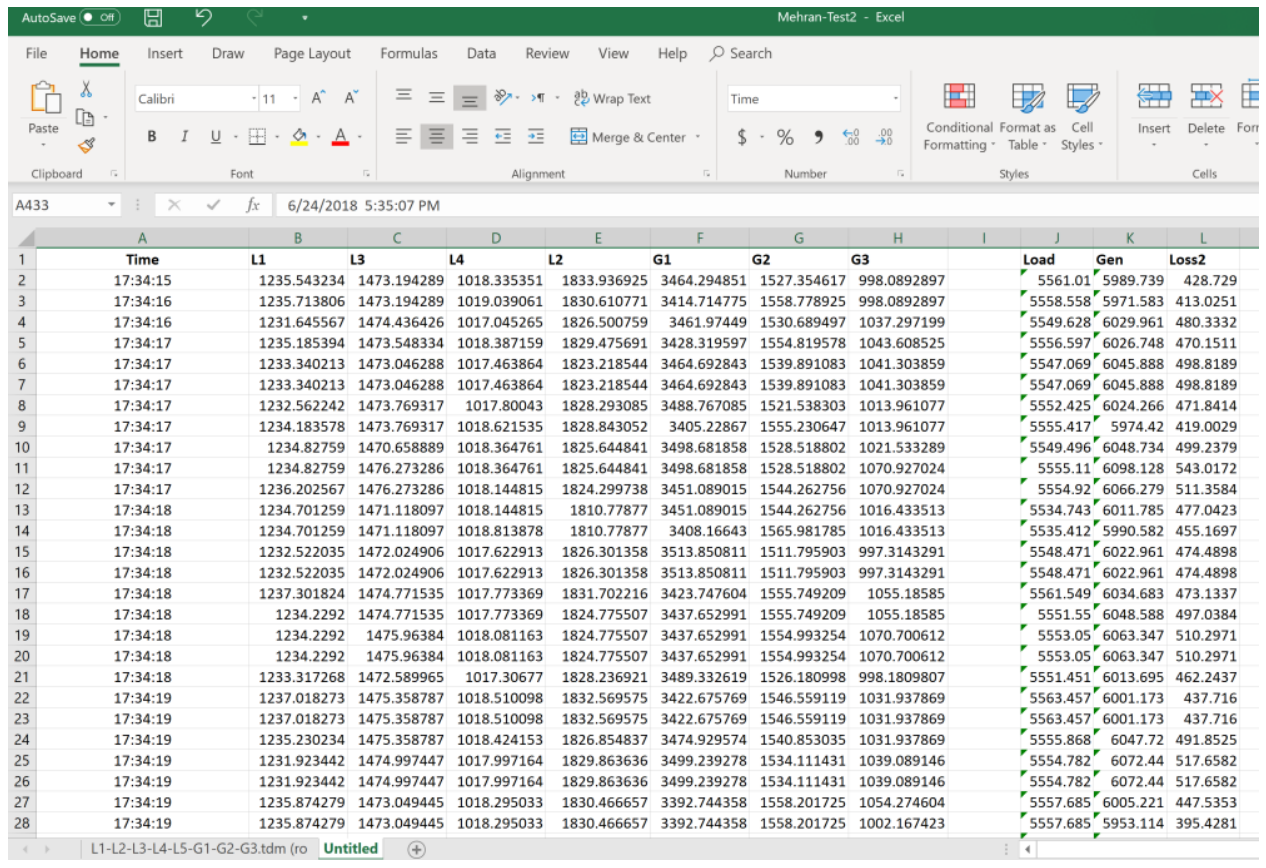

Figure 5: Raw data obtained from LabView for case 2. 
8. By analyzing, filtering bad data and comparing the results, practical results are obtained.

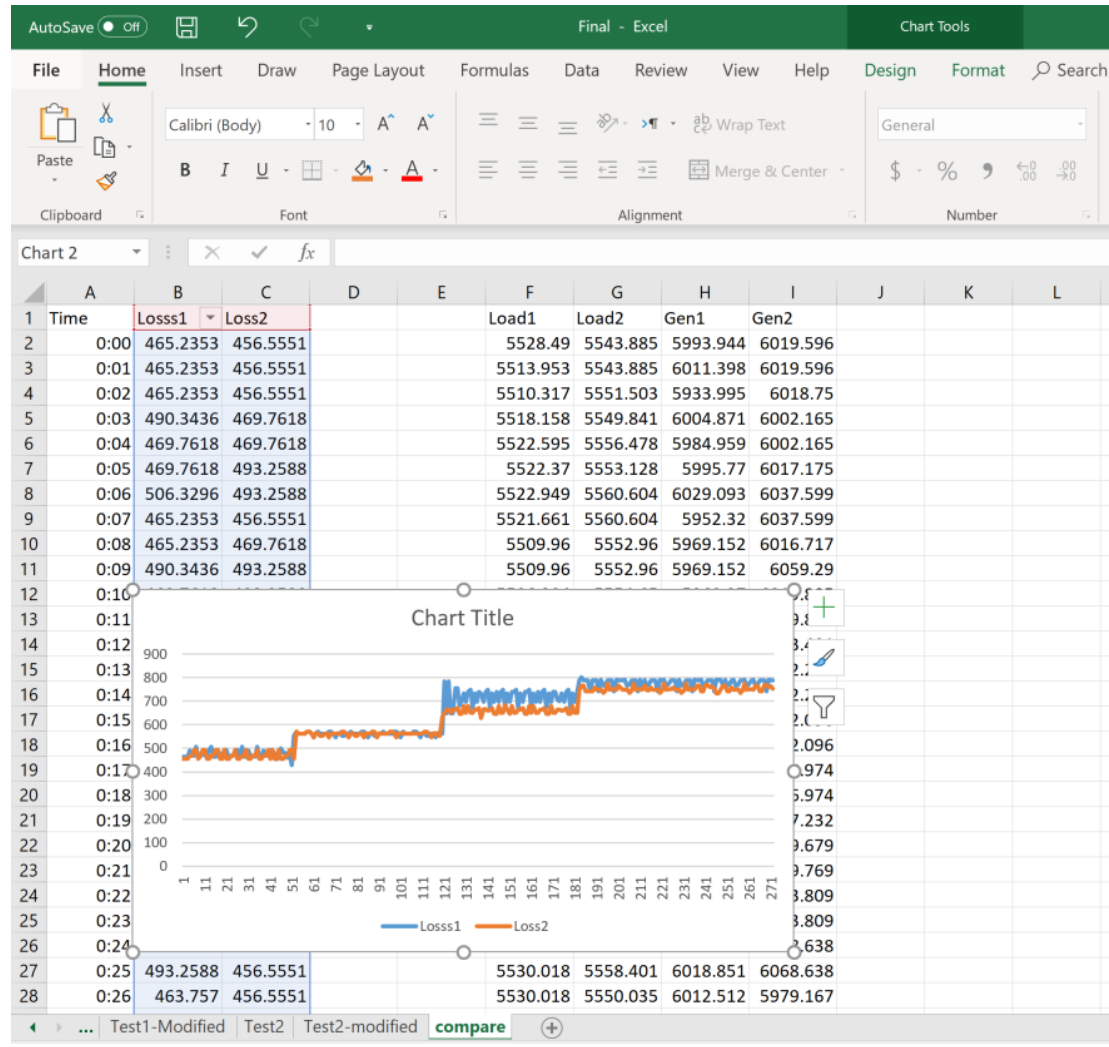

Figure 6: Data analyzing and comparison. 


\section{References}

[1] D. Gan, D. Feng and J. Xie, Electricity Markets and Power System Economics. CRC Press, 2013.

[2] K. Ranttila, "Social Media and Monopoly," Ohio NUL Rev., vol. 46, pp. 161, 2020.

[3] X. Zhang, Restructured Electric Power Systems: Analysis of Electricity Markets with Equilibrium Models. John Wiley \& Sons, 201071.

[4] J. Glachant and D. Finon, Competition in European Electricity Markets. Edward Elgar Publishing, 2003.

[5] Y. Wang and L. Li, "Time-of-use based electricity demand response for sustainable manufacturing systems," Energy, vol. 63, pp. 233-244, 2013.

[6] J. C. Jansen, K. Gialoglou and C. Egenhofer, Market Stimulation of Renewable Electricity in the EU: What Degree of Harmonisation of Support Mechanisms is Required? CEPS, 2005(56).

[7] M. H. Albadi and E. El-Saadany, "A summary of demand response in electricity markets," Electr. Power Syst. Res., vol. 78, (11), pp. 1989-1996, 2008.

[8] V. Sarkar and S. A. Khaparde, "A comprehensive assessment of the evolution of financial transmission rights," IEEE Trans. Power Syst., vol. 23, (4), pp. 1783-1795, 2008.

[9] R. Green, "Nodal pricing of electricity: how much does it cost to get it wrong?" Journal of Regulatory Economics, vol. 31, (2), pp. 125-149, 2007.

[10] S. Al Kaabi S., H. H. Zeineldin and V. Khadkikar, "Planning active distribution networks considering multi-DG configurations," IEEE Trans. Power Syst., vol. 29, (2), pp. 785-793, 2013.

[11] G. N. Taranto, T. M. L. Assis, D. M. Falcao and R. de Carvalho C., "Highlighting the importance of chronology on voltage protection and control in active distribution networks," IEEE Trans. Power Del., vol. 32, (1), pp. 361-369, 2016.

[12] I. Series, "Microgrids and active distribution networks," The Institution of Engineering and Technology, 2009.

[13] T. Lv and Q. Ai, "Interactive energy management of networked microgrids-based active distribution system considering large-scale integration of renewable energy resources," Appl. Energy, vol. 163, pp. 408-422, 2016. 
[14] I. Dincer, "Environmental impacts of energy," Energy Policy, vol. 27, (14), pp. 845854, 1999.

[15] C. L. Prete and B. F. Hobbs, "A cooperative game theoretic analysis of incentives for microgrids in regulated electricity markets," Appl. Energy, vol. 169, pp. 524-541, 2016.

[16] C. Phurailatpam, B. S. Rajpurohit and N. M. Pindoriya, "Embracing microgrids: Applications for rural and urban india," in 2015, .

[17] P. Mahat, Z. Chen and B. Bak-Jensen, "Review of islanding detection methods for distributed generation," in 2008, pp. 2743-2748.

[18] K. Alanne and A. Saari, "Distributed energy generation and sustainable development," Renewable and Sustainable Energy Reviews, vol. 10, (6), pp. 539-558, 2006.

[19] J. Jimeno, J. Anduaga, J. Oyarzabal and A. G. de Muro, "Architecture of a microgrid energy management system," European Transactions on Electrical Power, vol. 21, (2), pp. 1142-1158, 2011.

[20] E. Kuznetsova, Y. Li, C. Ruiz and E. Zio, "An integrated framework of agent-based modelling and robust optimization for microgrid energy management," Appl. Energy, vol. 129, pp. 70-88, 2014.

[21] Y. Parag and B. K. Sovacool, "Electricity market design for the prosumer era," Nature Energy, vol. 1, (4), pp. 1-6, 2016.

[22] V. Bertsch, M. Hall, C. Weinhardt and W. Fichtner, "Public acceptance and preferences related to renewable energy and grid expansion policy: Empirical insights for Germany," Energy, vol. 114, pp. 465-477, 2016.

[23] D. Ilic, P. G. Da Silva, S. Karnouskos and M. Griesemer, "An energy market for trading electricity in smart grid neighbourhoods," in 2012, pp. 1-6.

[24] S. Lamparter, S. Becher and J. Fischer, "An agent-based market platform for smart grids," in 2010, pp. 1689-1696.

[25] M. R. Blouin and R. Serrano, "A decentralized market with common values uncertainty: Non-steady states," The Review of Economic Studies, vol. 68, (2), pp. 323346, 2001.

[26] C. Block, D. Neumann and C. Weinhardt, "A market mechanism for energy allocation in micro-chp grids," in 2008, pp. 172. 
[27] P. Vytelingum, S. D. Ramchurn, T. D. Voice, A. Rogers and N. R. Jennings, "Trading agents for the smart electricity grid," 2010.

[28] H. Algarvio, F. Lopes, J. Sousa and J. Lagarto, "Multi-agent electricity markets: Retailer portfolio optimization using Markowitz theory," Electr. Power Syst. Res., vol. 148, pp. 282-294, 2017.

[29] H. K. Nunna and S. Doolla, "Energy management in microgrids using demand response and distributed storage-A multiagent approach," IEEE Trans. Power Del., vol. 28, (2), pp. 939-947, 2013.

[30] E. Karfopoulos, L. Tena, A. Torres, P. Salas, J. G. Jorda, A. Dimeas and N. Hatziargyriou, "A multi-agent system providing demand response services from residential consumers," Electr. Power Syst. Res., vol. 120, pp. 163-176, 2015.

[31] Nunna, Kumar H. S. V. S. and S. Doolla, "Responsive end-user-based demand side management in multimicrogrid environment," IEEE Transactions on Industrial Informatics, vol. 10, (2), pp. 1262-1272, 2014.

[32] D. Wang, X. Guan, J. Wu, P. Li, P. Zan and H. Xu, "Integrated energy exchange scheduling for multimicrogrid system with electric vehicles," IEEE Transactions on Smart Grid, vol. 7, (4), pp. 1762-1774, 2015.

[33] Y. Wang, S. Mao and R. M. Nelms, "On hierarchical power scheduling for the macrogrid and cooperative microgrids," IEEE Transactions on Industrial Informatics, vol. 11, (6), pp. 1574-1584, 2015.

[34] J. Lee, J. Guo, J. K. Choi and M. Zukerman, "Distributed energy trading in microgrids: A game-theoretic model and its equilibrium analysis," IEEE Trans. Ind. Electron., vol. 62, (6), pp. 3524-3533, 2015.

[35] D. Gregoratti and J. Matamoros, "Distributed energy trading: The multiplemicrogrid case," IEEE Trans. Ind. Electron., vol. 62, (4), pp. 2551-2559, 2014.

[36] C. Dou, D. Yue, X. Li and Y. Xue, "MAS-based management and control strategies for integrated hybrid energy system," IEEE Transactions on Industrial Informatics, vol. 12, (4), pp. 1332-1349, 2016.

[37] J. Shen, C. Jiang, Y. Liu and X. Wang, "A microgrid energy management system and risk management under an electricity market environment," IEEE Access, vol. 4, pp. 2349-2356, 2016.

[38] S. Park, J. Lee, S. Bae, G. Hwang and J. K. Choi, "Contribution-based energytrading mechanism in microgrids for future smart grid: A game theoretic approach," IEEE Trans. Ind. Electron., vol. 63, (7), pp. 4255-4265, 2016. 
[39] S. Park, J. Lee, G. Hwang and J. K. Choi, "Event-driven energy trading system in microgrids: Aperiodic market model analysis with a game theoretic approach," IEEE Access, vol. 5, pp. 26291-26302, 2017.

[40] A. Sesetti, H. Nunna, S. Doolla and A. K. Rathore, "Designing energy auction market for inclusion of storage systems and quantifying their impact on network lossesMulti agent approach," in 2017, pp. 1-8.

[41] H. K. Nunna and D. Srinivasan, "Multiagent-based transactive energy framework for distribution systems with smart microgrids," IEEE Transactions on Industrial Informatics, vol. 13, (5), pp. 2241-2250, 2017.

[42] V. Bui, A. Hussain and H. Kim, "A multiagent-based hierarchical energy management strategy for multi-microgrids considering adjustable power and demand response," IEEE Transactions on Smart Grid, vol. 9, (2), pp. 1323-1333, 2016.

[43] M. H. Cintuglu, H. Martin and O. A. Mohammed, "Real-time implementation of multiagent-based game theory reverse auction model for microgrid market operation," IEEE Transactions on Smart Grid, vol. 6, (2), pp. 1064-1072, 2015.

[44] S. Nakamoto, "Bitcoin: A peer-to-peer electronic cash system," Manubot, 2019.

[45] F. Hasse, A. Von Perfall, T. Hillebrand, E. Smole, L. Lay and M. Charlet, "Blockchain-an opportunity for energy producers and consumers," PwC Global Power \& Utilities, pp. 1-45, 2016.

[46] M. Crosby, P. Pattanayak, S. Verma and V. Kalyanaraman, "Blockchain technology: Beyond bitcoin," Applied Innovation, vol. 2, (6-10), pp. 71, 2016.

[47] G. Greenspan, "Ending the bitcoin vs blockchain debate," Retrieved November, vol. 1, pp. 2018, 2015.

[48] F. Tschorsch and B. Scheuermann, "Bitcoin and beyond: A technical survey on decentralized digital currencies," IEEE Communications Surveys \& Tutorials, vol. 18, (3), pp. 2084-2123, 2016.

[49] Y. Xu, S. Zhao, L. Kong, Y. Zheng, S. Zhang and Q. Li, "ECBC: A high performance educational certificate blockchain with efficient query," in 2017, pp. 288304.

[50] M. Crosby, P. Pattanayak, S. Verma and V. Kalyanaraman, "Blockchain technology: Beyond bitcoin," Applied Innovation, vol. 2, (6-10), pp. 71, 2016.

[51] M. Swan, Blockchain: Blueprint for a New Economy. " O'Reilly Media, Inc.", 2015. 
[52] M. Pilkington, "Blockchain technology: Principles and applications," in Research Handbook on Digital Transformations Anonymous Edward Elgar Publishing, 2016, .

[53] S. Wang, L. Ouyang, Y. Yuan, X. Ni, X. Han and F. Wang, "Blockchain-enabled smart contracts: architecture, applications, and future trends," IEEE Transactions on Systems, Man, and Cybernetics: Systems, vol. 49, (11), pp. 2266-2277, 2019.

[54] J. J. Sikorski, J. Haughton and M. Kraft, "Blockchain technology in the chemical industry: Machine-to-machine electricity market," Appl. Energy, vol. 195, pp. 234-246, 2017.

[55] N. Szabo, "Smart contracts: building blocks for digital markets," EXTROPY: The Journal of Transhumanist Thought,(16), vol. 18, pp. 2, 1996.

[56] K. Christidis and M. Devetsikiotis, "Blockchains and smart contracts for the internet of things," Ieee Access, vol. 4, pp. 2292-2303, 2016.

[57] M. Conoscenti, A. Vetro and J. C. De Martin, "Blockchain for the internet of things: A systematic literature review," in 2016, pp. 1-6.

[58] E. Karafiloski and A. Mishev, "Blockchain solutions for big data challenges: A literature review," in 2017, pp. 763-768.

[59] M. A. Khan and K. Salah, "IoT security: Review, blockchain solutions, and open challenges," Future Generation Comput. Syst., vol. 82, pp. 395-411, 2018.

[60] S. Seebacher and R. Schüritz, "Blockchain technology as an enabler of service systems: A structured literature review," in 2017, pp. 12-23.

[61] N. Atzei, M. Bartoletti and T. Cimoli, "A survey of attacks on ethereum smart contracts (sok)," in 2017, pp. 164-186.

[62] F. Casino, T. K. Dasaklis and C. Patsakis, "A systematic literature review of blockchain-based applications: current status, classification and open issues," Telematics Inf., vol. 36, pp. 55-81, 2019.

[63] Q. K. Nguyen, "Blockchain-a financial technology for future sustainable development," in 2016, pp. 51-54.

[64] W. Reijers, F. O'Brolcháin and P. Haynes, "Governance in blockchain technologies \& social contract theories," Ledger, vol. 1, pp. 134-151, 2016.

[65] R. McMillan, "Hacker Dreams Up Crypto Passport Using the Tech Behind Bitcoin," 2014. 
[66] R. Rivera, J. G. Robledo, V. Larios and J. M. Avalos, "How digital identity on blockchain can contribute in a smart city environment," in 2017, pp. 1-4.

[67] J. Killmeyer, M. White and B. Chew, "Will blockchain transform the public sector? Blockchain basics for government," Deloitte Center for Government Insights, 2017.

[68] P. Boucher, "What if blockchain technology revolutionised voting," Unpublished Manuscript, European Parliament, 2016.

[69] T. Moura and A. Gomes, "Blockchain voting and its effects on election transparency and voter confidence," in 2017, pp. 574-575.

[70] J. Deitz, "Decentralized Governance Whitepaper," Quora, May, vol. 21, 2014.

[71] P. Noizat, "Blockchain electronic vote," in Handbook of Digital CurrencyAnonymous Elsevier, 2015, pp. 453-461.

[72] M. Mettler, "Blockchain technology in healthcare: The revolution starts here," in 2016, pp. 1-3.

[73] P. Urien, "Blockchain IoT (BIoT): A new direction for solving internet of things security and trust issues," in 2018, pp. 1-4.

[74] N. Kshetri, "Blockchain's roles in strengthening cybersecurity and protecting privacy," Telecommun. Policy, vol. 41, (10), pp. 1027-1038, 2017.

[75] D. Tapscott and A. Tapscott, "How blockchain will change organizations," MIT Sloan Management Review, vol. 58, (2), pp. 10, 2017.

[76] R. Pass and A. Shelat, "Micropayments for decentralized currencies," in 2015, pp. 207-218.

[77] T. Ruffing, P. Moreno-Sanchez and A. Kate, "Coinshuffle: Practical decentralized coin mixing for bitcoin," in 2014, pp. 345-364.

[78] B. Liu, X. L. Yu, S. Chen, X. Xu and L. Zhu, "Blockchain based data integrity service framework for IoT data," in 2017, pp. 468-475.

[79] E. Parliament and E. Council, "General data protection regulation," in 2016, .

[80] M. Haferkorn and J. M. Q. Diaz, "Seasonality and interconnectivity within cryptocurrencies-an analysis on the basis of bitcoin, litecoin and namecoin," in 2014, pp. 106-120. 
[81] S. Asharaf and S. Adarsh, Decentralized Computing using Blockchain Technologies and Smart Contracts: Emerging Research and Opportunities: Emerging Research and Opportunities. IGI Global, 2017.

[82] G. Fridgen, S. Radszuwill, N. Urbach and L. Utz, "Cross-organizational workflow management using blockchain technology-towards applicability, auditability, and automation," 2018.

[83] M. Sharples and J. Domingue, "The blockchain and kudos: A distributed system for educational record, reputation and reward," in 2016, pp. 490-496.

[84] T. Ahram, A. Sargolzaei, S. Sargolzaei, J. Daniels and B. Amaba, "Blockchain technology innovations," in 2017, pp. 137-141.

[85] N. Hackius and M. Petersen, "Blockchain in logistics and supply chain: Trick or treat?" in 2017, pp. 3-18.

[86] K. Bilal, S. U. R. Malik, O. Khalid, A. Hameed, E. Alvarez, V. Wijaysekara, R. Irfan, S. Shrestha, D. Dwivedy and M. Ali, "A taxonomy and survey on green data center networks," Future Generation Comput. Syst., vol. 36, pp. 189-208, 2014.

[87] C. Burger, A. Kuhlmann, P. Richard and J. Weinmann, "Blockchain in the energy transition. A survey among decision-makers in the German energy industry," DENA German Energy Agency, vol. 60, 2016.

[88] E. Mengelkamp, B. Notheisen, C. Beer, D. Dauer and C. Weinhardt, "A blockchainbased smart grid: towards sustainable local energy markets," Computer Science-Research and Development, vol. 33, (1-2), pp. 207-214, 2018.

[89] J. A. Castellanos, D. Coll-Mayor and J. A. Notholt, "Cryptocurrency as guarantees of origin: Simulating a green certificate market with the ethereum blockchain," in 2017, pp. 367-372.

[90] S. Cheng, B. Zeng and Y. Z. Huang, "Research on application model of blockchain technology in distributed electricity market," in 2017, pp. 012065.

[91] M. Mihaylov, S. Jurado, N. Avellana, K. Van Moffaert, I. M. de Abril and A. Nowé, "NRGcoin: Virtual currency for trading of renewable energy in smart grids," in 2014, pp. $1-6$.

[92] V. Mohan, J. G. Singh and W. Ongsakul, "An efficient two stage stochastic optimal energy and reserve management in a microgrid," Appl. Energy, vol. 160, pp. 28-38, 2015. 
[93] E. Al Kawasmi, E. Arnautovic and D. Svetinovic, "Bitcoin-based decentralized carbon emissions trading infrastructure model," Systems Engineering, vol. 18, (2), pp. 115-130, 2015.

[94] N. Z. Aitzhan and D. Svetinovic, "Security and privacy in decentralized energy trading through multi-signatures, blockchain and anonymous messaging streams," IEEE Transactions on Dependable and Secure Computing, vol. 15, (5), pp. 840-852, 2016.

[95] E. Mengelkamp, J. Gärttner, K. Rock, S. Kessler, L. Orsini and C. Weinhardt, "Designing microgrid energy markets: A case study: The Brooklyn Microgrid," Appl. Energy, vol. 210, pp. 870-880, 2018.

[96] R. Beck, J. Stenum Czepluch, N. Lollike and S. Malone, "Blockchain-the gateway to trust-free cryptographic transactions," 2016.

[97] F. Glaser and L. Bezzenberger, "Beyond cryptocurrencies-a taxonomy of decentralized consensus systems," in 2015, .

[98] M. Pilkington, "Blockchain technology: Principles and applications," in Research Handbook on Digital Transformations Anonymous Edward Elgar Publishing, 2016, .

[99] K. O'Dwyer J. and D. Malone, "Bitcoin mining and its energy footprint," 2014.

[100] J. Green and P. Newman, "Citizen utilities: The emerging power paradigm," Energy Policy, vol. 105, pp. 283-293, 2017.

[101] M. M. Esfahani and G. R. Yousefi, "Real time congestion management in power systems considering quasi-dynamic thermal rating and congestion clearing time," IEEE Transactions on Industrial Informatics, vol. 12, (2), pp. 745-754, 2016.

[102] M. M. Esfahani, M. H. Cintuglu and O. A. Mohammed, "Optimal real-time congestion management in power markets based on particle swarm optimization," in 2017, pp. 1-5.

[103] H. Glatvitsch and F. Alvarado, "Management of multiple congested conditions in unbundled operation of a power system," IEEE Trans. Power Syst., vol. 13, (3), pp. 10131019, 1998.

[104] S. Dutta and S. P. Singh, "Optimal rescheduling of generators for congestion management based on particle swarm optimization," IEEE Trans. Power Syst., vol. 23, (4), pp. 1560-1569, 2008.

[105] N. Eghtedarpour and E. Farjah, "Power control and management in a hybrid AC/DC microgrid," IEEE Transactions on Smart Grid, vol. 5, (3), pp. 1494-1505, 2014. 
[106] A. A. Hamad, M. A. Azzouz and E. El Saadany F., "A sequential power flow algorithm for islanded hybrid AC/DC microgrids," IEEE Trans. Power Syst., vol. 31, (5), pp. 3961-3970, 2015.

[107] E. Aprilia, K. Meng, M. Al Hosani, H. H. Zeineldin and Z. Y. Dong, "Unified power flow algorithm for standalone AC/DC hybrid microgrids," IEEE Transactions on Smart Grid, vol. 10, (1), pp. 639-649, 2017.

[108] X. Lu, J. M. Guerrero, K. Sun, J. C. Vasquez, R. Teodorescu and L. Huang, "Hierarchical control of parallel AC-DC converter interfaces for hybrid microgrids," IEEE Transactions on Smart Grid, vol. 5, (2), pp. 683-692, 2013.

[109] M. Baharizadeh, H. R. Karshenas and J. M. Guerrero, "Control strategy of interlinking converters as the key segment of hybrid AC-DC microgrids," IET Generation, Transmission \& Distribution, vol. 10, (7), pp. 1671-1681, 2016.

[110] M. A. Allam, A. A. Hamad, M. Kazerani and E. El-Saadany, "A novel dynamic power routing scheme to maximize loadability of islanded hybrid AC/DC microgrids under unbalanced AC loading," IEEE Transactions on Smart Grid, vol. 9, (6), pp. 57985809, 2017.

[111] Y. Han, P. Shen, X. Zhao and J. M. Guerrero, "An enhanced power sharing scheme for voltage unbalance and harmonics compensation in an islanded AC microgrid," IEEE Trans. Energy Convers., vol. 31, (3), pp. 1037-1050, 2016.

[112] M. Hamzeh, H. Karimi and H. Mokhtari, "A new control strategy for a multi-bus MV microgrid under unbalanced conditions," IEEE Trans. Power Syst., vol. 27, (4), pp. 2225-2232, 2012.

[113] P. C. Loh, D. Li, Y. K. Chai and F. Blaabjerg, "Autonomous operation of hybrid microgrid with AC and DC subgrids," IEEE Transactions on Power Electronics, vol. 28, (5), pp. 2214-2223, 2012.

[114] A. Micallef, M. Apap, C. Spiteri-Staines and J. M. Guerrero, "Single-phase microgrid with seamless transition capabilities between modes of operation," IEEE Transactions on Smart Grid, vol. 6, (6), pp. 2736-2745, 2015.

[115] M. Savaghebi, A. Jalilian, J. C. Vasquez and J. M. Guerrero, "Autonomous voltage unbalance compensation in an islanded droop-controlled microgrid," IEEE Trans. Ind. Electron., vol. 60, (4), pp. 1390-1402, 2012.

[116] M. M. Rezaei and J. Soltani, "A robust control strategy for a grid-connected multibus microgrid under unbalanced load conditions," International Journal of Electrical Power \& Energy Systems, vol. 71, pp. 68-76, 2015. 
[117] H. Shi, F. Zhuo, H. Yi and Z. Geng, "Control strategy for microgrid under threephase unbalance condition," Journal of Modern Power Systems and Clean Energy, vol. 4, (1), pp. 94-102, 2016.

[118] M. M. Esfahani and O. A. Mohammed, "Loadability improvement of unbalanced hybrid ac-dc microgrids using a supervisory control scheme for interlinking converters," in 2018, pp. 1129-1134.

[119] M. M. Esfahani, H. F. Habib and O. A. Mohammed, "A hierarchical power routing scheme for interlinking converters in unbalanced hybrid AC-DC microgrids," in 2018, pp. 53-58.

[120] J. D. McDonald, "Substation automation. IED integration and availability of information," IEEE Power and Energy Magazine, vol. 1, (2), pp. 22-31, 2003.

[121] J. Horalek, J. Matyska and V. Sobeslav, "Communication protocols in substation automation and IEC 61850 based proposal," in 2013, pp. 321-326.

[122] R. E. Mackiewicz, "Overview of IEC 61850 and benefits," in 2006, pp. 8-pp.

[123] H. ZHAO and J. ZHANG, "Application of IEC 60870-5-101 Telecontrol Protocol in Data Communication of Distribution Automation System [J]," Power System Technology, vol. 11, 2006.

[124] F. Halsall, Data Communications, Computer Networks and Open Systems. Addison Wesley Longman Publishing Co., Inc, 1995.

[125] A. Swales, "Open modbus/tcp specification," Schneider Electric, vol. 29, 1999.

[126] G. Clarke, D. Reynders and E. Wright, Practical Modern SCADA Protocols: DNP3, 60870.5 and Related Systems. Newnes, 2004.

[127] A. Apostolov, "R-GOOSE: what it is and its application in distribution automation," CIRED-Open Access Proceedings Journal, vol. 2017, (1), pp. 1438-1441, 2017.

[128] A. Alaerjan and D. Kim, "Tailoring dds to smart grids for improved communication and control," in 2016, pp. 1-6.

[129] X. Cheng, W. Lee and X. Pan, "Electrical substation automation system modernization through the adoption of IEC61850," in 2015, pp. 1-7.

[130] S. R. Firouzi, L. Vanfretti, A. Ruiz-Alvarez, H. Hooshyar and F. Mahmood, "Interpreting and implementing IEC 61850-90-5 Routed-Sampled Value and Routed- 
GOOSE protocols for IEEE C37. 118.2 compliant wide-area synchrophasor data transfer," Electr. Power Syst. Res., vol. 144, pp. 255-267, 2017.

[131] I. Calvo, O. G. de Albéniz and F. Pérez, "A communication backbone for Substation Automation Systems based on the OMG DDS standard," Przeglad Elektrotechniczny, vol. 88, (1), pp. 146-150, 2012.

[132] H. V. Mendis, P. E. Heegaard and K. Kralevska, "5G Network Slicing as an Enabler for Smart Distribution Grid Operations," 2019.

[133] D. Berbecaru, "On measuring SSL-based secure data transfer with handheld devices," in 2005, pp. 409-413.

[134] M. Kanabar, A. Cioraca and A. Johnson, "Wide area protection \& control using high-speed and secured routable GOOSE mechanism," in 2016, pp. 1-6.

[135] K. V. Babu, M. Tripathy and A. K. Singh, "Recent techniques used in transmission line protection: a review," International Journal of Engineering, Science and Technology, vol. 3, (3), 2011.

[136] Y. G. Paithankar, Transmission Network Protection: Theory and Practice. CRC Press, 19972.

[137] R. Velásquez Manuel Arias and J. V. M. Lara, "Circuit breakers 500 kV degradation in substation reactors caused by inductive current," Eng. Failure Anal., vol. 90, pp. 64-81, 2018.

[138] M. Hasanuzzaman, U. S. Zubir, N. I. Ilham and H. Seng Che, "Global electricity demand, generation, grid system, and renewable energy polices: a review," Wiley Interdisciplinary Reviews: Energy and Environment, vol. 6, (3), pp. e222, 2017.

[139] A. Esmaeili, S. Esmaeili and H. Hojabri, "Short-circuit level control through a multi-objective feeder reconfiguration using fault current limiters in the presence of distributed generations," IET Generation, Transmission \& Distribution, vol. 10, (14), pp. 3458-3469, 2016.

[140] H. Falk, IEC 61850 Demystified. Artech House, 2018.

[141] T. S. Ustun, C. Ozansoy and A. Zayegh, "Extending IEC 61850-7-420 for distributed generators with fault current limiters," in 2011, pp. 1-8.

[142] C. Yuen and A. Oudalov, "The feasibility and profitability of ancillary services provision from multi-microgrids," in 2007, pp. 598-603. 
[143] M. A. Hossain, H. R. Pota, M. J. Hossain and F. Blaabjerg, "Evolution of microgrids with converter-interfaced generations: Challenges and opportunities," International Journal of Electrical Power \& Energy Systems, vol. 109, pp. 160-186, 2019.

[144] J. P. Lopes and N. J. Gil, "Exploiting Automated Demand Response, Generation and Storage Capabilities for Hierarchical Frequency Control in Islanded MultiMicrogrids," 2008.

[145] S. A. Arefifar, M. Ordonez and Y. A. I. Mohamed, "Energy management in multimicrogrid systems-Development and assessment," IEEE Trans. Power Syst., vol. 32, (2), pp. 910-922, 2016.

[146] F. H. Aghdam, J. Salehi and S. Ghaemi, "Contingency based energy management of multi-microgrid based distribution network," Sustainable Cities and Society, vol. 41, pp. 265-274, 2018.

[147] H. Wang and J. Huang, "Incentivizing energy trading for interconnected microgrids," IEEE Transactions on Smart Grid, vol. 9, (4), pp. 2647-2657, 2016.

[148] W. Chiu, H. Sun and H. V. Poor, "A multiobjective approach to multimicrogrid system design," IEEE Transactions on Smart Grid, vol. 6, (5), pp. 2263-2272, 2015.

[149] Y. Liu, Y. Li, H. B. Gooi, Y. Jian, H. Xin, X. Jiang and J. Pan, "Distributed robust energy management of a multimicrogrid system in the real-time energy market," IEEE Transactions on Sustainable Energy, vol. 10, (1), pp. 396-406, 2017.

[150] M. Xie, X. Ji, X. Hu, P. Cheng, Y. Du and M. Liu, "Autonomous optimized economic dispatch of active distribution system with multi-microgrids," Energy, vol. 153, pp. 479-489, 2018.

[151] B. Zhang, Q. Li, L. Wang and W. Feng, "Robust optimization for energy transactions in multi-microgrids under uncertainty," Appl. Energy, vol. 217, pp. 346-360, 2018.

[152] Y. Du, Z. Wang, G. Liu, X. Chen, H. Yuan, Y. Wei and F. Li, "A cooperative game approach for coordinating multi-microgrid operation within distribution systems," Appl. Energy, vol. 222, pp. 383-395, 2018.

[153] C. Zhang, J. Wu, Y. Zhou, M. Cheng and C. Long, "Peer-to-Peer energy trading in a Microgrid," Appl. Energy, vol. 220, pp. 1-12, 2018.

[154] A. G. Madureira, J. C. Pereira, N. J. Gil, J. P. Lopes, G. N. Korres and N. D. Hatziargyriou, "Advanced control and management functionalities for multi-microgrids," European Transactions on Electrical Power, vol. 21, (2), pp. 1159-1177, 2011. 
[155] D. Alert, "Cyber-attack against ukrainian critical infrastructure," Cybersecurity Infrastruct. Secur. Agency, Washington, DC, USA, Tech. Rep. ICS Alert (IR-ALERT-H16-056-01), 2016.

[156] T. Brennan, "Electricity markets and energy security: Friends or foes?" 2007.

[157] C. Weare, The California Electricity Crisis: Causes and Policy Options. Public Policy Instit. of CA, 2003.

[158] M. M. Esfahani and O. A. Mohammed, "Secure blockchain-based energy transaction framework in smart power systems," in 2018, pp. 260-264.

[159] G. Liang, S. R. Weller, F. Luo, J. Zhao and Z. Y. Dong, "Distributed blockchainbased data protection framework for modern power systems against cyber attacks," IEEE Transactions on Smart Grid, vol. 10, (3), pp. 3162-3173, 2018.

[160] Q. H. Dang, "Secure hash standard," 2015.

[161] M. M. Esfahani, A. Hariri and O. A. Mohammed, "A multiagent-based gametheoretic and optimization approach for market operation of multimicrogrid systems," IEEE Transactions on Industrial Informatics, vol. 15, (1), pp. 280-292, 2018.

[162] Y. Lai, "Hierarchical optimization: a satisfactory solution," Fuzzy Sets Syst., vol. 77, (3), pp. 321-335, 1996.

[163] M. Ruijgrok, "A single-item continuous double auction game," arXiv Preprint arXiv:1210. 5541, 2012.

[164] F. Meng, B. Chowdhury and M. Chamanamcha, "Three-phase optimal power flow for market-based control and optimization of distributed generations," IEEE Transactions on Smart Grid, vol. 9, (4), pp. 3691-3700, 2016.

[165] H. K. Nunna and D. Srinivasan, "Multiagent-based transactive energy framework for distribution systems with smart microgrids," IEEE Transactions on Industrial Informatics, vol. 13, (5), pp. 2241-2250, 2017.

[166] J. Martínez, J. Rodríguez-Molina, P. Castillejo and R. De Diego, "Middleware architectures for the smart grid: Survey and challenges in the foreseeable future," Energies, vol. 6, (7), pp. 3593-3621, 2013.

[167] S. Schneider and B. Farabaugh, "Is DDS for You?" A Whitepaper by Real-Time Innovations, pp. 1-5, 2009.

[168] G. Pardo-Castellote, "Omg data-distribution service: Architectural overview," in 2003, pp. 200-206. 
[169] D. C. Schmidt and H. van't Hag, "Addressing the challenges of mission-critical information management in next-generation net-centric pub/sub systems with opensplice dds," in 2008, pp. 1-8.

[170] O. I. Specification, "Object management group," Needham, MA, USA, vol. 2, (2), 2006.

[171] W. Kang, K. Kapitanova and S. H. Son, "RDDS: A real-time data distribution service for cyber-physical systems," IEEE Transactions on Industrial Informatics, vol. 8, (2), pp. 393-405, 2012.

[172] J. Durbin and R. W. Scharadin, "The modernization of the aegis fleet with open architecture," LOCKHEED MARTIN CORP CHERRY HILL NJ ADVANCED TECHNOLOGY LABS, 2011.

[173] R. Innovation, "RTI Routing Service for DDS (2010)," URL Http://Www. Rti. Com/Docs/RTI_Routing_Service. Pdf, .

[174] G. Ethernet, "RTI Connext® DDS Professional," .

[175] K. Beckman and J. Reininger, "Adaptation of the DDS security standard for resource-constrained sensor networks," in 2018, pp. 1-4.

[176] S. Ibba, A. Pinna, M. Seu and F. E. Pani, "CitySense: Blockchain-oriented smart cities," in 2017, pp. 1-5.

[177] J. Wang, Q. Wang, N. Zhou and Y. Chi, "A novel electricity transaction mode of microgrids based on blockchain and continuous double auction," Energies, vol. 10, (12), pp. 1971, 2017.

[178] K. Tweed, "Survey: 76\% of consumers don't trust their utility," Greentech Media, 2013.

[179] N. Wang, X. Zhou, X. Lu, Z. Guan, L. Wu, X. Du and M. Guizani, "When energy trading meets blockchain in electrical power system: The state of the art," Applied Sciences, vol. 9, (8), pp. 1561, 2019.

[180] T. Yang, Q. Guo, X. Tai, H. Sun, B. Zhang, W. Zhao and C. Lin, "Applying blockchain technology to decentralized operation in future energy internet," in 2017, pp. $1-5$.

[181] S. Sahoo, A. M. Fajge, R. Halder and A. Cortesi, "A Hierarchical and AbstractionBased Blockchain Model," Applied Sciences, vol. 9, (11), pp. 2343, 2019. 
[182] R. C. Dugan, W. H. Kersting, S. Carneiro, R. F. Arritt and T. E. McDermott, "Roadmap for the IEEE PES test feeders," in 2009, pp. 1-4.

[183] G. Carrasco, C. Silva, R. Peña and R. Cárdenas, "Control of a four-leg converter for the operation of a DFIG feeding stand-alone unbalanced loads," IEEE Trans. Ind. Electron., vol. 62, (7), pp. 4630-4640, 2014.

[184] A. O. Hariri, M. M. Esfahani and O. Mohammed, "A cognitive price-based approach for real-time management of en-route electric vehicles," in 2018, pp. 922-927.

[185] A. Konak, D. W. Coit and A. E. Smith, "Multi-objective optimization using genetic algorithms: A tutorial," Reliab. Eng. Syst. Saf., vol. 91, (9), pp. 992-1007, 2006.

[186] I. Y. Kim and O. de Weck L., "Adaptive weighted-sum method for bi-objective optimization: Pareto front generation," Structural and Multidisciplinary Optimization, vol. 29, (2), pp. 149-158, 2005.

[187] B. Sereeter, K. Vuik and C. Witteveen, "Newton power flow methods for unbalanced three-phase distribution networks," Energies, vol. 10, (10), pp. 1658, 2017.

[188] Y. Del Valle, G. K. Venayagamoorthy, S. Mohagheghi, J. Hernandez and R. G. Harley, "Particle swarm optimization: basic concepts, variants and applications in power systems," IEEE Transactions on Evolutionary Computation, vol. 12, (2), pp. 171-195, 2008.

[189] M. M. Esfahani, A. Sheikh and O. Mohammed, "Adaptive real-time congestion management in smart power systems using a real-time hybrid optimization algorithm," Electr. Power Syst. Res., vol. 150, pp. 118-128, 2017.

[190] M. M. Esfahani, H. F. Habib and O. A. Mohammed, "Optimal power routing scheme between and within interlinking converters in unbalanced hybrid AC-DC microgrids," IET Generation, Transmission \& Distribution, 2019.

[191] W. H. Kersting, "Radial distribution test feeders," IEEE Trans. Power Syst., vol. 6, (3), pp. 975-985, 1991.

[192] B. D. Muratori, "IEEE standard for calculating the current-temperature relationship of bare overhead conductors," IEEE Standard, pp. 738-2012, 2013.

[193] J. Kennedy and R. Eberhart, "Particle swarm optimization," in 1995, pp. 19421948.

[194] A. Al-Masri, M. Ab Kadir, H. Hizam and N. Mariun, "Simulation of an adaptive artificial neural network for power system security enhancement including control action," Applied Soft Computing, vol. 29, pp. 1-11, 2015. 
[195] M. M. Esfahani and O. Mohammed, "An intelligent protection scheme to deal with extreme fault currents in smart power systems," International Journal of Electrical Power \& Energy Systems, vol. 115, pp. 105434, 2020.

[196] T. A. Youssef, A. T. Elsayed and O. A. Mohammed, "Data distribution servicebased interoperability framework for smart grid testbed infrastructure," Energies, vol. 9, (3), pp. 150, 2016.

[197] P. C. I. PCI, "Data Security Standard," Update, vol. 64, pp. 2-0, 2010.

[198] M. Singh, V. Telukanta and K. S. Meera, "An Innovative Solution for Type Testing of Power Transformers," International Journal of Emerging Electric Power Systems, vol. 20, (1), 2019.

[199] K. Chen, C. Huang and J. He, "Fault detection, classification and location for transmission lines and distribution systems: a review on the methods," High Voltage, vol. 1, (1), pp. 25-33, 2016.

[200] T. S. Sidhu, D. S. Baltazar, R. M. Palomino and M. S. Sachdev, "A new approach for calculating zone-2 setting of distance relays and its use in an adaptive protection system," IEEE Trans. Power Del., vol. 19, (1), pp. 70-77, 2004.

[201] R. Ou, Y. Gun, Z. Yu, J. Wu, S. Chen and H. Zhen, "The Actual Operation of Transmission Line and Evaluation of the Reclosing Time of Reclosure," DEStech Transactions on Engineering and Technology Research, 2017.

[202] U. J. Minnaar, C. T. Gaunt and F. Nicolls, "Characterisation of power system events on South African transmission power lines," Electr. Power Syst. Res., vol. 88, pp. 25-32, 2012.

[203] W. Liss and P. Rowley, "Assessment of Natural Gas and Electric Distribution Service Reliability," Gas Technology Institute, 2018.

[204] M. Serra and F. Castro, "Using IEC 61850 for teleprotection," in 2007, pp. 21-24.

[205] C. Brunner, "IEC 61850 for power system communication," in 2008, pp. 1-6.

[206] Y. Damchi, M. Dolatabadi, H. R. Mashhadi and J. Sadeh, "MILP approach for optimal coordination of directional overcurrent relays in interconnected power systems," Electr. Power Syst. Res., vol. 158, pp. 267-274, 2018.

[207] L. Andersson, K. Brand, C. Brunner and W. Wimmer, "Reliability investigations for SA communication architectures based on IEC 61850," in 2005, pp. 1-7. 
VITA

\section{MOHAMMAD MAHMOUDIAN ESFAHANI}

1985 Born, Isfahan, Iran

2004-2009 B.Sc., Electrical Engineering, Shahid Rajaee University, Tehran, Iran.

2009-2012

M. Sc., Electrical Engineering, Isfahan University of Technology, Isfahan, Iran.

$2012-2016$

Power System Engineer, Esfahan Regional Electric Company, Isfahan, Iran.

2016-2020 Research Assistant, Florida International University, Miami, Florida, USA.

\section{SELECTED PUBLICATIONS AND PRESENTATIONS \\ Format: Patent $[\mathrm{P}-n]$, Journal $[\mathrm{J}-n]$, Conference $[\mathrm{C}-n]$,}

[P1]. M. M. Esfahani, Tarek Youssef, and Osama Mohammed. "Method and apparatuses for data integrity and security for communications in smart power systems", U.S. Patent 10,499,242 issued December 3, 2019.

[J-1] M. M. Esfahani, and Osama Mohammed. "An intelligent protection scheme to deal with extreme fault currents in smart power systems." International Journal of Electrical Power \& Energy Systems 115, 2020.

[J-2] M. M. Esfahani, Hany F Habib, Osama Mohammed, "Optimal Power Routing Scheme Between and Within Interlinking Converters in Unbalanced Hybrid AC-DC Microgrids “, IET Generation, Transmission \& Distribution, 2019.

[J-3] M. M. Esfahani, and Osama Mohammed. "Real-Time Distribution of En-route Electric Vehicles for Optimal Operation of Unbalanced Hybrid AC/DC Microgrids." eTransportation. 2019.

[J-4] M. M. Esfahani, A. Hariri and O. A. Mohammed, "A Multiagent-Based GameTheoretic and Optimization Approach for Market Operation of Multimicrogrid Systems," IEEE Transactions on Industrial Informatics, vol. 15, no. 1, pp. 280-292, Jan. 2019.

[J-5] M. M. Esfahani, Ahmed Sheikh and Osama Mohammed, "Adaptive Real-Time Congestion Management in Smart Power Systems Using a Real-Time Hybrid Optimization 
Algorithm ", Electric Power System Research (EPSR), Vol 150, Pages 118-128, 2017.

[C-1] M. M. Esfahani, Tarek Youssef, and Osama Mohammed, " Secure Data-Centric Communication Approach for IEC 61850 Routable GOOSE Messages ", Accepted for publication in IAS annual meeting 2020.

[C-2] M. M. Esfahani, Hany F Habib, Osama Mohammed, " Data-Centric Communication Approach for Energy Transactions in Multimicrogrid Systems", SoutheastCon 2020, Raleigh, NC, 2020.

[C-3] M. M. Esfahani, Osama Mohammed, "Secure Blockchain-based Energy Transaction Framework in Smart Power Systems," IEEE IECON 2018, Washington, DC, 2018.

[C-4] M. M. Esfahani, Hany F Habib, Osama Mohammed, "A Hierarchical Power Routing Scheme for Interlinking Converters in Unbalanced Hybrid AC-DC Microgrids," IEEE IECON 2018, Washington, DC, 2018.

[C-5] M. M. Esfahani, Abla Hariri and O. Mohammed, "Game-theory-based Real-Time Inter-Microgrid Market Design Using Hierarchical Optimization Algorithm" IEEE Power and Energy Society General Meeting, Portland Oregon, 2018.

[C-6] M. M. Esfahani and Osama Mohammed, "Loadability Improvement of Unbalanced Hybrid AC-DC Framework to Analyze Cyber and Physical Information Flow in Small DC Microgrids Using a Supervisory Control Scheme for Interlinking Converters" 2018 IEEE PES Innovative Smart Grid Technologies Asia, Singapore, 2018.

[C-7] M. M. Esfahani, H. F. Habib and O. A. Mohammed, "Microgrid Stability Improvement using a Fuzzy-Based PSS Design for Virtual Synchronous Generator," SoutheastCon 2018, St. Petersburg, FL, 2018.

[C-8] M. M. Esfahani, M. H. Cintuglu and O. A. Mohammed, "Optimal real-time congestion management in power markets based on particle swarm optimization," 2017 IEEE Power \& Energy Society General Meeting, Chicago, IL, 2017. 